\title{
A Comparison of Control Allocation Methods for the F-15 ACTIVE Research Aircraft Utilizing Real-Time Piloted Simulations
}

\author{
Kevin R. Scalera \\ Thesis submitted to the Faculty of the \\ Virginia Polytechnic Institute and State University \\ in partial fulfillment of the requirements for the degree of
}

Master of Science

in

Aerospace Engineering

Dr. Wayne Durham - chair

Dr. Mark Anderson

Dr. Frederick Lutze

July 1999

Blacksburg, Virginia

Keywords: Control Allocation, Aircraft Dynamics, ACTIVE, Reconfiguration Copyright 1999, Kevin R. Scalera 


\title{
A Comparison of Control Allocation Methods for the F-15 ACTIVE Research Aircraft Utilizing Real-Time Piloted Simulations
}

\author{
Kevin R. Scalera
}

\section{(ABSTRACT)}

A comparison of two control allocation methods is performed utilizing the F-15 ACTIVE research vehicle. The control allocator currently implemented on the aircraft is replaced in the simulation with a control allocator that accounts for both control effector positions and rates. Validation of the performance of this Moment Rate Allocation scheme through real-time piloted simulations is desired for an aircraft with a high fidelity control law and a larger control effector suite.

A more computationally efficient search algorithm that alleviates the timing concerns associated with the early work in Direct Allocation is presented. This new search algorithm, deemed the Bisecting, Edge-Search Algorithm, utilizes concepts derived from pure geometry to efficiently determine the intersection of a line with a convex faceted surface.

Control restoring methods, designed to drive control effectors towards a "desired" configuration with the control power that remains after the satisfaction of the desired moments, are discussed. Minimum-sideforce restoring is presented. In addition, the concept of variable step size restoring algorithms is introduced and shown to yield the best tradeoff between restoring convergence speed and control chatter reduction.

Representative maneuvers are flown to evaluate the control allocator's ability to perform during realistic tasks. An investigation is performed into the capability of the control allocators to reconfigure the control effectors in the event of an identified control failure. More specifically, once the control allocator has been forced to reconfigure the controls, an investigation is undertaken into possible performance degradation to determine whether or not the aircraft will still demonstrate acceptable flying qualities.

A direct comparison of the performance of each of the two control allocators in a reduced global position limits configuration is investigated. Due to the highly redundant control effector suite of the F-15 ACTIVE, the aircraft, utilizing Moment Rate Allocation, still exhibits satisfactory performance in this configuration. The ability of Moment Rate Allocation to utilize the full moment generating capabilities of a suite of controls is demonstrated. 


\section{Acknowledgments}

I would like to extend my most sincere gratitude to my parents for their support and encouragement over the years. They have inspired me to make the most of myself and to live each day to the fullest. It is because of their guidance and direction that I find myself continously striving towards the top with aspirations of overcoming any obstacle along the way. To my brothers Steve and Jon between whom I have been fortunate enough to have grown up. With Steve's endless effort to lead the way, blazing new trails and setting benchmarks at each step, and with Jon's relentless desire to never stay in the shadow of Steve and I, it has thus far been an exhausting yet rewarding quest through academia and life.

I must thank my advisor Dr. Wayne Durham for his persistence and patience with me. He took me under his wing and pushed me to discover new things and really question and ponder what had already been found. He always challenged me to try to figure out the solution to a problem on my own before seeing how somebody else solved it, and for this and his ceaseless tutelage I am deeply indebted to him. To the remaining members of my committee, Dr. Fred Lutze and Dr. Mark Anderson, thank you for helping to guide me through my education and for sharing your seemingly endless knowledge with me.

I would also like to acknowledge the help and support that Keith Balderson and his group at Manned Flight Simulator have given in support of making the Flight Simulation Lab the facility it is today. To Jim Buckley and John Bolling at Boeing Phantom Works, thank you for offering debugging hints as well as other necessary data crucial to the implementation of the F-15 ACTIVE model and the completion of my research.

I must thank my friends that have helped to make my stay at Virginia Tech so enjoyable. To Drew Robbins, Greg Stagg, Bill Oetjens, Dan Lluch, Roger Beck, Mike Phillips, Michelle Glaze, Jeff Leedy, Josh Durham, Abhishek Kumar and Mark Nelson, thank you for creating a work environment in the Simlab that was second to none. To the rest of the Aerospace crew, 
especially Cindy Whitfield, John Prebola, Alex Remington, Samantha Magill, Matt Long, Troy Jones and Mike Goody, thanks for two years of nonstop fun, innumerous memories and for sharing so much time and so many experiences with me outside of work. Finally, I would like to thank my friends from back home who have offered me support and encouragement from hundreds of miles away and kept true to their promise to never lose touch.

Financial support for this research project was provided by the Naval Air Warfare Center, Aircraft Division under Contract N00178-98-D-3017. The views and conclusions contained herein are those of the author and should not be interpreted as necessarily representing the official policies or endorsements, either expressed or implied, of Naval Air Warfare Center or any other agency of the U.S. Government. 


\section{Contents}

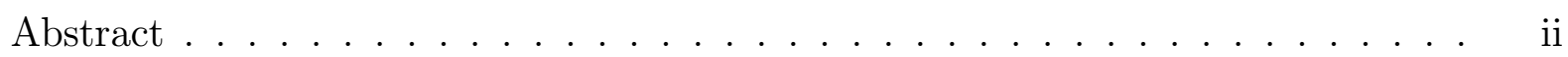

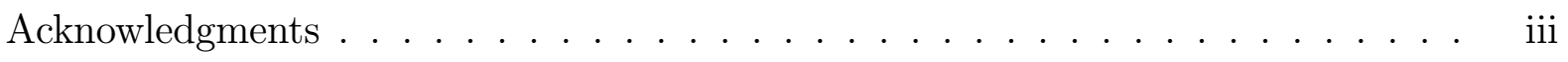

Table of Contents .......................

List of Figures . . . . . . . . . . . . . . . . . ix

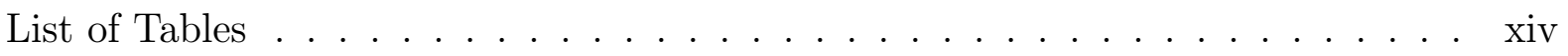

Nomenclature . . . . . . . . . . . . . . . . . . xv

1 Introduction 1

1.1 Background . . . . . . . . . . . . . . . . . 1

1.2 Research Objectives . . . . . . . . . . . . . . . . . . . 3

1.3 Suggestions to the Reader $\ldots \ldots \ldots \ldots \ldots$

$\begin{array}{lrlr}2 & \text { F-15 ACTIVE } & 6\end{array}$

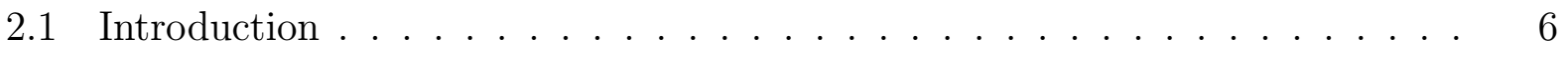

2.2 ACTIVE Objectives $\ldots \ldots \ldots \ldots \ldots \ldots \ldots \ldots \ldots \ldots \ldots \ldots \ldots \ldots$

2.3 Hardware . . . . . . . . . . . . . . . . . . . 7

2.4 Control Law . . . . . . . . . . . . . . . . . . 8

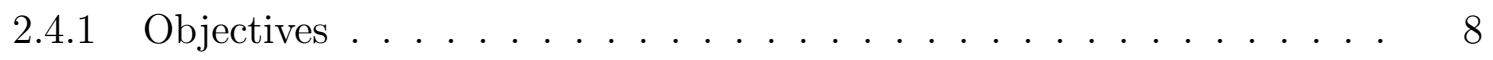

2.4 .2 Dynamic Inversion Format . . . . . . . . . . . . . . . . . . . 9 
2.4.3 Determing Desired Moments . . . . . . . . . . . . . 12

2.5 Simulation Code and Environment . . . . . . . . . . . . . 14

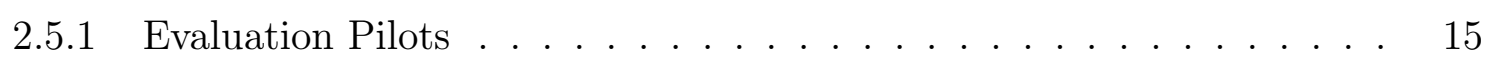

2.5.2 Handling Qualities Evaluation Using Real-Time Simulation . . . . . . 16

2.6 Code Verification . . . . . . . . . . . . . . . . . . 17

3 Control Allocation Theory $\quad 19$

3.1 Allocation Methods . . . . . . . . . . . . . . . . 19

3.2 Frame-wise Control Allocation . . . . . . . . . . . . . . . . . 20

3.3 Direct Allocation Solution . . . . . . . . . . . . . . . . 22

4 Bisecting, Edge-Searching Algorithm 24

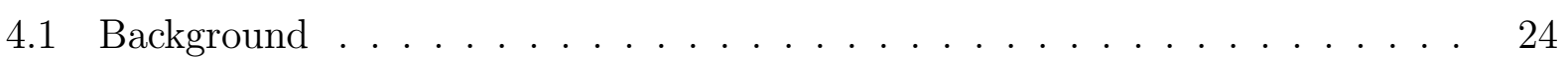

4.2 Two-Dimensional Problem . . . . . . . . . . . . . . . . 25

4.3 Three-Dimensional Problem . . . . . . . . . . . . . . . . . 31

4.4 Timing Analysis . . . . . . . . . . . . . . . . . . 36

4.4.1 Allocation Methods Investigated . . . . . . . . . . . . 36

4.4.2 Required Floating Point Operations . . . . . . . . . . . . . . 37

4.4 .3 Error Analysis . . . . . . . . . . . . . . . . . . 39

4.4 Timing Conclusions . . . . . . . . . . . . . . . 41

5 Restoring Methods $\quad 43$

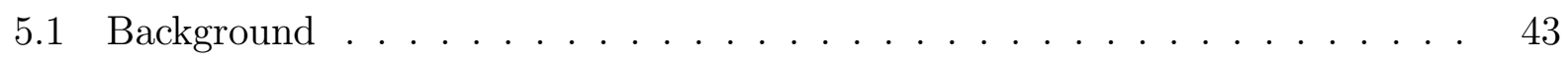

5.2 Restoring to a Known Solution . . . . . . . . . . . . . . . 45

5.2.1 Minimum-Norm Restoring: Known Solution . . . . . . . . . . . . 46

5.3 Restoring to an Unknown Solution . . . . . . . . . . . . . . 47 
5.4 Minimum-Sideforce Restoring . . . . . . . . . . . . . . . . . . . 48

5.4.1 Variable Step Size Restoring . . . . . . . . . . . . . . . . . . . 49

6 Representative Maneuvers $\quad 52$

6.1 Canned Input: Lateral Stick Doublet . . . . . . . . . . . . . . . . . 52

6.2 Air Combat Maneuvering: High Yo-Yo . . . . . . . . . . . . . . . 61

6.2 .1 High Yo-Yo: Description . . . . . . . . . . . . . . . . . . . . 61

6.2 .2 High Yo-Yo: Simulator Implementation . . . . . . . . . . . . . 63

6.2.3 High Yo-Yo: Task Desired Performance . . . . . . . . . . . . . . . . 64

6.2 .4 High Yo-Yo: Results . . . . . . . . . . . . . . . . . . . . . 65

6.2 .5 High Yo-Yo: Minimum-Sideforce Restoring . . . . . . . . . . . . 72

6.3 Terrain Following . . . . . . . . . . . . . . . . . 73

6.3.1 Terrain Following: Description . . . . . . . . . . . . 73

6.3.2 Terrain Following: Task Desired Performance . . . . . . . . . . 74

6.3 .3 Terrain Following: Results . . . . . . . . . . . . . 76

7 Control Reconfiguration $\quad 83$

7.1 Background . . . . . . . . . . . . . . . . . 83

7.2 Implementation . . . . . . . . . . . . . . . 84

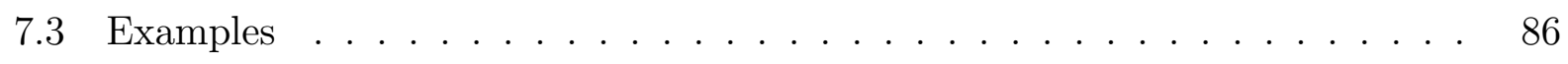

7.3.1 Lateral Stick Doublet: Aileron Failure _. . . . . . . . . . 87

7.3 .2 High Yo-Yo: Aileron Failure . . . . . . . . . . . . . . . 88

7.3.3 High Yo-Yo: Double Horizontal Tail Failure . . . . . . . . . . . 96

7.3.4 Terrain Following: Double Horizontal Tail Failure . . . . . . . . . 103

7.4 Viability of MRA for Control Reconfiguration . . . . . . . . . . . 111 
8 Performance Comparison $\quad 113$

8.1 Euclidean Norm Comparison . . . . . . . . . . . . . . . . . . . . 113

8.2 Reduced Global Position Limits . . . . . . . . . . . . . . . . 116

8.2.1 Lateral Stick Doublet: Reduced Controls Configuration . . . . . . . . 119

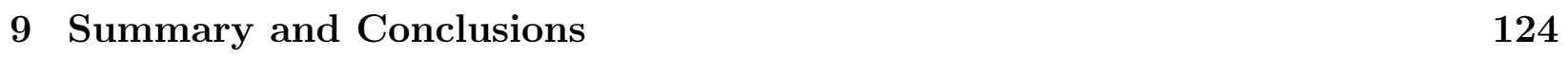

$\begin{array}{lr}\text { Bibliography } & 128\end{array}$

$\begin{array}{ll}\text { A F-15 ACTIVE Specifications } & 131\end{array}$

$\begin{array}{ll}\text { B Control Effectiveness Extraction Code } & 137\end{array}$

B.1 Subroutine Bgetter.c . . . . . . . . . . . . . . . . . . 137

B.1.1 Description . . . . . . . . . . . . . . . . 137

B.1.2 Code . . . . . . . . . . . . . . . . . . . . . . . 138

B.2 Subroutine Jacob.f . . . . . . . . . . . . . . . . . . . . . . 151

B.2.1 Description . . . . . . . . . . . . . . . . . 151

B.2.2 Code . . . . . . . . . . . . . . . . . . . . . . 152

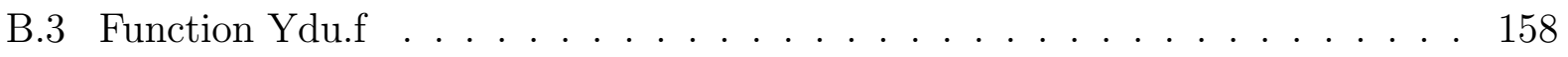

B.3.1 Description . . . . . . . . . . . . . . . . . 158

B.3.2 Code . . . . . . . . . . . . . . . . . . . . . . 158

$\begin{array}{lr}\text { Vita } & 166\end{array}$ 


\section{List of Figures}

$2.1 \quad$ F-15 ACTIVE hardware components. . . . . . . . . . . . . . 7

2.2 Affine control effectiveness data $\left(\partial C_{l} / \partial \delta_{A I L L}\right)$ as a function of Mach number for the F-15 ACTIVE at $10,000 \mathrm{ft} \ldots \ldots \ldots \ldots \ldots$

2.3 General format of control law - control allocator framework. . . . . . . . . . 12

2.4 Standard stability-axis system. . . . . . . . . . . . . . . . . . . . . . . 13

4.1 Two-dimensional AMS for the F-15 ACTIVE. Small dots represent the vertices of the AMS. $x$ and $y$ axes correspond to non-dimensional pitching and yawing moment coefficients, respectively. . . . . . . . . . . . . . . 26

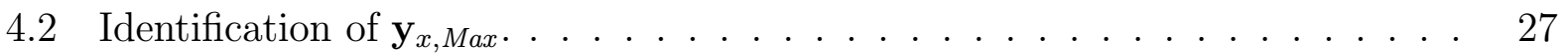

4.3 Rotation by $\phi$ to determine $\mathbf{y}_{x, \text { Max }}^{\prime} \ldots \ldots \ldots \ldots \ldots$

4.4 Rotated AMS with $\mathbf{m}_{\mathbf{d}}$ aligned with $x$-axis. Solution edge determined by finding a change in sign of the $y$-component of two consecutive vertices. . . . 30

4.5 Three-Dimensional AMS for the F-15 ACTIVE at $400 \mathrm{knots}$ and 10,000 $\mathrm{ft}$ with twelve controls. Arbitrary AMS orientation depicted. . . . . . . . . . 32

4.6 Three-Dimensional AMS for the F-15 ACTIVE at $400 \mathrm{knots}$ and 10,000 $\mathrm{ft}$ with twelve controls. AMS oriented such that $\ell$ is aligned with the positive $x$-axis and viewed from the vantage point of the positive $z$-axis. . . . .

4.7 Three-Dimensional AMS for the F-15 ACTIVE at 400 knots and 10,000 ft with twelve controls. AMS oriented such that $\ell$ is aligned with the positive $x$-axis and viewed from the vantage point of the positive $z$-axis. AMS has been rotated about the $x$-axis to place the intersection point on the limb. . . 
4.8 Required number of floating point operations for brute-force facet-search. . . 37

4.9 Required number of floating point operations, other methods. . . . . . . 38

4.10 Number of errors using Edge-Searching Algorithm. . . . . . . . . . . . . 40

4.11 Percentage error using Edge-Searching Algorithm. . . . . . . . . . . . . 41

5.1 Roll rate and sideforce generated during a 3 inch lateral stick doublet for the F-15 ACTIVE at 400 knots and 10,000 ft. . . . . . . . . . . . . . 48

5.2 Time history of sideforce, left canard deflection and restoring step size during a 3 inch lateral stick doublet for the F-15 ACTIVE at 400 knots and 10,000 ft. 51

6.1 Horizontal tail and aileron responses to 4 inch lateral stick doublet at 400 knots and 10,000 ft. . . . . . . . . . . . . . . 56

6.2 Rudder and canard responses to 4 inch lateral stick doublet at 400 knots and $10,000 \mathrm{ft}$. . . . . . . . . . . . . . . . . . 57

6.3 Pitch and yaw vectoring responses to 4 inch lateral stick doublet at $400 \mathrm{knots}$ and $10,000 \mathrm{ft} \ldots \ldots \ldots \ldots \ldots \ldots$

6.4 Roll, pitch and yaw rate responses to 4 inch lateral stick doublet at $400 \mathrm{knots}$ and $10,000 \mathrm{ft} . \ldots \ldots \ldots \ldots$. . . . . . . . . . . . . . . 59

6.5 Angle-of-attack, sideslip angle, normal and lateral Acceleration responses to 4 inch lateral stick doublet at 400 knots and $10,000 \mathrm{ft}$. . . . . . . . . . . 60

6.6 Time history of High Yo-Yo maneuver with attacker and bogey trajectories shown in blue and red, respectively. Dotted green lines drawn between the two aircraft cg's at 2.5 second intervals. . . . . . . . . . . . . . . . . . 62

6.7 Time history of attacker and bogey during High Yo-Yo maneuver. Bearing and range shown throughout the maneuver. Dashed red and green lines indicate adequate and desired ranges, respectively. . . . . . . . . . . . . 69

6.8 Time history of control effector positions during High Yo-Yo maneuver with no failures and no restoring. . . . . . . . . . . . . . 70 
6.9 Time history of aircraft states during High Yo-Yo maneuver with no failures and no restoring. . . . . . . . . . . . . . . . . . 71

6.10 Sideforce on Aircraft during High Yo-Yo maneuver with and without minsideforce restoring. . . . . . . . . . . . . . . . . . 72

6.11 Desired trajectory during terrain following task. Pilot must keep red buildings to his right and green building to his left while maintaining an altitude of under $300 \mathrm{ft}$ during the maneuver. . . . . . . . . . . . . . . 74

6.12 Contour of left trailing edge flap pitch effectiveness as a function of angle-ofattack and control deflection. F-15 ACTIVE at Mach 0.1 and $100 \mathrm{ft}$. . . . 77

6.13 Trajectory and altitude during terrain following task with no failures. . . . . 80

6.14 Control effector deflections during terrain following task with no failures. . . 81

6.15 Aircraft states during terrain following task with no failures. . . . . . . . . 82

7.1 Lateral stick doublet with minimum-norm restoring with/without a right aileron failure. 4, 3 and 2 inch lateral stick doublets shown for the F-15 ACTIVE at 400 knots and $10,000 \mathrm{ft} \ldots \ldots \ldots \ldots . \ldots . \ldots 8$

7.2 Right aileron highlighted in gray to show contribution to AMS. F-15 ACTIVE at 400 knots and $10,000 \mathrm{ft} . \ldots \ldots \ldots \ldots$

7.3 AMS after right aileron hard-over to +20 degrees. F-15 ACTIVE at 400 knots

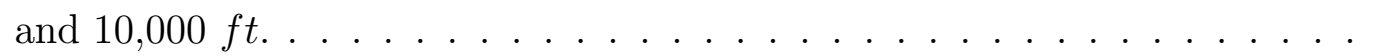

7.4 Time history of attacker and bogey during High Yo-Yo maneuver with right aileron failure. Bearing and range shown throughout the maneuver. Dashed red and green lines indicate adequate and desired ranges, respectively. . . . .

7.5 Time history of control effector positions during High Yo-Yo maneuver with right aileron failure. Minimum-norm restoring implemented. . . . . . . . . . 94

7.6 Time history of aircraft states during High Yo-Yo maneuver with right aileron failure. Minimum-norm restoring implemented. . . . . . . . . . . . 95

7.7 Facets associated with left and right horizontal tails highlighted in gray to show contribution to AMS. F-15 ACTIVE at 400 knots and 10,000 ft. . . . 96 
7.8 AMS after left and right horizontal tail jammed at 0 degrees. F-15 ACTIVE at 400 knots and $10,000 \mathrm{ft}$. . . . . . . . . . . . . . 97

7.9 Time history of attacker and bogey during High Yo-Yo maneuver with double horizontal tail failure. Bearing and range shown throughout the maneuver. Dashed red and green lines indicate adequate and desired ranges, respectively. 100

7.10 Time history of control effector positions during High Yo-Yo maneuver with double horizontal tail failure. . . . . . . . . . . . . . . . . . . . . . 101

7.11 Time history of aircraft states during High Yo-Yo maneuver with double horizontal tail failure. . . . . . . . . . . . . . . . . . . . . 102

7.12 Left and right horizontal tails highlighted in gray to show contribution to AMS. F-15 ACTIVE at 200 knots and 200 ft, flaps-down configuration. . . . 103

7.13 AMS after left and right horizontal tails jammed at 0 degrees. F-15 ACTIVE at 200 knots and $200 \mathrm{ft}$, flaps-down configuration. . . . . . . . . . . 104

7.14 Trajectory and altitude during terrain following task with double horizontal tail failure. . . . . . . . . . . . . . . . . . . . 105

7.15 Control effector deflections during terrain following task with double horizontal tail failure. . . . . . . . . . . . . . . . . . . 109

7.16 Aircraft states during terrain following task with double horizontal tail failure. 110

8.1 Euclidean norm of control effector positions as well as roll rate achieved during a 4 inch lateral stick doublet at 400 knots and 10,000 ft. . . . . . . . . . 114

8.2 Euclidean norm of control effector positions for the ACTIVE mixer and MRA with minimum-norm restoring during the High Yo-Yo maneuver. . . . . . . . 116

8.3 AMS before and after reduction in control effector position limits by a factor of two. F-15 ACTIVE at 400 knots and 10,000 ft. . . . . . . . . . 118

8.4 Roll rates achieved in reduced control effector position limits configuration for 4, 3 and 2 inch lateral stick doublets. Original mixer with and without control reduction as well as MRA mixer with reduced controls evaluated. . . . . . . 121 
8.5 Horizontal tails, ailerons and canard responses to 3 inch lateral stick doublet with reduced control limits. Yellow hatching indicates the saturation of a control surface. . . . . . . . . . . . . . . . . . . . . . . . 122

8.6 Rudder, pitch vectoring and yaw vectoring responses to 3 inch lateral stick doublet with reduced control limits. . . . . . . . . . . . . . . . . 123 


\section{List of Tables}

2.1 Control surface limits . . . . . . . . . . . . . . . . . . . . 8

3.1 Control effectiveness breakpoints. . . . . . . . . . . . . . . . . . 21

6.1 Summary of desired and adequate performance in the High Yo-Yo maneuver. 65

7.1 Minimum/maximum attainable moments for the F-15 ACTIVE with 12 controls at 400 knots and $10,000 \mathrm{ft} . \ldots \ldots \ldots \ldots$

7.2 Minimum/maximum attainable moments for the F-15 ACTIVE with 12 controls at 400 knots and 10,000 ft. Right aileron jammed at the +20 degree stop. . . . . . . . . . . . . . . . . . . . . . 91

7.3 AMS volume with and without failed controls. . . . . . . . . . . 96

7.4 Minimum/maximum attainable moments for the F-15 ACTIVE with 12 controls at 400 knots and 10,000 ft. Left and right horizontal tail jammed at 0 degrees. . . . . . . . . . . . . . . . . . . 97

7.5 Minimum/maximum attainable moments for the F-15 ACTIVE with 12 controls at 200 knots and $200 \mathrm{ft}$, flaps-down configuration. Left and right horizontal tails jammed at 0 degrees. . . . . . . . . . . . . . . . . . . 104

8.1 Reduced control surface limits. . . . . . . . . . . . . . . . . . . . . . . 117

8.2 Minimum/maximum attainable moments for the F-15 ACTIVE with 12 controls in reduced control deflection configuration at $400 \mathrm{knots}$ and 10,000 ft. . 118 


\section{Nomenclature}

Acronyms:

ACTIVE Advanced Control Technology for Integrated VEhicles

BESA Bisecting, Edge-Search Algorithm

CASTLE Control Analysis and Simulation Test Loop Environment

CGI Cascaded Generalized Inverse

HARV High Angle-of-attack Research Vehicle

MRA Moment Rate Allocation

S/MTD Short Takeoff and Landing / Maneuver Technology Demonstrator

Control Allocation Variables:

$\mathbf{B}^{\mathbf{3} \times \mathbf{m}-\mathbf{r}} \quad$ Control effectiveness matrix after $r$ failures

$\mathbf{B}_{\text {Aug }}^{4 \times \mathbf{m}-\mathbf{r}} \quad$ Augmented control effectiveness matrix after $r$ failures

B global Global control effectiveness matrix

$\mathbf{B}_{\mathbf{k}} \quad$ Control effectiveness matrix for the $k^{\text {th }}$ frame

$C_{s} \quad$ Scale factor used to ensure the restored control solution lies within $\omega$

$C_{m_{i}} \quad i^{\text {th }}$ non-dimensional moment coefficient

$\delta_{\text {STICK }} \quad$ Control inceptor deflections (deg)

$\Delta t \quad$ Computational frame length (sec) 
$\ell \quad$ Half-line in the direction of the desired moment/objective

$\Delta \mathbf{m}_{\mathbf{d}} \quad$ Vector of desired delta moments (ft-lbs)

$\Delta \mathbf{m}_{\mathbf{d}_{\mathbf{k}}} \quad$ Vector of desired delta moments for the $k^{\text {th }}$ frame (ft-lbs)

$\mathbf{m}_{\mathbf{d}} \quad$ Vector of desired moments (ft-lbs)

$m \quad$ Number of control effectors

$n \quad$ Number of objectives

$N_{\text {Bisections }}$ Maximum number of bisections used in searching algorithm

$\Omega \quad$ Subset of admissible controls

$\omega \quad$ More restrictive of global position limits and rate limits on control effectors

$\partial(\Phi) \quad$ Convex hull of the AMS

$\Phi \quad$ Attainable moment subset - AMS

$\phi_{j} \quad$ Rotation angle used in 2-d problem to drive $j^{\text {th }}$ entry of $r_{1}^{\prime}$ to zero (deg)

$p \quad$ Intersection point of $l$ on the convex hull of the AMS

$r \quad$ Number of failed controls

$T_{\theta} \quad$ Rotation matrix to rotate the AMS in 3 -d about the $x$-axis

$T_{x} \quad$ Transformation matrix to align the half-line $l$ with the $x$-axis

$\Delta \mathbf{u}_{k}^{\perp} \quad$ Delta vector of controls in the null space of $\mathbf{B}$ for the $\mathrm{k}^{\text {th }}$ iteration (deg)

$\Delta \mathbf{u}_{\text {rest }, k} \quad$ Restoring delta to the controls for the $\mathrm{k}^{\text {th }}$ iteration (deg)

$\Delta \mathbf{u}_{\text {rest }} \quad$ Restoring delta to the controls (deg)

$\mathbf{u}_{\mathbf{x}, \text { Max }}^{\prime} \quad$ Controls in $\Omega$ related to max $x$-comp. of $\Phi$ after rotation by $\phi_{j}(\mathrm{deg})$

$\mathbf{u}^{\perp} \quad$ Vector of controls in the null space of $\mathbf{B}(\mathrm{deg})$

$\mathbf{u}_{k}^{\perp} \quad$ Vector of controls in the null space of $\mathbf{B}$ for the $\mathrm{k}^{\text {th }}$ iteration (deg) 


$\begin{array}{ll}\mathbf{u}_{\mathbf{x}, \text { Max }} & \text { Controls in } \Omega \text { corresponding to max. } x \text {-comp. of } \Phi(\mathrm{deg}) \\ \dot{u}_{\text {max }_{i}} & \text { Maximum rate of the } i^{\text {th }} \text { control in the positive direction }(\mathrm{deg} / \mathrm{sec}) \\ \dot{u}_{\text {min }_{i}} & \text { Maximum rate of the } i^{\text {th }} \text { control in the negative direction (deg/sec) } \\ u_{\text {max }_{i}} & \text { Positive position limit for the } i^{\text {th }} \text { control effector }(\mathrm{deg}) \\ u_{\text {min }_{i}} & \text { Negative position limit for the } i^{\text {th }} \text { control effector }(\mathrm{deg}) \\ \Delta \mathbf{y}_{\mathbf{d}} & \text { Vector of desired delta objectives } \\ \mathbf{y}_{\mathbf{d}} & \text { Vector of desired objectives } \\ \mathbf{y}_{\mathbf{x}, \text { Max }}^{\prime} & \text { Vertex defining maximum } x \text {-component of } \Phi \text { after rotation by } \phi_{j} \\ \mathbf{y}_{\mathbf{x}, \text { Max }} & \text { Vertex defining maximum } x \text {-component of } \Phi\end{array}$

Aircraft Variables:

$\begin{array}{ll}\alpha & \text { Angle-of-attack (deg) } \\ b & \text { Aircraft span (ft) } \\ \bar{c} & \text { Aircraft mean geometric chord }(\mathrm{ft}) \\ I_{x x}, I_{y y}, I_{z z} & \text { Moments of inertia about the } \mathrm{x}, \mathrm{y}, \text { and } \mathrm{z} \text { axis }\left(\mathrm{slug}^{-\mathrm{ft}^{2}}\right) \\ n_{z} & \text { Aircraft load factor }(\mathrm{g}) \\ \dot{P}_{c m d} & \text { Commanded body-axis roll acceleration }\left(\mathrm{deg} / \mathrm{sec}^{2}\right) \\ P & \text { Body-axis roll rate }(\mathrm{deg} / \mathrm{sec}) \\ \bar{q} & \text { Dynamic pressure }\left(\mathrm{slug} / \mathrm{ft}{ }^{2}\right) \\ \dot{Q}_{c m d} & \text { Commanded body-axis pitch acceleration }\left(\mathrm{deg} / \mathrm{sec}^{2}\right) \\ Q & \text { Body-axis pitch rate }(\mathrm{deg} / \mathrm{sec}) \\ \dot{R}_{c m d} & \text { Commanded body-axis yaw acceleration }\left(\mathrm{deg} / \mathrm{sec}^{2}\right) \\ R & \text { Body-axis yaw rate }(\mathrm{deg} / \mathrm{sec})\end{array}$


Aircraft wing area $\left(\mathrm{ft}^{2}\right)$

$X_{S}, Y_{S}, Z_{S} \quad$ Stability-axis system coordinates

$X_{B}, Y_{B}, Z_{B}$ Body-axis system coordinates

Control Effector Abbreviations:

DAILL Left aileron position (deg)

DAILR Right aileron position (deg)

DCANL Left canard position (deg)

DCANR Right canard position (deg)

DHTL Left horizontal tail position (deg)

DHTR Right horizontal tail position (deg)

DPVL Left pitch thrust vectoring position (deg)

DPVR Right pitch thrust vectoring position (deg)

DRUDL Left rudder position (deg)

DRUDR Right rudder position (deg)

DTEFL Left trailing edge flap position (deg)

DTEFR Right trailing edge flap position (deg)

DYVL Left yaw thrust vectoring position (deg)

DYVR Right yaw thrust vectoring position (deg) 


\section{Chapter 1}

\section{Introduction}

\section{$1.1 \quad$ Background}

Aircraft flight controls are classically designed utilizing one controller or effector for each rotational degree of freedom. Essentially, the elevator is used to generate a pitching moment, the ailerons are used differentially to produce a rolling moment and the rudder controls the yawing moment of the aircraft. The control allocation problem is defined as the determination of the control effector positions that generate a given set of desired or commanded moments. In a classical airplane, with three desired moments and three independent control effectors to generate these moments, a unique solution is defined. However, in modern tactical aircraft, control effectors are no longer limited to the classical three. Aircraft can generate moments utilizing non-traditional control surfaces, such as canards, thrust vectoring, differential flaps, etc. In addition, opposing control surfaces have become independent of one another. For example, the left and right horizontal tails can be used differentially to create not only a pitching moment, but also yawing and rolling moments.

As aircraft performance demands continue to grow, the number of control surfaces on advanced tactical aircraft keeps rising. In fact, the aircraft that is the focus of this work, the F-15 ACTIVE (Advanced Control Technology for Integrated VEhicles), has 14 independent control effectors. (horizontal tails, ailerons, canards, pitch thrust vectoring, yaw thrust vectoring, rudders and flaps, each left and right) However, in order to maintain some consistency with the aircraft's original control allocation scheme, the work contained herein will assume that when the flaps are up, they will not be used for maneuvering. Therefore, in the 
flaps-up configuration the flaps will be excluded from the allocation problem and the number of control effectors will be reduced to 12. In the powered approach mode, (ie. flaps-down configuration), the ailerons and flaps are drooped to 20 degrees and utilized differentially for increased maneuvering capability.

With the increase in number of control effectors, the problem of allocating these controls to achieve the desired moments becomes far more complex. For systems with multiply redundant control effectors, there will, in general, exist an infinite number of control combinations that can attain the desired moments. Not all of these control effector combinations are attainable due to both physical geometry and aerodynamic constraints on control surface ranges of motion and rate capabilities. Thus, the solution to the control allocation problem must acknowledge these constraints and only command admissible control combinations.

Recent work by Durham and Leedy [1] investigated the real-time implementation of Direct Allocation on a computational frame-wise basis. The author understands that any of the numerous existing control allocation methods can be performed on a frame-wise basis. However, for the remainder of this work, Moment Rate Allocation (MRA) will refer to Direct Allocation computed on a frame-wise basis. Any exception to this convention will be otherwise explictly stated. Moment Rate Allocation, which accounts for position and rate limits, was previously simulated in batch mode by Bolling. [2] Durham and Leedy's work also investigated the utilization of restoring methods in an attempt to drive the control effectors back to a desired configuration upon completion of a maneuver. Their work demonstrated the viability of incorporating MRA in a real-time piloted simulation. However, further exercise of MRA as applied to an aircraft with a larger control effector suite and higher complexity control law is necessary to confirm the ability of MRA to perform in the environment that is associated with tactical aircraft of today and tomorrow.

Computational complexity of control allocation algorithms is of high importance with respect to implementation on an actual aircraft. The desire to limit the time involved with control allocation calculations prompted the search for a faster more efficient method of allocation. This paper introduces the Bisecting, Edge-Searching Algorithm, a variation of Moment Rate Allocation. [3] This algorithm replaces the computationally intensive facet-searching portion of MRA while still achieving nearly the same optimal solutions as the original MRA algorithms. [4] It will be shown that the computational complexity of this algorithm increases linearly with the number of control effectors, $m$, rather than quadratically as in the original Direct Allocation work. The theory behind the method is presented for the reader in 
Chapter 3. All work contained herein utilizes the Bisecting, Edge-Searching Algorithm in the solution of the near-optimal control allocation problem.

\section{$1.2 \quad$ Research Objectives}

The objective of this paper is to investigate the implementation of two control allocation schemes on the F-15 ACTIVE testbed. Real-time pilot-in-the-loop simulation of these control mixers will be used to evaluate their performance. A comparison of the current F-15 ACTIVE control mixer developed by Boeing Phantom Works and the Moment Rate Allocation mixer developed by Durham and Bordignon is performed [5].

Durham and Leedy's recent research on real-time implementation of Moment Rate Allocation which focused on simulations utilizing the F-18 HARV (High Angle-of-Attack Research Vehicle) was limited to the allocation of five independent control surfaces. In addition, the aircraft model they implemented in their real-time simulation contained only a low-level control law that translated stick position directly into desired moments. Unfortunately, this control law did not take into account actual aircraft handling qualities specifications. It is the intent of this research to further expand upon the knowledge gained by Durham and Leedy and investigate the viability of utilizing MRA real-time on an aircraft with a larger number of multiply redundant control effectors and an established control law that acknowledges handling qualities requirements for tactical aircraft.

Leedy and Durham performed a low-level investigation into the computational time involved with control allocation scheme. [1] Their results showed that the original brute force method of searching the geometric attainable moment subset for the solution was very computationally intensive. The research presented in this paper utilizes a more computationally efficient search algorithm that alleviates the timing concerns associated with the early work in Direct Allocation. This new search algorithm, deemed the Bisecting, Edge-Search Algorithm (BESA), utilizes concepts derived from pure geometry to efficiently determine the intersection of a line with a convex faceted surface. The concern that the control allocation calculations could not be completed in the time allotted to the flight control computer to perform all the necessary computations is shown to no longer be of great importance. In this regard, real-time implementation of Moment Rate Allocation is shown to be viable.

Control restoring methods have existed in the literature for years. These methods are de- 
signed to drive control effectors towards a "desired" configuration with the control power that remains after the satisfaction of the desired moments. This paper introduces a new restoring method; minimum-sideforce restoring. In addition, the concept of variable step size restoring algorithms is introduced. These algorithms offer the best tradeoff between restoring convergence speed and control chatter reduction.

A further objective of this paper is to look into the capability of these control allocators to perform control reconfiguration in the event of an identified failure. More specifically, once the control allocator has been forced to reconfigure the controls, an investigation into possible performance degradation is undertaken to determine whether or not the aircraft will still demonstrate acceptable flying qualities. The control reconfiguration analysis will only be performed with the MRA mixer since the original control mixer on board the ACTIVE does not accommodate failure reconfiguration.

Example maneuvers are flown with the aircraft in a control failure configuration to examine the potential degradation in aircraft performance due to the failures. The pilot was blind to the configuration of the aircraft being flown during each run in order to eliminate the possibility of him tailoring his piloting technique to accommodate for a particular restoring method or control failure. Several control failure combinations are investigated in this research. An aileron hard-over is included to examine the potential undesired roll and yaw generation due to a non-symmetric control failure configuration. A loss of the left and right horizontal tails simulates the potential elimination of a pair of primary pitching moment generating control effectors. The simulation is devised in such a manner that any combination of control effector failures can be easily implemented.

In addition, a direct comparison of the performance of each of the two control allocators in a reduced global position limits configuration is sought. In this investigation, the control effector position limits are redefined to be half of their original values. Due to the highly redundant control effector suite of the F-15 ACTIVE, the aircraft should still exhibit satisfactory performance in this configuration. It is desired to demonstrate the ability of MRA to utilize the full moment generating capabilities of a suite of controls. Similarly, it will be shown that the mixer currently on the F-15 ACTIVE does not take full advantage of the aircraft's control power, experiencing significant performance degradation in the reduced control deflection configuration. 


\subsection{Suggestions to the Reader}

This thesis covers a variety of research topics, some of which may be of greater interest to a given reader. For information regarding background on the F-15 ACTIVE control law as well as specifications for the aircraft the reader is referred to Chapter 2 as well as Appendix A. For general control allocation theory it is suggested that the reader begin with Chapter 3. The development of the new Bisecting, Edge-Searching Algorithm is confined to Chapter 4. Located towards the end of Chapter 4 are timing statistics associated with the Bisecting, Edge-Searching Algorithm. Background on the implementation of existing restoring methods as well as an introduction to variable step size minimum-sideforce restoring is presented in Chapter 5. For the reader who is primarily interested in the real-time piloted simulations and the control reconfiguration capabilities of MRA, results are given in Chapters 6 and 7 for each of these respective topics. Finally, a direct comparison between the capabilities of MRA and the original F-15 ACTIVE mixer with the aircraft in a reduced control deflection limits configuration is presented in Chapter 8. A summary of results and conclusions can be found in Chapter 9. 


\section{Chapter 2}

\section{F-15 ACTIVE}

\section{$2.1 \quad$ Introduction}

This chapter presents background information about the F-15 ACTIVE testbed aircraft that was utilized for this research. The objectives of the ACTIVE program are discussed as well as hardware modifications that have been made to the aircraft. In addition, a brief overview of the control law goals and format is undertaken, with an emphasis on how the control law is connected to the control allocator. The simulation code and environment is presented and the evaluation pilots involved with attaining the data for this research are introduced. Finally, the differences between simulation and actual flight are discussed.

\section{$2.2 \quad$ ACTIVE Objectives}

Thrust vectoring technology has been demonstrated successfully to provide maneuvering advantages in the slow, high angle-of-attack (AOA) flight regime. The primary objective of the F-15 ACTIVE test program was to expand the flight envelope in which useful thrust vectoring is available to enhance aircraft performance, maneuverability and controllability with production-representative nozzles. [6] In addition, ACTIVE seeks to investigate the utilization of non-traditional control effectors and the implementation of new concepts in control law/allocator design. The secondary objective of the ACTIVE program is the focus of the work contained herein. The implementation of the Bisecting, Edge-Searching Algo- 
rithm explores new possibilities in control allocation for tactical aircraft and is thus ideal for evaluation on the ACTIVE.

\subsection{Hardware}

The test aircraft is a modified pre-production F-15B on loan to NASA from the Air Force. This aircraft previously served as the testbed for the F-15 Short Takeoff and Landing / Maneuvering Technology Demonstrator (S/MTD) program. External modifications to the aircraft included the addition of two canards mounted on the upper inlet area forward of the wing. The canards themselves are modified F-18 stabilators. The aircraft is also equipped with two Pratt and Whitney F100-PW-229 turbofan engines modified to allow vectoring up to 20 degrees off of the normal engine thrust line in any direction. A quadruply-redundant, digital, fly-by-wire, flight control system is utilized in the aircraft. Figure 2.1 depicts the vital hardware components incorporated in the F-15 ACTIVE airframe. [6] Appendix A gives dimensions of the aircraft as well as a summary of the ACTIVE testbed experiments.

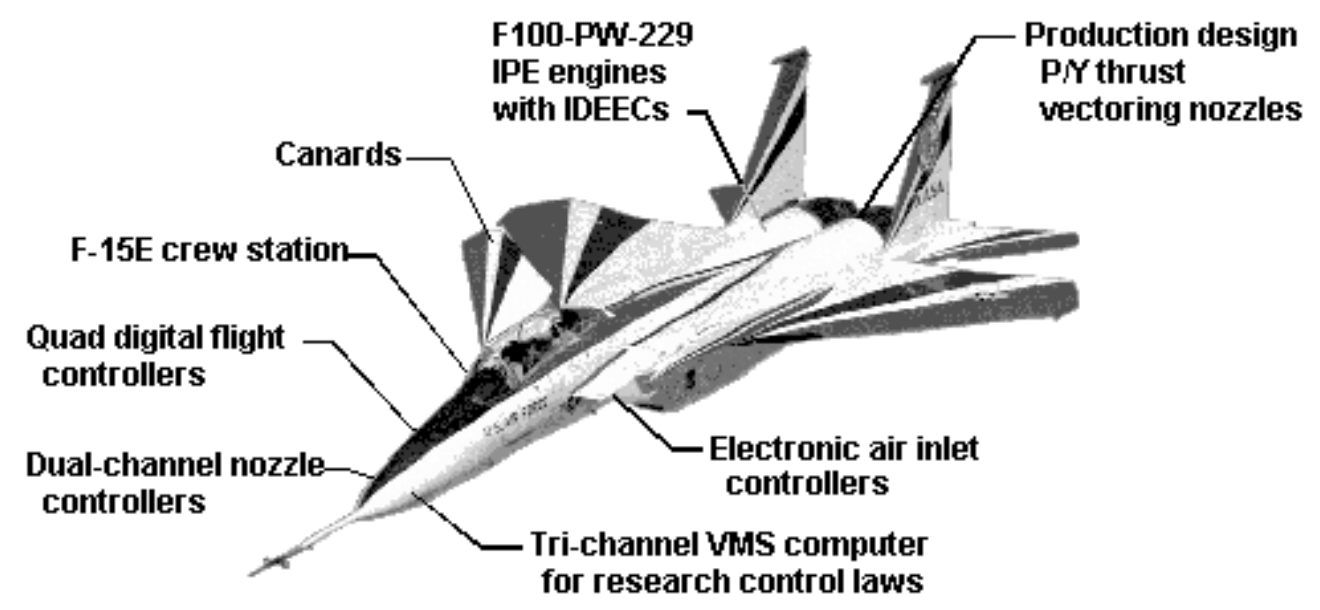

Figure 2.1: F-15 ACTIVE hardware components.

Table 2.1 lists the control effectors present on the F-15 ACTIVE and their associated position and rate limits. The surfaces listed are: DHT(L/R), left/right horizontal tails, 
DAIL(L/R), left/right ailerons, DCAN(L/R), left/right canards, DRUD(L/R), left/right rudders, $\mathrm{DTEF}(\mathrm{L} / \mathrm{R})$, left/right trailing edge flaps, DPV(L/R), left/right pitch thrust vectoring and DYV $(\mathrm{L} / \mathrm{R})$, left/right yaw thrust vectoring. All control deflections are defined positive trailing edge down. For yaw vectoring, jet plume left is defined as positive. The indicated control defection and rate limits were supplied by Boeing Phantom Works. The rate limits of the aerodynamic surfaces listed in Table 2.1 are without an externally applied aerodynamic loading.

Table 2.1: Control surface limits

$\begin{array}{lcc}\text { Control Effector } & \text { Deflection (deg) } & \text { Rate Limit }(\mathrm{deg} / \mathrm{s}) \\ \text { DHT(L/R) } & -29.0 / 15.0 & \pm 46 \\ \text { DAIL(L/R) } & -20.0 / 20.0 & \pm 100 \\ \text { DCAN(L/R) } & -35.0 / 15.0 & \pm 23 \\ \text { DRUD(L/R) } & -30.0 / 30.0 & \pm 105 \\ \text { DTEF(L/R) } & 0.0 / 35.0 & \pm 18 \\ \text { DPV(L/R) } & -20.0 / 20.0 & \pm 80 \\ \text { DYV(L/R) } & -20.0 / 20.0 & \pm 80\end{array}$

\subsection{Control Law}

\subsubsection{Objectives}

The basis for the F-15 ACTIVE control law is the set of aircraft equations of motion written in the stability axis system. Longitudinally, pitch stick commands angle-of-attack, $\alpha$, at low speeds and load factor, $n_{z}$, at high speeds. Logic is used near corner speed to blend angle-ofattack and $n_{z}$ command. The longitudinal control system utilizes both angle-of-attack and pitch rate feedbacks at low speeds. At higher speeds when load factor is commanded, $n_{z}$ is used as a feedback and the load factor error divided by the ratio $n_{z} / \alpha$ is used to provide an equivalent AOA error. Lateral stick commands a decoupled roll about the velocity vector. The yaw axis produces sideslip without roll; however, the rudder pedals are cross-fed to the lateral axis to allow the pilot to make minor bank angle corrections with rudder pedal inputs in the event that his hands are occupied with another task in the cockpit. [7] 
The control law design is based on the concept of equivalent systems. Flying qualities and equivalent systems research in the mid 1970's at McDonnell Douglas Aircraft (now Boeing) and elsewhere showed that augmented high order dynamics which did not closely approximate the responses of classical low order models generally received poor pilot ratings. Several control system approaches were developed in the 1980s to provide essentially decoupled, low order appearing responses. One such approach developed by McDonnell Douglas Aircraft (now Boeing), is based on a dynamic inversion format, and was applied in the mid 1990's to the F-15 ACTIVE aircraft. This "Versatile Control Augmentation System", described in reference 7 (McDonnell Douglas Corporation Proprietary) permits the direct specification of desired dynamics in terms of low order parameters such as frequencies, damping ratios and time constants. In common with other forms of dynamic inversion, this control law generates forward path commands in terms of desired angular accelerations $\left(\dot{P}_{c m d}, \dot{Q}_{c m d}\right.$ and $\left.\dot{R}_{c m d}\right)$, or desired Moments. This feature is particularly useful for control allocation research because most control allocation algorithms are based on determining the combination of control effector deflections that produce the desired set of commanded moments.

The control law is not presented at any level of high detail since it is proprietary to Boeing. In spite of not explaining the entire control law, it is the replacement of the control allocator with the Moment Rate Allocator that is the focus of this work, not the inner workings of the control law. Once an equivalent system model is defined, one can store the parameters associated with the desired aircraft performance in data tables as a function of flight condition. At each iteration in the control law, table look-ups can be used to determine the desired aircraft dynamics and insert them into the appropriate place in the control law algorithms. For instance, longitudinally, table look-ups are performed to find an equivalent short-period damping ratio and frequency that gives the desired aircraft response and meets the necessary level 1 handling qualities specifications found in section 3.2.2.1.2 of MIL-F-8785C. [8]

\subsubsection{Dynamic Inversion Format}

To ensure the dynamics of the Low Order Equivalent System, the control law designers have formatted the control law similar to a typical dynamic inversion control law. This type of control law is said to "subtract out the bad stuff" and "add in the good stuff". Dynamic inversion uses the control generated moments to cancel the unwanted dynamics of the aircraft and insert a desired response. [9] A linearization of the aircraft equations 
of motion is used to transform the nonlinear dynamics of the aircraft into a linear system in conjunction with feedback control. As stated in reference 10, "This approach enables direct incorporation of flying qualities objectives in the design process and can address the nonlinearities introduced by innovative control effectors and aircraft operation at extreme flight conditions." These dynamics are those that were defined by the equivalent systems concept. In dynamic inversion control laws, the aircraft equations of motion are considered to be of the form of equation 2.1

$$
\vec{f}(\dot{\vec{x}}, \vec{x}, \vec{u})=0
$$

where $\vec{x}$ are the states $\in \mathcal{R}^{n}$ and $\vec{u}$ are the control effectors $\in \mathcal{R}^{m}$. The control effectors are considered affine, meaning the control effectiveness slopes are assumed to be linear in nature but do not necessarily pass through the origin.

Figure 2.2 shows plots of the partial derivative of the rolling moment coefficient with respect to the left aileron deflection at 10,000 $\mathrm{ft}$ for different values of the Mach number. This figure is included to show that the control effectiveness data is, in general, linear in nature. Based on the assumption of the control effectors being affine, equation 2.1 can be rewritten as

$$
\vec{f}(\dot{\vec{x}}, \vec{x})+\vec{g}(\vec{x}) \vec{u}=0
$$

Linearize this equation with a first order Taylor series expansion about $\vec{x}_{r e f}$ and $\vec{u}_{r e f}$ such that $\dot{\vec{x}}_{r e f}=0$, (ie. steady flight), and rewrite to find

$$
\dot{\vec{x}}=\vec{f}(\vec{x})+\vec{g}(\vec{x}) \vec{u}
$$

The control $\vec{u}$ can be solved for by inverting $\mathrm{g}(\mathrm{x})$, assuming it is invertible, in equation 2.3 .

$$
\vec{u}=\vec{g}^{-1}(\vec{x})[\dot{\vec{x}}-\vec{f}(\vec{x})]
$$

If a set of desired dynamics $\dot{\vec{x}}_{d}$ is given, the control $\vec{u}^{\star}$ which produces these dynamics is found to be

$$
\vec{u}^{\star}=\vec{g}^{-1}(\vec{x})\left[\dot{\vec{x}}_{d}-\vec{f}(\vec{x})\right]
$$




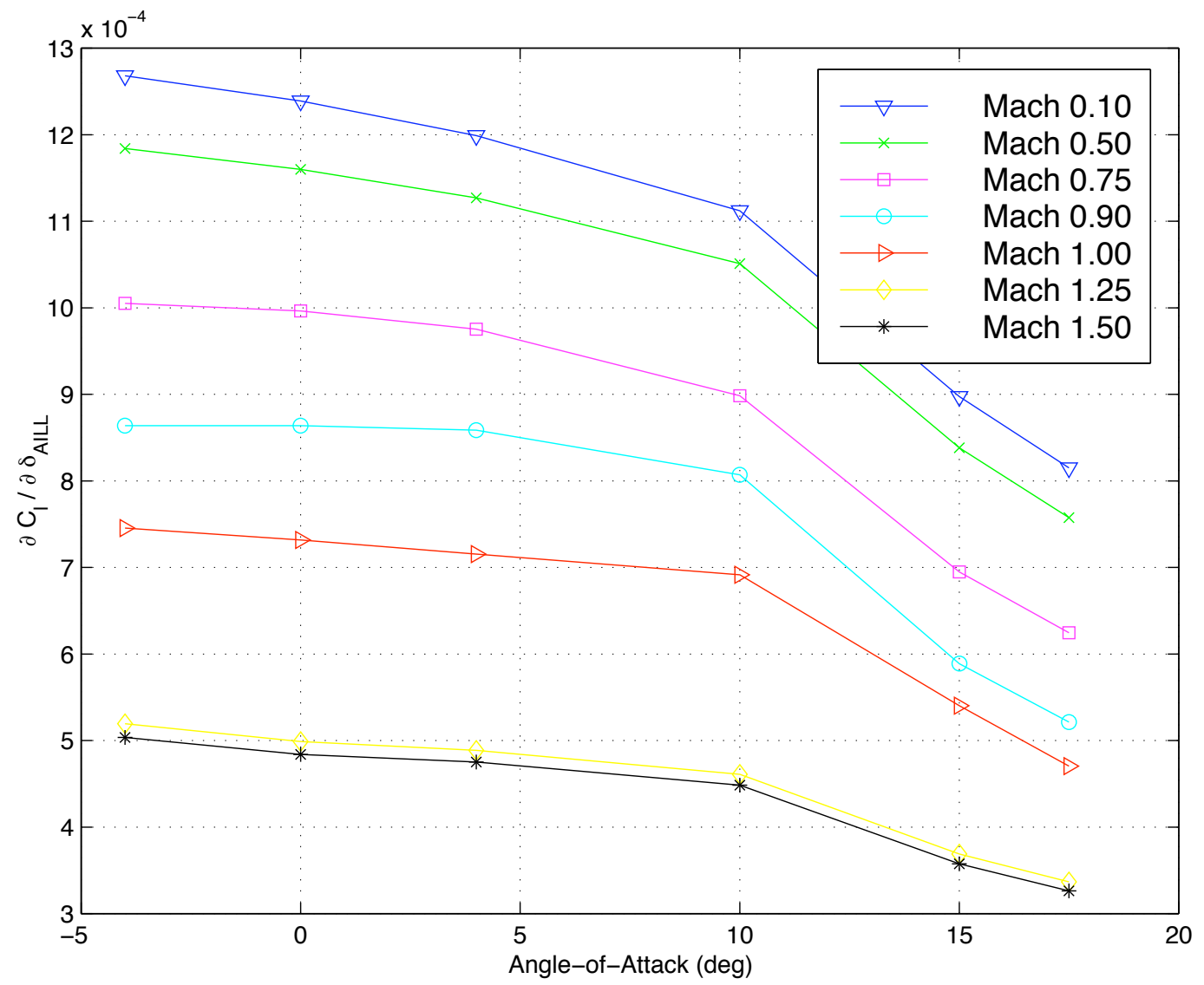

Figure 2.2: Affine control effectiveness data $\left(\partial C_{l} / \partial \delta_{A I L L}\right)$ as a function of Mach number for the F-15 ACTIVE at 10,000 $\mathrm{ft}$.

The calculation of $\vec{u}^{\star}$ from equation 2.5 assumes that the equations of motion are partitioned into three groups. The Kinematic equations of motion that are not associated with control effectors are defined as $\dot{\vec{x}}_{\text {Kinematic }}$. Related to the states directly influenced by the control effectors are $\dot{\vec{x}}_{\text {Controlled. Finally, the states that are indirectly influenced by the control }}$

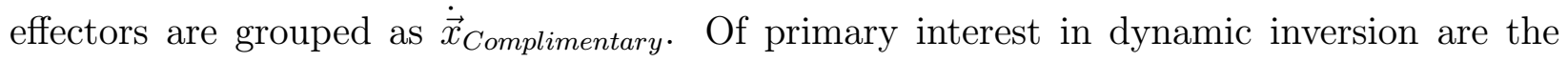
$\dot{\vec{x}}_{\text {Controlled }}$ states that relate directly to the aircraft pitching, rolling and yawing moments. If $\vec{u} \in \mathcal{R}^{m}$, and $m=3$, as in a classical aircraft, then $\vec{g}(\vec{x})$ is a square matrix that can be inverted. However, in modern tactical fighters, $m$ is approaching the order of 20 , and thus $\vec{g}(\vec{x}) \in \mathcal{R}^{3 \times 20}, \vec{g}(\vec{x})$ is no longer easily invertible. The control effectiveness matrix, $\vec{g}(\vec{x})$, often written as $\mathbf{B}$, is now a "wide" matrix with columns that are not necessarily linearly independent. Due to these properties of $\mathbf{B}$, a more sophisticated manner of determining the control solution $\vec{u}^{*}$ is needed. A control allocation method designed for multiply redundant 
control effectors, such as MRA, can be implemented in this situation to determine the set of control surface deflections $\vec{u}^{*}$ that produce the desired aircraft dynamics.

\subsubsection{Determing Desired Moments}

The outputs of the F-15 ACTIVE's control law, also referred to as the inputs to the control allocator, include commanded pitch, roll and yaw accelerations. These commanded angular accelerations can be converted to commanded moments. As a result, the F-15 ACTIVE's control law format is ideal for comparison of control allocation schemes in that the foundation of most control allocators lies in determining the feasible control combinations that will produce the set of desired aircraft moments. A schematic of the general format of the control law - control allocator framework is presented in Figure 2.3. In the figure, $\Omega$ represents the control inceptor space, $\delta_{S T I C K}$ indicates the actual control inceptor deflection, $\mathbf{m}_{\mathbf{d}}$ is the vector of desired aircraft moments, and $\Phi$ is the attainable moment subset (AMS). Although not the focus of this work, the interaction between the control law and control allocator is a topic of research which has recently developed a large amount of interest among the flight controls' community. As will be indicated below, the outputs of a given control law can be tailored to accommodate a particular form of a control allocator. In a sense, even though the control law and control allocator are depicted as separate blocks in Figure 2.3, there is coupling or overlap between the two blocks that can produce unexpected results if the control allocator is replaced with an allocator of a different format.

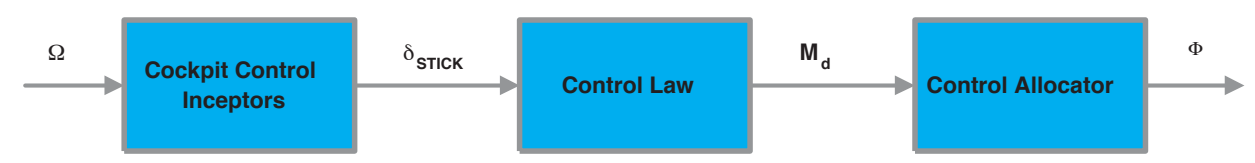

Figure 2.3: General format of control law - control allocator framework.

Study of the control law implementation revealed that the commanded accelerations were not calculated in the standard body-axis coordinate system in which $X_{B}$-axis points out the nose of the aircraft, $Y_{B^{-}}$axis out the right wing and the $Z_{B}$-axis completes the right-handed coordinate system. The commanded accelerations are given in what the control law designers define as the "stability primed" axis system. This use of a "different" coordinate system is a small example of the control law - control allocator interaction. This "stability primed" 
coordinate system is a special form of the standard body-axis system. Consider the aircraft in some reference flight condition, usually steady flight, so that the relative wind is seen from a constant direction by the aircraft. The $X_{S}$-axis is defined as the projection of the velocity vector of the aircraft relative to the air mass into the aircraft plane of symmetry. The $Y_{S}$-axis points out the right wing of the aircraft and the $Z_{S}$-axis completes the righthand rule. The primed portion of the "stability primed" description is believed to come from the assumption that the aircraft's cross-products of inertia are assumed to be zero in this system (ie. principal axes). Figure 2.4, taken from Dr. Wayne Durham's Aircraft Dynamics and Control class notes gives a graphical representation of the standard stability axis system. [11] The "stability primed" coordinate system is actually a combination of a body-carried and a body-fixed system. The standard stability-axis system is defined in a reference flight condition and from that point forth fixed to the body. The "stability primed" system is redefined at each iteration and is thus has characteristics of a body-carried system.

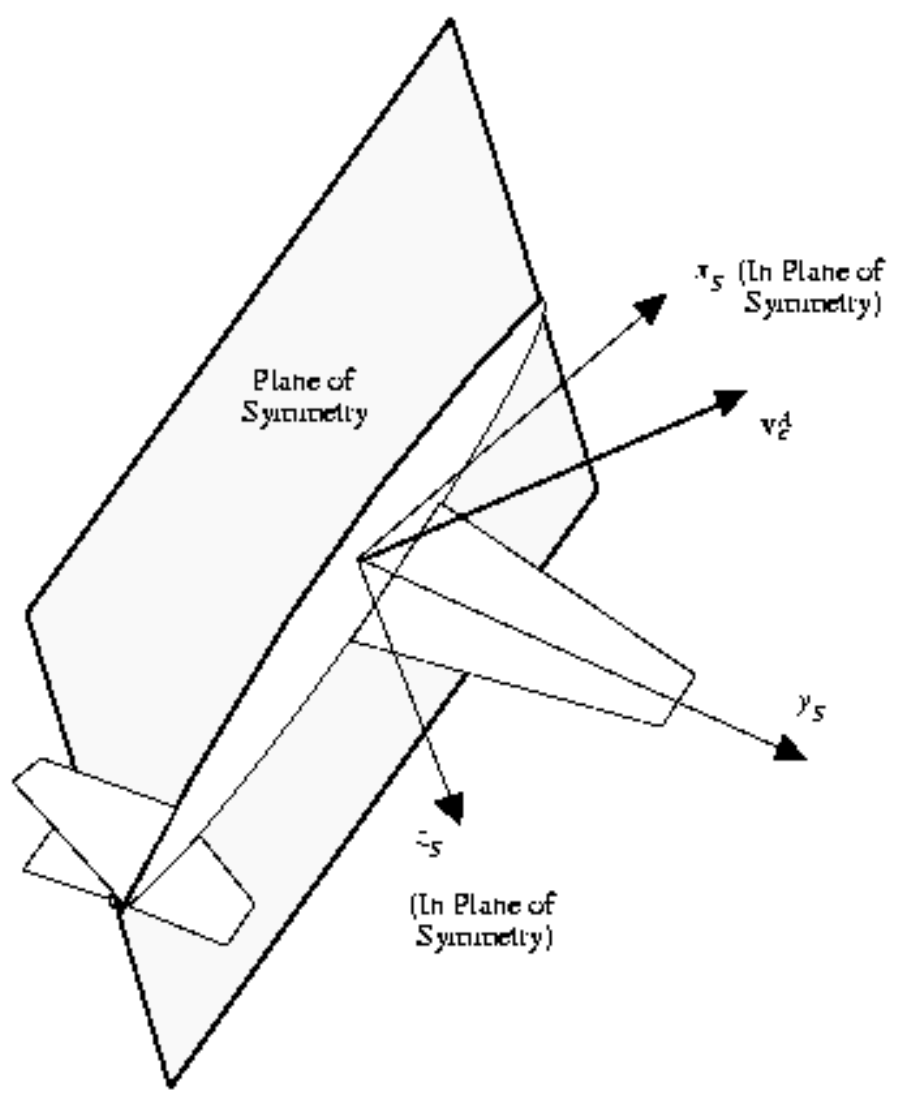

Figure 2.4: Standard stability-axis system. 
A simple stability-axis to body-axis coordinate transformation, as defined in equation 2.6, was used to translate the outputs of the control law into inputs for the control allocator. In this equation, the contribution of $\dot{\alpha}$ has been neglected.

$$
\left\{\begin{array}{l}
\dot{P}_{C M D} \\
\dot{Q}_{C M D} \\
\dot{R}_{C M D}
\end{array}\right\}_{B}=\left[\begin{array}{ccc}
\cos \alpha & 0 & -\sin \alpha \\
0 & 1 & 0 \\
\sin \alpha & 0 & \cos \alpha
\end{array}\right]\left\{\begin{array}{l}
\dot{P}_{C M D} \\
\dot{Q}_{C M D} \\
\dot{R}_{C M D}
\end{array}\right\}_{S}
$$

Once the accelerations were transformed, they were converted to moments through equation 2.7.

$$
\left\{\begin{array}{c}
C_{L_{\text {Desired }}} \\
C_{M_{\text {Desired }}} \\
C_{N_{\text {Desired }}}
\end{array}\right\}_{B}=\left\{\begin{array}{ccc}
\frac{I_{X X}}{\bar{q} S b} & 0 & 0 \\
0 & \frac{I_{Y Y}}{\bar{q} S \bar{c}} & 0 \\
0 & 0 & \frac{I_{Z Z}}{\bar{q} S b}
\end{array}\right\}\left\{\begin{array}{c}
\dot{P}_{C M D} \\
\dot{Q}_{C M D} \\
\dot{R}_{C M D}
\end{array}\right\}_{B}
$$

In equation 2.7, $C_{L_{\text {Desired }}}, C_{M_{\text {Desired }}}$ and $C_{N_{\text {Desired }}}$ are the desired non-dimensional rolling, pitching and yawing moment coefficients. The wing area is $\mathrm{S}$, the wing chord length is $\bar{c}$ and the dynamic pressure is $\bar{q}$. In addition, $I_{X X}, I_{Y Y}$ and $I_{Z Z}$ are the moments of inertia about the $X, Y$ and $Z$ axes, respectively. The remainder of the work contained herein is implemented using the stability-axis to body-axis transformation method.

\subsection{Simulation Code and Environment}

Using control system block diagrams and functional descriptions provided by Boeing Phantom Works, the F-15 ACTIVE control law and mixer were implemented in FORTRAN on a Silicon Graphics Origin 2000 ${ }^{T M}$ Deskside System. (180 MHz, 2 CPU, 256 MB, 4 GB disk) In addition, Boeing Phantom Works also provided aerodynamic and engine table look-up data for the simulation. The control law with mixer, aerodynamic and engine modules were formatted to be compatible with the CASTLE (Control Analysis and Simulation Test Loop Environment) architecture. CASTLE is a flexible, user-friendly 6 degree of freedom nonlinear aircraft simulation package that was originally developed by the Naval Air Warfare Center's Manned Flight Simulator Branch (MFS). [12] The flight simulation laboratory at Virginia Polytechnic Institute and State University currently utilizes CASTLE in its modified 2F122A 
motion based simulator. [13]

All simulations are run at a $100 \mathrm{~Hz}$ frame rate and data is recorded at $10 \mathrm{~Hz}$ unless otherwise stated. CASTLE can be run in both a batch and real-time mode. Canned inputs are inserted in the batch mode utilizing CASTLE's built in maneuver generator. The maneuver generator is capable of not only commanding standard doublet and step inputs, but also driving an input variable with a pre-determined time history of data. The real-time capabilities of the simulator are utilized with a pilot-in-the-loop performing a defined task. A calligraphic visual scene allows the pilot to fly the aircraft in a night/dusk environment. In addition, the inclusion of models for a naval air base, an aircraft carrier, and a formation or bogey aircraft produce an environment that promotes the evaluation of realistic flying tasks. The maneuver generator can also be used to drive the flight path of the bogey to produce realistic air combat maneuvering situations. The aircraft control stick feel and dynamics are produced utilizing an electronic control loader and can therefore be modified through software to be representative of the actual aircraft's stick feel. Since stick characteristics were not available for the F-15 ACTIVE, the characteristics of the F/A-18 E/F are utilized.

\subsubsection{Evaluation Pilots}

Two evaluation pilots were used to fly all data presented in this research. Several flights were flown by other pilots, but results from their flights were not included in the analysis. The first pilot will be referred to as subject pilot A from this point forth. Subject pilot A has a background in Navy fighters, carrier suitability and flight testing. He served as a test pilot instructor at the Navy Test Pilot School at Patuxent River Naval Air Base. In addition, subject pilot A is a member of the Society of Experimental Test Pilots. Subject pilot A is currently an Associate Professor in the Department of Aerospace and Ocean Engineering at Virginia Polytechnic Institute and State University and a senior member of the American Institute of Aeronautics and Astronautics.

The second pilot, subject pilot B, has no actual flight training time but has accumulated numerous hours of simulated flight time while working the Flight Simulation Laboratory at Virginia Tech. The majority of subject pilot B's simulated flight hours have been in the F-15 ACTIVE model that is the subject of this research. Subject pilot B has an undergraduate degree in Mechanical Engineering from the University of New Hampshire and is currently working on his Master of Science degree in Aerospace Engineering at Virginia Tech. He is 
a member of the American Society of Mechanical Engineers and the American Institute of Aeronautics and Astronautics.

\subsubsection{Handling Qualities Evaluation Using Real-Time Simulation}

Although real-time simulations offer a realistic flight environment, there are differences between actual flight and simulated flight that have a significant effect on handling qualities evaluation and task completion. These differences can enable a pilot with no actual flight time, subject pilot B, to consistently complete a maneuvering task in the simulator while a highly experienced and qualified test pilot, subject pilot A, has trouble with the task. On the other hand, if subject pilots A and B were to both be given a task in an actual aircraft, subject pilot A would surely outperform subject pilot B in almost every capacity. There is not a one-to-one correspondence between ground-based simulators and actual flight time.

The list of cues that are not available to a pilot in a ground-based simulation is extensive. In this research, no motion cues were used to offer the pilot a sensation of accelerations that he and the aircraft were experiencing. The calligraphic visual system utilized with its limited field of view forced the pilots to fly the tasks in a night/dusk environment that made aspect ratios, closure rate and ranges difficult to judge. In reference 14, Grantham compares ground-based simulations to in-flight simulations and actual flight time in terms of handling qualities. Grantham came to the following list of conclusions.

1. The effect of control sensitivity is more pronounced on a ground-based simulator than on an in-flight simulator.

2. In general, the pilot has a greater tendency to PIO an aircraft on a ground-based simulator than he does when flying an in-flight simulator.

3. Ground-based simulators are not adequate for assessing pilot/vehicle "performance" capabilities ... when the pilot has little depth/height perception from the outside scene presentation.

Grantham goes on to state that due to the differences between simulators and actual aircraft and the inability to account for all situations that can occur in the real world, the pilot cannot be exposed to "complete-tasks" operation of the aircraft in a simulator. [14]. The 
final ingredient that distinguishes actual flight time and simulated flight time is described by Grantham as the "pucker factor". This apprehension, emotional stress and responsibility that a pilot feels during actual flight time is not present during simulation. In fact, it is often the attitude of subject pilot B that "If I crash, a red light comes on, a bell rings, then I hit reset and try again." This type of attitude enables subject pilot $\mathrm{B}$ to be more aggressive than subject pilot A during tasks because subject pilot A had the "pucker factor" instilled in him over the course of his distinguished Navy flying career.

With the differences between simulation and actual flight time in mind, the reader is asked to consider that all the data contained in this research was attained through real-time piloted simulations. Furthermore, based on the backgrounds of subject pilots A and B, a distinction must be drawn between flights flown by each of these pilots. In general, data presented in this research from subject pilot B will demonstrate more aggressive maneuvering associated with the lack of ever having to worry about the "pucker factor". The inclusion of data from either pilot was based solely on the pilot's ability to complete the task, not the technique utilized during the task.

\subsection{Code Verification}

Before research could be performed on the F-15 ACTIVE, it was necessary to first verify the validity of the FORTRAN version of the control law and control mixer. Boeing Phantom Works provided a copy of their control law and mixer developed in Integrated Systems' MATRIX $_{X}$. Using the MATRIX $X_{X}$ AutoCoding capability, a version of the control law and mixer was produced in C. Input/Output wrappers were coded to interface the AutoCoded control law and mixer with the CASTLE architecture. The AutoCoded control law and mixer was thus the standard to which the FORTRAN version of the control law and mixer was compared. This comparison was performed by providing each control law and mixer with identical control inputs and monitoring the output of each model. To ensure validity of the entire control law and mixer, the comparison was performed at points throughout the flight envelope.

Several assumptions were made in the design of the input/output wrappers used for the $\mathrm{Au}$ toCoded implementation. These assumptions involved the interface of the variables already present in the CASTLE simulation software with the required inputs and outputs of the 
AutoCoded control law. Thus, it is possible that the AutoCoded version was not completely representative of the actual aircraft control system. Nevertheless, both of the versions of the control system produced aircraft dynamics that were representative of an actual aircraft. This level of verification is sufficient for the type of research being performed in this paper. Although it seems counter-intuitive to take the time to code the control law and mixer in FORTRAN and then verify it, it was decided that coding it by hand would produce far more efficient code. Efficiency of the code is a key factor in any timing analysis to be performed on the mixers. Hand coding simplifies insertion of a new control allocator by coding the control law and control mixer in separate modules. The independent control law - control allocator block diagram concept, described in Figure 2.3, was used as a foundation for the coding structure. 


\section{Chapter 3}

\section{Control Allocation Theory}

\subsection{Allocation Methods}

The control allocation method implemented in this research represents the current state of the work originally performed by Bordignon and Durham [15-18] and later refined by Bolling [2] and then Leedy [1]. In this method, consider $m$ control effectors $\mathbf{u}$ that produce $n$ objectives $\mathbf{y}, m>n$, through the linear mapping $\mathbf{f}: \mathcal{R}^{n} \rightarrow \mathcal{R}^{m}, \mathbf{y}=\mathbf{f}(\mathbf{u})$. Each of the control effectors has global minimum and maximum position limits, $u_{i_{\min }}$ and $u_{i_{\max }}$. The minimum and maximum values of each control effector define the admissible control subset,

$$
\Omega=\left\{\mathbf{u} \in \mathcal{R}^{m} \mid u_{i_{\min }} \leq u_{i} \leq u_{i_{\max }}\right\} \subset \mathcal{R}^{m}
$$

If the objective, $\mathbf{y}$, is specified, control allocation is the determination of an inverse mapping $\mathbf{g}: \mathcal{R}^{m} \rightarrow \mathcal{R}^{n}$, such that $\mathbf{u}=\mathbf{g}(\mathbf{y})$. In aircraft control law design, the objective of primary interest is the vector of desired control-generated moments, $\mathbf{m}_{d}$. These desired moments are related to the allocated control effectors through the $3 \times m$ control effectiveness matrix. The control effectiveness matrix is usually represented by the symbol, $\mathbf{B}$. The components of this matrix are defined by equation 3.2 ,

$$
\begin{aligned}
\mathbf{B}_{i, j} & =\frac{\partial C_{m_{i}}}{\partial u_{j}} \\
\mathbf{m} & =\mathbf{B u}
\end{aligned}
$$


where $C_{m_{i}}$ represents the aircraft non-dimensional moment coefficients. The subset of attainable moments $\Phi$ is the image of $\Omega$, and for all $\mathbf{u}$ that lie within $\Omega, \mathbf{m}=\mathbf{B u}$ is a subset of $\Phi$. Thus the three-moment optimal control allocation problem can be stated as, given the control effectiveness $\mathbf{B}$, the subset of admissible controls, $\Omega$, and a half-line $\ell$ in the direction of a desired moment $\mathbf{m}_{\mathbf{d}}$, find the intersection of $\ell$ with $\partial(\Phi)$, the convex hull of $\Phi$. The algorithms used to determine this intersection are outlined in chapter 4.

\subsection{Frame-wise Control Allocation}

In this research, control allocation has been performed on a frame-wise basis in order to take into account the rate limits on the control surfaces. The control effectors also have limits on rates of travel, $\dot{\mathbf{u}}_{\min }$ and $\dot{\mathbf{u}}_{\max }$. Assuming these rate limits hold constant over a specified time period, $\Delta t$, limitations are placed on the travel distance of the controls, $\Delta \mathbf{u}_{\min }=\dot{\mathbf{u}}_{\min } \Delta t$ and $\Delta \mathbf{u}_{\max }=\dot{\mathbf{u}}_{\max } \Delta t$. Thus, the subset of admissible controls over a given time period $\Delta t$ is the more restrictive of the global position and rate limits. Furthermore, it is assumed that the mapping $\mathbf{f}$ holds for a change in control position over the time period $\Delta t$, ie. $\mathbf{y}=\mathbf{f}(\mathbf{u})$ and $\Delta \mathbf{y}=\mathbf{f}(\Delta \mathbf{u})$. The change in controls required, $\Delta \mathbf{u}$, to obtain the desired change in moment, $\Delta \mathbf{m}_{\mathbf{d}}$, is calculated through the Moment Rate Allocation algorithms discussed in chapter 4 .

In the work previously performed by Leedy and Durham [1], control effectiveness was evaluated on a frame-wise basis and assumed to be linear in that local region. Thus, on a frame-wise basis, equations 3.2 and 3.3 become,

$$
\begin{aligned}
\mathbf{B}_{k} & =\left[\frac{\partial m}{\partial u}\right]_{k} \\
\Delta \mathbf{m}_{d_{k}} & =\mathbf{B}_{k} \Delta \mathbf{u}_{k} \\
\Delta \mathbf{m}_{d_{k}} & =\mathbf{m}_{d_{k+1}}-\mathbf{m}_{k}, \\
\mathbf{u}_{k} & =\mathbf{u}_{k-1}+\Delta \mathbf{u}_{k}
\end{aligned}
$$

Based on the above equations, the control allocator first calculates $\Delta \mathbf{m}_{d_{k}}$ and then evaluates the local Jacobian matrix $\mathbf{B}_{k}$. The change in control surface position for that time step, $\Delta \mathbf{u}_{k}$, is the output of the control allocator. $\Delta \mathbf{u}_{k}$ is determined so that it will not violate either the position or rate constraints enforced on the control surface. 
In this work, it was determined that, for computational efficiency, control effectiveness would be stored in data tables and looked-up for the current flight condition. The assumption was made that at a particular flight condition, the control effectiveness was linear throughout the range of motion of each control. Linearization was thus performed about the origin of the control deflection, $u=0$. This assumption means that the $\mathbf{B}_{k}$ in equation 3.4 is the global control effectiveness matrix for the given flight condition. Determination of the global control effectiveness matrix involved a sweep through a set of pre-determined breakpoints for angle-of-attack, altitude, Mach number and power lever angle. Power lever angle sweeps were only performed on the thrust vectoring controls. In addition, data was collected for both a clean and a powered approach configuration. The breakpoints used in the simulation code are given in Table 3.1. The power lever angles chosen for breakpoints, 104.0, 118.0 and 127.0 degrees correspond to full military power, half afterburner and full afterburner, respectively. These breakpoint values are the same as those utilized in the engine table look-ups.

A sample plot of the rolling moment due to the left horizontal tail at an altitude 10,000 ft for different values of Mach and angle-of-attack was shown in Figure 2.2. From this figure, note that the effectiveness data is essentially linear in nature. The effectiveness data for the different moment and control effector combinations in general followed the same linear trend.

Table 3.1: Control effectiveness breakpoints.

\begin{tabular}{|l|c|c|c|}
\hline Configuration & Variable & Units & Breakpoints \\
\hline \hline Clean & Alpha & $d e g$ & $-4.0,0.0,4.0,10.0,15.0,17.5$ \\
\hline PA & Alpha & $d e g$ & $-4.0,0.0,4.0,10.0,15.0,17.5$ \\
\hline Clean & Mach & $n d$ & $0.1,0.5,0.75,0.90,1.0,1.25,1.5$ \\
\hline PA & Mach & $n d$ & $0.1,0.15,0.2,0.4,0.6,0.8$ \\
\hline Clean & Altitude & $f t$ & $100.0,10000.0,20000.0,30000.0$ \\
\hline PA & Altitude & $f t$ & $100.0,500.0,1000.0,1500.0,2000.0,3000.0,5000.0$ \\
\hline Clean & PLA & $d e g$ & $20.0,50.0,83.0,94.0,104.0,118.0,127.0$ \\
\hline PA & PLA & $d e g$ & $20.0,50.0,83.0,94.0,104.0,118.0,127.0$ \\
\hline
\end{tabular}

The linearization code used to extract the control effectiveness data and a description of how the code works is given in Appendix B. 


\subsection{Direct Allocation Solution}

The solution to the 3-moment optimal control problem involves the determination of the intersection of the half-line, $\ell$ with $\partial(\Phi)$, the convex hull of $\Phi$. However, the boundary of $\Phi$ is not easily found. The geometry of the attainable moment subset for the 3-dimensional problem is in general the projection of an $m$-dimensional rectangular box (where $m$ is the number of control effectors) into three dimensions. Unfortunately, the boundary, $\partial(\Phi)$, is not simply the image of $\partial(\Omega)$. This inconsistency between $\partial(\Phi)$ and $\partial(\Omega)$ results because some points of $\partial(\Omega)$ map to the interior of $\Phi$. Thus one must determine which points on $\partial(\Omega)$ map to $\partial(\Phi)$. The determination of the boundary of $\Phi$ is what is commonly known as the convex hull problem. However, the determination of $\partial(\Phi)$ is not the focus of this work. For more information on the geometric techniques used to find $\partial(\Phi)$, the reader is referred to Durham. [4]

Once $\partial(\Phi)$ is found, one must determine the edge or facet to which $\mathbf{m}_{\mathbf{d}}$ points. It is on this edge or facet that one can find the maximum attainable moment in the direction of $\mathbf{m}_{\mathbf{d}}$. After finding the correct facet, the intersection of $a \hat{\mathbf{m}}_{\mathbf{d}}, a>0, \hat{\mathbf{m}}_{\mathbf{d}}$ a unit vector in the direction of $\mathbf{m}_{\mathbf{d}}$, with the facet or edge is calculated. If $a \geq\left|\hat{\mathbf{m}}_{\mathbf{d}}\right|$, (ie. $a \geq 1$ ) the desired moment is attainable, otherwise the control system is commanding a moment that the aircraft is not physically capable of achieving. Once linear algebra techniques have been used to find the intersection point, the combination of controls that generate that point must be found. The intersection is the vector sum of one of the vertices that defines the facet and some positive fractional part of two of the vectors that comprise the facet. The controls that generate that point in $\Phi$ can be determined by adding the corresponding portions of the control vectors from $\Omega$. If $a=\left|\hat{\mathbf{m}}_{\mathbf{d}}\right|$, the solution vector has been found in the previous step.(ie. the solution is on the boundary of $\partial(\Phi))$ However, if $a>\left|\hat{\mathbf{m}}_{\mathbf{d}}\right|$, the controls obtained from the previous step must be scaled down by a factor of $K=\left|\hat{\mathbf{m}}_{\mathbf{d}}\right| / a$ to obtain a solution. In the case that $a<\left|\hat{\mathbf{m}}_{\mathbf{d}}\right|$, the moment is not attainable. For this situation, the solution of the boundary of $\Phi$ is used to preserve the direction of the desired moment.

Direct allocation implemented on a frame-wise basis by definition guarantees that the entire AMS and the full $\triangle \mathrm{AMS}$, the portion of the AMS attainable at each iteration due to rate limits on control surfaces, will be used in the determination of the control effector solution. In reference 5, Durham stated, "Direct allocation is guaranteed to fully exploit the moment generating capabilities of a given control configuration". A new version of the Cascaded 
Generalized Inverse, CGI, is currently under development that is projected to guarantee the use of the entire AMS. However, this CGI has a computational complexity that increases quadratically with the number of control effectors. In addition, Moment Rate Allocation was the only control allocation method found in the literature that explicitly accounted for rate as well as position limits on the control effectors. It is primarily for these reasons that further investigation into the Moment Rate Allocation has been pursued in this research. Other allocation methods including Cascaded Generalized Inverses [19] and Daisy-Chaining [15] exist in the literature; however, references $3,5,15$ and 19, to name a few, have repeatedly demonstrated advantages of using the Direct Allocation on a frame-wise basis over other existing methods. The one caveat has always been the fact that the Direct Allocation algorithms were computationally intensive. The new edge-searching algorithm presented in the following chapter greatly reduces the complexity of the searching algorithm and ultimately eliminates the final hurdle on the track to implementation of MRA on an actual aircraft. 


\section{Chapter 4}

\section{Bisecting, Edge-Searching Algorithm}

\subsection{Background}

The goal of the control allocation algorithms is to determine the facet or edge of the attainable moment subset that the half-line $\ell$ in the direction of the desired moment intersects. Once the proper facet has successfully been located, one can then determine the combination of control effectors that define this facet and allocate them to generate the desired moment. All work done by Durham, Bordignon, Bolling and Leedy in direct allocation up to this point utilized a brute-force facet-searching method to determine this intersection. The algorithm involved looking at facets defined by a pair of controls and tested to see if $\ell$ intersected the facet. Despite efforts to find a way to ensure that the solution be found quickly, there was no guarantee that the intersection would not be found in the last pair of facets evaluated. This potential for having to search the entire AMS for the solution meant that, in general, searching $m$ controls two at a time the required number of floating point operations varied as $m(m-1)$, and could be very high. For implementation of this method, the reader is referred to 2,16 and 19 .

As the number of controls utilized on a modern tactical aircraft nears 20 and promises to continue increasing, one begins to become concerned about brute-force facet-searching floating point operations on the order of $\mathrm{m}^{2}$. Thus, a more computationally efficient method of determining the correct facet would be beneficial to the minimization of floating point operations required in the implementation of the direct allocation method. The following sections give a description of one such method that was developed for and implemented in 
the real-time simulations performed in this paper. The foundation for the method presented herein is found in reference 3. It will be shown through Matlab simulation of arbitrary control effectiveness matrices and random desired objectives that the computational complexity of this algorithm increases linearly with $m$. A linear relationship between the number of control effectors and the associated number of floating point operations, rather than the original quadratic relationship, is a significant savings as $m$ grows large.

\subsection{Two-Dimensional Problem}

For the purpose of understanding the concepts involved with a particular method, it is often easier to begin a problem description in lower dimensions and then work up to the more complicated higher dimensions. With this idea in mind, the solution to a two-moment problem can offer a great deal of insight into the three-moment problem. As a forewarning, note that the terms moment and objective will be used interchangeably in the description that follows. The use of the term moments is specific to the problem of aircraft control allocation while the term objective is indicative that the theory behind the Bisecting, EdgeSearching Algorithm can be applied to non-aircraft specific problems.

The geometry of the two-dimensional problem is $\mathbf{m}_{\mathbf{d}} \in \mathcal{R}^{2}$. Figure 4.1 shows the attainable pitching and yawing moments for the F-15 ACTIVE with twelve controls at a flight condition of 400 knots and 10,000 ft. The $C_{m}$ and $C_{n}$ axis, corresponding to the non-dimensional pitching and yawing moment coefficients, are replaced in the figure by the $x$ and $y$ axis, respectively.

In this two-dimensional problem, the control effectiveness matrix $\mathbf{B}$ is comprised of only two rows. Therefore, $\mathbf{B} \in \mathcal{R}^{2 \times m}$, and can be written as two row vectors,

$$
\mathbf{B}=\left[\begin{array}{l}
\mathbf{r}_{1} \\
\mathbf{r}_{2}
\end{array}\right]
$$

The first step in the solution is based on the observation that one can find the four vertices of $\Phi$ with the minimum and maximum $x$-components and the minimum and maximum $y$ components. The determination of these vertices is done by examining the signs of the entries of $\mathbf{r}_{1}$ and $\mathbf{r}_{\mathbf{2}}$. The $x$-component for any $\mathbf{u}$ is given by $\mathbf{r}_{\mathbf{1}} \mathbf{u}$, the dot product of the first row of $\mathbf{B}$ and the vector of control positions, $\mathbf{u}$. If the maximum value is sought for a given row, one 


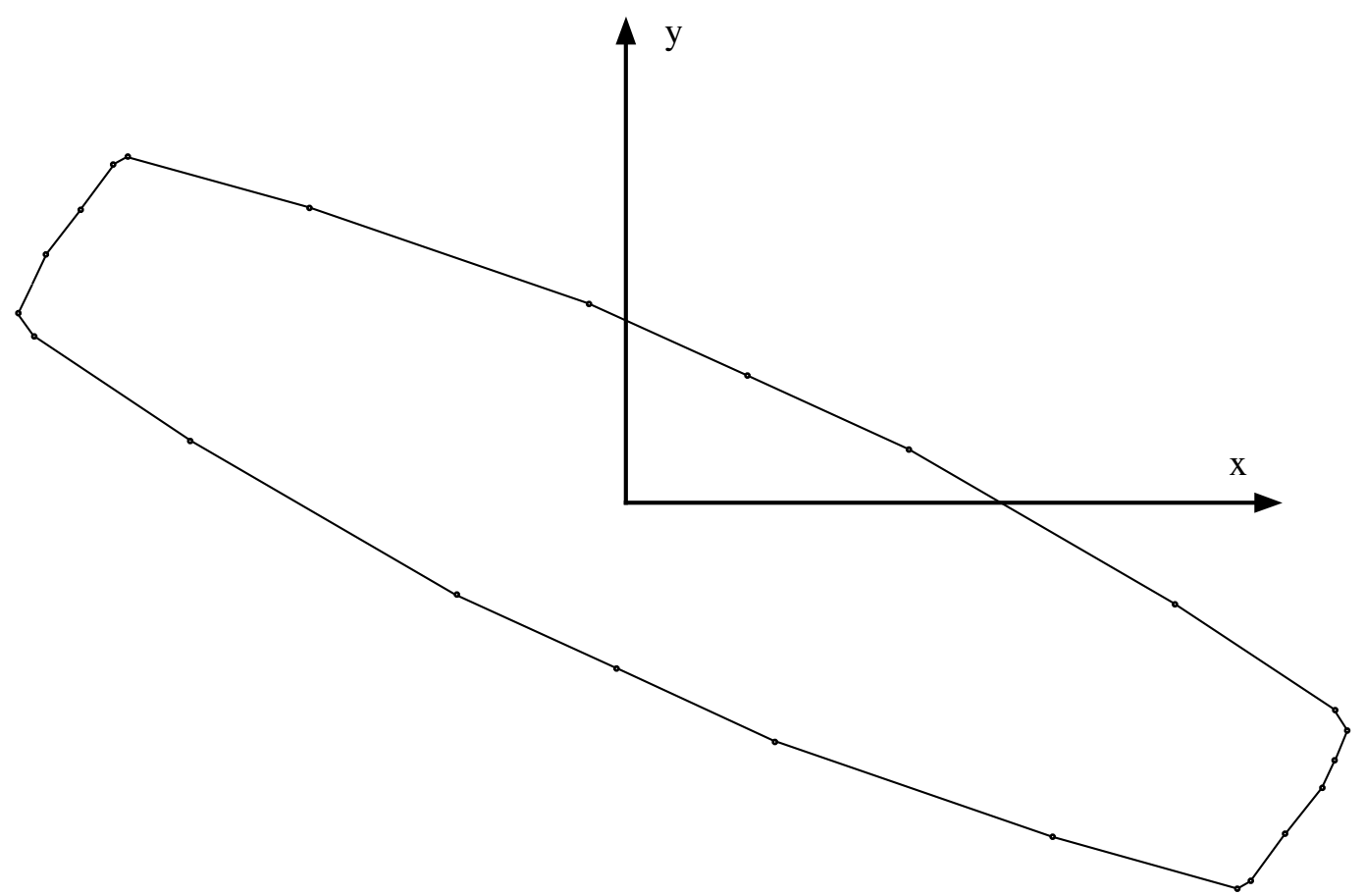

Figure 4.1: Two-dimensional AMS for the F-15 ACTIVE. Small dots represent the vertices of the AMS. $x$ and $y$ axes correspond to non-dimensional pitching and yawing moment coefficients, respectively.

places the individual controls at their positive stops if the row entry has a positive sign and at their minimum stops if the sign of the row entry is negative. The opposite is true if the minimum is desired. In this work, the researcher is primarily concerned with the maximum $x$-component. For $\mathbf{r}_{1}$, the vector of controls that produces the maximum $x$-component can be denoted by $\mathbf{u}_{x, \text { Max }}$. This vertex corresponding to this combination of controls is indicated by a large red dot in Figure 4.2 for the attainable moments from Figure 4.1. The coordinates of the vertex with the maximum $x$-component are therefore given by $\mathbf{y}_{x, \operatorname{Max}}=\mathbf{B} \mathbf{u}_{x, \operatorname{Max}}$. In the aircraft specific problem $\mathbf{y}_{x, \text { Max }}=\mathbf{m}_{\mathbf{d} x, \text { Max }}=\mathbf{B u}_{x, \text { Max }}$. The other three previously mentioned vertices can be found accordingly if needed. From this point on, to ease the confusion of notational differences, only non-aircraft specific notation will be considered and the desired objective/moment will be referred to as $\mathbf{y}_{\mathbf{d}}$. However, all figures used in the explanation of the method are representative of an actual aircraft problem.

As expected, knowledge of vertices does not offer much insight into the the geometry of the problem. Nevertheless, the connection of two vertices to form an edge is useful. This concept will be repeatedly used in the remainder of this description. The next step in the solution 


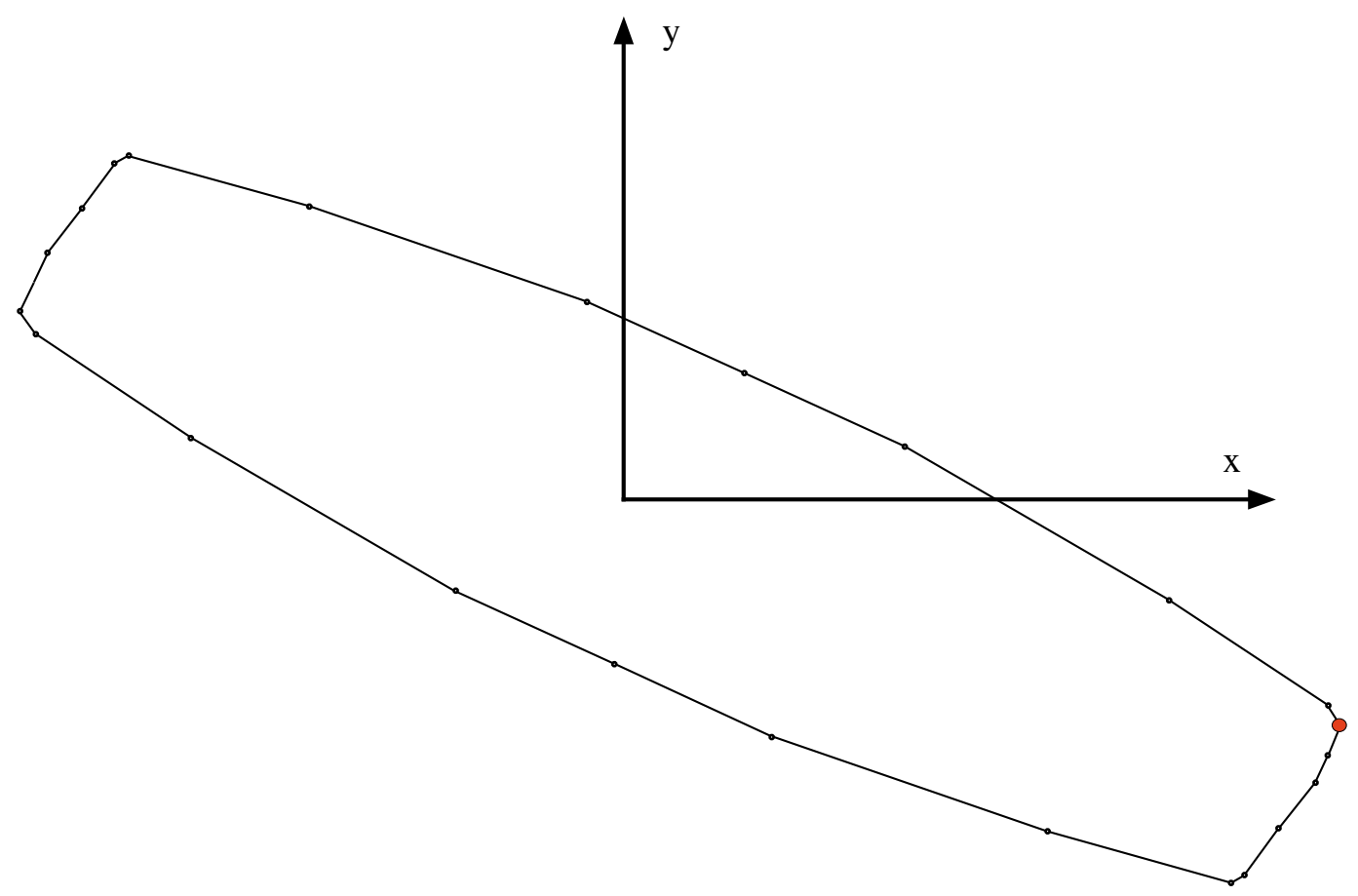

Figure 4.2: Identification of $\mathbf{y}_{x, M a x}$.

to the two-dimensional problem is to rotate the $x-y$ axes through an angle $\phi$. After locating $\mathbf{u}_{x, \text { Max }}$, the resulting control effectiveness matrix is given by equation 4.2 ,

$$
\mathbf{B}^{\prime}=\left[\begin{array}{l}
\mathbf{r}_{1}^{\prime} \\
\mathbf{r}_{2}^{\prime}
\end{array}\right]=T \mathbf{B}
$$

where the rotation matrix $\mathrm{T}$ is an orthonormal matrix given by equation 4.3.

$$
T=\left[\begin{array}{cc}
\cos \phi & \sin \phi \\
-\sin \phi & \cos \phi
\end{array}\right]
$$

As the axis rotate, the signs of the entries in $\mathbf{r}_{\mathbf{1}}^{\prime}$ will change, identifying new controls $\mathbf{u}_{x, \text { Max }}^{\prime}$ and corresponding coordinates for $\mathbf{y}_{x, \text { Max }}^{\prime}$ as functions of the rotation angle $\phi$. These new controls and associated coordinates will be referred to as $\mathbf{u}_{x, \text { Max }}^{\prime}(\phi)$ and $\mathbf{y}_{x, \text { Max }}^{\prime}(\phi)$, respectively. The angle $\phi_{1}$ at which the first such sign change occurs identifies the controls $\mathbf{u}_{x, \text { Max }}^{\prime}\left(\phi_{1}\right)$ and the vertex $\mathbf{y}_{x, \text { Max }}^{\prime}\left(\phi_{1}\right)$ that is connected to the original $\mathbf{y}_{x, \text { Max }}^{\prime}$ by an edge. [3] Figure 4.3 shows the identification of the first edge. In Figure 4.3, the original $\mathbf{y}_{x, M a x}^{\prime}$ is indicated by a large red dot and the vertex that forms the first edge is shown as a large black dot. If one 
were to continue to increase $\phi$ around a full $360^{\circ}$ sweep, the entire geometry of $\partial(\Phi)$ could be found.

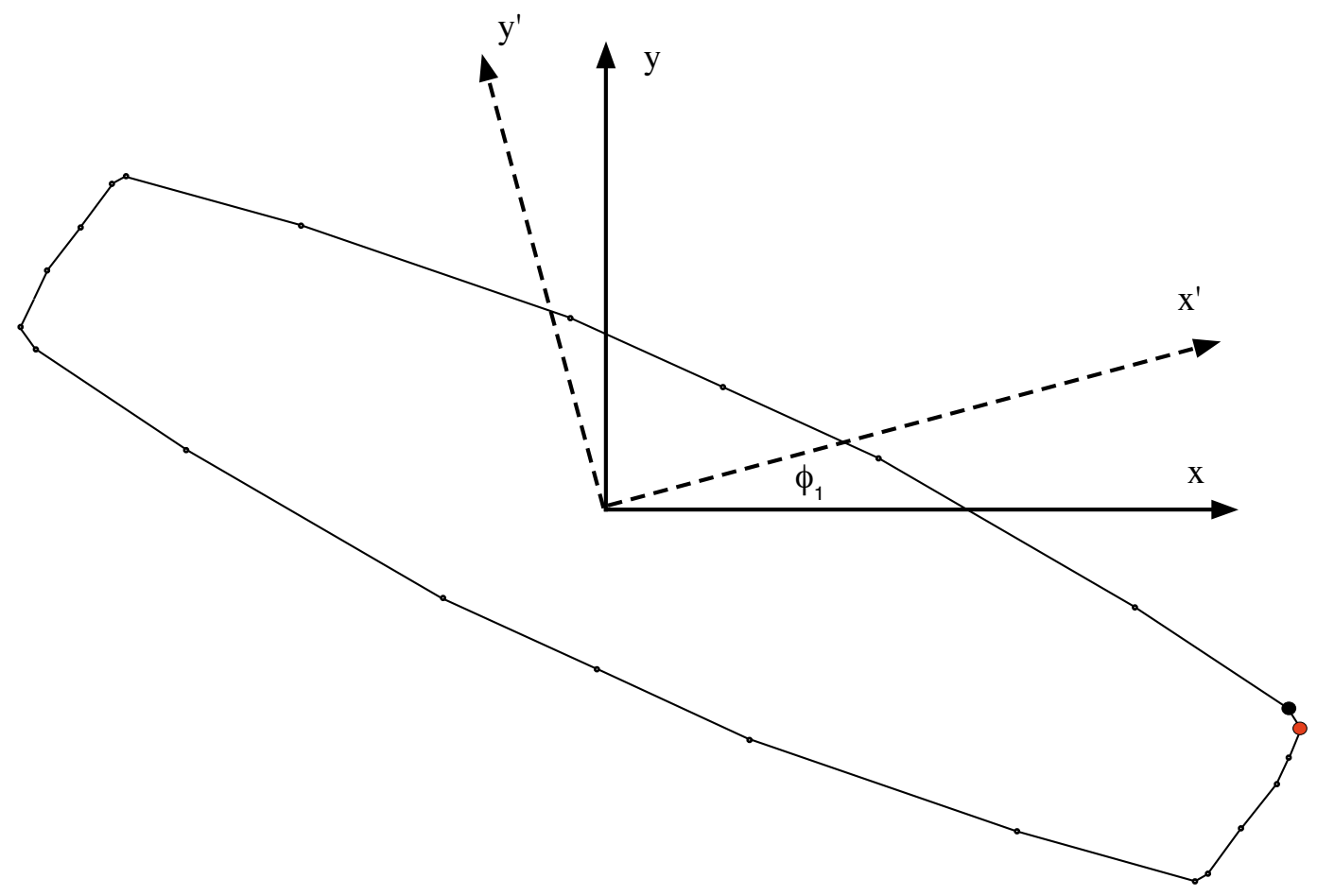

Figure 4.3: Rotation by $\phi$ to determine $\mathbf{y}_{x, \operatorname{Max}}^{\prime}$.

However, varying $\phi$ and observing when signs of entries of $\mathbf{r}_{1}^{\prime}$ change could prove to be a tedious bookkeeping problem. Instead, if one analytically determines the angles at which the entries of $\mathbf{r}_{\mathbf{1}}^{\prime}$ go to zero, from this point the sign of that particular entry will change if the angle $\phi$ is slightly increased or decreased. Furthermore, one can state that a zero in the $i^{\text {th }}$ entry of $\mathbf{r}_{\mathbf{1}}^{\prime}$ indicates that $x^{\prime}$ is perpendicular to the edge in $\Phi$ that corresponds to a mapping of the edge in $\Omega$ defined by the $i^{t h}$ control. This edge is defined to be on the boundary of $\Phi$. Simply stated, a zero in the $i^{\text {th }}$ entry of $\mathbf{r}_{\mathbf{1}}^{\prime}$ is directly related to the definition of the $i^{\text {th }}$ control in $\Omega$.

Define the angle at which the $j^{\text {th }}$ entry of $\mathbf{r}_{1}^{\prime}, r_{1, j}^{\prime}$, goes to zero, as $\phi_{j}$. Transformed by the matrix $\mathrm{T}$ through an angle $\phi_{j}, r_{1, j}^{\prime}$ is given by,

$$
r_{1, j}^{\prime}=r_{1, j} \cos \phi_{j}+r_{2, j} \sin \phi_{j}=0
$$


Solving for $\phi_{j}$, one finds

$$
\phi_{j}= \pm \arctan 2\left(-r_{1, j} / r_{2, j}\right)
$$

A list of $2 m$ angles that define the vertices of $\Phi$ can be calculated by finding $\phi_{j}, j=1 \ldots m$. Since these angles are generated by cycling through the list of controls, these vertices will be in order of control number. In order to utilize this list to determine the edges that define $\partial(\Phi)$, the list must be sorted by angle from least to greatest in the range $0 \leq \phi_{j} \leq 2 \pi$. The sorted indices indicate the controls associated with each edge proceeding counter-clockwise from the original vertex, $\mathbf{y}_{x, \operatorname{Max}}$. This list constitutes sufficient information to reconstruct the complete geometry of $\Phi$. [3]

However, in order to reduce the computational complexity of the direct allocation solution, it is imperative that one is not required to generate the entire AMS geometry. With this in mind, if the problem can somehow be restated or transformed in such a manner that only a small portion of the geometry is necessary to determine the solution edge, the problem is greatly simplified. Consider a transformation that causes the desired moment/objective to lie along the $x$-axis, that is the half-line $\ell$ is coincident with the positive $x$-axis. A simple transformation matrix similar to that found in equation 4.3 can be found that produces this alignment. A representation of the rotated AMS is given in Figure 4.4.

Once rotated, the optimal control allocation problem can be solved by simply determing which edge crosses the $x$-axis. This edge is characterized by a change in sign of the $y$ component as one proceeds from one vertex to the next. In order to move in the proper direction, if the $y$-component of the original $\mathbf{y}_{x, \text { Max }}$ is negative, one moves in an counterclockwise direction, calculating the sign of the $y$-component of each vertex in the sorted list until the sign changes. At this point, one has determined the two vertices that define the solution edge. Similarly, if the $y$-component of the starting vertex is positive, one proceeds with the same methodology, but in a clock-wise direction. The resulting edge for the problem defined in Figure 4.1 is highlighted in red in Figure 4.4. The two-dimensional problem is now solved.

To summarize, the two-dimensional optimal allocation problem can thus be solved by stepping through the following list:

1. Orient the AMS such that the half-line $\ell$ is coincident with the positive $x$-axis. 


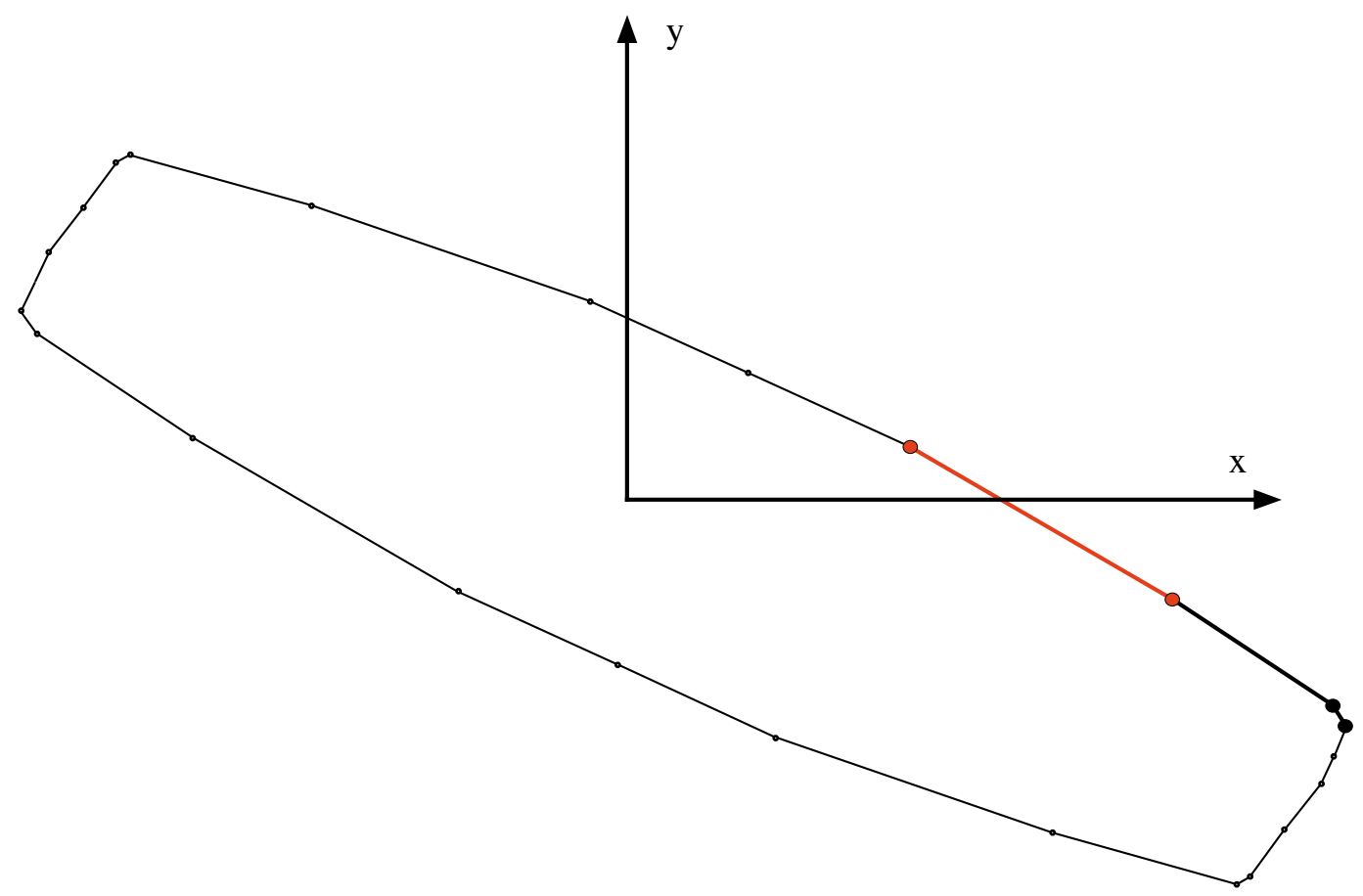

Figure 4.4: Rotated AMS with $\mathbf{m}_{\mathbf{d}}$ aligned with $x$-axis. Solution edge determined by finding a change in sign of the $y$-component of two consecutive vertices.

2. Determine the vertex that corresponds to the maximum $x$-component of the objective, $\mathbf{y}_{x, \operatorname{Max}}=\mathbf{m}_{\mathbf{d} x, \operatorname{Max}}=\mathbf{B} \mathbf{u}_{x, \operatorname{Max}}$.

3. Determine the angles, $\phi_{j}, j=1 \ldots m$, that correspond to each of the control effectors.

4. Sort the control effectors by the angles $\phi_{j}$ from the least to the greatest

5. Determine the sign of the $y$-component of the starting vertex, $\mathbf{y}_{x, M a x}$. If the sign from step 5 is positive, move in a clockwise direction calculating the sign of the $y$-component of each vertex in the sorted list until the sign changes. If the sign from step 5 is negative, one uses the same process, but moves in a counter-clockwise fashion.

6. The edge in which the intersection occurs is defined by a change in sign of the $y$ component of the vertices as one steps through the sorted list. The control surfaces associated with this edge are identified from the corresponding locations within the sorted list of rotation angles, $\phi_{j}$.

Once the general procedure for solving the two-dimensional optimal allocation problem has been defined, one can make some definitive conclusions about the method. First, by inspect- 
ing Figure 4.4, one can infer that only one quadrant of vertices is required to determine the solution. In addition, further examination reveals that the change in $y$-component of each vertex can be calculated by subtracting the contribution of the previous vertex and adding the component of the next vertex. Furthermore, one does not necessarily need to use the actual values of $\phi_{j}, j=1 \ldots m$ to produce a sign change, just their relative magnitudes are required. With all of this in mind, one is now ready to move on to the three-dimensional problem.

\subsection{Three-Dimensional Problem}

In the three-dimensional problem one considers $\mathbf{B} \in \mathcal{R}^{3 \times m}$. As a parallel to the twodimensional problem discussion, the $\mathbf{B}$ matrix is comprised of three rows,

$$
\mathbf{B}=\left[\begin{array}{l}
\mathbf{r}_{1} \\
\mathbf{r}_{2} \\
\mathbf{r}_{3}
\end{array}\right]
$$

Shown in Figure 4.5 is the attainable moments/objectives for the F-15 ACTIVE at an arbitrary orientation for the flight condition of 400 knots and 10,000 ft with twelve controls. The colors in this figure and all other AMS plots are arbitrary. For this illustration, a flaps-up configuration was chosen. The $x, y$ and $z$ coordinates correspond to the three moments/objectives, $C_{l}, C_{m}$ and $C_{n}$, respectively.

Similar to the two-dimensional problem, it is assumed that the three-dimensional problem is transformed by a matrix $T_{x}$ that aligns the desired objective vector direction $\ell$ with the positive $x$-axis. Note that this transformation is not uniquely defined since rotation about the $x$-axis does not affect the alignment of $\ell$ with the $x$-axis. Despite this non-uniqueness of the transformation, $T_{x}$ is determined using direction cosines and is an orthogonal matrix whose determinant is mathematically forced to be +1 . The actual calculation of $T_{x}$ is not important, only the fact that this transformation exists is of relevance.

Once transformed, we view the attainable moment subset from a vantage point aligned with the positive $z$-axis. That is, we view the AMS from a point of view that is perpendicular to the $x-y$ plane. This view is shown in Figure 4.6. 


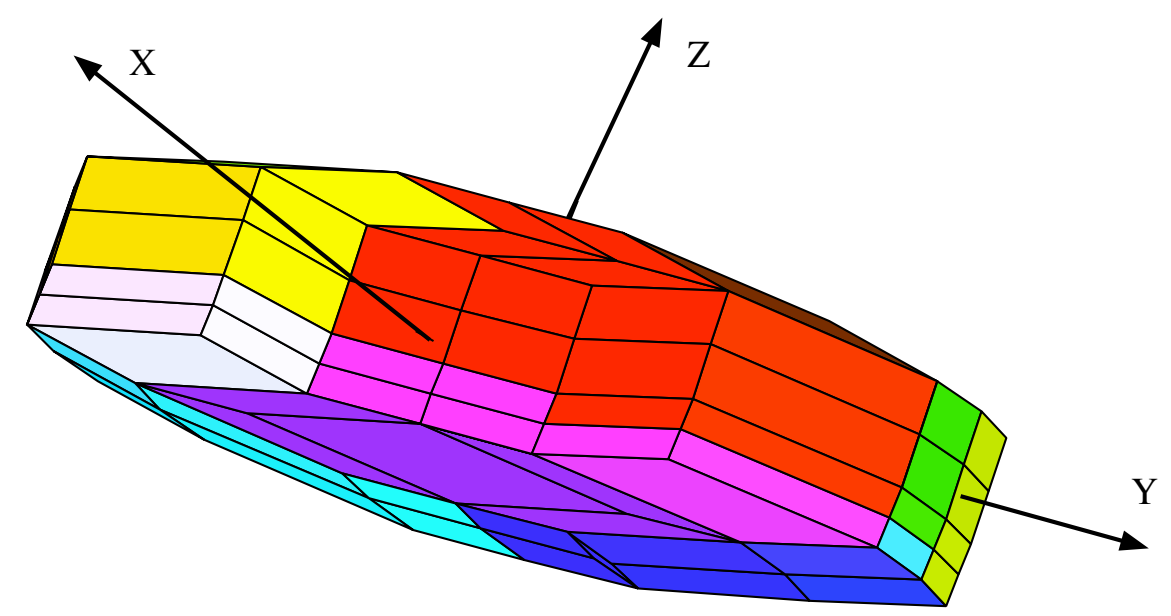

Figure 4.5: Three-Dimensional AMS for the F-15 ACTIVE at 400 knots and 10,000 ft with twelve controls. Arbitrary AMS orientation depicted.

Figure 4.6 is identical to Figure 4.1 with the addition of the facets corresponding to the third dimension drawn. Noting that the desired objective has been aligned with the $x$-axis, one can clearly see that the intersection of $\ell$ with the AMS occurs somewhere inside the purple facet. For reference, the point of intersection will be denoted $p$. Although it is visibly clear which facet defines the solution, one must be able to come to the same conclusion mathematically.

First, we view Figure 4.6 as a two-dimensional problem and identify the edge that crosses the $x$-axis. Keep in mind, this edge does not necessarily belong to the desired solution facet. Furthermore, if this edge did belong to the desired facet, a single edge is not sufficient to define a facet. If one now rotates the figure about the $x$-axis through a rotation matrix $T_{\theta}$, at some angle $\theta_{p}$ the point $p$ will lie exactly on the exterior of the hull projected into the $x-y$ plane, as viewed from the positive $z$-axis. In reality, there are two rotation angles, separated by $\pi$ radians, at which this situation occurs. Based on the geometry of the problem, in order to have the intersection point $p$ lie exactly on the exterior of the 3-d AMS projection into the $x-y$ plane, the plane that the intersection facet lies in must be perpendicular to the $x-y$ plane. This situation is depicted in Figure 4.7.

We assume that the angle $\theta_{p}$ can be found such that it satisfies the intersection criteria previously described. By rotating the figure through small angles, $\pm \Delta \theta_{p}$, about the angle $\theta_{p}$, the point $p$ will move frontwards and backwards in the $z$-direction from its position in the $x-y$ plane. Similarly, the two edges that define the facet that contains the intersection point 


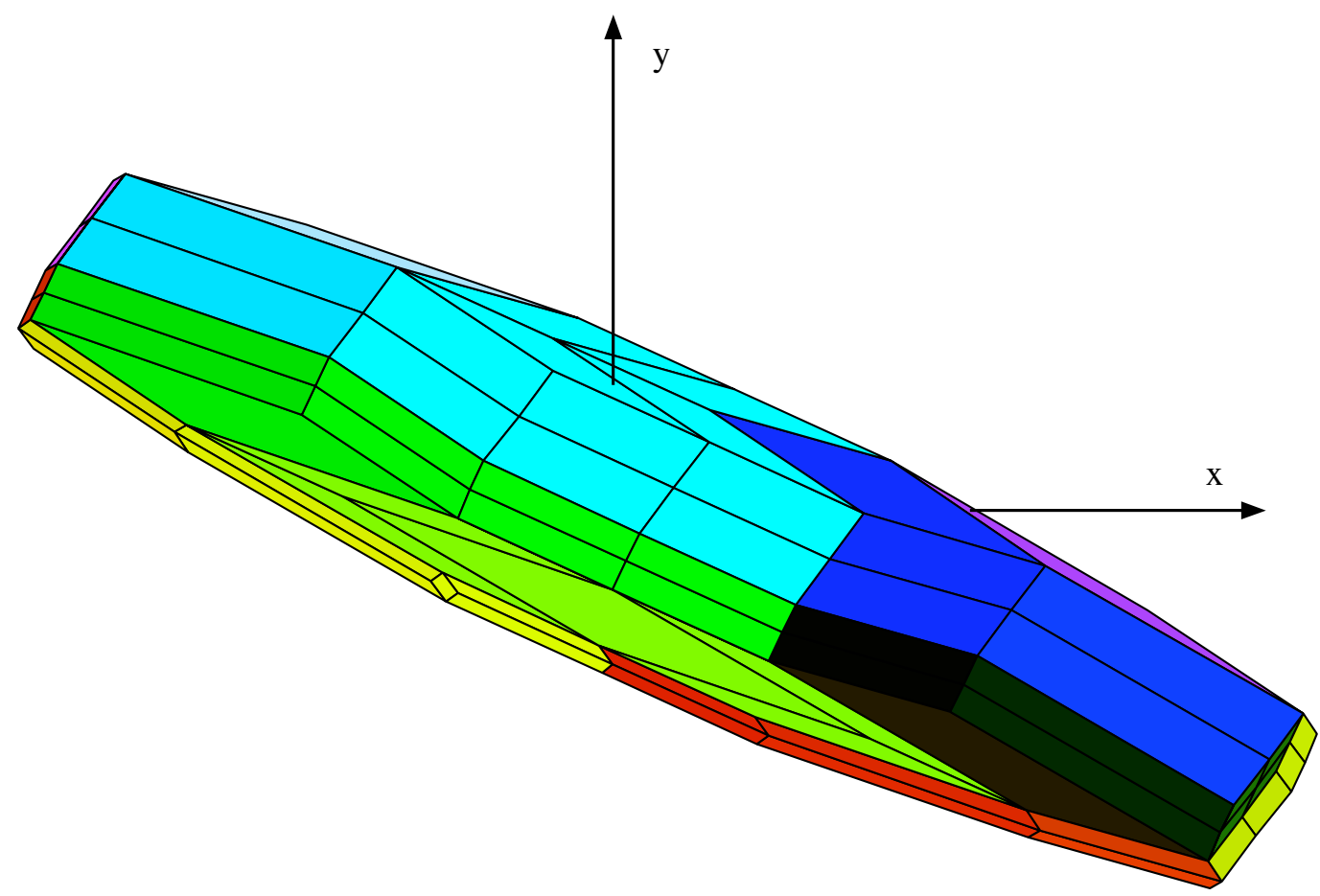

Figure 4.6: Three-Dimensional AMS for the F-15 ACTIVE at 400 knots and 10,000 ft with twelve controls. AMS oriented such that $\ell$ is aligned with the positive $x$-axis and viewed from the vantage point of the positive $z$-axis.

will alternately make up the edge of the two-dimensional projection, one being defined as "in front" of $p$, the other as "behind" $p$. The distinction between the two edges lies in their $z$-components. The edge that lies "in front" has a positive $z$-component while the "behind" edge has a negative $z$-component. Since these two edges will alternately lie on the hull of the two-dimensional projection, they may be identified utilizing the two-dimensional problem discussed in section 4.2 .

An analytical means of calculating the angle $\theta_{p}$ was not found in this research. However, the interest does not lie in the angle, $\theta_{p}$. Rather, one seeks the two edges that define the desired facet. (ie. the two facets that lie "in front" and "behind" the point $p$.) The process of determining these two edges is relatively straightforward. First, we begin with an arbitrary orientation of the AMS in which the half-line $\ell$ that defines the desired moment is coincident with the positive $x$-axis, as shown in Figure 4.6. We identify the edge that $\ell$ intersects utilizing the two-dimensional optimal allocation techniques from section 4.2. In addition, one must also calculate the $z$-component of this intersection. Next, rotate the entire AMS through a predefined angle $\theta_{0}$ about the $x$-axis. After the rotation, a new intersection edge 


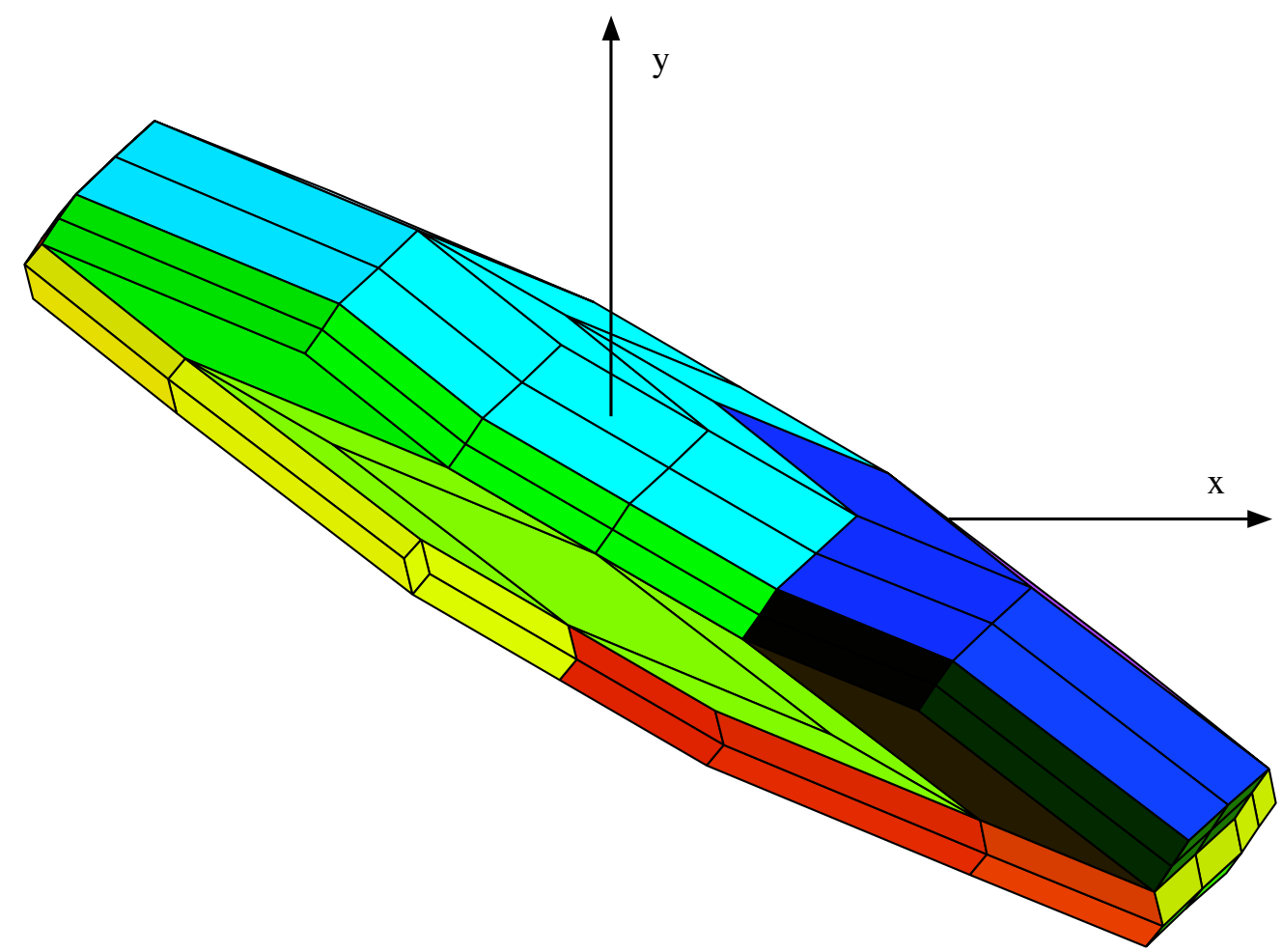

Figure 4.7: Three-Dimensional AMS for the F-15 ACTIVE at 400 knots and 10,000 ft with twelve controls. AMS oriented such that $\ell$ is aligned with the positive $x$-axis and viewed from the vantage point of the positive $z$-axis. AMS has been rotated about the $x$-axis to place the intersection point on the limb.

can be identified through the two-dimensional process. The $z$-component of this new edge is calculated and its sign is evaluated. This process is repeated until the sign of the $z$ component of the identified edge changes, at which time the last two edges identified are comprised of one edge that lies "in front" of the $x$-axis and one that lies "behind" it. These two edges are now the candidates for defining the desired facet. A check is performed to determine if the two identified edges define a facet. If they do define a facet, this facet will contain the intersection point $p$, and the solution is found. However, if these two edges do not define a facet, the direction of the rotation is reversed and a smaller angle $\theta_{1}$ is used. These steps are repeated until the desired facet is found. This entire process of solving the three-dimensional optimal allocation problem is summarized by the following list:

1. Orient the AMS such that the half-line $\ell$ is coincident with the positive $x$-axis and view the AMS from the vantage point of the positive $z$-axis. 
2. Use the techniques of the two-dimensional optimal allocation problem to determine the edge of the two-dimensional projection of the AMS that $\ell$ intersects.

3. Find the $z$-component of the intersection.

4. Rotate the entire AMS in three-dimensions about the $x$-axis through an angle $\theta_{i}$.

5. Repeat steps 2 and 3 for the edge that $\ell$ intersects after the rotation.

6. Check the sign of the $z$-component of the newly identified edge. If the sign has not changed from the previous edge, return to step 4. If the sign has changed, one of the edges now lies "in front" of the intersection point $p$ and the other lies "behind" it. Continue on to step 7.

7. Determine if the two identified edges comprise a facet.

8. If the edges do define a facet, the solution is found. If the edges do not belong to the same facet, reverse the direction of the rotation and reduce the size of the rotation angle, $\theta_{i}$. Repeat steps 4 through 7 until the desired facet is found or a set number of reductions in angle magnitude has been exceeded. If one exceeds a set number of reductions, the solution can be estimated utilizing the last two distinct edges that were found.

An initial rotation angle of $\theta_{0}=\pi / 4$ was chosen for the implementation used in this research. The subsequent angles, $\theta_{i}, i=1 \ldots N_{\text {bisections }}$, were calculated by using a bisection at each rotation reversal. To eliminate the repeated calculation of the sines and cosines of the rotation angles for the transformations, a table of sines and cosines of $\pi / 4, \pi / 8, \pi / 16, \ldots$ was pre-calculated. The maximum number of bisections allowed, $N_{\text {bisections }}$, was set to 10 for all data taken in this research. If 10 bisections did not produce an answer, an approximate answer was calculated using a linear interpolation of the $y$ and $z$-components of the vertices that defined the last two distinct edges found. The intersection point on $\ell$ was then used to combine the controls associated with the vertices used in the approximation to determine the solution. The last two edges found before entering the solution estimator in general were distinct, however, implementation found that there was a possibility of the same edge being found twice in a row. In this case, one of the repeated edges was utilized as well as the edge found prior to the pair of repeated edges. The errors associated with this estimation are discussed in section 4.4.3. 


\subsection{Timing Analysis}

The computational efficiency of the Bisecting, Edge-Searching Algorithm (BESA) is compared to that of the original brute-force facet-searching method as well as two other allocation methods. The data presented in this section is taken with permission from reference 3 by Durham. Durham's results are not duplicated because his work shows conclusive evidence of the computational efficiency of the BESA. In reference 3, Durham investigates the number of floating point operations involved with each of the control allocation algorithms. All evaluations are done utilizing a Matlab version of the allocation algorithms with randomly generated control effectiveness matrices and desired objectives. All control effector position limits are set to \pm 1 . Each trial of data consists of 100 different problems and their associated statistics. The maximum and mean number of floating point operations required was calculated for each of the algorithms. In the case of the Bisecting, Edge-Searching Algorithm, if a solution was not found within the desired number of bisections (set to 5) then an estimate of the solution was made. Comparing this solution to that attained utilizing the brute-force facet-searching method allows one to numerically compute the level of accuracy that the estimated solution achieved.

\subsubsection{Allocation Methods Investigated}

In addition to the Bisecting, Edge-Searching Algorithm and the brute-force facet-searching algorithm, a pseudo-inverse solution as well as a cascaded generalized inverse (CGI) solution were investigated. The number of floating point operations required for the brute-force facetsearching algorithm was determined assuming the worst case (ie. the last facet examined contained the solution). The pseudo-inverse solution was calculated utilizing $P=B^{T}\left[B B^{T}\right]^{-1}$ and the Matlab inverse function. In order to ensure that the determined solution was admissible, the solution was scaled after it was calculated. Past results have shown that the pseudo-inverse solution often produces inadmissible solutions. [19] Although the pseudoinverse is not very computationally intensive, it is included in Durham's comparison to show the trade-off between reducing the level of complexity and attaining sub-optimal results.

The final allocation method implemented was the cascaded generalized inverse. The CGI begins as the pseudo-inverse solution. However, if a saturation of controls occurs, those saturated controls are set at their corresponding position limits and then removed from the 
problem. The contribution of these controls being at their stops is subtracted from the desired objective. The problem is then resolved utilizing the remaining control surfaces, the new desired objective and the appropriate columns of the $\mathbf{B}$ matrix. This process is repeated until the remaining controls are either unsaturated or number fewer than three. In the case that the number of controls falls below three, Durham implemented a least squares solution for the remaining objective utilizing the last two controls.

\subsubsection{Required Floating Point Operations}

The number of required floating point operations are plotted in Figure 4.8 for the brute-force facet-searching algorithm. Only the cases of four to twenty controls are evaluated since the computational complexity was far too daunting above twenty controls.

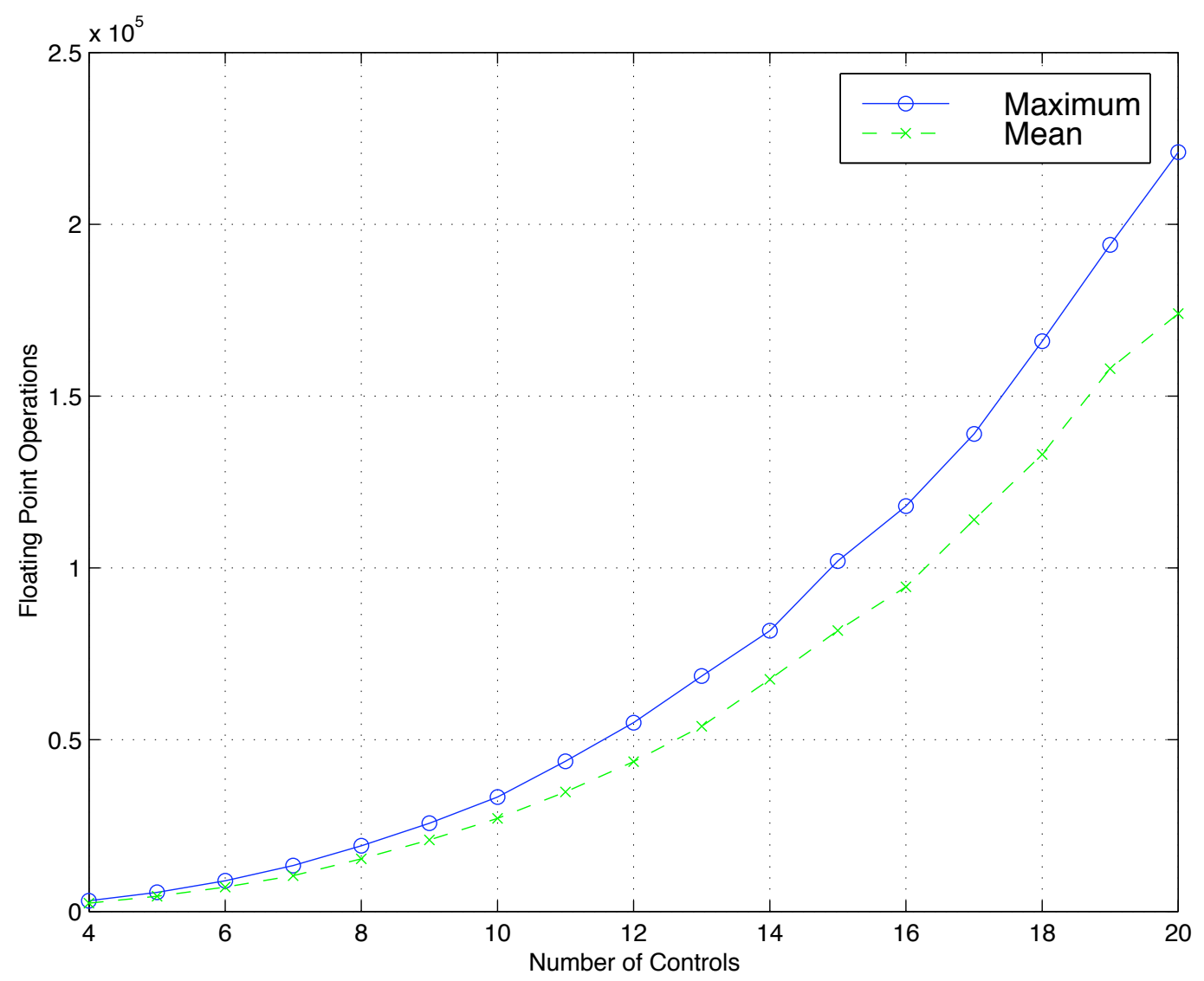

Figure 4.8: Required number of floating point operations for brute-force facet-search.

In reference 3, Durham performed a quadratic polynomial curve fit utilizing least-squares 
errors for the maximum and mean number of floating point operations required for the worst case scenario of the brute-force facet-searching method. The worst case is defined as having to search each pair of facets for the solution. His results, both yielding an error of $r^{2}=0.999$, are given in equations 4.7 and 4.8 for the maximum and mean number of floating point operations, respectively. In these equations, $m$ represents the number of control effectors.

$$
\begin{aligned}
\text { fflops }_{\text {Max }} & =6968-1244(m-4)+901(m-4)^{2} \\
\text { fflops }_{\text {Mean }} & =5122-807(m-4)+712(m-4)^{2}
\end{aligned}
$$

Timing results are shown in Figure 4.9 for the other methods evaluated, including the Bisecting, Edge-Searching Algorithm with a maximum of five bisections used. Results are shown for four to twenty controls. Results incorporating up to 500 control effectors can be found in reference 3 .

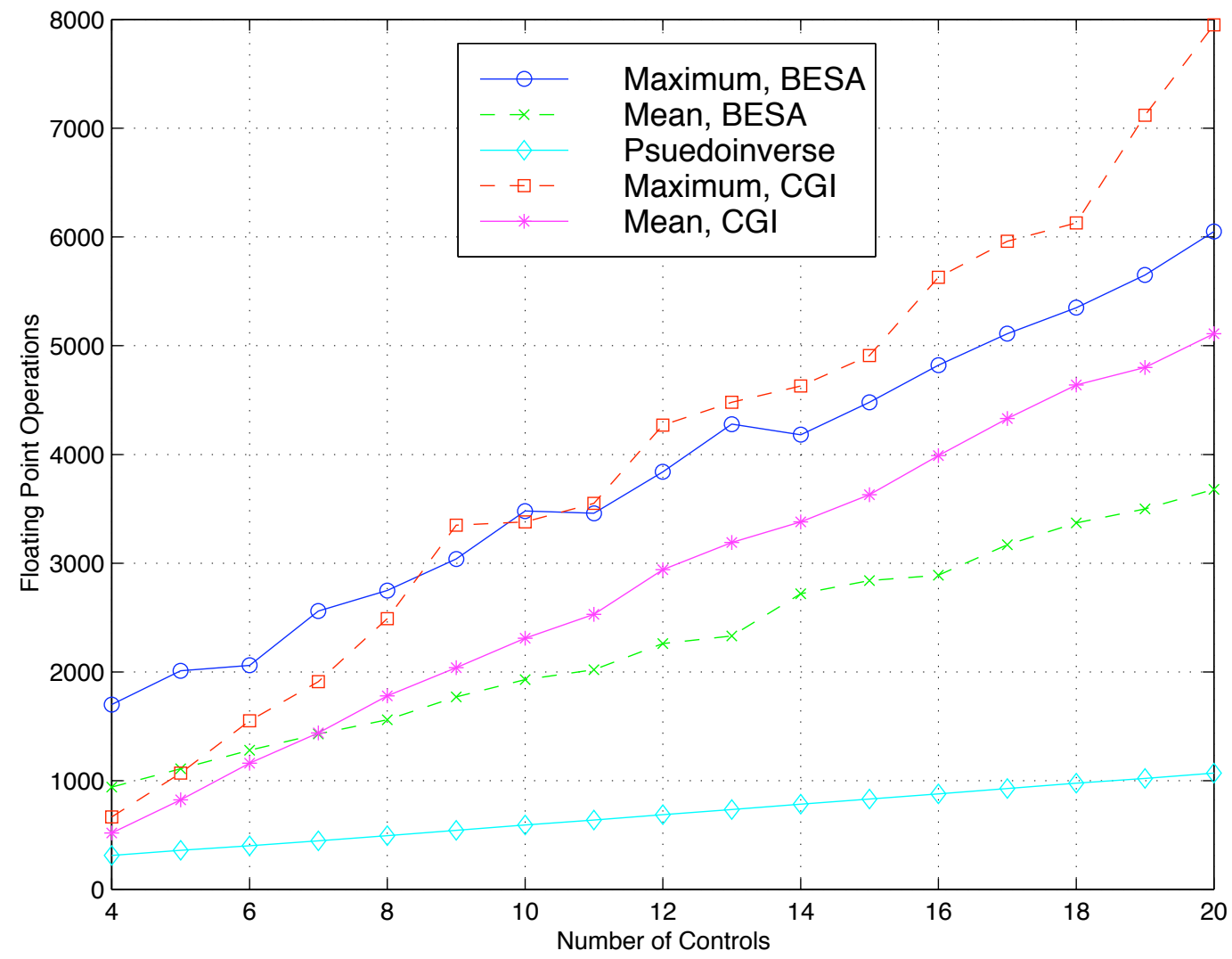

Figure 4.9: Required number of floating point operations, other methods.

Linear relationships were determined for each of the methods investigated. Durham found 
that the pseudo-inverse data fit perfectly with,

$$
\text { nflops }_{\text {Pseudo }}=307+48(m-4)
$$

The maximum and mean number of floating point operations required for the BESA algorithms where fit with the following curves.

$$
\begin{aligned}
\text { flops }_{\text {BESA }} A_{\text {Max }} & =1701+264(m-4) \\
n f l o p s_{B E S A_{\text {Mean }}} & =904+172(m-4)
\end{aligned}
$$

The corresponding linear regressions were $r^{2}=0.994$ for the maximum and $r^{2}=0.996$ for the mean. The data for the CGI was fit with straight lines for the maximum and mean number of flops. Regressions of $r^{2}=0.999$ were found for the curve fits of both the maximum and mean CGI data. The resulting relationships are,

$$
\begin{aligned}
\text { flops }_{C G I_{\text {Max }}} & =746+414(m-4) \\
n \text { flops }_{C G I_{\text {Mean }}} & =580+285(m-4)
\end{aligned}
$$

From Figure 4.9 one can determine that after approximately ten or eleven control effectors, the maximum number of floating point operations required for the Bisecting, Edge-Searching Algorithm falls below that required for the CGI. The slope of the worst case BESA algorithm curve was found to be close to 5.5 times that of the pseudo-inverse solution. However, a tradeoff is made between solution optimality and computational efficiency with the pseudo-inverse method. The reader is referred to the discussion in reference 3 regarding the high percentage of error associated with the scaled pseudo-inverse solution.

\subsubsection{Error Analysis}

As stated above, in this timing analysis, the maximum number of bisections was limited to five. After five bisections if a solution has not been determined with the BESA method, an estimate of the solution was made utilizing the two previous edges found. For the cases of four to twenty controls the estimated solution was compared directly to the optimal solution obtained from the brute-force facet-searching algorithm. The number of errors found using the BESA for a series of 100 problems is plotted in Figure 4.10 as a function of number 
of control effectors. From Figure 4.10 one can clearly see a trend of increasing number of errors as number of controls increases. This is to be expected since increasing the number of controls in the problem adds more computational complexity, (ie. more flops), as was shown in Figure 4.9.

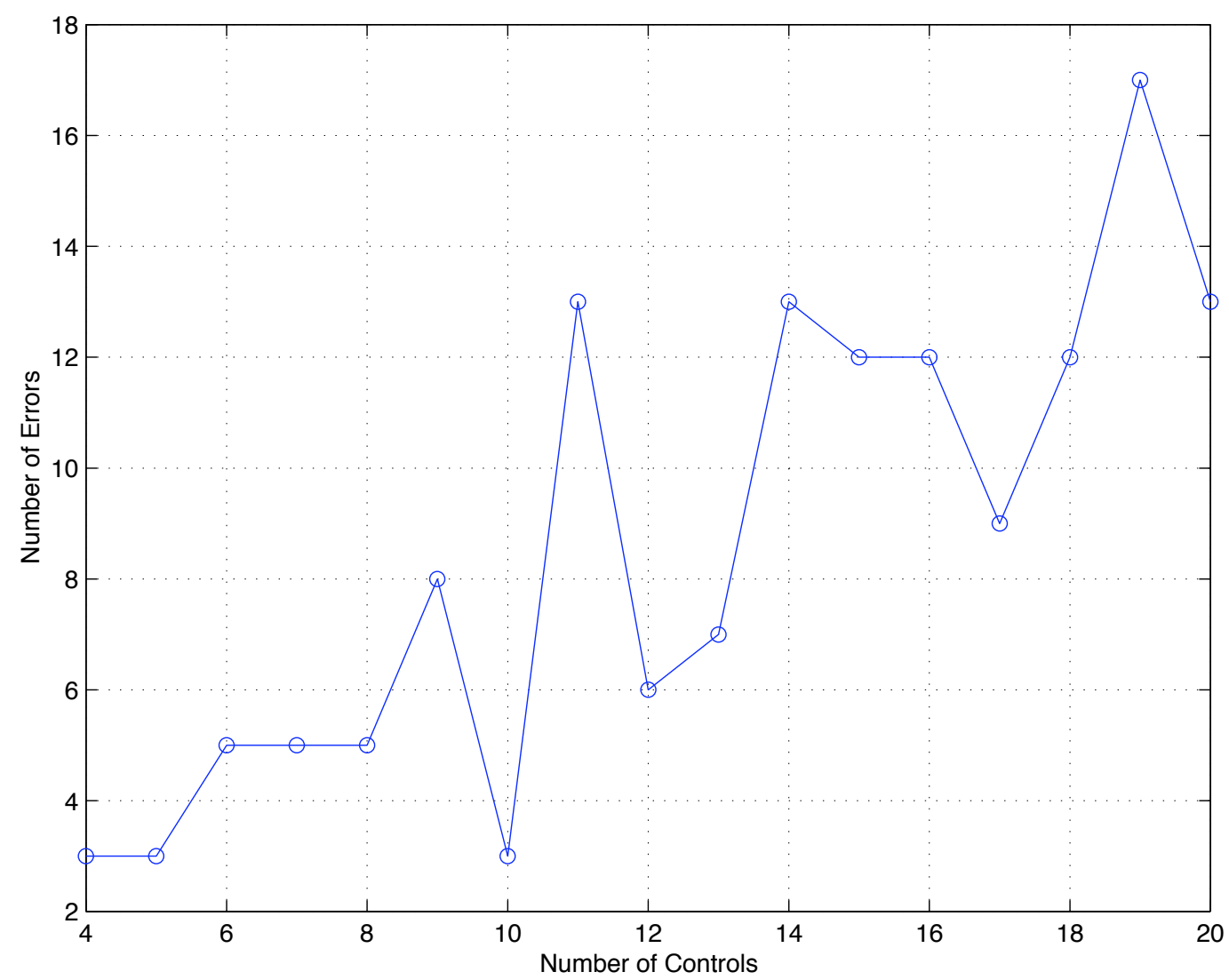

Figure 4.10: Number of errors using Edge-Searching Algorithm.

However, Durham found that as the number of controls increased, the percentage error associated with these errors generally declined. In fact, results taken from Durham and shown in Figure 4.11 indicated for the 100 problems investigated, the maximum error is $4.4 \%$ at the four controls point and dwindled to mere $0.034 \%$ for the case of eighteen controls. It was determined that as the number of control effectors increased, the angle that each facet of the AMS subtended decreased and therefore the use of a nearby incorrect facet resulted in a solution that was "close" to the actual value. Or conversely, with a small number of control effectors, the facets subtend larger angles and the utilization of an incorrect facet in the solution vector introduces a larger error since the erroneous facet is "far" from the the actual solution. Simply stated, with a large number of controls two erroneous edges will be 
closer together than two erroneous edges of an AMS with a small number of controls.

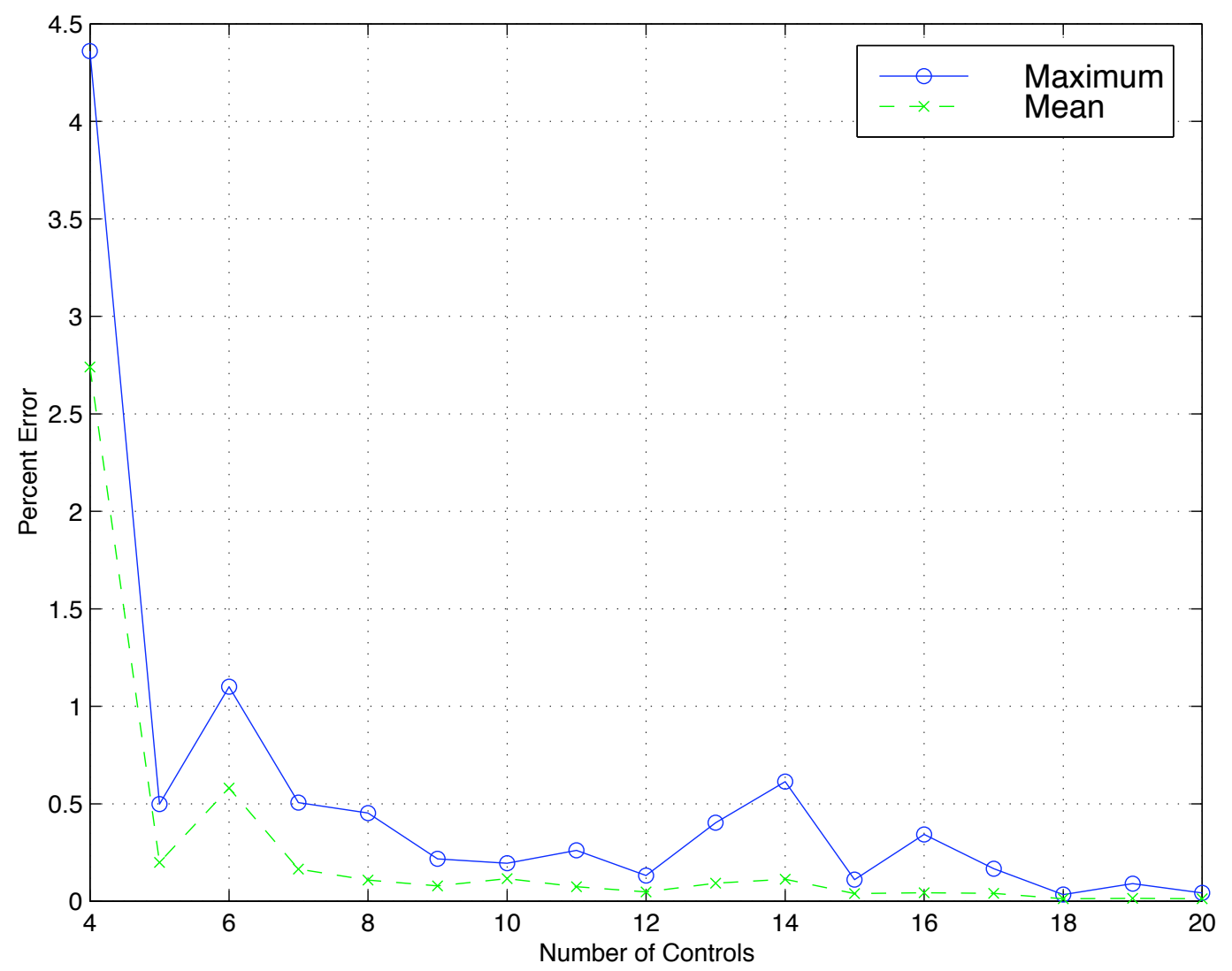

Figure 4.11: Percentage error using Edge-Searching Algorithm.

\subsubsection{Timing Conclusions}

The previous subsections have presented data that supports the claim that the Bisecting, Edge-Searching Algorithm is a computationally efficient replacement for the brute-force facet searching portion of Moment Rate Allocation. In addition, it was shown that although a solution is not always attained within the desired number of bisections, an estimate of the solution can be substituted in these cases that is very accurate. In fact, as was shown in Figure 4.11, as the problem becomes more complex, (ie. more control effectors are included), the percentage error associated with the estimated solution rapidly declines.

Evaluation of the number of floating point operations required per control effector for each of the allocation methods investigated in Durham's work revealed linear relationships for 
the BESA as well as the pseudo-inverse and CGI methods and a quadratic relationship for the brute-force facet-searching method. The linear relationship for the BESA algorithm is deemed a substantial savings in computational complexity over the previously used brute-force facet-searching algorithm, especially as the number of control effectors present on modern tactical aircraft continues to rise. However, based on computational complexity, one should question the choice of the BESA algorithm over the pseudo-inverse solution or the CGI solution. Figure 4.9 did show that the pseudo-inverse solution is far more efficient than the BESA algorithm. In fact, up to about ten or eleven controls the CGI is more efficient than the BESA method. In response to these questions, the reader is referred to Durham's work. In reference 3, Durham evaluated the optimality of the solutions attained by the pseudo-inverse and the CGI. Durham looked at the percentage error associated with the solutions found using the pseudo-inverse and the CGI as compared to those attained with the facet and BESA methods. Although no data is presented here, Durham's work offered conclusive evidence that in terms of optimality of the control allocation solution, the BESA algorithm was superior to the pseudo-inverse and CGI solutions. [3] 


\section{Chapter 5}

\section{Restoring Methods}

\section{$5.1 \quad$ Background}

Frame-wise control allocation as implemented in this research was designed to account for control effector position and rate constraints. Yet nothing in its design ensures that the controls will end up in any "desirable" configuration after attaining the commanded objective. This potential for "undesired" control configurations is a direct result of Moment Rate Allocation's method of calculating a control effector's position. Starting with a desired moment vector, $\mathbf{m}_{\mathbf{d}}$, an initial control configuration, $\mathbf{u}_{0}$, is determined. However, after $k$ time steps, the control positions will be located in some configuration determined by $\mathbf{u}_{k}$,

$$
\mathbf{u}_{k}=\mathbf{u}_{0}+\sum_{i=1}^{k} \Delta \mathbf{u}_{i}
$$

From equation 5.1, one can see that the position $\mathbf{u}_{k}$ depends upon the summation of $\Delta \mathbf{u}_{i}$ from each of the previous iterations. Thus the current position is dependent on each of the previous positions. This means that when a pilot returns the control stick to its center position after the completion of a maneuver, the controls will not necessarily return to their "zero" positions. The further objective of driving the controls towards a "desired" configuration $\mathbf{u}_{d}$ can be achieved in control allocation by incorporating restoring methods. These desired control effector configurations have the potential to minimize the drag on the aircraft or simply drive the controls back to some predetermined "zero" location. However, the modified control configuration must not only lie in the subset of admissible controls, but 
must also still be capable of obtaining the original objective, the desired moments.

The following sections will discuss the implementation of several different forms of control restoring. It should be noted that the emphasis in control restoring is to attain a fourth objective while still achieving the original three desired moments. However, as will be shown in section 5.4, control allocation and control restoring do not always produce desired forces on the aircraft. This introduction of undesired forces on the aircraft follows directly from the assumption that the primary function of control effectors on an aircraft is to generate the moments, roll, pitch and yaw. The validity of this assumption is questioned when one considers restoring methods such as maximum-norm restoring. It will be shown that as controls begin to migrate away from their zero positions, as is commanded in maximum-norm restoring (anti-restoring), unwanted large forces can develop on the aircraft. In particular, excessive sideforce on the aircraft occurs. Section 5.4 will discuss the minimization of this sideforce. Although not thoroughly discussed in this paper, maximum-norm restoring was used by Leedy [1] in an attempt to drive the controls towards their limits to examine the potential of control wind-up during a maneuver. Leedy showed that control wind-up, now referred to as Null Space Saturation (NSS), did not have a crippling effect on the aircraft performance as was initially feared.

In general, the additional fourth criterion's solution is based on the solution to what is defined as the global allocation problem. Considering the global allocation problem, one seeks the combination of control effectors, $\mathbf{u}_{d}$, that solves

$$
\mathbf{y}_{d}=\mathbf{B}_{\text {global }} \mathbf{u}_{d}
$$

The control effectiveness matrix $\mathbf{B}_{\text {global }}$ is usually defined as the Jacobian matrix evaluated about a zero control deflection. Therefore, $\mathbf{B}_{\text {global }}$ can be represented as

$$
\mathbf{B}_{\text {global }}=\left[\frac{\partial \mathbf{y}}{\partial \mathbf{u}}\right]_{\mathbf{u}=0}
$$

Using rate-based allocation methods, the restoring solution will generate a delta to the controls, $\Delta \mathbf{u}_{\text {rest }}$. Once $\Delta \mathbf{u}_{\text {rest }}$ has been determined, the solution to the control allocation problem becomes

$$
\mathbf{u}_{k}=\mathbf{u}_{k-1}+\Delta \mathbf{u}_{k}+\Delta \mathbf{u}_{r e s t, k}
$$


The focus of the remainder of this chapter is the determination of $\Delta \mathbf{u}_{r e s t}$ for different restoring criterion. Although the possibilities for restoring criterion are unlimited, only variations of minimum-norm and minimum-sideforce restoring are investigated in this work.

\subsection{Restoring to a Known Solution}

In some instances, the desired restoring criterion is a known configuration that can be described mathematically. There are, in general, an infinite number of control combinations $\mathbf{u}$ that satisfy the equation $\mathbf{y}=\mathbf{B u}$. Any two solutions, $\mathbf{u}_{1}$ and $\mathbf{u}_{2}$, differ by a vector $\mathbf{u}^{\perp}$ that lies in the null space of $\mathbf{B}$. By inspection, it can be shown that $\mathbf{u}^{\perp}=\{\mathbf{u} \mid \mathbf{B u}=0, \mathbf{u} \neq 0\}$. As proof, consider

$$
\mathbf{B}\left(\mathbf{u}_{2}-\mathbf{u}_{1}\right)=\left(\mathbf{B u}_{2}-\mathbf{B} \mathbf{u}_{1}\right)=(\mathbf{y}-\mathbf{y})=0
$$

From equation 5.5, it is clear that $\left(\mathbf{u}_{2}-\mathbf{u}_{1}\right) \in \mathbf{u}^{\perp}$. The implementation of control restoring to a known solution involves first determining $\left(\mathbf{u}_{k-1}+\Delta \mathbf{u}_{k}\right)$ that solves the primary objective $\mathbf{y}_{d_{k}}$, and then augmenting $\left(\mathbf{u}_{k-1}+\Delta \mathbf{u}_{k}\right)$ by the additional restoring contribution $\mathbf{u}_{k}^{\perp}$ that drives the controls towards satisfying the additional criterion.

In previous work by Leedy and Durham [1], the rate-based allocation utilized a localized control effectiveness matrix $\mathbf{B}_{k}$ to determine $\Delta \mathbf{u}_{k}$. The use of a local control effectiveness matrix for allocation created a problem if one attempted to use the global control effectiveness matrix, $\mathbf{B}_{\text {global }}$, to generate a restoring vector, $\mathbf{u}_{k}^{\perp}$. The origin of the problem was that the use of $\mathbf{B}_{\text {global }}$ in the determination of $\mathbf{u}_{k}^{\perp}$ could have an influence on the validity of the primary objectives.(ie. $\mathbf{B}_{\text {global }} \mathbf{u} \neq \mathbf{B}_{k} \mathbf{u}$ ) In their work, Leedy and Durham concluded that in order to ensure that the primary objectives would remain satisfied during the restoring process, the restoring algorithms would also have to use the local effectiveness data. This problem was eliminated in the allocation method implemented in this work because global effectiveness data was utilized in the determination of $\Delta \mathbf{u}_{k}$ as well as $\mathbf{u}_{k}^{\perp}$.

Once $\mathbf{u}_{k}^{\perp}$ has been calculated, the solution vector to the control allocation problem becomes

$$
\mathbf{u}_{k}=\mathbf{u}_{r e s t, k}=\mathbf{u}_{k-1}+\Delta \mathbf{u}_{k}+C_{s} \mathbf{u}_{k}^{\perp}
$$

In order to ensure that the change in controls remains admissible, the scale factor $C_{s}$ has 
been included. $C_{s}$ is a scalar value that is calculated to satisfy

$$
\Delta \mathbf{u}_{r e s t, k}=\left(\Delta \mathbf{u}_{k}+C_{s} \mathbf{u}_{k}^{\perp}\right) \in \omega
$$

where $\omega$ is the more restrictive of the global position and rate limits for the current frame. A single scalar value of $C_{s}$ is used for the entire control vector $\mathbf{u}_{k}^{\perp}$ based on the most restrictive of the constraints on each individual control effector. In this work, a pseudo-inverse solution is used to generate $\mathbf{u}_{k}^{\perp}$ for the minimum-norm restoring.

\subsubsection{Minimum-Norm Restoring: Known Solution}

One type of restoring utilized in this research is minimum-norm restoring. The idea behind minimum-norm restoring is to use the remaining rate capabilities of the control effectors after they have achieved the desired moment to minimize the Euclidean 2-norm of the control effector positions. For the case in which the minimum-norm solution can be calculated, the controls can be driven towards this known solution. The right pseudo-inverse produces the inverse of the control effectiveness matrix that has the minimum Euclidean 2-norm. The right pseudo-inverse is defined in equation 5.8,

$$
\mathbf{P}=\mathbf{B}^{T}\left(\mathbf{B B}^{T}\right)^{-1}
$$

One first calculates the solution that satisfies the original desired objective, $\mathbf{y}_{\mathbf{d}}$, using the control effectiveness matrix, $\mathbf{B}_{k}$. The result is $\mathbf{u}_{1}=\left(\mathbf{u}_{k-1}+\Delta \mathbf{u}_{k}\right)$. The secondary criterion for the pseudo-inverse restoring is given by $\min \frac{1}{2} \mathbf{u}^{T} \mathbf{u}$. Using the pseudo-inverse matrix, a second valid solution for the desired objective is found to be

$$
\mathbf{u}_{2}=\mathbf{u}_{\text {pseudo, }, k}=\mathbf{P}_{k} \mathbf{y}_{k}
$$

The difference between these two solutions, $\mathbf{u}_{1}$ and $\mathbf{u}_{2}$, will lie in the null space of the $\mathbf{B}$ matrix. The resulting solution for the restoring vector, $\mathbf{u}_{k}^{\perp}$, is

$$
\mathbf{u}_{k}^{\perp}=\mathbf{u}_{\text {restored }}=\mathbf{u}_{1}-\mathbf{u}_{2}=\left(\mathbf{u}_{k-1}+\Delta \mathbf{u}_{k}-\mathbf{u}_{\text {pseudo }, k}\right)
$$

Thus, the vector $\mathbf{u}_{k}^{\perp}$ that is inserted into equation 5.6 to produce the total control solution, $\mathbf{u}_{k}$, has been found for the minimum-norm restoring to a known solution configuration. 


\subsection{Restoring to an Unknown Solution}

When the desired additional restoring criterion is not explicitly known, one can still restore in the direction of the desired criterion. This method involves the augmentation of the primary objective vector, $\mathbf{y}_{d}$, by an additional parameter, $y_{n+1}$, that is related to the additional criterion. Utilizing frame-wise control allocation, one attempts to satisfy the additional criterion in increments, $\Delta y_{n+1}$. The control effectiveness matrix is also augmented with a fourth row that corresponds to the control surfaces' effects on the additional criterion. The augmented objective vector takes the form

$$
\Delta \mathbf{y}_{\text {aug }}=\left\{\begin{array}{c}
\Delta \mathbf{y} \\
\Delta y_{n+1}
\end{array}\right\}
$$

where the primary objective $\Delta \mathbf{y}$ takes precedence over the secondary criterion $\Delta y_{n+1}$. In short, satisfaction of the secondary criterion can only be attempted with whatever control effector rate and/or position capabilities remain after allocating for $\Delta \mathbf{y}$. The augmented $\mathbf{B}$ matrix at the $k^{\text {th }}$ frame is defined as

$$
\mathbf{B}_{a u g, k}=\left[\begin{array}{c}
\mathbf{B}_{k} \\
\partial y_{n+1} / \partial \mathbf{u}
\end{array}\right]_{\mathbf{u}_{k}}
$$

Therefore, the solution to the primary objective is first calculated yielding $\Delta \mathbf{u}_{k}$ such that $\Delta \mathbf{y}_{d_{k}}=\mathbf{B}_{k} \Delta \mathbf{u}_{k}$. At this point, the origin of the problem is shifted to the location of $\Delta \mathbf{y}_{d_{k}}$ so that one can then solve for $\Delta \mathbf{u}_{k}^{\perp}$. The origin shift sets the primary objective to zero for the restoring portion of the solution and ensures that the primary objective is maintained during the attempt to satisfy the secondary criterion. In other words, the origin shift forces

$\Delta \mathbf{u}_{k}^{\perp}$ to lie in the null space of $\mathbf{B}_{a u g, k}$. Thus, the restoring problem becomes, find $\Delta \mathbf{u}_{k}^{\perp}$ such that

$$
\mathbf{B}_{a u g, k} \Delta \mathbf{u}_{k}^{\perp}=\left\{\begin{array}{c}
\Delta \mathbf{y}_{k}=0 \\
C_{s}
\end{array}\right\}
$$

The scaling factor $C_{s}$ is defined as a negative scalar specified to move toward the minimum of the additional vector $y_{n+1}$ while still ensuring that the controls solution falls within $\omega$, the more restrictive of the global position and rate limits for the given frame. 


\subsection{Minimum-Sideforce Restoring}

Minimum-sideforce restoring is classified as restoring to an unknown solution. The idea to attempt minimum-sideforce restoring arose from an observed excessive level of sideforce generated in a maximum-norm restoring configuration. Admittedly, maximum-norm restoring has no real-life application beyond its research role of driving the controls away from their Euclidian minimum-norm configuration. However, a high level of sideforce was observed with other restoring methods, including the case of no restoring. Figure 5.1 shows the roll-rate and associated sideforce for several restoring methods during a 3 inch lateral stick doublet at a flight condition of 400 knots and 10,000 ft. Minimum-sideforce restoring parallels in theory and implementation the minimum-drag restoring discussed in reference [18].

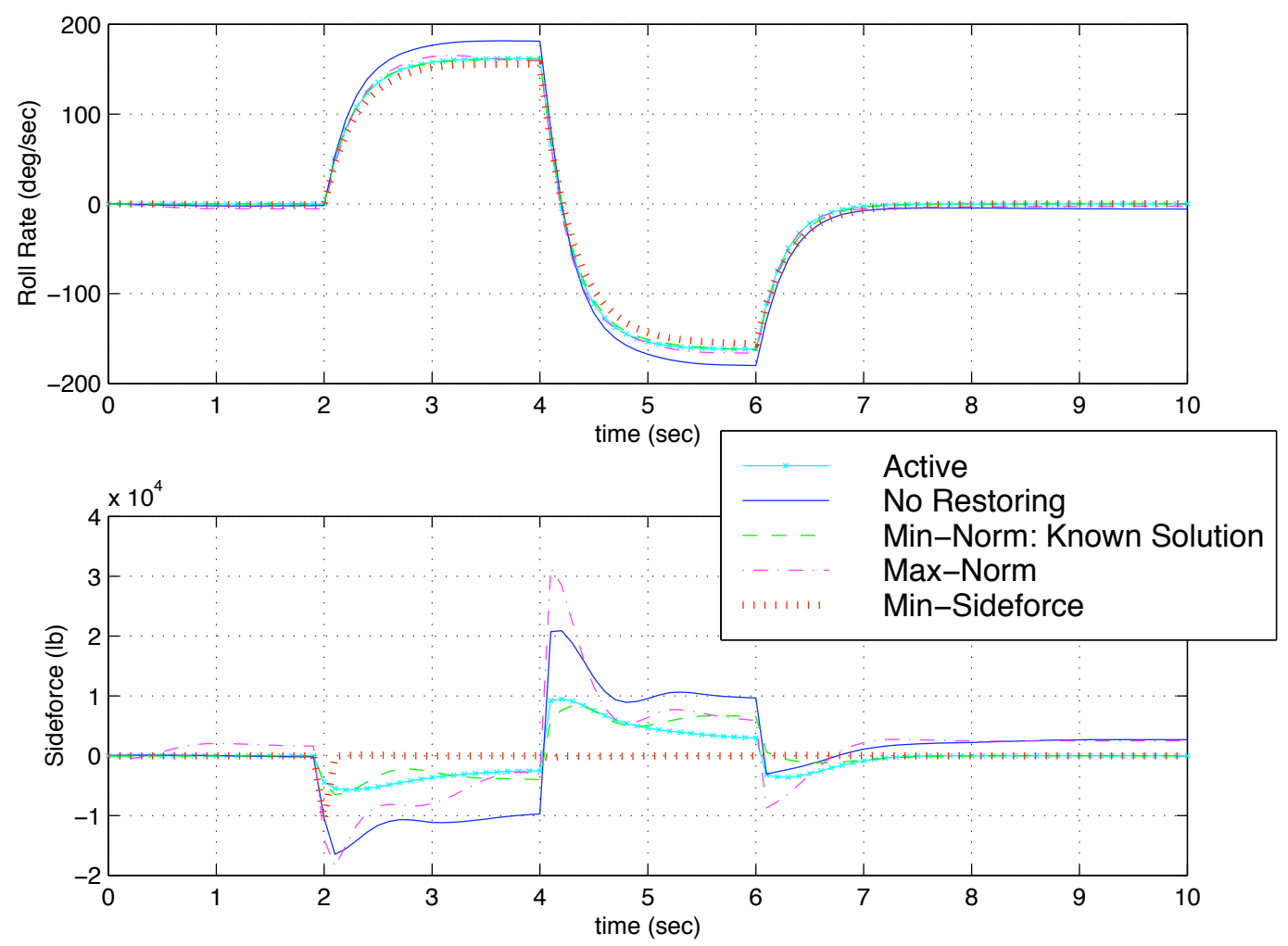

Figure 5.1: Roll rate and sideforce generated during a 3 inch lateral stick doublet for the F-15 ACTIVE at 400 knots and 10,000 ft.

From Figure 5.1 it is clear that all methods evaluated produced approximately the same roll rate response to the lateral stick doublet. However, as shown in the second subplot of Figure 5.1, minimum-sideforce restoring has clearly outperformed the other methods in 
regards to the elimination of total sideforce during the maneuver. As expected from previous discussions, the maximum-norm restoring case produced the largest magnitude sideforces of all the methods. From Figure 5.1 it is evident that the implementation of the minimumsideforce restoring has a significant positive impact on the elimination of unwanted sideforces on the aircraft.

\subsubsection{Variable Step Size Restoring}

In the majority of the restoring to an unknown solution work performed in the past, a fixed scaling of the control restoring vector was implemented in order to reduce the phenomena known as control chatter. This undesired high frequency oscillation of the control surfaces is caused by an overstepping of the desired objective. The pseudo-inverse control solution produced by the restoring algorithms is multiplied by a constant scalar to reduce the size of the step that the algorithm takes towards the unknown desired objective. Although this reduction in step size can effectively be used to eliminate the unwanted chatter, taking a smaller step towards the solution results in a slower convergence to the solution.

In this research, the variable scale factor was based on the ratio of the total sideforce on the aircraft to the weight of the aircraft with a maximum scale factor of 1.0 implemented in order to ensure that the final control effector solution lies within $\omega$. This ratio was chosen because as the aircraft's sideforce became large the algorithm forced the restoring step size to its maximum value of 1.0. Smaller values of sideforce resulted in shorter steps with a lower limit on the scale factor chosen to be $1 e-6$.

Time histories of the sideforce, left canard deflection and restoring step size are shown in Figure 5.2 for the 3 inch lateral stick doublet previously discussed. Data for this figure were recorded at a rate of $100 \mathrm{~Hz}$. Different values of the scalar scale factor have been used to illustrate the tradeoff between reduction in control chatter and convergence time during minimum-sideforce restoring. Chatter is found in the plot of canard deflection for the 0.1 and 0.05 restoring step sizes. The 0.01 restoring step size essentially eliminates the control chatter, but sacrifices convergence speed. From the subplot of canard deflection and sideforce, one can decisively state that the use of a variable step size in restoring produces the solution with the best combination of chatter reduction and convergence time. Note the large spikes in step size for the variable step size restoring case at the 2,4 and 6 second marks. These times correspond exactly to the instance of the lateral stick inputs. The size of 
the step increases dramatically at these points to drive the sideforce back to zero as quickly as possible without producing control chatter.

Utilization of a variable step size offers not only the prevention of control surface chattering, but faster convergence of the solution. Essentially, when the controls are well away from the desired solution a large step is taken. As the solution is approached, the step is decreased in size to eliminate the possibility of control chatter. In restoring methods like minimumsideforce, one can consider a situation where the sideforce is close to zero as satisfactory. Since a solution that is only close to zero is sought, one can approach the solution asymptotically without the concern that the exact solution will never be physically realizable.

Example uses of minimum-sideforce restoring can be found in the chapters that follow. Pilot comments about flights flown in the minimum-sideforce restoring configuration consistently indicate a positive attitude towards the reduction in sideforce on the aircraft. Furthermore, the results presented in Figure 5.2 clearly indicate a need to move towards the utilization of a variable step size restoring in order to achieve the optimal combination of control chatter reduction and objective minimization convergence time. It is suggested that future work investigate a more sophisticated variable step size restoring algorithm that has foundations in optimization techniques. 

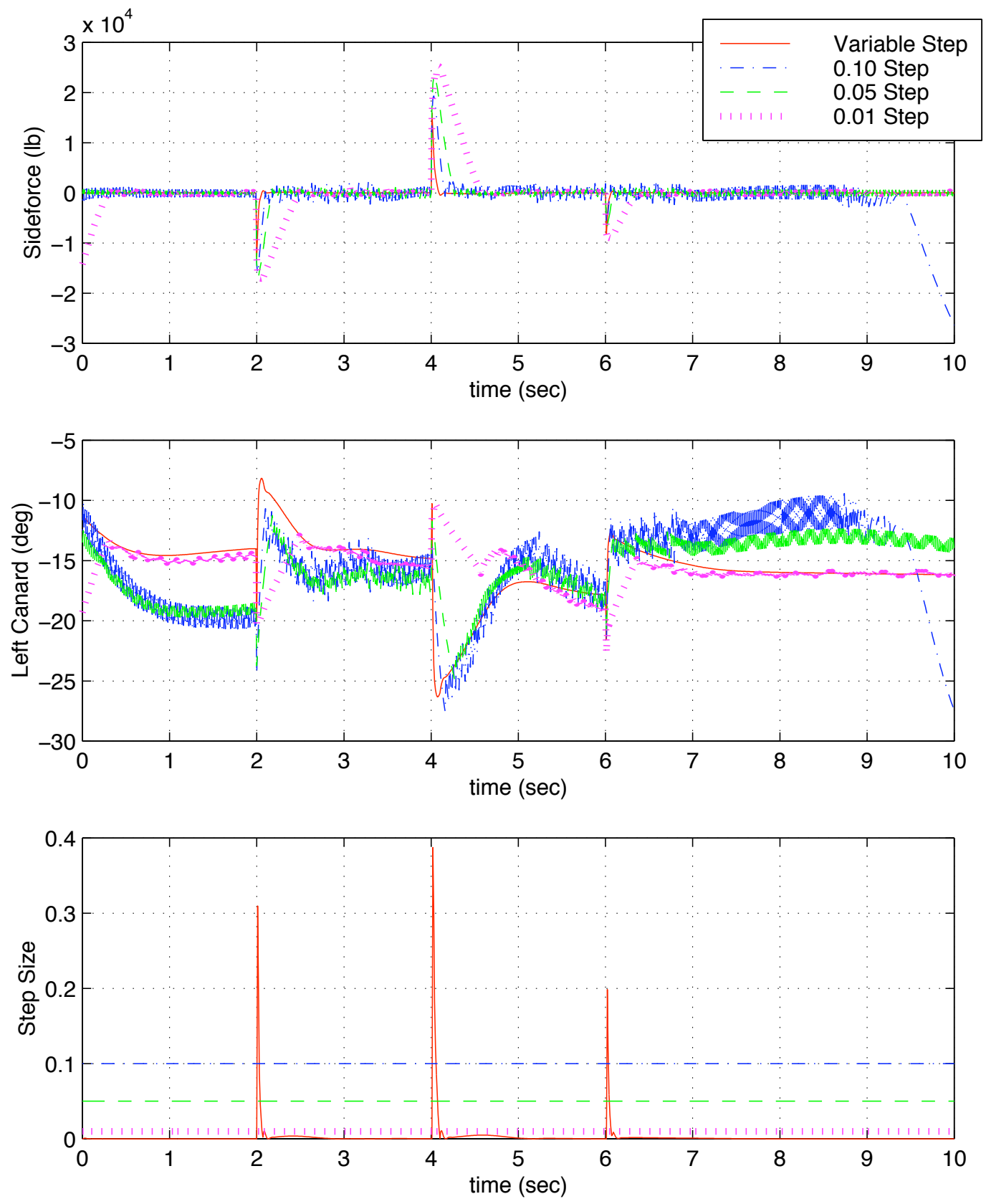

Figure 5.2: Time history of sideforce, left canard deflection and restoring step size during a 3 inch lateral stick doublet for the F-15 ACTIVE at 400 knots and 10,000 ft. 


\section{Chapter 6}

\section{Representative Maneuvers}

\subsection{Canned Input: Lateral Stick Doublet}

The use of canned or predetermined inputs to the control inceptors of an aircraft offers insight into a direct comparison of the aircraft responses for several control allocation or restoring methods. Since all methods are utilizing the same control inceptor inputs, the aircraft should respond in a similar manner for all cases investigated. The canned input examined in this portion of the research was a 4 inch lateral stick doublet at a flight condition of 400 knots and 10,000 ft. The doublet was initiated at a time of two seconds and each portion of the doublet was held for two seconds. This input was selected for evaluation because it appeared to exercise all the control effectors in the heart of the aircraft's flight envelope. Exact matching of aircraft state time histories was not achieved in this research. It is believed that this problem stems from an undefined control law - control allocator interaction. In addition, since the control law's interpretation of control inceptor input is a function of the aircraft state, if the state begins to differ at all between methods, then the commanded moments will differ. As a result of this difference, the aircraft response will further differ.

To eliminate the variance in aircraft response one might consider using an input command on the aircraft's desired moments. Although driving the desired moments would force the aircraft's responses to be identical for all combinations of restoring methods, this would effectively remove the control law from the problem. This removal of the control law and its interpretation of control inceptor inputs undermines the objective of this research to evaluate the control allocation algorithms on an aircraft with a high-fidelity control law that accounts 
for desired aircraft handling qualities.

Figures 6.1 through 6.5 show time histories of the aircraft response to the 4 inch lateral stick double. The red solid line indicates the response as calculated by the original control mixer that was present on the F-15 ACTIVE. The dashed green line represents the non-restoring version of the moment rate allocator. The blue dash-dot time histories correspond to a minimum-norm restoring solution and the cyan dotted lines depict the time history of the response utilizing a variable step size restoring minimum-sideforce restoring method.

As seen in the first and second subplots of Figure 6.1, the horizontal tails for the minimumnorm and ACTIVE solutions return to a symmetric configuration, after the completion of the maneuver. The minimum-sideforce and non-restoring solutions for the horizontal tails do not return to a symmetric configuration, but this non-symmetric deflection is to be expected since there is no additional objective driving them towards a symmetric configuration. The fact that the ACTIVE controls are returning to a symmetric configuration is a direct result of the control allocation method implementation. In the original ACTIVE control law, differential and symmetric commands are generated in response to control inceptor inputs. When the lateral stick is returned to its neutral position, the differential command disappears and thus the controls will return to a symmetric configuration. Again in the third and fourth subplots one can see that the ACTIVE and minimum-norm solutions for the left and right aileron return to a symmetric configuration while the minimum-sideforce and non-restoring solutions do not. Although the minimum-sideforce restoring algorithm returns the horizontal tails and ailerons to a symmetric configuration, zero control-generated sideforce does not require symmetric deflections, just equal and opposite left and right sideforces.

Figure 6.2 shows the time histories of the rudders and canards in response to the doublet. As seen in Figure 6.2, the minimum-sideforce and non-restoring versions of the allocator utilize differential rudders and canards to help generate the roll rate demanded in the maneuver. In addition, the canards and rudders, serving as the primary yaw generating control effectors, are used to regulate the aircraft's sideslip angle to zero. The minimum-norm restoring and ACTIVE solutions force the rudders to remain close to zero and utilize the canards primarily as pitch generators. The point about which the differential canards are used in the non-restoring and minimum-sideforce cases was closer to $-20^{\circ}$, while the other two cases operate about the $-4^{o}$ datum. As a result of the more aircraft nose down canard deflection in Figure 6.2, the minimum-sideforce restoring uses more positive pitch horizontal tail and aileron authority as can be seen in Figure 6.1. 
Time histories of pitch and yaw thrust vectoring during the maneuver are found in Figure 6.3. The minimum-norm and ACTIVE allocators do not use a large amount of yaw vectoring as is evident in the third and fourth subplots of the figure. As noted earlier, control effectiveness data could not be obtained for rolling or yawing moments due to pitch thrust vectoring, nor rolling or pitching moments due to yaw thrust vectoring. As a consequence of this lack of effectiveness data, the pitch and yaw thrust vectoring was in general used in a symmetric configuration, as is illustrated in Figure 6.3. Non-symmetric thrust vectoring was occasionally present. These differential commands were most likely a consequence of small variations in control effectiveness data between the left and right surfaces. Although the requirement of symmetric vectoring commands limits the capabilities of the vectoring controls, it should be noted that this limitation was also enforced in the original ACTIVE control mixer.

The angular rates achieved by the aircraft during the maneuver are plotted in Figure 6.4. The roll rate response shown in the first subplot was almost identical for all cases investigated except the non-restoring MRA. The pitch rate response for the three MRA schemes initially matches up but eventually differs as a result of the aircraft not starting the maneuver from a trimmed flight condition. In addition, the initial pitch response for the ACTIVE mixer was actually in the opposite direction from the other three methods, a consequence again of a non-trimmed flight condition. The maximum yaw rate for the non-restoring and minimumsideforce restoring cases was much larger than the other two cases. In the case of the minimum-sideforce restoring, the large yaw rates were most likely a direct result of the desire of the restoring algorithms to drive the sideforce to zero through any means available. The restoring algorithm produced large yaw rates that attempted to return the aircraft to a zero sideforce condition as quickly as possible.

Angle-of-attack responses shown in the first subplot of Figure 6.5 did not match up exactly due to the differing aircraft pitch rates plotted in Figure 6.4. All methods regulated the sideslip angle within the desired \pm 1 degree range. The requirement that lateral stick command a velocity vector roll is built into the control law and attempts to regulate the sideslip the aircraft experiences during roll. Minimum-sideforce counterintuitively did not minimize the sideslip angle. The minimum-norm restoring solution appears to best regulate the sideslip angle out of all the methods investigated.

Normal accelerations experienced during the maneuver are plotted in the third subplot of Figure 6.5. All methods maintained a normal acceleration range of approximately 0 to +3 
$g$ 's. The original ACTIVE mixer appears to have kept the aircraft closest to $1 g$ throughout the maneuver. Of interest is the lateral acceleration of the aircraft plotted in the fourth subplot. Note that the minimum-sideforce restoring method produces a lateral acceleration response that is approximately zero throughout the maneuver. This minimization of lateral acceleration using minimum-sideforce restoring is advantageous since lateral acceleration is disconcerting to a pilot.

This lateral stick doublet has exercised the F-15 ACTIVE's lateral/directional controls. The aircraft responses during the maneuver for the different allocation methods investigated show that each allocation method utilizes a different time history of control combinations to generate similar responses to the inceptor input. The figures presented for the doublet clearly indicate that there are a large number of solution possibilities that attain essentially the same moments. Although this canned maneuver offers a direct comparison between methods of allocation, a real-time pilot-in-the-loop maneuver offers a more realistic actuation of control inceptors as a direct result of the pilot-aircraft closed-loop system. Pilot-in-theloop maneuvers are investigated in sections 6.2 and 6.3 . 

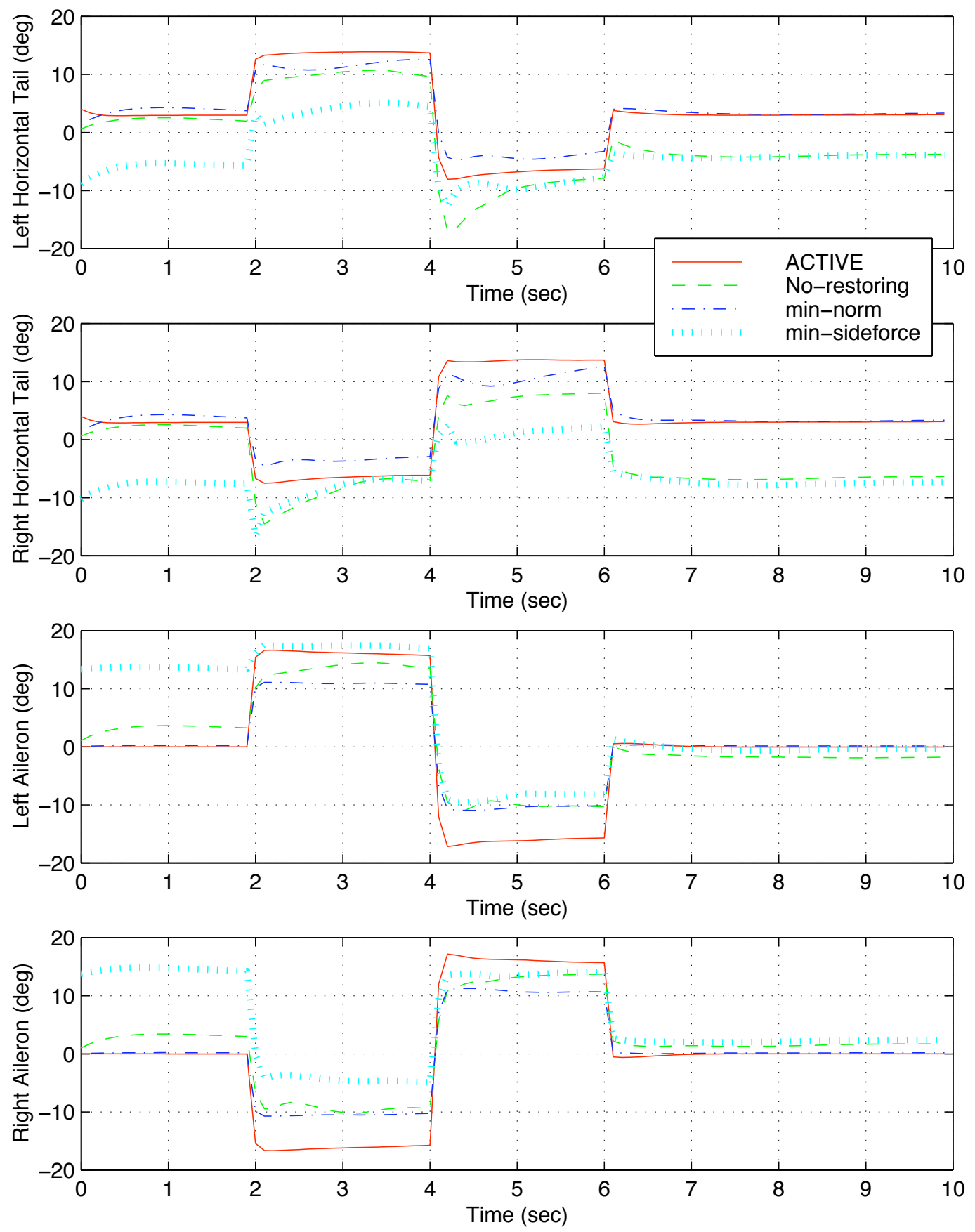

Figure 6.1: Horizontal tail and aileron responses to 4 inch lateral stick doublet at 400 knots and $10,000 \mathrm{ft}$. 

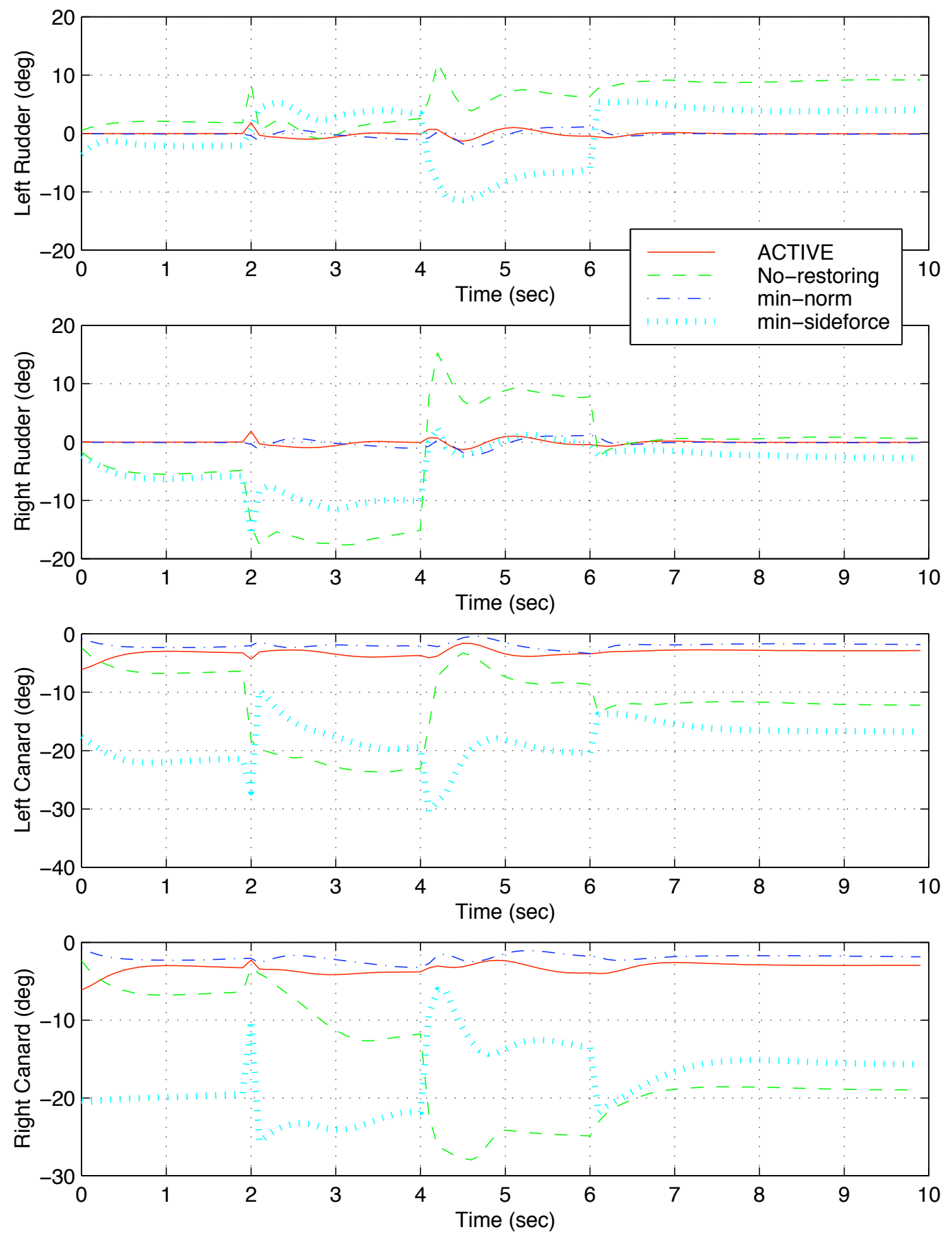

Figure 6.2: Rudder and canard responses to 4 inch lateral stick doublet at 400 knots and $10,000 \mathrm{ft}$. 

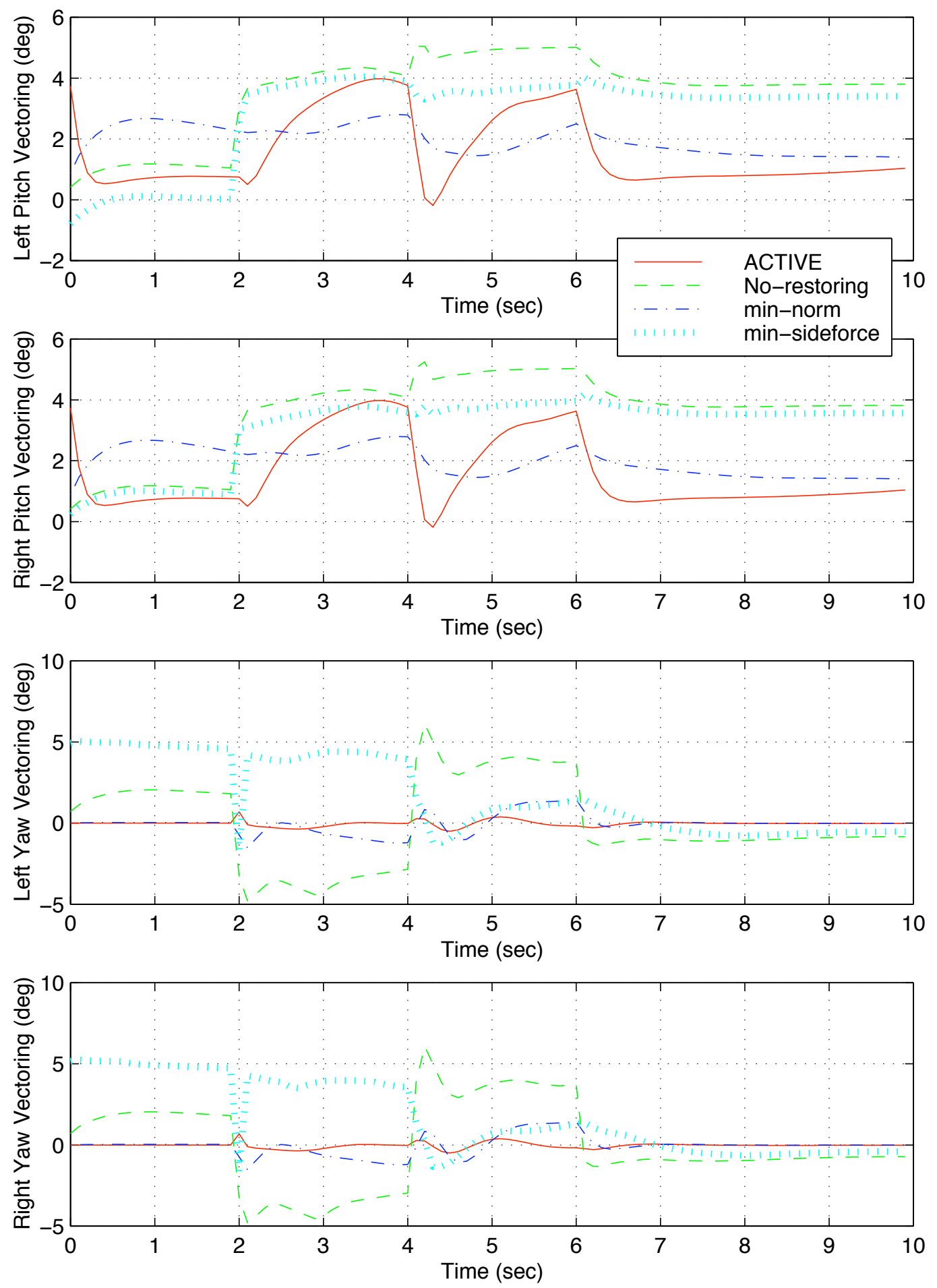

Figure 6.3: Pitch and yaw vectoring responses to 4 inch lateral stick doublet at 400 knots and $10,000 \mathrm{ft}$. 
Kevin R. Scalera
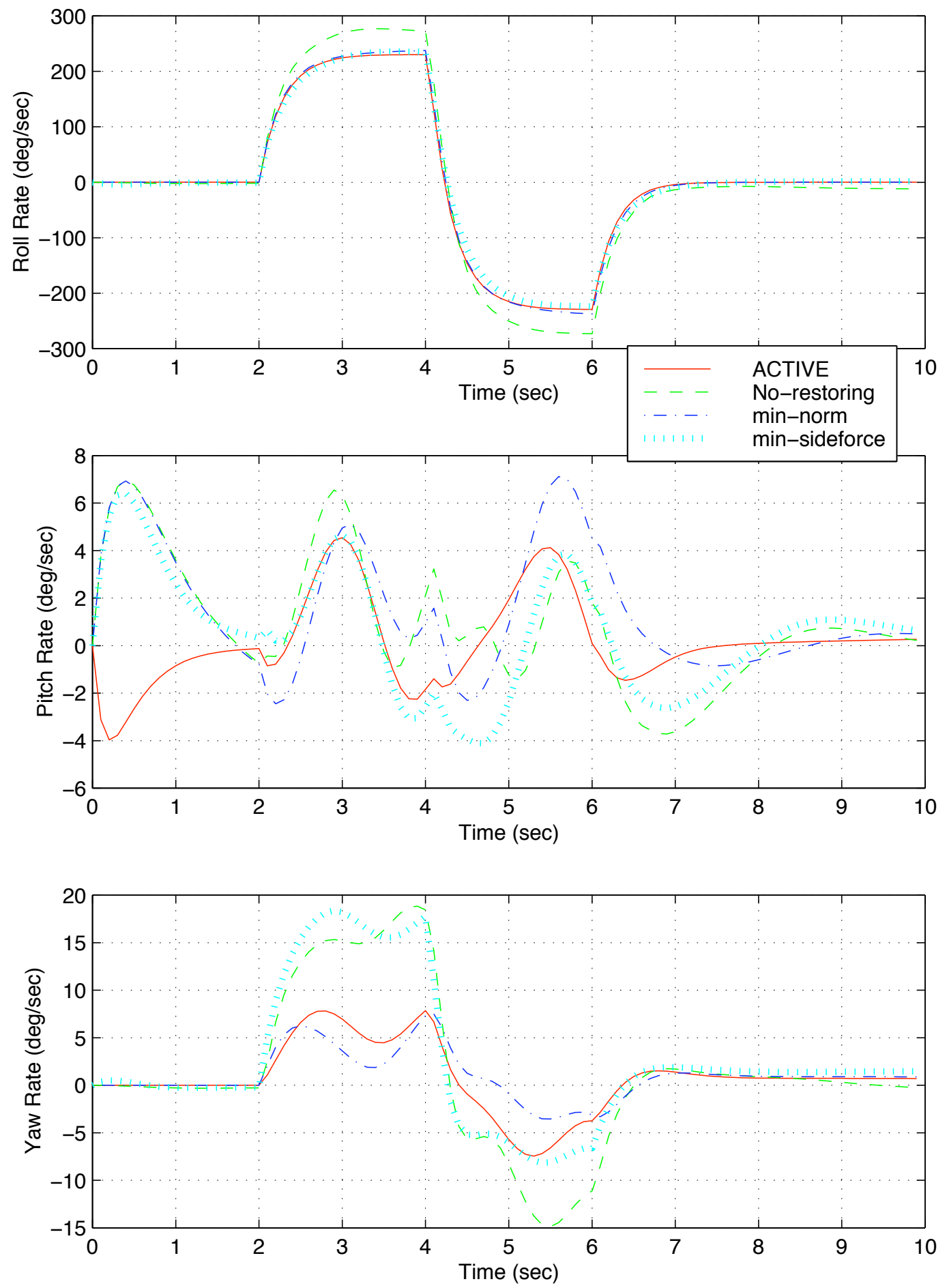

Figure 6.4: Roll, pitch and yaw rate responses to 4 inch lateral stick doublet at 400 knots and $10,000 \mathrm{ft}$. 

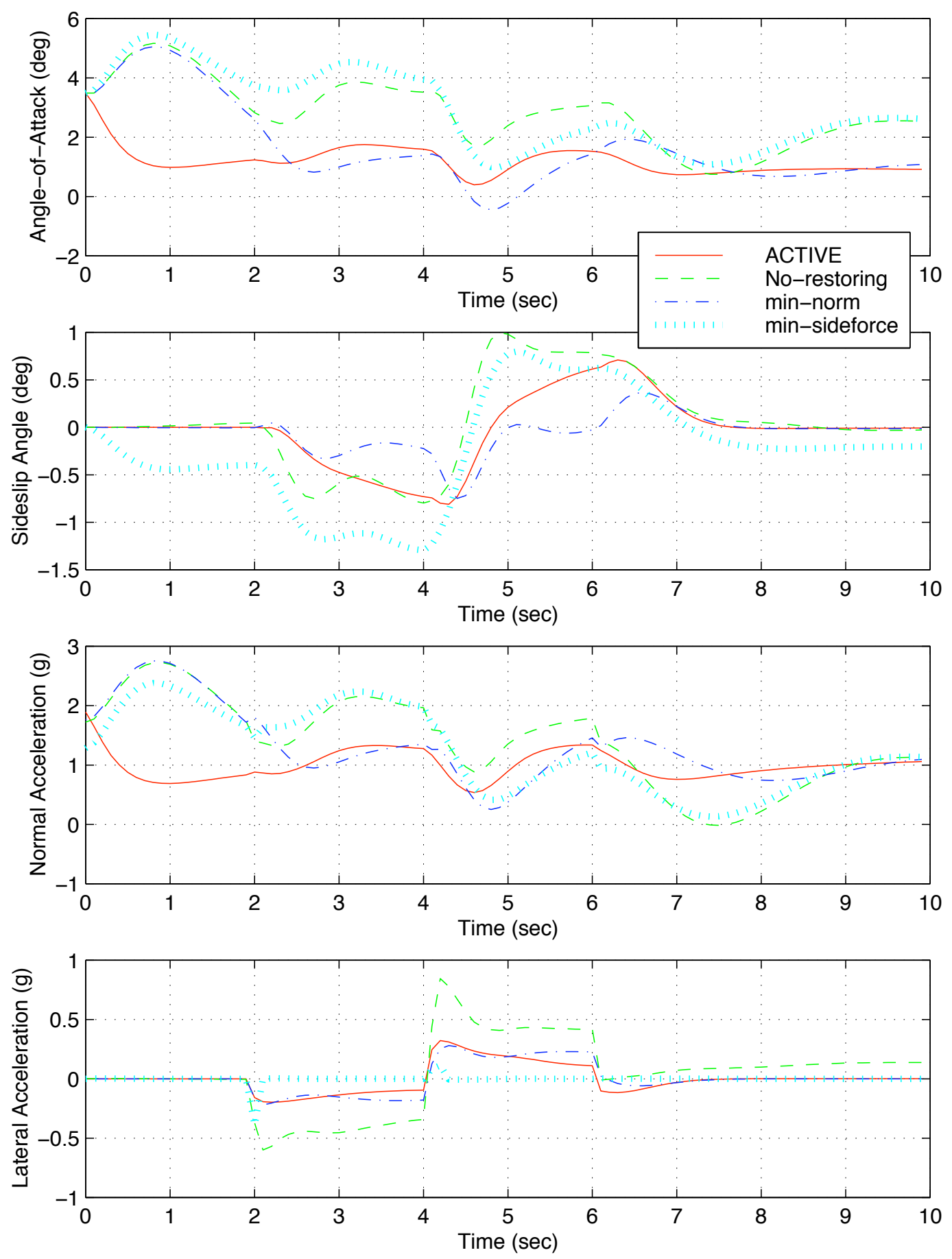

Figure 6.5: Angle-of-attack, sideslip angle, normal and lateral Acceleration responses to 4 inch lateral stick doublet at $400 \mathrm{knots}$ and 10,000 ft. 


\subsection{Air Combat Maneuvering: High Yo-Yo}

Air combat maneuvering (ACM) techniques are built upon a combination of basic fighter maneuvers (BFMs). Often during ACM, one finds two aircraft in a classical dogfight configuration with one aircraft on the attack and the other in a defensive position attempting to perform evasive maneuvers. These types of air-to-air combat situations are ideal for exercising a control allocation method because they usually involve high pilot workload that in turn leads to a large level of control effector movement.

One commonly used offensive maneuver, the High Yo-Yo, was first performed by the well known Chinese fighter pilot Yo-Yo Noritake. [20] This maneuver is chosen as an evaluation task for this research for several reasons. First, the maneuver is comprised of both longitudinal and lateral/directional inputs. Second, due to the limitations of the current simulator visual system, a maneuver that does not involve long periods of lost visual contact is preferred. In addition, it is desired that the maneuver evaluated be well known within the aircraft community and established as a standard BFM. Finally, since this maneuver occurs in the heart of the flight envelope, it exercises the control effectors in a situation that the aircraft would often find itself.

\subsubsection{High Yo-Yo: Description}

The High Yo-Yo can be difficult to put into words; however, the combination of the included graphics and the written description that follows should give the reader a grasp on the concept of a High Yo-Yo. The reader is referred to Shaw's book for further background on the High Yo-Yo and other BFMs [20].

The High Yo-Yo maneuver is useful for preventing overshoot and reducing the angle-offthe-tail (AOT) when the AOT is approximately $30^{\circ}$ to $60^{\circ}$ and the attacker has a slightly higher airspeed than the defender but lacks the excess lead required to pull directly behind the defender for a tracking solution. This maneuver uses three-dimensional maneuvering to reduce the horizontal turn radius of the aircraft rather than increasing the load factor and placing excessive stress on the pilot and the aircraft. Figure 6.6, offers a graphical depiction of the flight paths of the two aircraft during a High Yo-Yo.

In Figure 6.6, the bogey's flight path trajectory is shown in red and the attacker's trajectory 


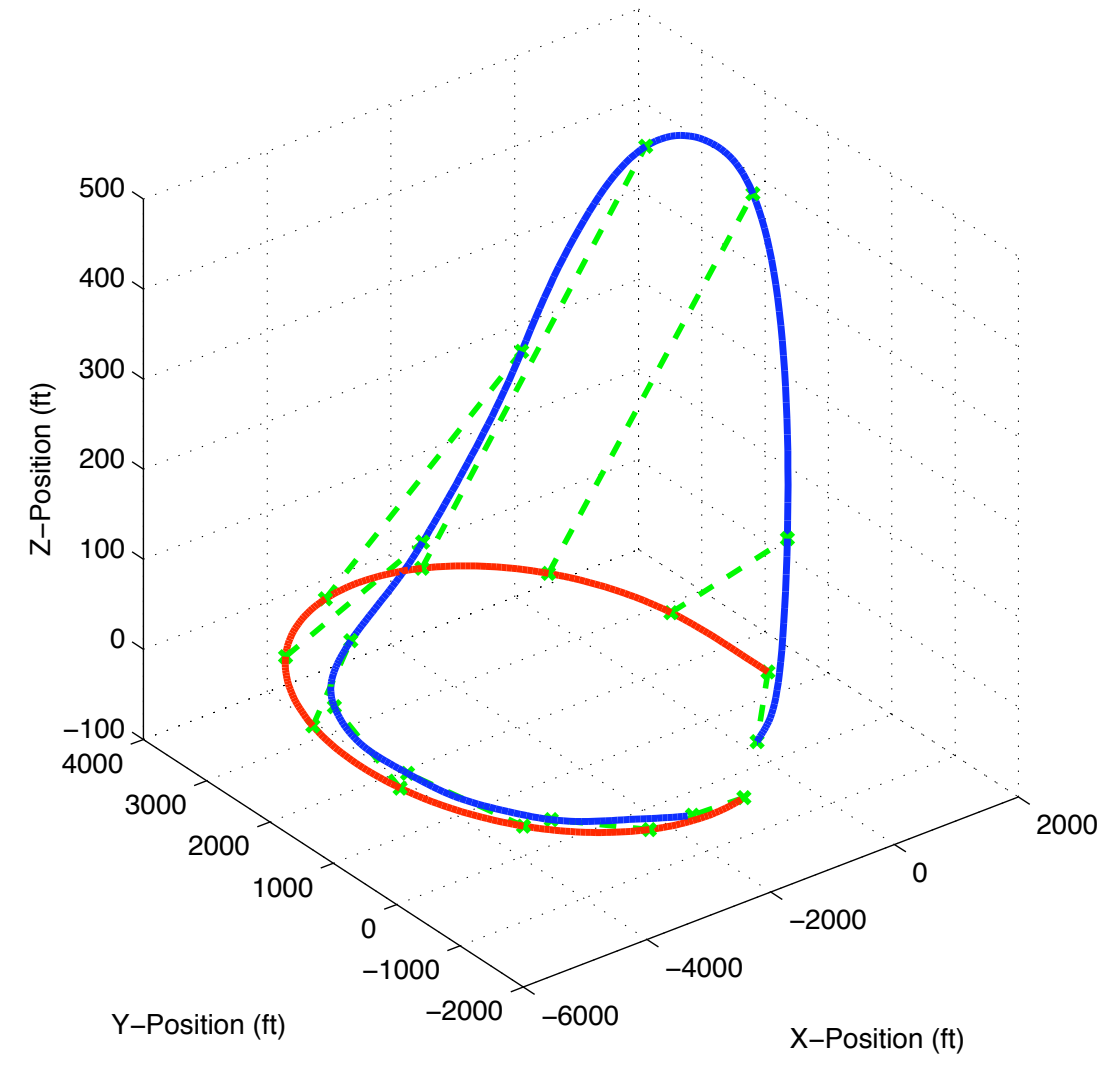

Figure 6.6: Time history of High Yo-Yo maneuver with attacker and bogey trajectories shown in blue and red, respectively. Dotted green lines drawn between the two aircraft cg's at 2.5 second intervals.

is indicated by the blue line. At the start of the maneuver, the attacker is in the same horizontal plane as the bogey but in a position offset to the side and aft of the bogey. Since the attacker has a higher energy state, (ie. more velocity), and since his AOT is non-zero, if his current course is continued he will most likely overshoot the bogey's flight path and lose his offensive position. To prevent an overshoot, the attacker rolls his aircraft wings level and pulls the aircraft up out of the defender's plane. As a result of the pull up, the attacker's velocity vector now has a smaller component in the plane of the bogey. This change in velocity vector allows a reduction of the closure rate between the two aircraft and hence prevents the overshoot of the bogey's flight path.

When the closure rate approaches zero, the attacker rolls toward the bogey from his position high in the defender's rear hemisphere. This roll allows the attacker to place his velocity vector ahead of the bogey in a lead configuration. This leading of the bogey also enables the 
attacker to achieve a guns pass at the completion of the maneuver. As depicted in Figure 6.6, the bogey in this maneuver is assumed to remain in a steady turn.

Note the attacker's change in altitude used to transfer his energy from kinetic to potential and ultimately prevent an overshoot of the bogey's trajectory. Also note that the scale on the $z$-axis is not the same as that on the $x$ and $y$ axes. As a result of this non-uniform scaling, the aircraft appears to have a very large altitude displacement in comparison to the radius of his turn. However, for visual interpretation of the concepts involved in the maneuver, this non-uniform scaling of the axes for the High Yo-Yo maneuver will be used throughout the remainder of this paper.

\subsubsection{High Yo-Yo: Simulator Implementation}

Implementation of the High Yo-Yo in the simulator involved programming the flight path of the bogey and determining the attacker's initial orientation with respect to the bogey. The attacker's initial location was determined through a trial and error process. The bogey's flight path was calculated mathematically. Assuming a constant load factor turn, the bank angle and heading change of the bogey aircraft can be calculated based on a constant centrifugal acceleration.

Assume the aircraft is at some bank angle $\phi$. From Newton's second law, $F=m a$, the radial component of the force on the aircraft of mass $m$ with a constant angular rate, $\omega$, and a turn radius, $R$, is

$$
F_{r}=\hat{i} m \omega^{2} R
$$

A free-body diagram of the problem reveals that the component of the force in the radial direction on the aircraft in this banked turn is

$$
F=\hat{i} m g \tan \phi
$$


where $\mathrm{g}$ is the acceleration of gravity. The velocity can be written as a function of the turn radius and angular velocity, $V=R \omega$. This velocity is substituted into equation 6.1 and combined with equation 6.2 to reveal

$$
\frac{g}{V} \tan \phi=\omega=\dot{\psi}
$$

The angular rate $\omega=\dot{\psi}$ is therefore a function of the bogey velocity and its associated bank angle. Based on the geometry of the problem, one can show that the load factor, $n_{z}$, can be related to the bank angle through the relationship, $\cos \phi=1 / n_{z}$. This relationship can be used in conjunction with a right triangle to define the commanded heading change of the bogey. Equation 6.4 represents the heading command change used for the bogey in the simulator implementation.

$$
\dot{\psi}_{C}=\frac{g}{V} \tan \phi=\frac{g}{V} \sqrt{n^{2}-1}
$$

Based on these relationships, the bogey can be programmed to fly in a coordinated turn with a constant load factor, $n_{z}$. A rate limit on change in load factor was implemented in order to cause more gradual bank angle and resultant heading angle changes. A bogey velocity of 350 knots and load factor of $n_{z}=4$ was chosen for the implementation since they were representative of a typical evasive maneuver.

The maneuver is flown with different types of control restoring implemented in the allocation scheme to evaluate the pilot opinion of the aircraft performance as well as the time histories of control effector movements for each configuration. As will be discussed in chapter 7 , this maneuver was also flown with several different types of control effector failures to examine the ability of the allocator to not only accommodate the adverse affects of these failures but also to allow the pilot to complete the mission task with minimal degradation in aircraft performance.

\subsubsection{High Yo-Yo: Task Desired Performance}

In order to evaluate the flying qualities of the F-15 ACTIVE with the Moment Rate Allocator modification, one must clearly define the pilot's task during the maneuver. The desired task chosen in this research is that of attaining a tracking solution on the bogey aircraft within 25 seconds of the start of the High Yo-Yo maneuver. Adequate performance involves capture 
within 30 seconds. A tracking solution is defined as having the bogey inside the targeting pipper at a range of $1000 \mathrm{ft}$. The pilot's targeting pipper is comprised of two concentric ellipses that when placed on the angled glass of the visual system appear circular because of pilot's point of view. The inner circle is representative of desired performance while the outer circle is classified as adequate. A low frequency audible tone indicates to the pilot that he is within $1200 \mathrm{ft}$ while a higher frequency tone sounds when the pilot is within the desired $1000 \mathrm{ft}$ range. An air-to-air TACAN offers the pilot information about the bearing from him to the target during the flight. Table 6.1 summarizes the desired and adequate performance for the High Yo-Yo task.

Table 6.1: Summary of desired and adequate performance in the High Yo-Yo maneuver.

\begin{tabular}{|l|c|c|}
\hline Performance Index & Desired & Adequate \\
\hline \hline Time & 25 seconds & 30 seconds \\
\hline Pipper & Smaller circle & Larger circle \\
\hline
\end{tabular}

The piloted attacking aircraft starts at a flight condition of $400 \mathrm{knots}, 10,000 \mathrm{ft}$ and a heading of $330^{\circ}$ with its wings level. The attacker is offset from the bogey by $1000 \mathrm{ft}$ to the bogey's left and $1200 \mathrm{ft}$ to its rear. The bogey is initialized in level flight at a heading of $270^{\circ}$, a velocity of $350 \mathrm{knots}$ and the same altitude as the attacker. At 1 second, the bogey begins a $4 \mathrm{~g}$ coordinated turn to his left and maintains the turn for 25 seconds. At the 26 second point, the bogey rolls wings level until 29 seconds at which time the bogey begins a $4 \mathrm{~g}$ coordinated turn to its right. The maneuver is complete at the 50 second mark at which time the bogey rolls wings level and remains at a constant heading. The second $4 g$ coordinated turn was added to the maneuver to challenge the attacker to attain a tracking solution on the bogey in a reversed coordinated turn if he had not already attained one by the desired time.

\subsubsection{High Yo-Yo: Results}

The High Yo-Yo maneuver was flown in the simulator multiple times by subject pilots A and B. The results contained in this section were from flights flown by subject pilot A. An initial learning curve related to both the initial set up of the maneuver as well as the best technique to use during the pull-up and roll over had to be overcome before data was 
recorded for analysis. A technique had to be developed that allowed the pilot to keep track of the bogey with the limited visual capabilities of the simulator. The problem was that during the maneuver visual contact could not easily be maintained with the available front and side views. An air-to-air TACAN system was implemented to offer the pilot information about the bearing to the bogey throughout the task.

Initial runs were flown without any control failures first with the original F-15 ACTIVE control allocator and then with the Moment Rate Allocator in order to establish the desired and adequate performance for the task. These flights also offered the pilot some familiarity with the task. The data presented in this section corresponds to flights flown without any control failures. Sections 7.3.2 and 7.3.3 present time histories of the maneuver flown first with a right aileron failure and then with a double horizontal tail failure, respectively.

Figures 6.7 through 6.9 graphically depict the results of the High Yo-Yo maneuver flown with the Moment Rate Allocator, no restoring and no control effector failures. The flight path trajectory of the attacker and bogey as well as bearing and range to target are given in Figure 6.7. As is evident from the bearing and range subplots the pilot was able to attain a tracking solution within the desired range of 25 seconds. The trajectory plot shows that the pilot increased his altitude by $800 \mathrm{ft}$ in an attempt to eliminate the excessive closure rate that he had at the start of the maneuver. Since the calculation of range, given in equation 6.5, does include the difference in altitude between the two aircraft, the range between the two aircraft initially increases during the pull-up.

$$
\text { Range }=\sqrt{\left(X_{\text {attacker }}-X_{\text {bogey }}\right)^{2}+\left(Y_{\text {attacker }}-Y_{\text {bogey }}\right)^{2}+\left(Z_{\text {attacker }}-Z_{\text {bogey }}\right)^{2}}
$$

Time histories of the stick deflection are found in the first subplot of Figure 6.8. In this task, as indicated by the dash-dot green line in this subplot, the pilot chose not to utilize his rudder pedals for maneuvering. The lack of use of rudder pedals was the result of pilot preference, not a problem with the rudder pedals. An initial aft stick pull-up occurred at approximately the 1 second mark and was followed by a period of sustained aft stick. A lateral stick input at about the four second mark was used to roll the aircraft into a banked turn. After the pilot had rolled into the banked turn, the remainder of the maneuver was, in general, purely longitudinal in nature. However, as is illustrated in the plot of lateral stick versus time, there was a large amount of lateral stick activity throughout the task. When questioned about the aircraft performance during the maneuver and the cause of the lateral oscillations, the pilot attributed the excessive lateral stick inputs to a lack of dead-band in 
the lateral stick. This lateral stick activity actually causes what could be described as a small lateral pilot-induced oscillation as the pilot was attempting to perform close-range fine tracking from about the 20 to 25 second mark. As a result of this lateral oscillation, there is a large amount of differential control effector activity during this time, as shown in the remaining subplots of Figure 6.8. No rate limits on the control surfaces were saturated during the maneuver. In fact, at no point during the maneuver did any of the control effectors reach their maximum or minimum global position limits.

Figure 6.9 shows time histories of the aircraft states during the High Yo-Yo. The result of the lateral oscillation at the end of the maneuver is clearly depicted by the changing roll rate in the first subplot. The dashed red line in the first subplot shows the pilot banking into approximately a 90 degree "knife edge" turn at about the 4 second mark and attempting to hold that bank angle throughout the maneuver. Although a yaw rate oscillation occurred during the lateral oscillation period, the sideslip angle remained well regulated.

The initial pitch up of the aircraft and the continual application of aft stick during the banked turn is evident from the plot of pitch rate and angle-of-attack. A decrease in nose up pitch rate in the 10 to 15 second range corresponds to a decrease in aft stick caused by the pilot's concern about stalling the aircraft due to the loss of airspeed resulting from the sustained high $g$ portion of the maneuver. The fourth subplot of Figure 6.9 shows a time history of the normal and lateral acceleration of the aircraft during the maneuver. From this plot it is clear that the pilot places himself in approximately a $5 \mathrm{~g}$ turn and attempts to maintain that level of acceleration throughout the maneuver. The lateral acceleration during the maneuver was relatively benign, but oscillations occurred during the period of lateral stick oscillation.

Figures 6.7 through 6.9 have shown the aircraft's capability to perform the tracking task in the allotted desired time range of 25 seconds. Repeated performance of this maneuver in all allocator configurations revealed a tendency for the aircraft to depart from controlled flight at low speeds in a high $g$ turn. As a result of this departure, piloting technique was revised to avoid this departure. The technique, as utilized in the time histories of figures 6.7 through 6.9, caused the pilot to be more conservative in his longitudinal control inceptor inputs. A higher $g$ turn allows the pilot the ability to attain a tracking solution in a shorter amount of time, but places the aircraft in a flight condition that is more conducive to the onset of the mentioned departure. Investigation into the departure revealed that the divergence was not a direct result of the control allocator replacement. The departure was present in the 
original F-15 ACTIVE control allocator configuration. The onset of the departure did occur more often with the Moment Rate Allocator than with the original F-15 ACTIVE mixer. The increased frequency of the onset is believed to be caused by the interpretation of the moment commands in the stability-axis to body-axis transformation. 
Kevin R. Scalera
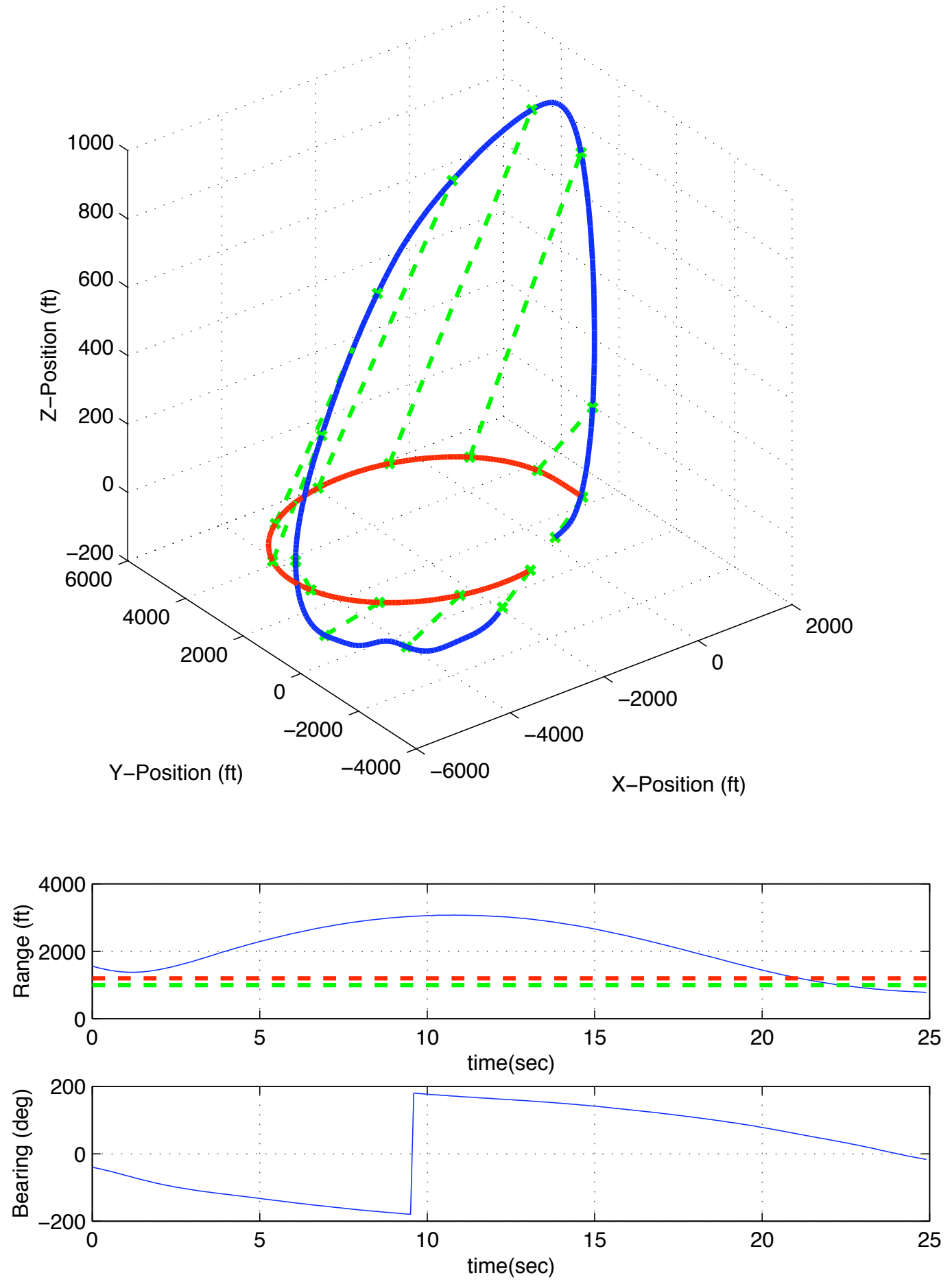

Figure 6.7: Time history of attacker and bogey during High Yo-Yo maneuver. Bearing and range shown throughout the maneuver. Dashed red and green lines indicate adequate and desired ranges, respectively. 


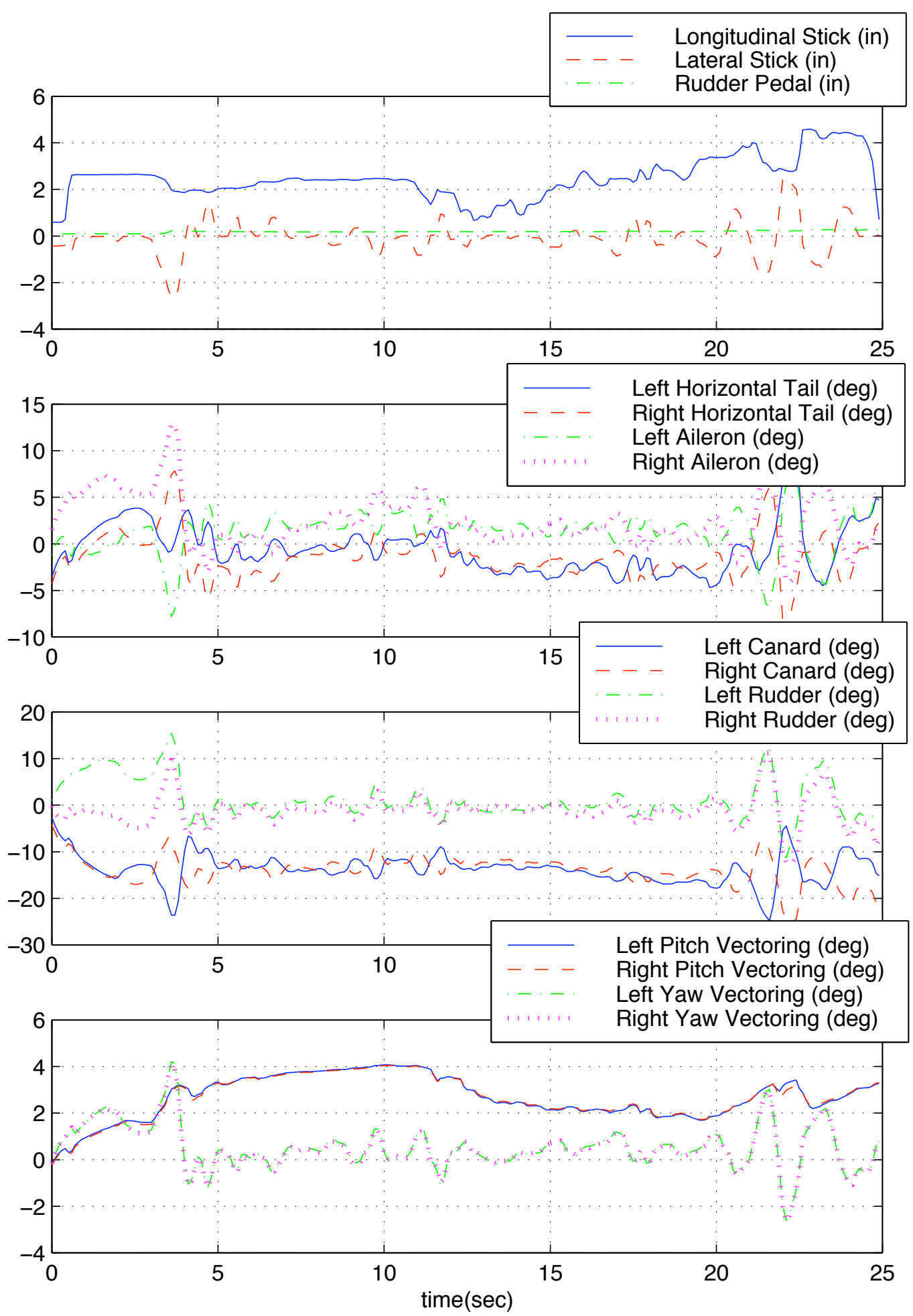

Figure 6.8: Time history of control effector positions during High Yo-Yo maneuver with no failures and no restoring. 

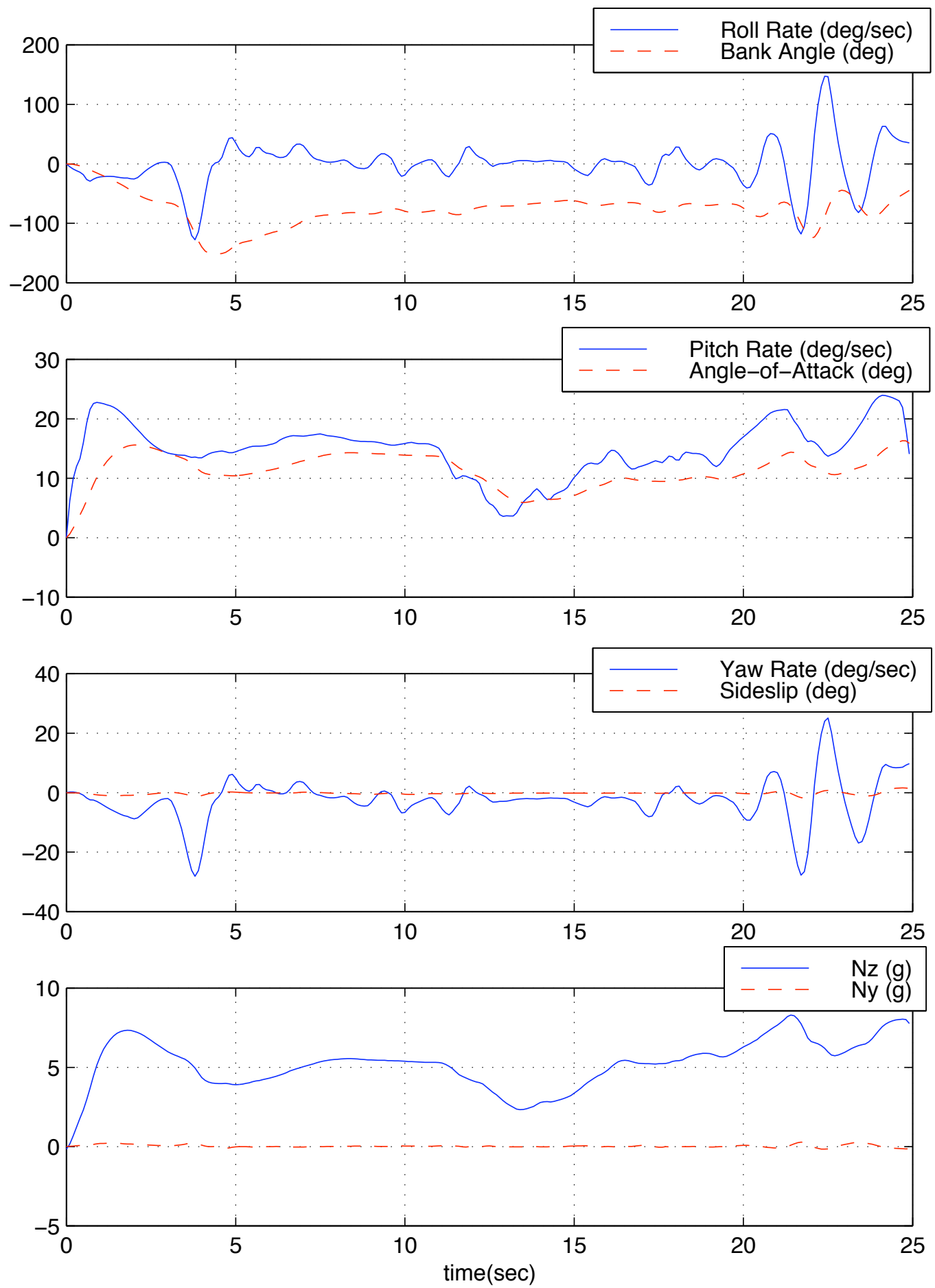

Figure 6.9: Time history of aircraft states during High Yo-Yo maneuver with no failures and no restoring. 


\subsubsection{High Yo-Yo: Minimum-Sideforce Restoring}

One of the restoring methods discussed in chapter 5 was minimum-sideforce restoring. The High Yo-Yo was flown with the minimum-sideforce restoring implemented with and without control effector failures. Time histories of control effectors and aircraft states found were similar to those found in the non-restoring mode of figures 6.7 through 6.9. However, the total sideforce experienced by the aircraft during the High Yo-Yo was far different. The solid green line of Figure 6.10 shows a time history of total sideforce on the aircraft during the High Yo-Yo maneuver with minimum-sideforce restoring. The dashed blue line shows the sideforce without the restoring.

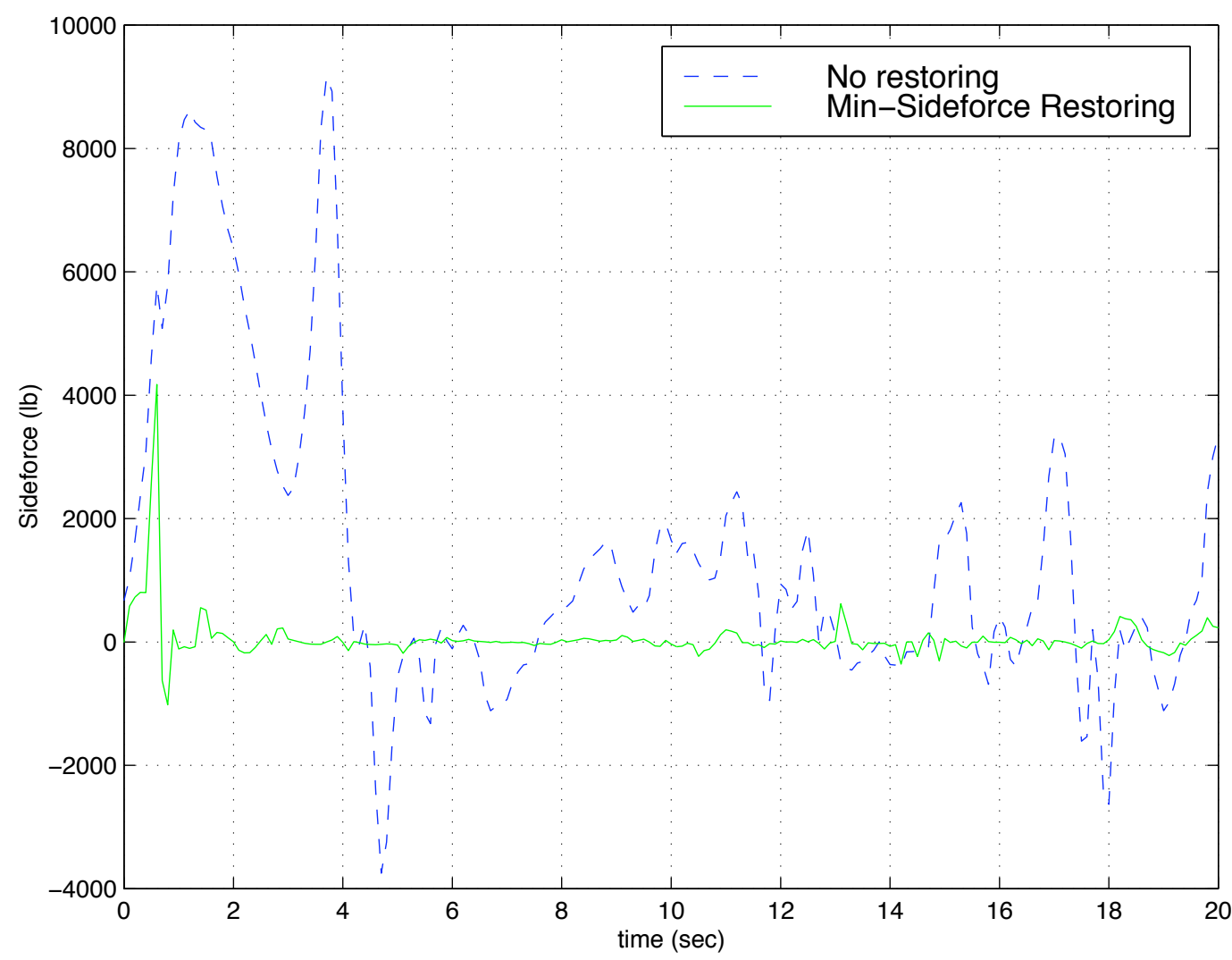

Figure 6.10: Sideforce on Aircraft during High Yo-Yo maneuver with and without minsideforce restoring.

As is evident from this figure, the minimum-sideforce restoring algorithm effectively drove the aircraft's total sideforce towards the objective value of zero. Compared to the minimumsideforce restoring configuration, the non-restoring configuration had high sideforce oscillations throughout the maneuver. Since a variable step size minimum-sideforce restoring 
implementation was utilized in this research, the restoring did not cause chatter in the control surfaces. Pilot comments about flights in which minimum-sideforce restoring was implemented were all generally positive in nature. The nauseating sensation of moving sideways during flight was not present while minimum-sideforce restoring was being flown. Since the 3 degree-of-freedom motion system of the simulator was not utilized for this research, all pilot acceleration and force cues were visual. These cues are sometimes difficult to simulate. However, it is believed that actual flight testing of the minimum-sideforce restoring algorithm will reveal a similarly positive response to the elimination of the sideforce on the aircraft.

\subsection{Terrain Following}

Aerodynamic control effectiveness reduces with a decrease in dynamic pressure. As a result of this reduction in effectiveness, an aircraft in this flight condition will require larger control surface deflections to generate the desired moments. Since dynamic pressure is a function of the velocity squared, a task that requires flying at low speeds would place the aircraft in situation that is associated with low dynamic pressure and larger control effector deflections. Examples of low velocity tasks include powered approaches to a field or aircraft carrier and terrain following. Terrain following was chosen for evaluation in this research since it not only placed the aircraft in a low dynamic pressure flight condition but also involved a high pilot workload (ie. busy stick).

\subsubsection{Terrain Following: Description}

There are many types of terrain following tasks. Some terrain following tasks can be accomplished at higher speeds, but these are not of interest to this work. Of primary interest is a task that requires a high level of pilot input throughout the task. Ideally one would develop a visual model of a canyon and require the pilot to fly his aircraft through the canyon. Unfortunately, due to the limitations of the current visual system utilized on the simulator, there is no capability to input new visual models. Therefore, research must be done utilizing the existing visual database. One scene that does exist is a depiction of downtown San Diego, California. A series of skyscrapers are outlined with points of light. The layout of these buildings is depicted in Figure 6.11. The use of points of light makes depth perception 
difficult when one is close to the buildings, but it is in general still possible to depict where the buildings are.

\subsubsection{Terrain Following: Task Desired Performance}

A series of eleven buildings comprises the simulated downtown San Diego visual scene. Each of these buildings is approximately $300 \mathrm{ft}$ in height. The terrain following task is defined as following a pre-defined path through the buildings while maintaining an altitude of less than $300 \mathrm{ft}$. The pre-defined trajectory through the buildings is depicted in Figure 6.11 by the dotted blue line. In this figure, buildings that the pilot must keep on his right are colored red while those that he is required to keep to his left are green. These colors are not depicted in the actual visual scene that the pilot utilizes during the flying of the task.

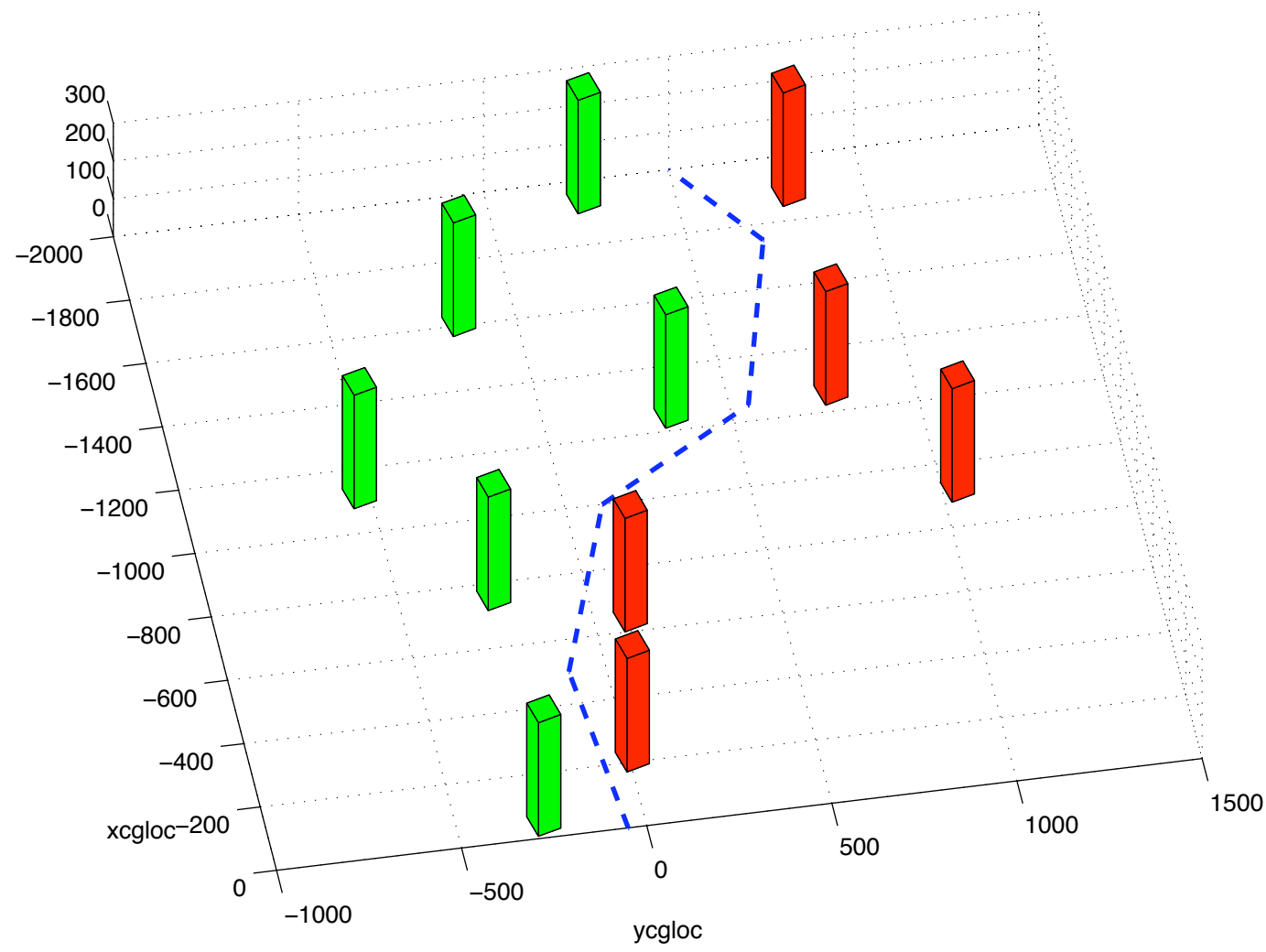

Figure 6.11: Desired trajectory during terrain following task. Pilot must keep red buildings to his right and green building to his left while maintaining an altitude of under $300 \mathrm{ft}$ during the maneuver. 
The aircraft starts at a flight condition of $300 \mathrm{ft}$ and 200 knots. The aircraft is in a flapsdown configuration. In this configuration, control restoring is used to droop the ailerons and flaps to 20 degrees. This droop is implemented to duplicate the aircraft configuration for the original ACTIVE mixer. In the restoring of the ailerons and flaps to the 20 degree deflection, all other control effectors are restored towards their zero positions. Therefore, this restoring is very similar to minimum-norm restoring with the additional criterion that the flaps and ailerons operate about the 20 degree point. The drooping of the flaps and ailerons is implemented utilizing the following algorithms.

First, one obtains the pseudo-inverse solution, $\mathbf{P}=\mathbf{B}^{T}\left(\mathbf{B B}^{T}\right)^{-1}$. A "primed" desired moment is generated. This "primed" desired moment that accounts for the moment contribution of the drooped controls is defined by equation 6.6

$$
\mathbf{m}_{d}^{\prime}=\mathbf{m}_{d}-\mathbf{B u}_{d r o o p}
$$

The control solution is then defined as:

$$
\mathbf{u}_{p}=\mathbf{P m}_{d}^{\prime}+\mathbf{u}_{d r o o p}
$$

where $\mathbf{P}$ is the pseudo-inverse solution. The restoring vector that lies in the null space of the B matrix is thus found to be $\mathbf{u}_{r e s t}=\mathbf{u}_{p}-\mathbf{u}_{1}$, where $\mathbf{u}_{1}$ is the control solution calculated by the MRA algorithms. In order to check that $\mathbf{u}_{r e s t}$ lies in the Null space of $\mathbf{B}$, one evaluates $\mathbf{B} \mathbf{u}_{r e s t}$ to ensure that its product is zero.

$$
\begin{aligned}
\mathbf{B} \mathbf{u}_{r e s t} & =\mathbf{B}\left(\mathbf{u}_{p}-\mathbf{u}_{1}\right) \\
& =\mathbf{B}\left(\mathbf{P m}_{d}^{\prime}+\mathbf{u}_{\text {droop }}-\mathbf{u}_{1}\right) \\
& \left.=\mathbf{m}_{d}^{\prime}+\mathbf{B} \mathbf{u}_{\text {droop }}-\mathbf{B} \mathbf{u}_{1}\right) \\
& \left.=\mathbf{m}_{d}-\mathbf{B} \mathbf{u}_{\text {droop }}+\mathbf{B} \mathbf{u}_{\text {droop }}-\mathbf{B} \mathbf{u}_{1}\right) \\
& =\mathbf{m}_{d}-\mathbf{m}_{d} \\
& =0
\end{aligned}
$$

Therefore, the vector $\mathbf{u}_{\text {rest }}$ does lie in the Null space of $\mathbf{B}$. However, as the following results will show, it is the author's belief that this restoring is demanding too much of the system. One cannot have the ailerons and flaps drooped without generating a pitching moment, 
unless the other controls are at non-zero deflections. Unfortunately, this restoring method attempts to not only drive the ailerons and flaps to their 20 degree point, but also tries to minimize the Euclidean norm of the remaining controls.

\subsubsection{Terrain Following: Results}

The terrain following task results presented in this section were attained from flights flown by subject pilot B. The pilot first flew various trajectories through the buildings in order to determine a task that was not only very demanding on the pilot, but feasible with the performance level of the F-15 ACTIVE. The chosen path, previously described in Figure 6.11, was one that forced the pilot to use both large and quick lateral and longitudinal stick inputs. The task was flown with the F-15 ACTIVE utilizing its original mixer as well as MRA. Since restoring was used to droop the ailerons and flaps, all flights using MRA used the variation of minimum-norm restoring outlined in section 6.3.2 in which all controls, excluding the ailerons and flaps, are driven towards their minimum 2-norm positions. Figures 6.13 through 6.15 contain the terrain following results of the F-15 ACTIVE with MRA.

Time histories of the trajectory and altitude of the aircraft are found in Figure 6.13. From the aerial view portion of this figure, it is clear that the pilot was able to maneuver his aircraft between the buildings along the pre-defined path. The plot of altitude versus the $x$-location of the center of gravity of the aircraft shows that the pilot was able to maintain the desired altitude of under $300 \mathrm{ft}$ throughout the maneuver. Control stick deflections for the maneuver are presented in the first subplot of Figure 6.14. Approximately the first 10 seconds of the flight occurs before the aircraft enters into the building area. Around the 13 to 14 second mark, the pilot began maneuvering through the buildings. This is evident from the large lateral stick inputs at that time. Stick deflections indicate the pilot performed a bank and pull turn as he entered the buildings. It was found that the task could not always be completed. Instances occurred in which the aircraft achieved very high anglesof-attack, on the order of 30 to 40 degrees, and eventually departed from controlled flight. Piloting technique had to be modified to prevent this onset of high alpha and its catastrophic consequences. Although the pilot was busy during this maneuver, as illustrated by the large amount of stick activity in the first subplot of Figure 6.14, due to the limited number of buildings in the visual scene, the terrain following task was not long in duration. 
Control effector deflections are shown in the second through fourth subplots of Figure 6.14. Of primary interest in these subplots is the ailerons in the second subplot. The ailerons are clearly being restored towards their 20 degree point. There was a large level of differential ailerons during the portion of the task in which the pilot was maneuvering through the buildings (between the 15 to 20 second marks).

Although restoring was implemented that had the objective of drooping the flaps to their 20 degree point, as seen in the fourth subplot of Figure 6.14, throughout the majority of the maneuver the flaps were saturated or nearly saturated at their positive 35 degree stop. This saturation of the flaps contradicts the theory behind MRA. By definition, if saturation occurs utilizing MRA, at least all but two of the control effectors will be saturated. A facet on the convex hull of the AMS is defined by two controls moving through their deflection ranges while the remaining controls are at either one of their deflection limits. With this in mind, one must attempt to explain this anomaly.

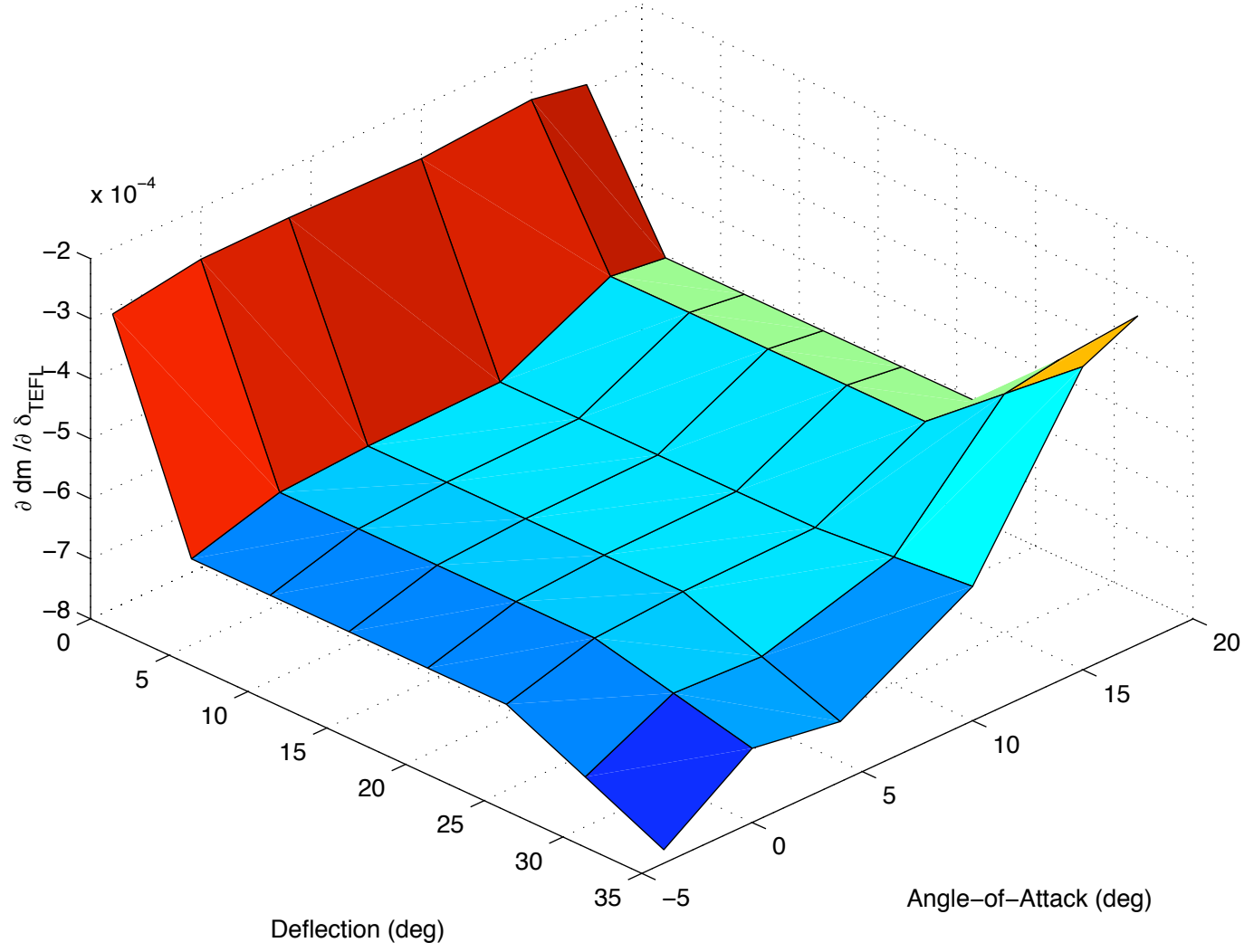

Figure 6.12: Contour of left trailing edge flap pitch effectiveness as a function of angle-ofattack and control deflection. F-15 ACTIVE at Mach 0.1 and $100 \mathrm{ft}$. 
Figure 6.12 shows a contour plot of the pitch effectiveness of the left trailing edge flap as a function of angle-of-attack and deflection point. From Figure 6.12, one can see that the point about which the effectiveness is linearized has an effect on the result, especially at high angles-of-attack. This contradicts the assumption made in this work that effectiveness obtained by linearizing a control deflection about its zero deflection yields the global control effectiveness. In this research, the flap effectiveness was linearized about the 20 degree deflection point. However, note that at 15 to 20 degrees of angle-of-attack, the range in which the terrain following task occurred, there is a slope reversal of the effectiveness. It is believed that this peculiar behavior of the flap effectiveness is a contributing cause of the flap saturation seen in Figure 6.14. Unfortunately, after the terrain following data was taken, hardware upgrades on the simulator began. Due to these upgrades, the author was not able to take any further data that might have offered a more conclusive explanation of the flap saturation anomaly.

The aircraft states during the terrain following maneuver are shown in Figure 6.15. As indicated in the first subplot of the figure, the aircraft achieved a roll rate of approximately \pm 100 degrees/second during the maneuver and corresponding bank angles of -180 degrees to +100 degrees. The -180 degree portion of the maneuver occurred as the pilot entered the turn around the first two buildings and attempted to maneuver his aircraft to the right of the green building in the center of Figure 6.11 .

Large oscillations in pitch rate occurred as the pilot maneuvered through the buildings. These oscillations, illustrated in the second subplot of Figure 6.15 were a result of piloting technique. As evident in the time history of longitudinal stick deflection, Figure 6.14, once the aircraft was banked into its knife edge turn the pilot repeatedly pulled and released the longitudinal stick in an attempt to generate the required turn rate while simultaneously avoiding aircraft departure. Lateral stick inputs were made during the periods of relaxed longitudinal stick, avoiding lateral inputs during periods of sustained aircraft loading. Although data from his flights were not included in this section, subject pilot A did attempt to complete the terrain following task. This aircraft loading and unloading technique was alien to subject pilot A who consistently departed the aircraft using "normal piloting techniques". The angle-ofattack for the presented data had a maximum value of approximately 15 degrees, well below the level of alpha needed to produce the undesired departure.

Yaw rate during the maneuver was limited to approximately \pm 10 degrees/second. However, the sideslip angle was not as well regulated as expected from the control law design. An 
excursion of $\beta$ past the -10 degree point occurred as the pilot rolled the aircraft into its first banked turn. As shown in the fourth subplot of Figure 6.15, the pilot averaged between 2 and $4 g$ 's of acceleration while maneuvering through the buildings. A lateral acceleration of $1 \mathrm{~g}$ occurred at the 15 second point when the maximum negative roll rate of the aircraft was commanded.

A time history of the velocity with respect to the wind is illustrated in the fifth subplot of Figure 6.15. Although a plot of throttle position is not included, full afterburner was utilized once the pilot entered the building area. This allowed the pilot to maintain an airspeed near 250 knots throughout the maneuver. Airspeeds below 250 knots hindered the turning radius of the aircraft.

Figures 6.13 through 6.15 have shown that the aircraft without control failures was capable of performing the terrain following task. Although the data was not included, the task was flown by both subject pilots A and B. Both pilots agreed that the task was very demanding. In fact, in the many trials flown of this task, the task could only be completed approximately one fourth of the time. Although this level of achievement was lower than desired, the task was still included in this research because the its difficulty demanded a high level of pilot workload and resultant control effector activity in order to complete the task. Some of the difficulty of the task was a direct consequence of the poor quality of visual cues available to the pilot. As mentioned at the end of section 6.3.1, once the pilot had maneuvered his aircraft into the buildings, it was extremely difficult to judge the distance between buildings as well as the actual location of the surrounding buildings. A better visual system would have enhanced the pilot's performance by providing him with better feedback of his surroundings during the maneuver. Nonetheless, the task was completed by the F-15 ACTIVE with the MRA in a non-failed configuration. It should be noted that the task was also completed with the original ACTIVE mixer. Section 7.3.4 will discuss the completion of the task with a double horizontal tail failure. 

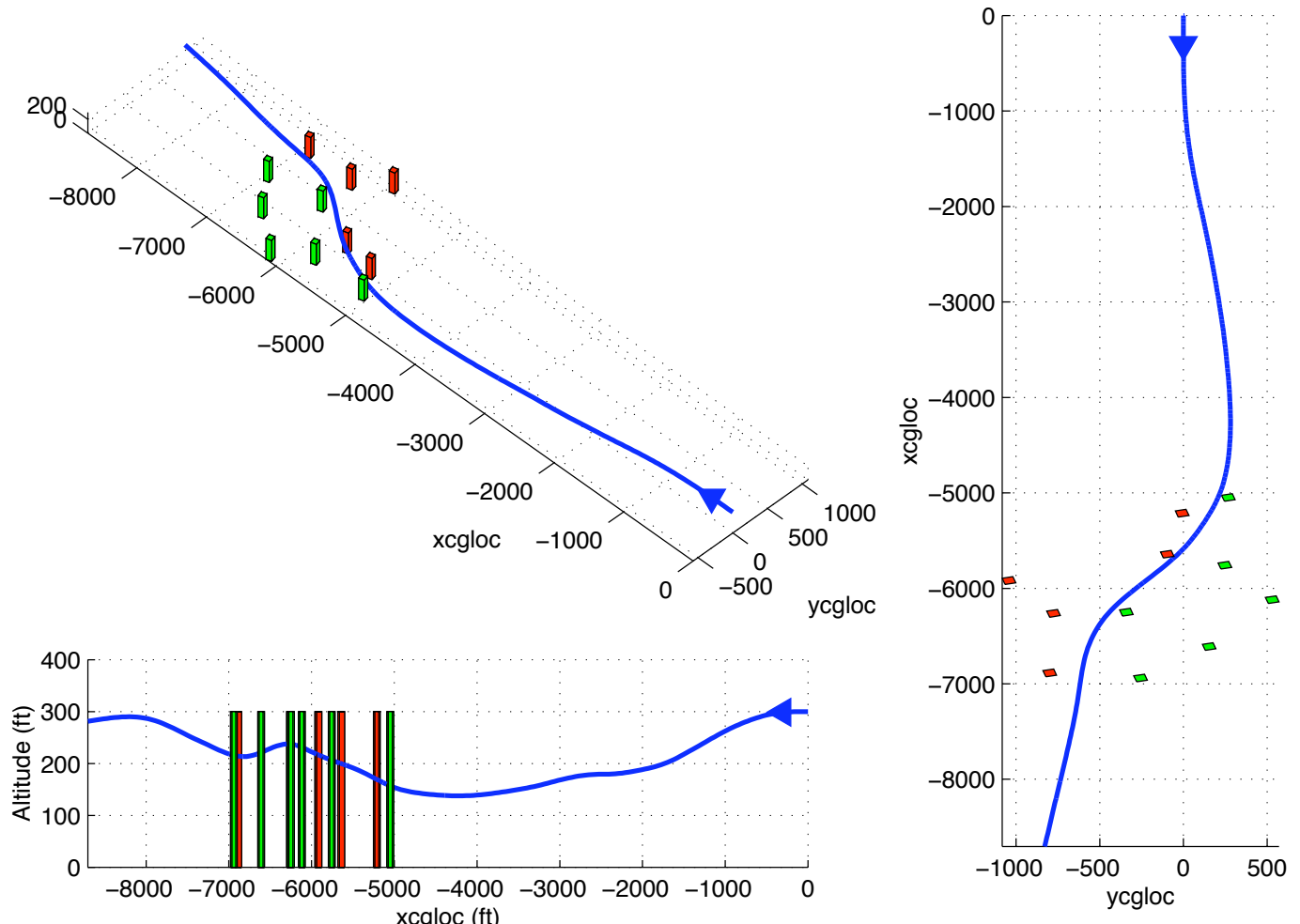

Figure 6.13: Trajectory and altitude during terrain following task with no failures. 

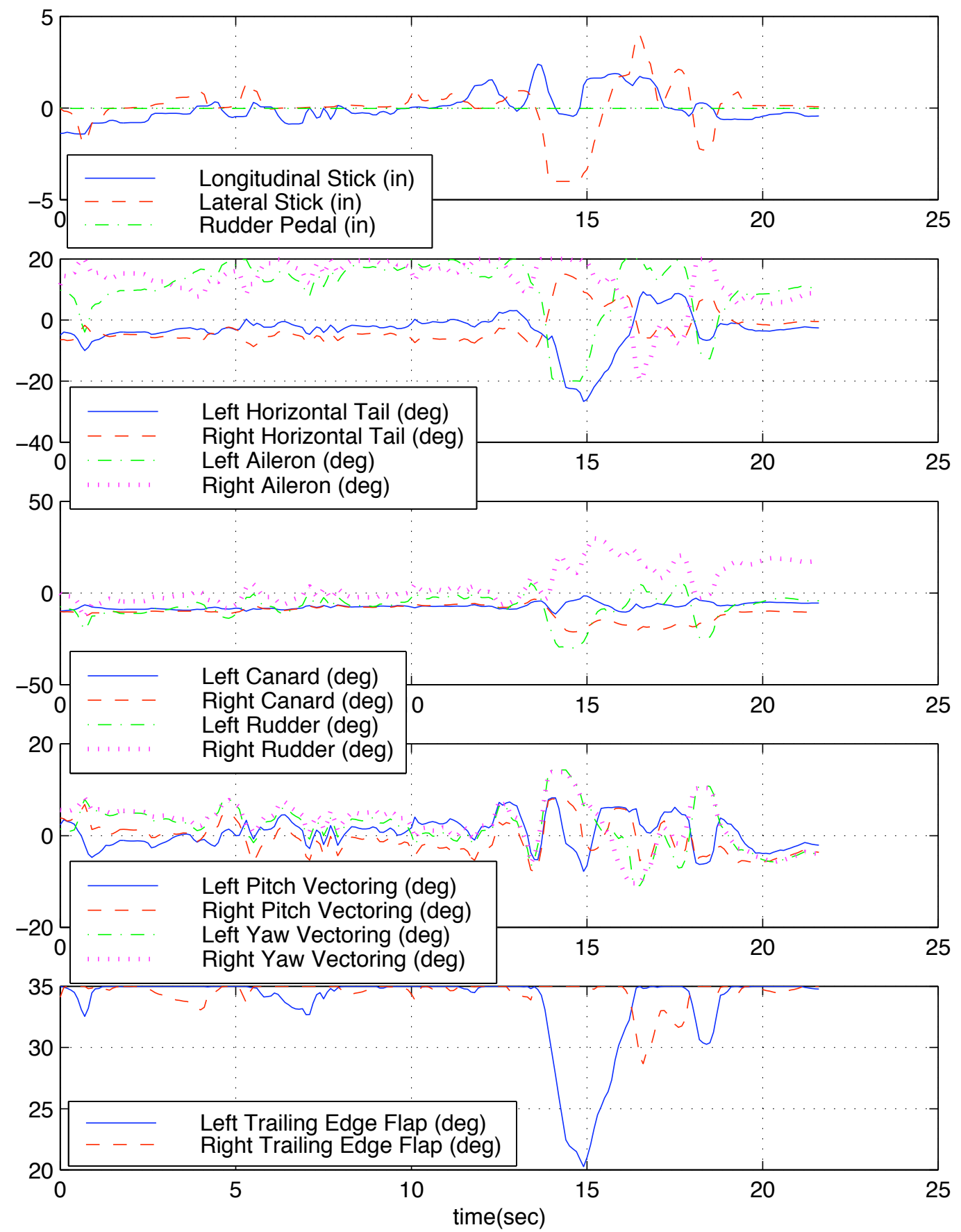

Figure 6.14: Control effector deflections during terrain following task with no failures. 

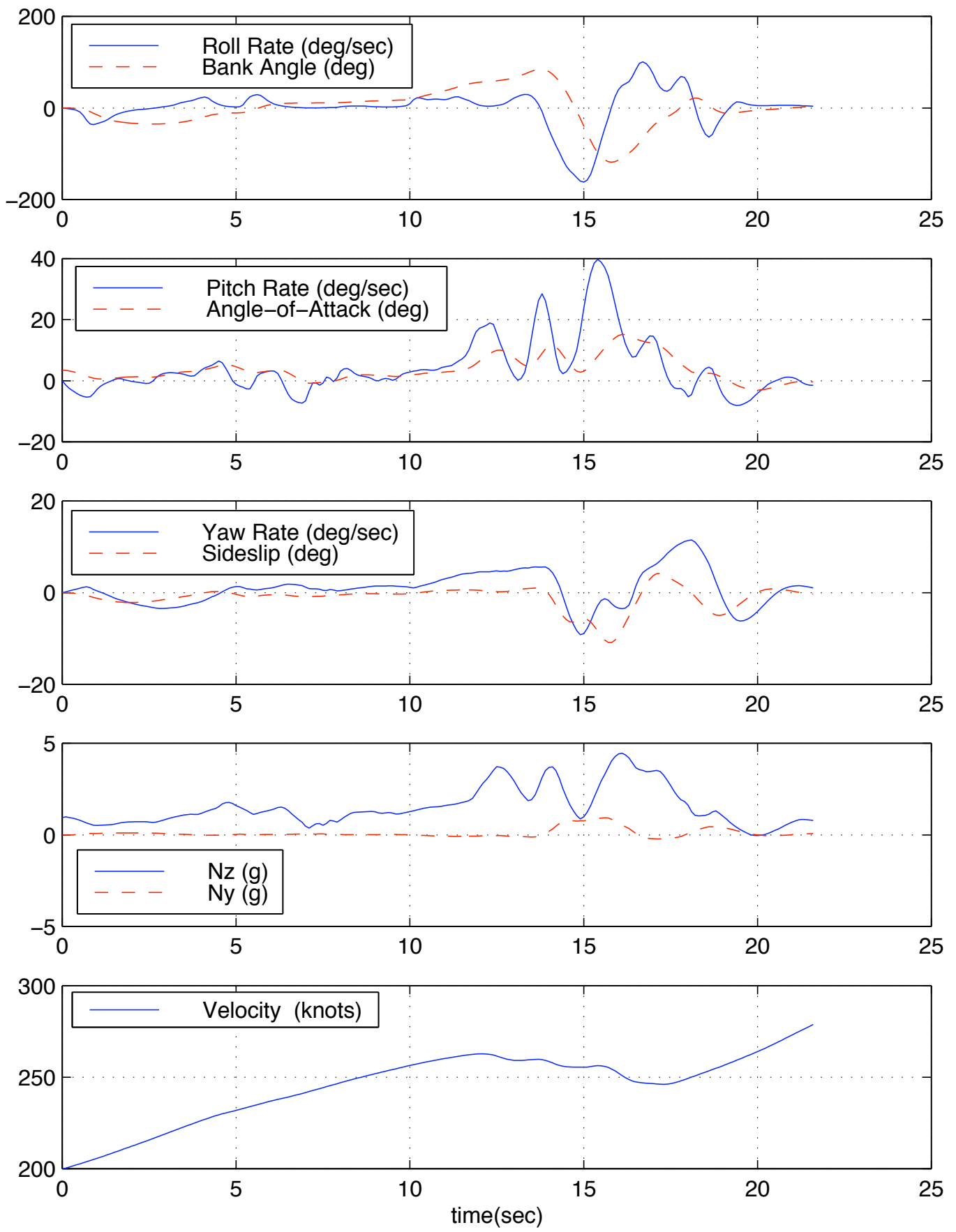

Figure 6.15: Aircraft states during terrain following task with no failures. 


\section{Chapter 7}

\section{Control Reconfiguration}

\subsection{Background}

The goal of reconfigurable flight control systems is to adaptively use the remaining undamaged control surfaces to compensate for failures and damages to the aircraft. This type of system has the potential to dramatically increase the survivability of an aircraft. Reconfigurable flight control systems allow the aircraft to maintain maneuverability after the advent of a failure. The survivability of the aircraft after a failure is a function of the type of failure that occurs as well as the number of redundant control effectors remaining after the failure. In a classical aircraft configuration that utilizes only a horizontal tail, a pair of differential ailerons and a single rudder, the loss of any one of these controls can prove to be catastrophic. However, in an aircraft such as the F-15 ACTIVE with its 12 independent control surfaces, the loss of a single control can easily be accommodated for by the reconfigurable control system through the use of the remaining 11 controls. The remaining controls have the capability to not only eliminate the adverse effects due to the failed control, but also, allow the aircraft to maintain a level of performance that would enable the pilot to successfully complete the mission at hand. This paper will demonstrate the capability of MRA on the F-15 ACTIVE to maintain acceptable performance after a combination of control failures.

The use of reconfigurable control systems offers not only savings in long term survivability of an aircraft, but also can lower the initial production cost of an aircraft. In most aircraft, redundant hydraulic systems exist to provide back-up for failed systems. Reconfigurable flight control systems could potentially reduce the need for this level of redundancy. In turn, 
this reduction in back-up hardware systems can lower the weight and ultimately the cost of an aircraft. Although the necessary level of acceptance of reconfigurable flight control systems into the aircraft community to eliminate mechanical back-up systems presently does not exist, the potential for this acceptance is plausible.

It should be noted that control reconfiguration is only one portion of a completely adaptive control system. Unfortunately, it is the second step in the accommodation of a failure. The first step is the identification of the failure. Recent research has sought methods of determining when a failure occurs and what form the failure has taken. [21] Control effector failures can vary in spectrum from an aileron stuck at its stop to the complete loss of a horizontal tail. The identification of these failures is a non-trivial practice that is beyond the scope of this paper.

Although the potential exists to store a set of data for subsets of predetermined failures, the idea becomes unrealistic when one considers the large number of combinations and types of control failures. Consider the situation in which a control surface is damaged by enemy aircraft fire or surface to air missile. If the effector sustains damage in the form of a large hole in the center of the surface, a position sensor at the hinge of the surface would not be sufficient to identify the failure. In fact, what has occurred in reality is a column in the control effectiveness matrix, $\mathbf{B}$, has been modified. The identification of this change in control effectiveness must be calculated real-time.

In the remainder of the work discussed in this paper, it is assumed that the control failure has been successfully identified. Only the reconfiguration of the control system in response to the identified failure is investigated. Section 7.2 will give information about how failure reconfiguration is implemented in conjunction with the Moment Rate Allocator with the Bisecting, Edge-Searching Algorithm. Then, section 7.3 will give the reader a few example responses of the F-15 ACTIVE during control failure reconfiguration situations.

\subsection{Implementation}

The implementation of control failure reconfiguration in the F-15 ACTIVE using MRA with BESA is a straightforward procedure. First, as stated above, it is assumed that the failure has been identified. The failure examples investigated in this research are therefore considered to be known a priori by the algorithm. In the implementation, several "pre-determined" 
control failures were coded into the simulation. The term "pre-determined" is placed in quotations to emphasize that its definition does not imply the storage of pre-calculated control effectiveness matrices for each failure.

Once the failure has been identified, the first step in the reconfiguration process is to eliminate the column or columns of the $\mathbf{B}$ matrix associated with the failed control surface or control surfaces from the problem. This modification of the $\mathbf{B}$ matrix is done by changing the indices of the remaining components of the $\mathbf{B}$ matrix and essentially compacting the columns to produce $\mathbf{B}^{3 \times m-r}$, where $r$ is the number of failed controls. In addition, if control restoring is performed utilizing minimum-drag, minimum-sideforce or any other objective function that necessitates the use of the control surfaces' effects on the objective, ie. $\frac{\partial \mathbf{y}}{\partial \mathbf{u}}$, the indices of these components of the 4 th row of the augmented $\mathbf{B}$ matrix must also be reorganized to produce $\mathbf{B}_{A u g}^{4 \times m-r}$.

The desired change in moment, $\Delta \mathbf{m}_{d}$, must recognize the failed control effectors' contribution to moments actually attained by the aircraft. In the event of a jamming of a control surface at one of its stops, its resulting effect on the overall moment generated on the aircraft can be found by multiplying its column of control effectiveness by the position at which it is jammed. Stated more generally, when calculating the moments attained in the previous frame, $k-1$, one must make sure to use the entire control effectiveness matrix and all of the current control positions.

$$
\mathbf{m}_{\text {attained }}=\mathbf{B}_{\text {full }} \mathbf{u}_{\text {full }}
$$

where $\mathbf{m}_{\text {attained }}=\mathbf{m}_{k-1}$. The commanded change in moment is simply the previous moment attained subtracted from the new moment commanded.

$$
\Delta \mathbf{m}_{d_{k}}=\mathbf{m}_{d_{k}}-\mathbf{m}_{k-1}
$$

The frame-wise control allocation is then performed utilizing the reduced set of control effectors and the desired change in moment, $\Delta \mathbf{m}_{d_{k}}$.

The type of failure that is associated with a change in the values of the components of one or more of the columns of the $\mathbf{B}$ matrix is not investigated in this paper. This problem is not discussed for two primary reasons. First, it is difficult to determine a reasonable modification to the control effectiveness of a surface caused by adding a "hole" to the surface. Second, 
once one has identified that change in effectiveness, using a real-time identification algorithm, the new effectiveness data can be inserted into the original column or columns associated with the failed control surface or surfaces. The allocation problem then remains of the same dimension as the original un-failed problem with only a change in the magnitudes of components of the $\mathbf{B}$ matrix associated with the failed controls.

\subsection{Examples}

In order to add some reality to the failure conditions simulated in this work, it was decided that the failures chosen for investigation would be representative of actual failures on an aircraft. The two primary failures investigated in this work are an aileron stuck at its stop and the loss of both the left and right horizontal tails. The aileron failure is designed to demonstrate the allocator's ability to reconfigure and eliminate adverse effects from control effectors jammed in a non-symmetric configuration. The double horizontal tail failure is included to exhibit the allocator's capability to reallocate control surfaces to accommodate the loss of primary pitch and roll generating surfaces. Each of these failures could feasibly occur in an actual aircraft based on the typical layout of an aircraft hydraulic system.

The representative maneuvers discussed in chapter 6 are used for evaluation of the aircraft's performance in the control failure reconfiguration mode. In the investigation of the real-time maneuvers, the failures to the aircraft were part of the aircraft's initial condition. However, it is possible to implement a failure that occurs at some point during the maneuver. Trial runs with the failure inserted in the middle of the maneuver did not reveal any significant aircraft performance degradation compared to those runs in which the aircraft started the run with the failure already incorporated.

The sections that follow will evaluate the allocator's performance in failure reconfiguration during several representative maneuvers. It will be shown that although there was a significant change in the attainable moment subset of the aircraft, the aircraft was still in general able to complete the mission tasks and satisfy the desired performance criteria. Note that the F-15 ACTIVE was designed with a large amount of control power. Due to this excessive control power, control reconfiguration is possible. (ie. the F-15 ACTIVE, derived from a pre-production F-15 with only 8 control effectors, does not require 14 independent control effectors in order to achieve acceptable flying qualities ratings) 
Although data is not presented in this paper for lateral offset powered approaches to an aircraft carrier in a control failure configuration, this maneuver was investigated. Evaluation of this landing task did not produce any interesting results. In fact, the simulated control failures were transparent to the pilot during the task. In addition, it was determined that the level of lateral offset required to produce the desired level of task difficulty did not represent a realistic powered approach situation.

\subsubsection{Lateral Stick Doublet: Aileron Failure}

The roll rate response of the aircraft to a canned lateral stick doublet is included at this point to show the degradation in rolling moment capability due to the aileron failure. With a right aileron jammed at its positive 20 degree stop, the aircraft response to a 4 inch lateral stick doublet at 400 knots and 10,000 ft did not exhibit the smooth first order response in roll rate that was evident in the non-failure mode. However, it was determined that the rolling moment required or commanded by the full lateral stick doublet was not attainable with the available control power. As discussed earlier in this paper, if a commanded moment is outside the AMS, the best the allocator can do is utilize the full capabilities of the control effectors to attain the largest possible moment in the same direction as the commanded moment. This commanding of a moment outside the AMS is exactly what happened in the failed aileron lateral stick doublet that was investigated. The aircraft response to lateral stick doublets of 4, 3 and 2 inches in magnitudes are shown in Figure 7.1.

As will be shown in Table 7.2, the failure of the right aileron limits the aircraft to only $45 \%$ of its original positive rolling moment generation capability. Investigation into a 3 inch lateral stick doublet at the same flight condition revealed that the aircraft - allocator combination was very close to achieving the commanded moment. In response to a 2 inch lateral stick doublet, the failure of the right aileron had no significant effect on aircraft response. Since the gearing on the lateral stick is somewhat linear, (ie. full lateral stick corresponds to maximum roll rate and half lateral stick deflection corresponds to about half maximum roll rate), a 2 inch lateral stick doublet is within the $45 \%$ of maximum rolling moment capability available during the aileron failure. Note that in all three cases evaluated, the aircraft's negative roll rate response was identical for the failed and non-failed configurations. From this investigation of the lateral stick doublet one can conclude that no matter how optimal your allocator is, an allocator can not, under any circumstance, produce 


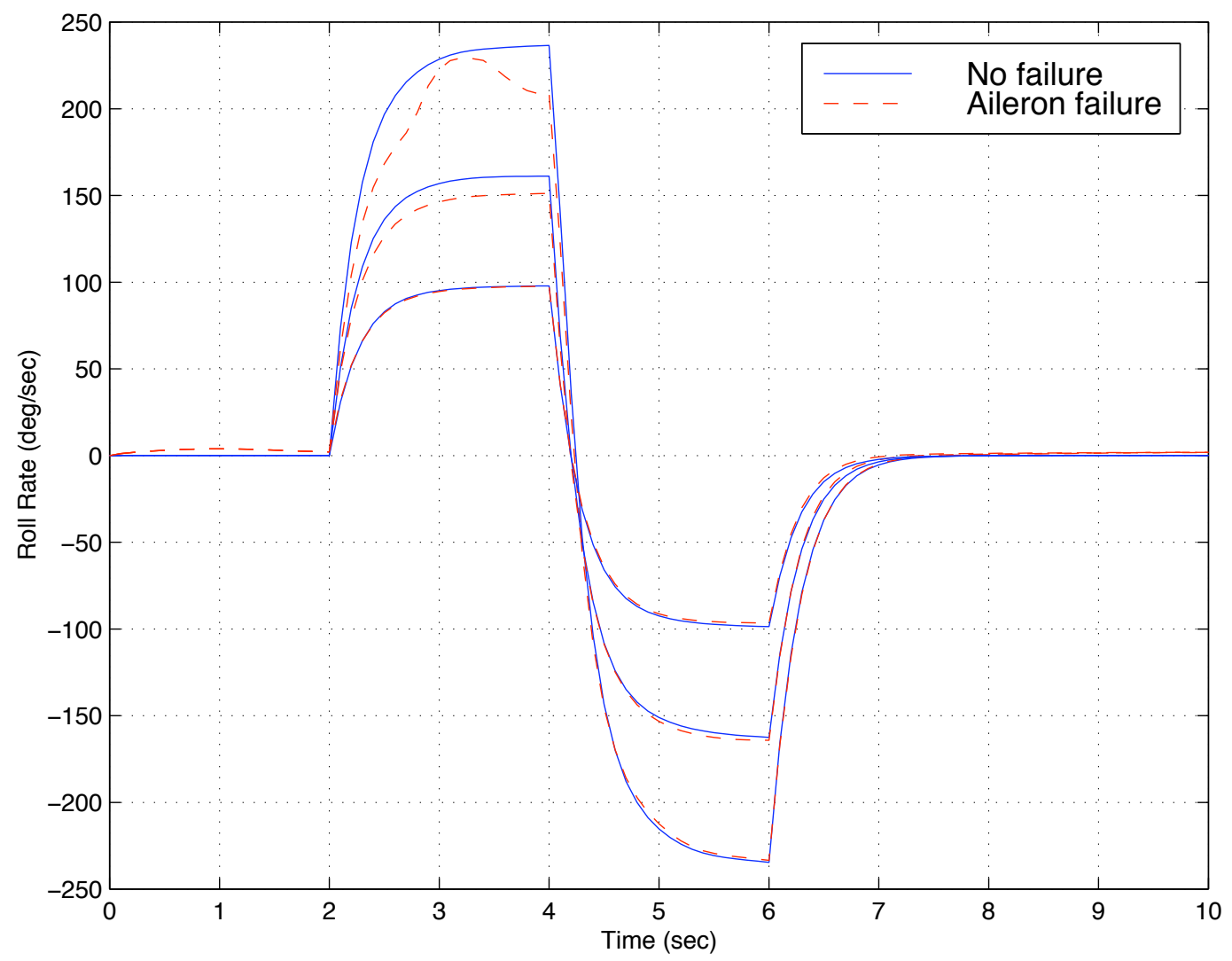

Figure 7.1: Lateral stick doublet with minimum-norm restoring with/without a right aileron failure. 4, 3 and 2 inch lateral stick doublets shown for the F-15 ACTIVE at 400 knots and $10,000 \mathrm{ft}$.

a control combination that generates a larger moment than is defined by the AMS.

\subsubsection{High Yo-Yo: Aileron Failure}

The theory behind the High Yo-Yo maneuver was discussed in section 6.2. However, to refresh the reader, the High Yo-Yo maneuver is used in air combat maneuvering to eliminate excess closure rate on a bogey and attain a tracking solution on the bogey. The desired and adequate performance criteria was defined in Table 6.1. The desired performance was to place the bogey inside the smaller circle of the pipper with a 25 second time limit.

A graphical depiction of the attainable moment subset of the F-15 ACTIVE at 400 knots and 10,000 ft is shown in Figure 7.2. In this figure, the facets of AMS associated with the right aileron have been highlighted in a light gray color in order to distinguish them from 
the remainder of the facets. The remainder of the colors assigned to the facets of the AMS are arbitrary. In the jamming of the right aileron at a positive 20 degree stop, one essentially collapses this band of facets to which the right aileron contributes. If one were to rotate around the entire AMS, the facets associated with the right aileron would form a continuous band. In fact, each control surface can be associated with a band of facets that encircles the AMS. From a geometric perspective, this continuous band (or zone) gives rise to the name zonotope. [22]

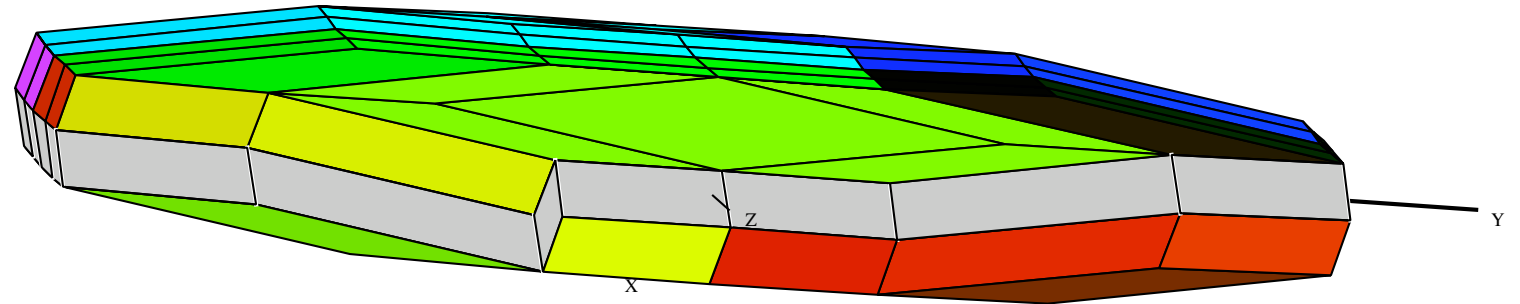

Figure 7.2: Right aileron highlighted in gray to show contribution to AMS. F-15 ACTIVE at 400 knots and 10,000 ft.

Table 7.1 lists the minimum and maximum attainable pure rolling, pitching and yawing moments for the F-15 ACTIVE at a flight condition of 400 knots and 10,000 ft. In this discussion, minimum moment refers to the maximum moment in the negative direction. These numbers will be used for comparison purposes in the evaluation of the loss of moment capability during a failure mode. A control allocation toolbox (CAT) was utilized to generate the graphical depictions of the AMS and to calculate the minimum/maximum moments along the three respective moment axes. The CAT software is capable of calculating maximum attainable moments in any direction, but the interpretation of maximum moment in an arbitrary direction does not offer any insight into the AMS unless one knows that a given maneuver will demand a moment in that direction.

Table 7.1: Minimum/maximum attainable moments for the F-15 ACTIVE with 12 controls at 400 knots and 10,000 ft.

\begin{tabular}{|l|c|c|}
\hline Moment & Minimum Moment & Maximum Moment \\
\hline \hline Roll & -0.07903 & 0.07902 \\
\hline Pitch & -0.51480 & 0.54375 \\
\hline Yaw & -0.10823 & 0.10826 \\
\hline
\end{tabular}

Using the CAT software, one can set a control surface at any particular value and evaluate 
the new AMS associated with the remaining controls. Figure 7.3 shows the AMS after the jamming of the right aileron at its positive 20 degree stop. Recall in Figure 7.2 that the facets associated with the right aileron were highlighted in light gray. In Figure 7.3 it is clear that the AMS is identical to that in Figure 7.2 with the highlighted light gray facets removed.

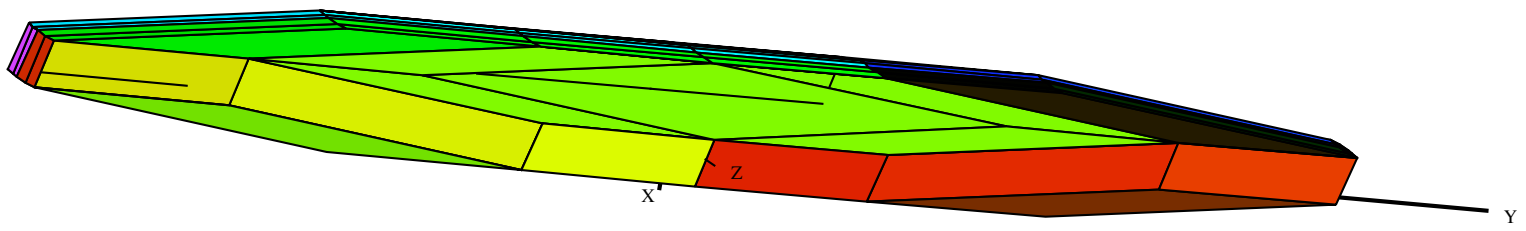

Figure 7.3: AMS after right aileron hard-over to +20 degrees. F-15 ACTIVE at 400 knots and $10,000 \mathrm{ft}$.

To give an analytical interpretation of the change in size of the AMS, Table 7.2 lists the minimum and maximum attainable pure rolling, pitching and yawing moments of the F15 ACTIVE at 400 knots and 10,000 $\mathrm{ft}$ with the right aileron jammed at its positive 20 degree stop. The table also lists the corresponding percentages of the original non-failure mode attainable moments. There is some degradation in the pitching and yawing moment capability of the aircraft due to the failure of the right aileron, but it is clear from the table that the primary moment generating loss occurs in the aircraft's capability to produce positive rolling moment. However, this loss of positive rolling moment makes complete intuitive sense. If the right aileron is stuck at its positive 20 degree stop, there is no potential for utilizing this aileron to generate a positive, right wing down, rolling moment. During a right wing down rolling moment command, one must utilize the differential capabilities of the remaining control effectors to produce the desired moment. In addition, note that with the right aileron jammed at it's positive 20 degree stop the aircraft can still achieve $100 \%$ of its original negative rolling moment. Therefore, as intuition leads one to believe, the maximum negative rolling moment capability, (ie. the largest right wing up moment), occurs when the right aileron is at its positive 20 degree stop.

To reinforce the statements made at the end of section 7.3.1, recall the 4 inch lateral stick doublet. Degradation to approximately $45 \%$ of the aircraft's original rolling moment capability explains the inability of the aircraft to attain the full lateral stick command. To reiterate, a control allocator cannot produce a control combination that generates an aircraft moment that is not attainable. 
Table 7.2: Minimum/maximum attainable moments for the F-15 ACTIVE with 12 controls at 400 knots and 10,000 ft. Right aileron jammed at the +20 degree stop.

\begin{tabular}{|l|c|c|c|c|}
\hline Moment & Min. Moment & \% Min. Moment* & Max. Moment & \% Max. Moment* \\
\hline \hline Roll & -0.07903 & 100.0 & 0.03623 & 45.8 \\
\hline Pitch & -0.51050 & 99.2 & 0.52218 & 96.0 \\
\hline Yaw & -0.09668 & 89.3 & 0.10825 & 100.0 \\
\hline
\end{tabular}

* Percentage of original moment without control failure.

Results from the High Yo-Yo maneuver with the right aileron failure using MRA with minimum-norm restoring are shown in figures 7.4 through 7.6. This trial was flown by subject pilot A. Although the maneuver was flown many times with the failure implemented, only one run is included in this paper. In general, during all the runs attempted, the failure was transparent to the pilot. This transparency is to be expected during this maneuver since the maneuver is primarily a longitudinal maneuver and hence there is limited use of differential control surface commands. However, the data is included to demonstrate the transparency of the control failure reconfiguration to the pilot.

The first subplot of Figure 7.4 shows the flight path trajectories of the attacker and bogey during the High Yo-Yo maneuver. It is clear from the bearing and range plots of this figure that the pilot was able to complete the mission in the desired amount of time. The pilot's bearing and range to the bogey were well within the desired criteria by the 25 second mark. Control inceptor deflections shown in the first subplot of Figure 7.5 show that although there are some lateral stick oscillations, the primary input into the system is a longitudinal command. As discussed in section 6.2.4, excessive lateral stick oscillations were a nonintentional part of the piloting technique during the maneuver. Subplot two of Figure 7.5 shows the horizontal tail and aileron deflections during the maneuver. From this plot note that the right aileron, shown as a dashed magenta line, is constant at positive 20 degrees.

As a result of the minimum-norm restoring that was implemented in this trial, the restoring algorithms have attempted to eliminate as much of the differential deflections as possible while still attaining the desired moments. The horizontal tails remained in differential mode opposite that of the ailerons throughout the maneuver to counteract the rolling moment generated by the jammed aileron. The use of the horizontal tails for the elimination of the 
adverse rolling moment caused by the failed aileron is expected because the horizontal tails have a high effectiveness in roll in comparison to the remaining control surfaces.

The aircraft states shown in Figure 7.6 are similar to those that were found in the non-failure mode evaluation of the High Yo-Yo in section 6.2.4. The lateral stick input at about the 2 second mark causes the spike in the roll rate curve found in the first subplot at 2 seconds. There is a positive roll rate spike that occurs immediately after this initial lateral stick input. It is believed that this roll rate spike is the pilot's attempt to stop the aircraft's roll rate and hold the aircraft in a banked turn for the remainder of the maneuver.

Pitch rate and angle-of-attack are relatively constant after the initial pull up in the maneuver. From the third subplot of Figure 7.6 it is clear that the aileron failure had no significant effect on the regulation of sideslip. A doublet in yaw rate is present around 2 seconds corresponding to the lateral stick doublet. In this maneuver, it appears that the pilot has placed himself in a higher $g$ turn throughout the maneuver than was found previously in the non-failure mode. However, comparing the flight path trajectories of Figure 7.4 and 6.7, the failed and non-failed configurations, one sees that in the failed configuration the pilot has chosen to attempt to track inside the bogey's banked turn after he returns to the bogey's altitude of 10,000 ft. Without the failure, the pilot chose to slightly overshoot the bogey's path and then track him slightly outside his trajectory. This difference in piloting technique explains the difference in normal acceleration between the two runs. Nevertheless, this difference in piloting technique does not seem to have any direct link to the failure. The pilot was not informed before each flight whether or not his aircraft had any failures. Therefore, the pilot was blind to the configuration that he was flying and thus could not intentionally tailor or modify his piloting technique to accommodate for the control failure. 

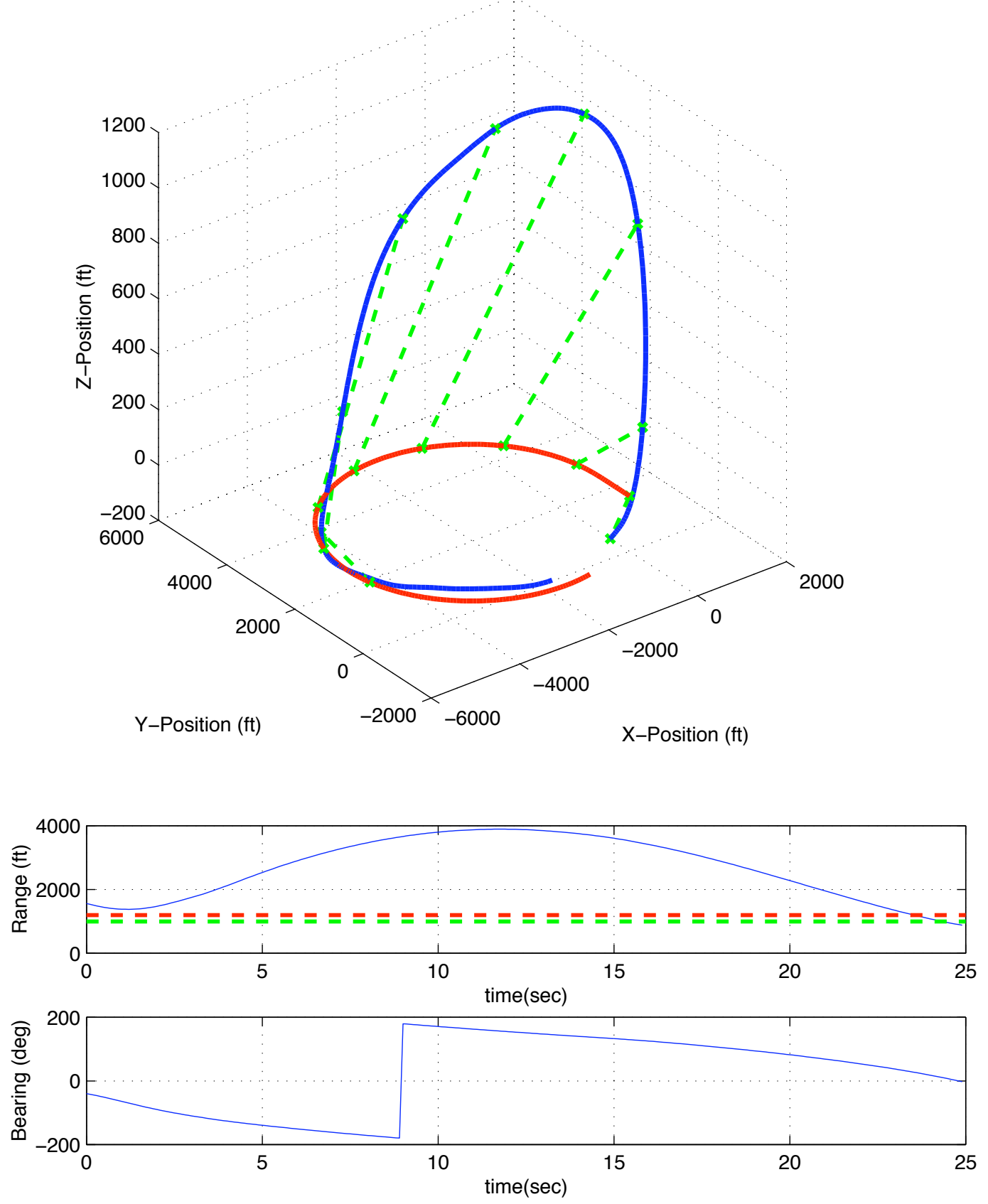

Figure 7.4: Time history of attacker and bogey during High Yo-Yo maneuver with right aileron failure. Bearing and range shown throughout the maneuver. Dashed red and green lines indicate adequate and desired ranges, respectively. 


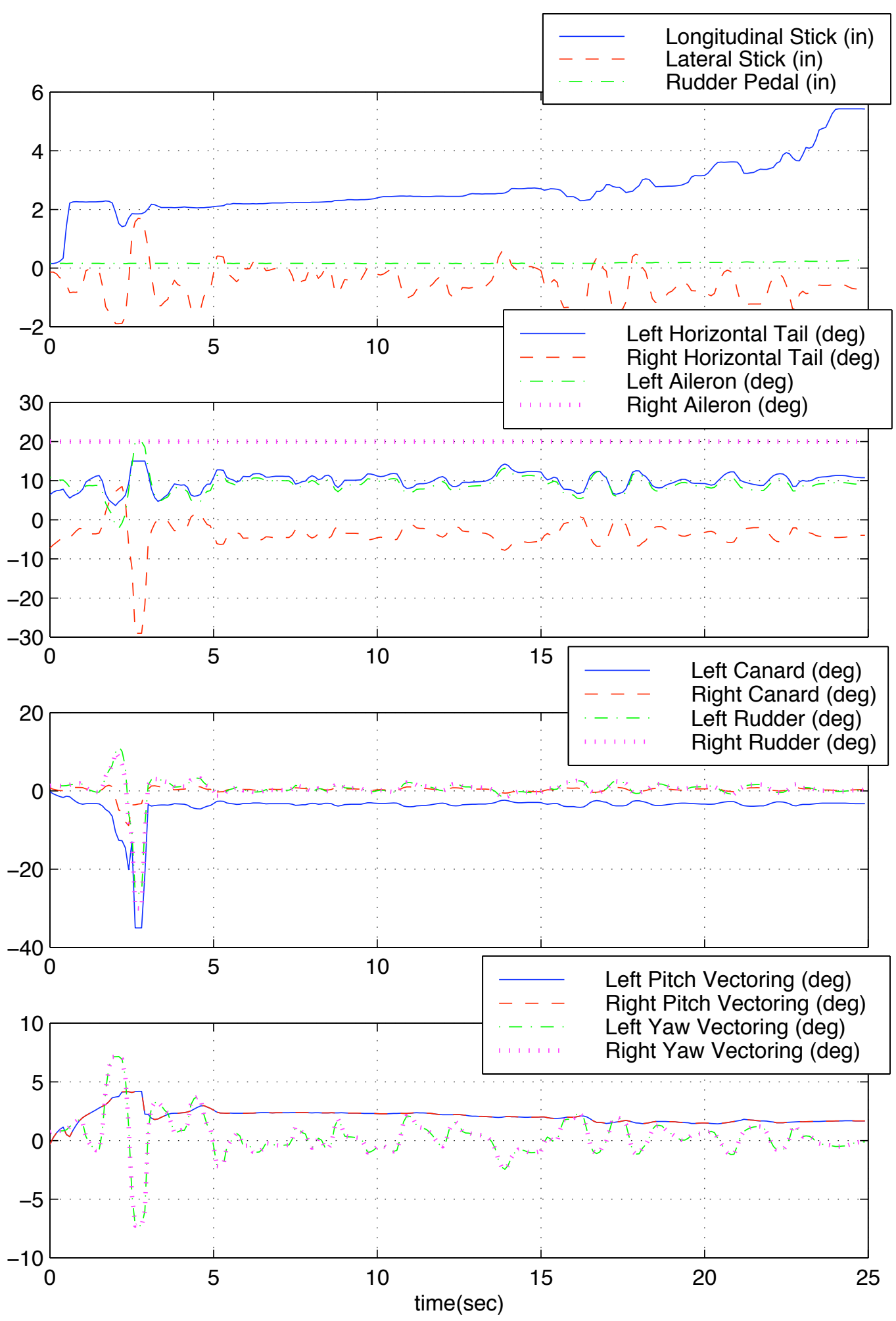

Figure 7.5: Time history of control effector positions during High Yo-Yo maneuver with right aileron failure. Minimum-norm restoring implemented. 

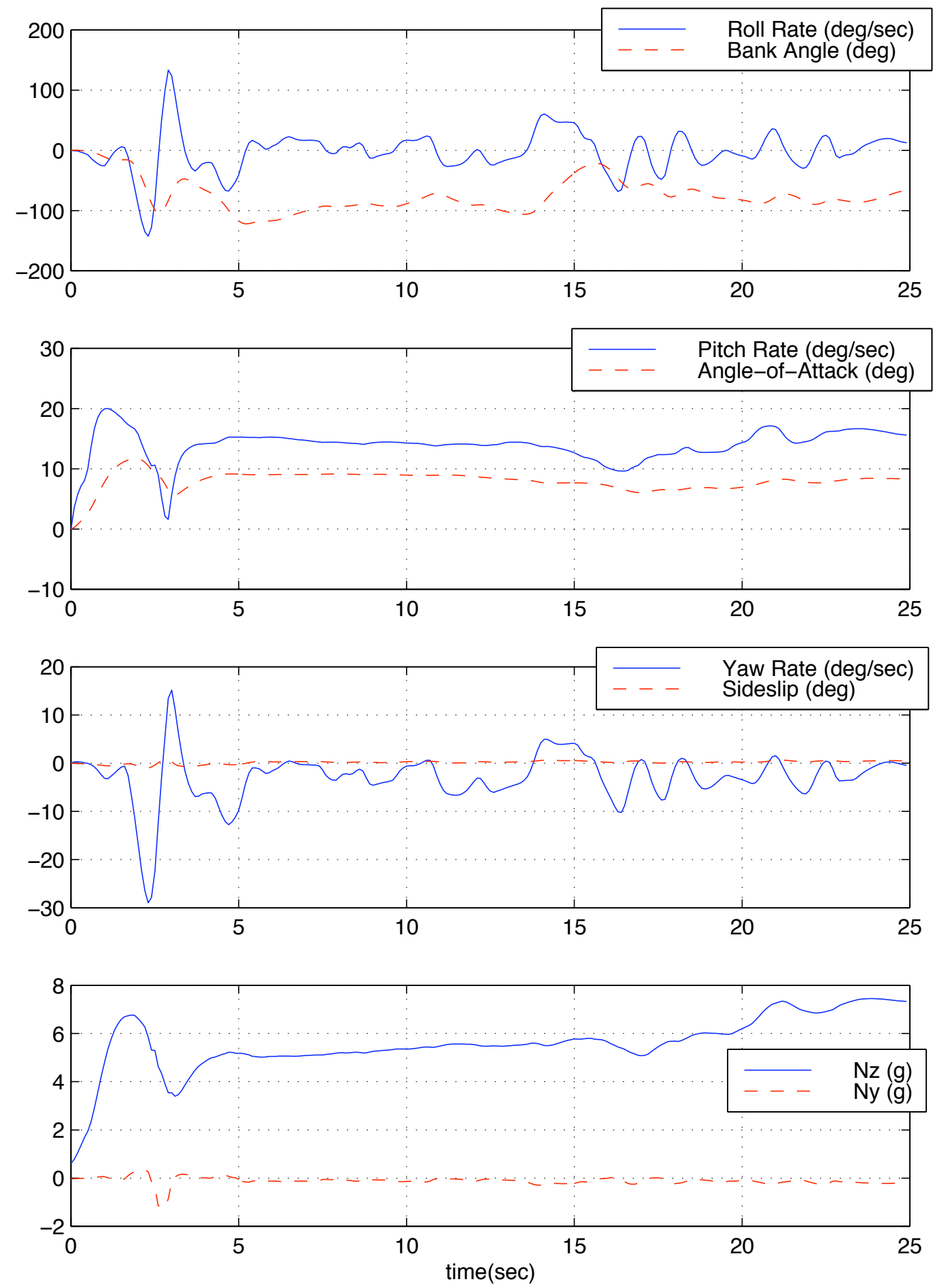

Figure 7.6: Time history of aircraft states during High Yo-Yo maneuver with right aileron failure. Minimum-norm restoring implemented. 


\subsubsection{High Yo-Yo: Double Horizontal Tail Failure}

Since the High Yo-Yo was determined to be primarily a longitudinal maneuver, a control failure that limited the longitudinal performance capabilities of the aircraft was sought. A failure of both horizontal tails was implemented. Table 7.3 shows the volume of the AMS for the un-failed case, the aileron failure and the double horizontal tail failure. Corresponding percentages of the original volume of the un-failed configuration are given in the third column of the table. Note that while the right aileron failure of section 7.3.2 produced an AMS that was still $67.7 \%$ of the original volume, the double horizontal tail failure reduced the volume to only $33.6 \%$ of the original value.

Table 7.3: AMS volume with and without failed controls.

\begin{tabular}{|l|c|c|}
\hline Failure Type & AMS Volume & Percentage of Original AMS \\
\hline \hline No Failure & $2.67680 \mathrm{e}-2$ & 100 \\
\hline Right Aileron $(+20 \mathrm{deg})$ & $1.81266 \mathrm{e}-2$ & 67.7 \\
\hline Left \& Right Horizontal Tails $(+0 \mathrm{deg})$ & $8.99894 \mathrm{e}-3$ & 33.6 \\
\hline
\end{tabular}

Figure 7.7 shows the AMS for the F-15 ACTIVE at a flight condition of 400 knots and 10,000 $f t$. The facets that are associated with the left and right horizontal tails are highlighted in gray to show their contributions to the AMS. Again, as in section 7.3.2, the failure of these controls essentially eliminates them from the surface of the AMS.

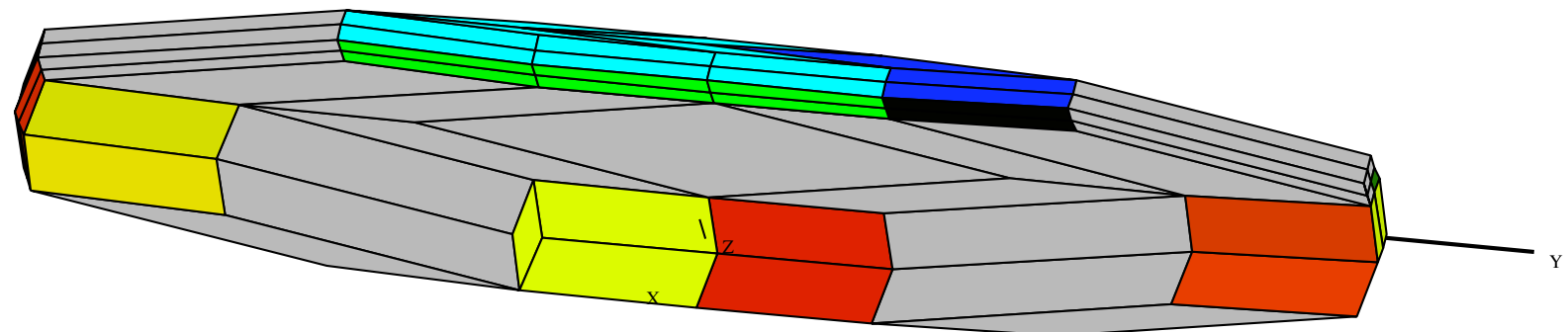

Figure 7.7: Facets associated with left and right horizontal tails highlighted in gray to show contribution to AMS. F-15 ACTIVE at 400 knots and 10,000 ft.

After the removal of the facets associated with the left and right horizontal tails, the size of the AMS has been significantly reduced. Figure 7.8 shows the AMS for the F-15 ACTIVE at the same flight condition as above, but with both horizontal tails jammed at the zero degree position. The reader should note that the scale for Figure 7.8 is identical to that of 
Figure 7.7. Clearly the size of the AMS after the failure would lead one to believe that there would be a significant reduction in aircraft performance. The completion of the High Yo-Yo in this failed configuration disproves this theory.

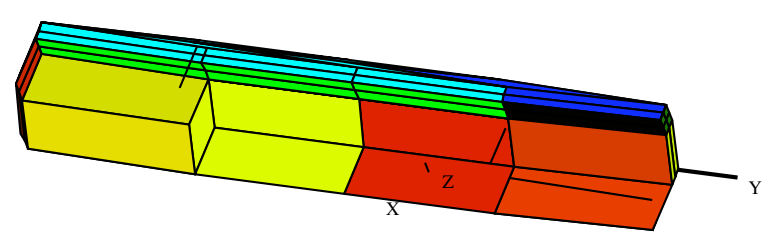

Figure 7.8: AMS after left and right horizontal tail jammed at 0 degrees. F-15 ACTIVE at 400 knots and 10,000 ft.

The most significant reduction in attainable moments due to the double horizontal tail failure occurs in the positive pitching moment capability of the aircraft. In fact, if one studies Table 7.4 they will see that the aircraft maintains only $44.4 \%$ of its original positive pitching moment capability and only $69.5 \%$ of its negative pitch moment capability after the onset of the failure. The reduction in positive and negative rolling moment capability found in Table 7.4 reiterates the high effectiveness of the horizontal tails in generating rolling moments. Furthermore, since this failure is symmetric in nature, the reduction in rolling and yawing moments is identical for the positive and negative cases of each respective moment.

Table 7.4: Minimum/maximum attainable moments for the F-15 ACTIVE with 12 controls at 400 knots and 10,000 ft. Left and right horizontal tail jammed at 0 degrees.

\begin{tabular}{|l|c|c|c|c|}
\hline Moment & Min. Moment & \% Min. Moment* & Max. Moment & \% Max. Moment* \\
\hline \hline Roll & -0.04633 & 58.6 & 0.04629 & 58.6 \\
\hline Pitch & -0.35768 & 69.5 & 0.23999 & 44.1 \\
\hline Yaw & -0.09307 & 86.0 & 0.09308 & 86.0 \\
\hline
\end{tabular}

* Percentage of original moment without control failure.

Results from the High Yo-Yo maneuver with the double horizontal tail failure are shown in figures 7.9 through 7.11. For the data presented, the aircraft was in a minimum-norm restoring configuration. The pilot for these results was subject pilot A. Figure 7.9 shows the trajectories of the attacker and bogey during the maneuver. The bearing and range subplots of Figure 7.9 clearly indicate that the pilot was able to attain the required tracking 
solution within the desired time. In fact, the pilot accomplishes the range portion of the task about 2.5 seconds before the set desired time limit. From these plots, one can conclude that the pilot was able to accomplish the mission with the double horizontal tail failure without significant degradation in performance of the aircraft.

The pilot workload during the maneuver with the double horizontal tail failure was not significantly different from the un-failed configuration. The plot of the time histories of control inceptor inputs is found in the first subplot of Figure 7.10. A large aft longitudinal stick input was initiated at approximately the 1 second mark. A period of sustained longitudinal stick input is evident. The drop off in longitudinal stick input between the times of 10 and 15 seconds can be attributed to the desire of the pilot to avoid the onset of the previously mentioned aircraft departure. Lateral stick oscillations occur throughout the maneuver. These oscillations did not have a significant effect on the aircraft performance. In fact, when asked for a Cooper-Harper Handling Qualities Rating [23], the pilot responded with, "HQR of 1 or 2, easiest flight yet". It should be reiterated that the pilot was purposely not informed of his aircraft configuration before each flight to eliminate the possibility of his modifying piloting technique for the failure. As in all previous discussions of the High Yo-Yo, the pilot again has opted not to utilize his rudder pedals during the maneuver.

The horizontal tail and aileron deflections during the maneuver are plotted in the second subplot of Figure 7.10. Note that both horizontal tails remain at their zero positions throughout the entire time history. Larger differential aileron deflections are used in this maneuver than were utilized in the un-failed case of Figure 6.8. This increase in differential ailerons is a direct result of the elimination of the horizontal tails and their contributions to rolling moment in the differential configuration.

An increased amount of rudder deflection is evident in the third subplot of Figure 7.10. This increase is most likely due to the loss of the horizontal tails' ability to generate a yawing moment. The canard deflections shown in the third subplot indicate that the canards are used primarily in a symmetric configuration. The magnitude of the canard deflection is not significantly different from that of the un-failed case in Figure 6.8. However, a less negative canard deflection during the maneuver produces less nose down pitching moment on the aircraft and essentially accommodates for the loss of nose up pitching moment due to the horizontal tails that is no longer present.

The pitch and yaw thrust vectoring time histories show the expected symmetric responses. 
A positive pitch vectoring deflection is maintained throughout the maneuver contributing to the aircraft nose down pitching moment. Oscillations in the yaw thrust vectoring result from the corresponding oscillations in lateral stick inputs.

The aircraft states are shown in Figure 7.11. The pilot attempted to maintain approximately a 90 degree bank angle throughout the maneuver. Oscillations in roll rate are found in the first subplot of Figure 7.11. These oscillations are caused by the pilot's lateral stick inputs. The pilot held an approximately 20 degree/second pitch rate during the initial pull-up of the aircraft and into the banked turn. At about the 10 second mark, he reduced his longitudinal input and as a result his pitch rate dropped to about 5 degrees/second. At the end of the task, from about 15 seconds until 25 seconds, the pilot increased his longitudinal stick input and his pitch rate again rose. This portion of the maneuver corresponds to the point at which the pilot realized he had dropped below the bogey's altitude and took corrective action to attain his tracking solution.

The third subplot of Figure 7.11 shows that although variations in yaw rate of positive 5 degrees/second to -12.5 degrees/second occurred, the sideslip angle was well regulated. Lateral acceleration, plotted in the fourth subplot, was also kept to a minimum. The normal acceleration during the task was held at about $6 g^{\prime} s$ initially, dropped to about $3 g^{\prime} s$ in the 10 to 15 second time frame and then returned to $6 \mathrm{~g}^{\prime} s$ at about the 20 second mark. This level of load factor was typical for the pilot during his multiple runs of the task.

It is clear that even with the reduction in AMS volume to $33.6 \%$ of the original volume, the pilot was able to accomplish the High Yo-Yo task. Furthermore, the pilot did not complain about any loss of aircraft performance during the maneuver with the double horizontal tail failure. In fact, as stated before, the data included in this section was from a run that the pilot stated produced his easiest tracking solution. The pilot's comment should not be interpreted as saying that the aircraft is easier to fly with the loss of the left and right horizontal tails. Instead, this section has shown that no significant degradation in aircraft performance occurs due to the loss of a set of primary pitch generating effectors. The reconfiguration capabilities of MRA have performed superbly and made the control failure transparent to the pilot during the task. This excellent performance is indicative of the potential benefits of utilizing MRA on tactical aircraft with multiply redundant control effectors in air combat maneuvering situations in which the possibility of completely losing or jamming control surfaces due to battle damage is high. 

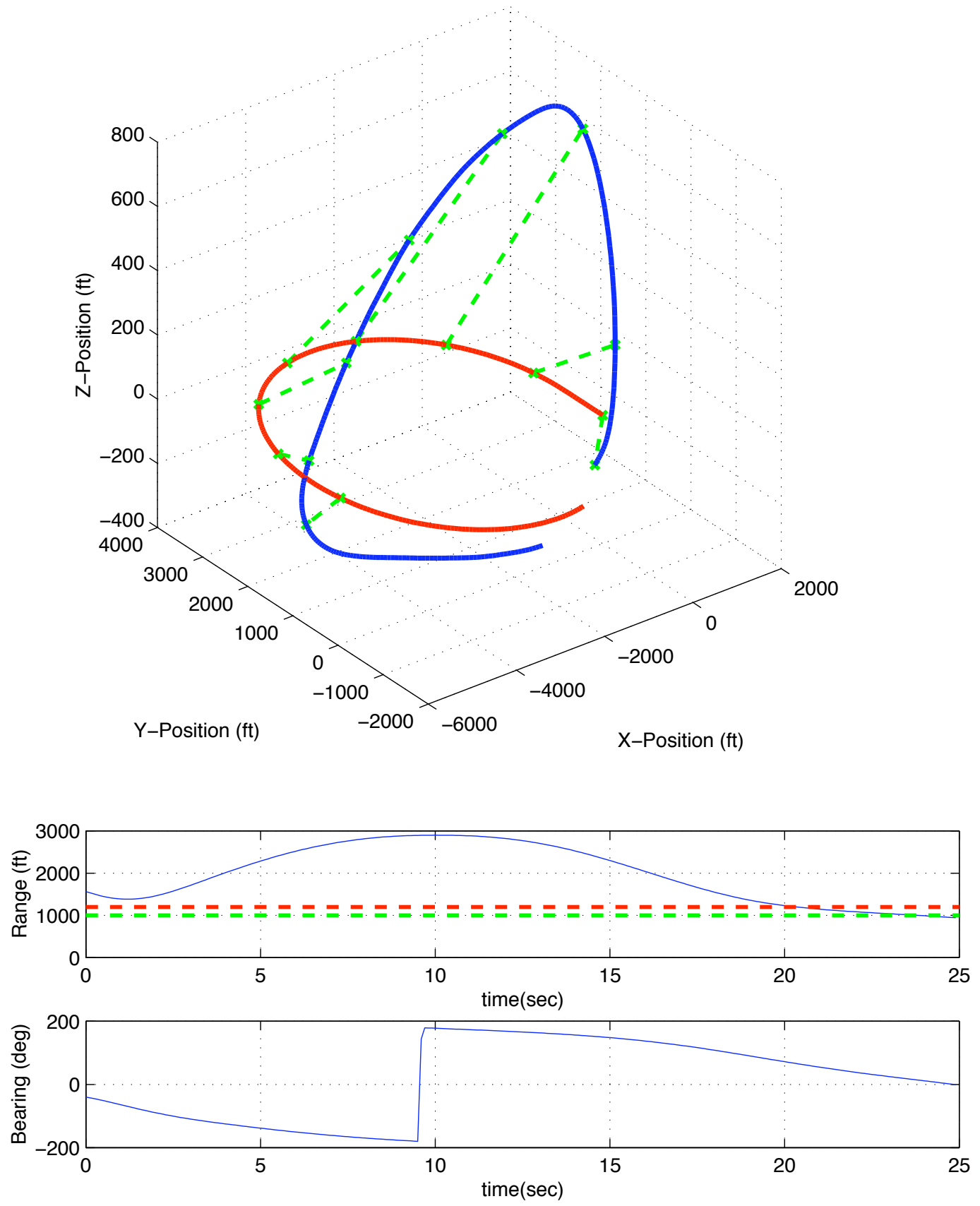

Figure 7.9: Time history of attacker and bogey during High Yo-Yo maneuver with double horizontal tail failure. Bearing and range shown throughout the maneuver. Dashed red and green lines indicate adequate and desired ranges, respectively. 


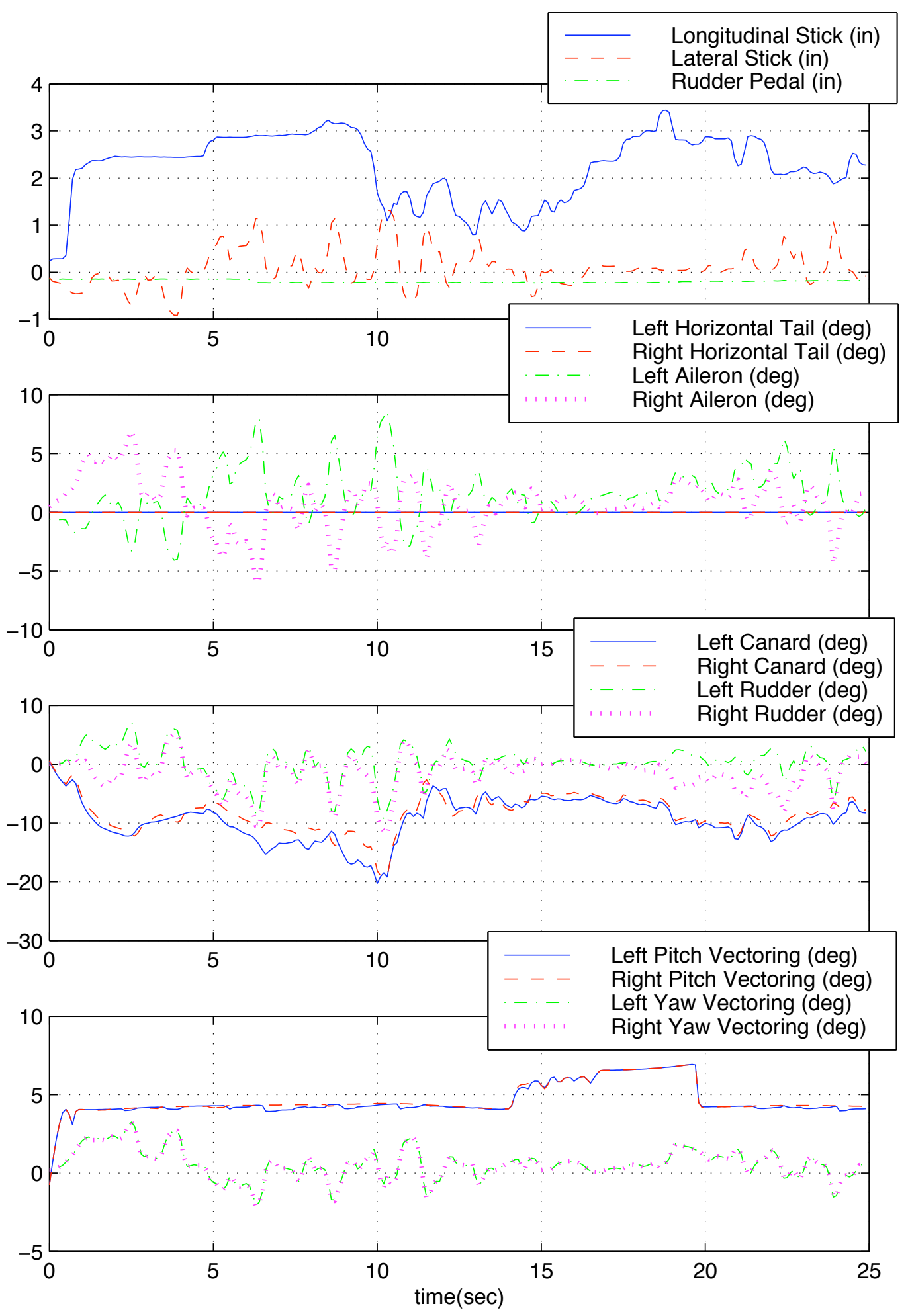

Figure 7.10: Time history of control effector positions during High Yo-Yo maneuver with double horizontal tail failure. 

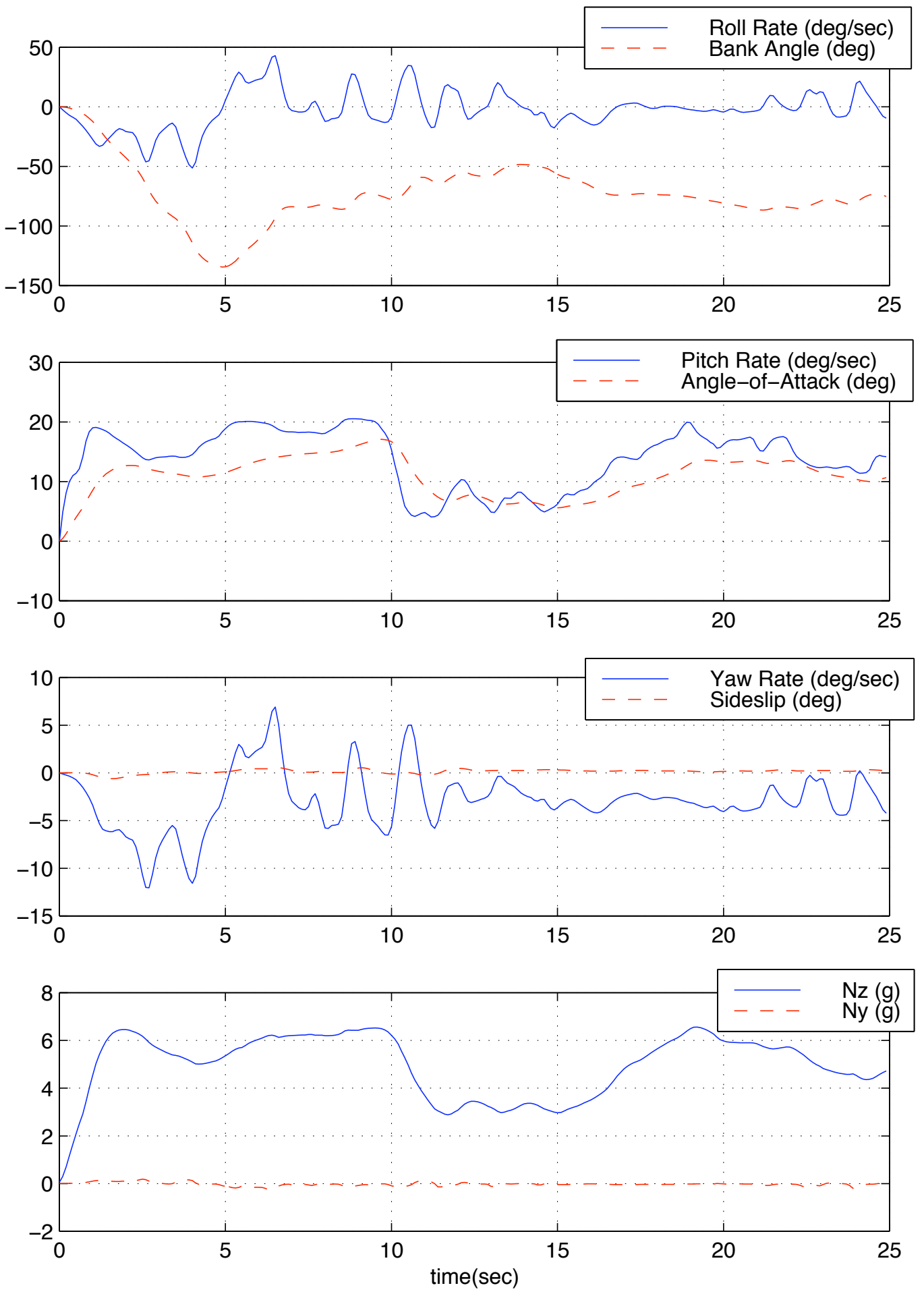

Figure 7.11: Time history of aircraft states during High Yo-Yo maneuver with double horizontal tail failure. 


\subsubsection{Terrain Following: Double Horizontal Tail Failure}

The terrain following task was flown in several control failure configurations. The data presented in this section were taken from a flight flown by subject pilot B with the F15 ACTIVE in a flaps-down configuration with a failure of both horizontal tails to their zero degree positions. Ailerons and flaps were drooped to 20 degrees in this configuration. Figure 7.12 shows the AMS for the F-15 ACTIVE at $200 \mathrm{knots}$ and $200 \mathrm{ft}$ in the flaps-down configuration. The facets that correspond to the left and right horizontal tails have been highlighted in gray to show their contribution to the AMS.

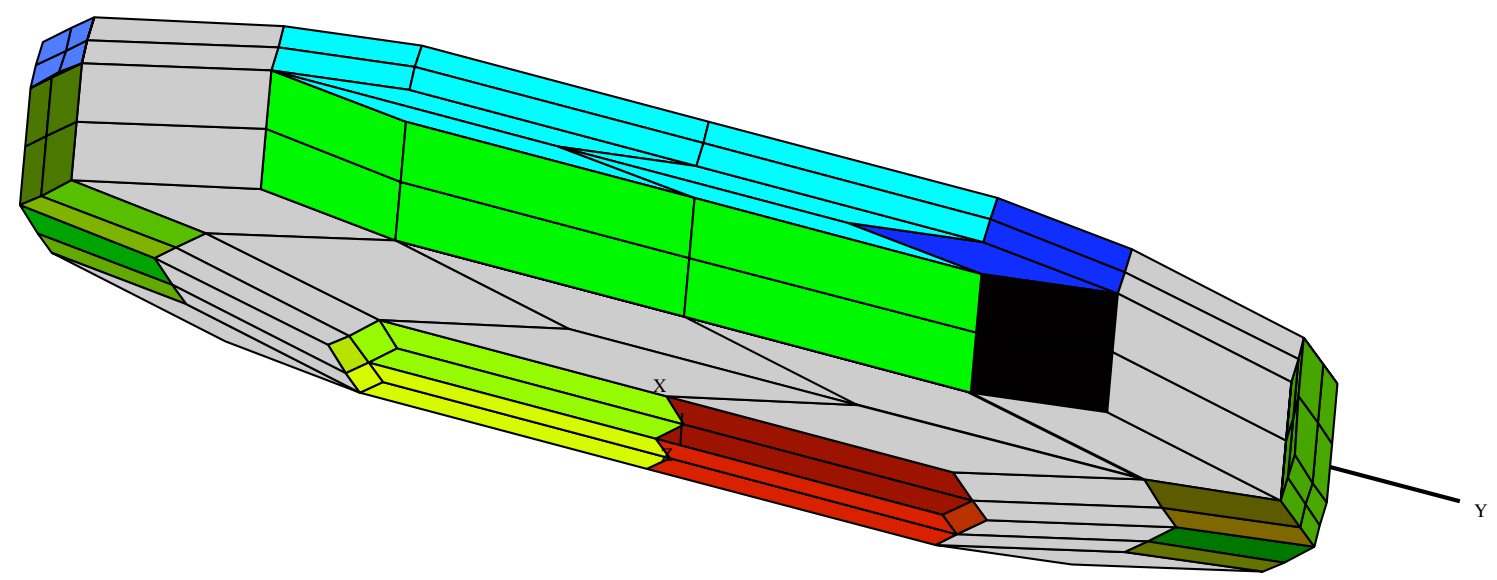

Figure 7.12: Left and right horizontal tails highlighted in gray to show contribution to AMS. F-15 ACTIVE at 200 knots and $200 \mathrm{ft}$, flaps-down configuration.

The AMS for the F-15 ACTIVE in the powered approach configuration with the failure of the horizontal tails is illustrated in Figure 7.13. As before, it is clear from this figure that the failure of the horizontal tails results in the removal of the facets associated with them from the AMS. The volume of the AMS before their removal was 1.90412e-1 units ${ }^{3}$. Since non-dimensional moment coefficients were used in the generation of the AMS, although the volume dimension is listed as units ${ }^{3}$, it is actually non-dimensional units ${ }^{3}$. However, after the failure, the AMS has a volume of only $8.01156 e-2$ units $^{3}, 42.1 \%$ of the original volume.

As a result of the loss of the horizontal tails, the maximum moment generating capabilities of the aircraft have changed. Table 7.5 lists the maximum and negative and positive moment capabilities of the aircraft after the failure. The capabilities before the failure are not listed, but the percentage of pre-failure capabilities is given. Note that the most significant effect of losing the horizontal tails was a degradation in rolling moment. There was a reduction 


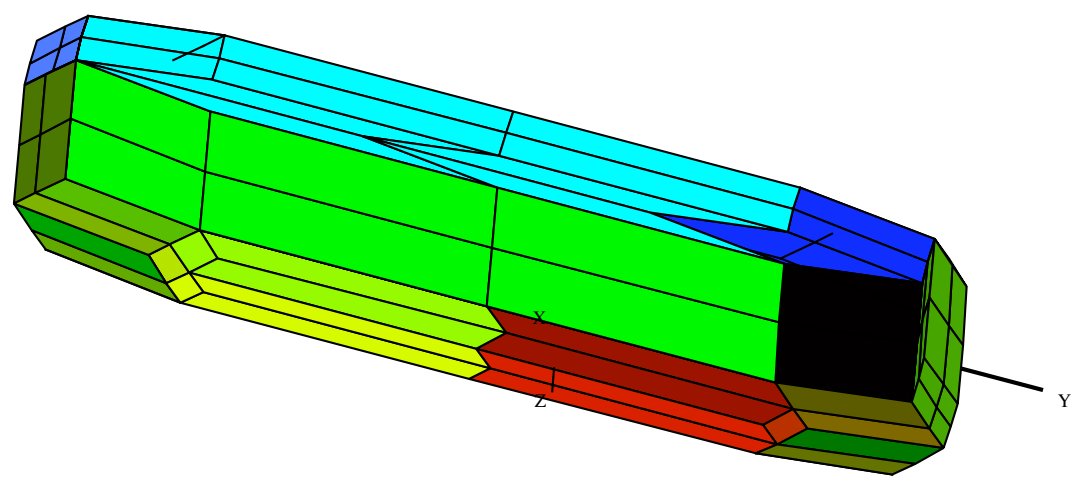

Figure 7.13: AMS after left and right horizontal tails jammed at 0 degrees. F-15 ACTIVE at 200 knots and $200 \mathrm{ft}$, flaps-down configuration.

to only $62.1 \%$ of the original positive pitching moment that has a significant impact on the aircraft's ability to pitch nose-up. This loss of nose-up pitching moment and rolling moment capabilities was very detrimental during the terrain following task.

Table 7.5: Minimum/maximum attainable moments for the F-15 ACTIVE with 12 controls at 200 knots and $200 \mathrm{ft}$, flaps-down configuration. Left and right horizontal tails jammed at 0 degrees.

\begin{tabular}{|l|c|c|c|c|}
\hline Moment & Min. Moment & \% Min. Moment* & Max. Moment & \% Max. Moment* \\
\hline \hline Roll & -0.04491 & 58.2 & 0.04487 & 58.2 \\
\hline Pitch & -0.62953 & 81.3 & 0.46013 & 62.1 \\
\hline Yaw & -0.46421 & 91.5 & 0.46421 & 91.5 \\
\hline
\end{tabular}

* Percentage of original moment without control failure.

It was found to be very difficult for the pilots to maneuver through the terrain following task with control failures. Although the success rate without failures was only about $25 \%$, with control failures, the task could only be completed about $15 \%$ of the time. However, in the cases that the pilot was able to complete the task, the data obtained was extremely interesting. Figures 7.14 through 7.16 are taken from a terrain following flight of subject pilot B with the double horizontal tail failure. From Figure 7.14 it appears that the pilot was able to maneuver the aircraft through the buildings along the desired trajectory. However, close observation of the aerial view subplot of Figure 7.14 shows that the pilot passed directly through the second red building. This data is representative of one of the "best" flights with 
the failures.
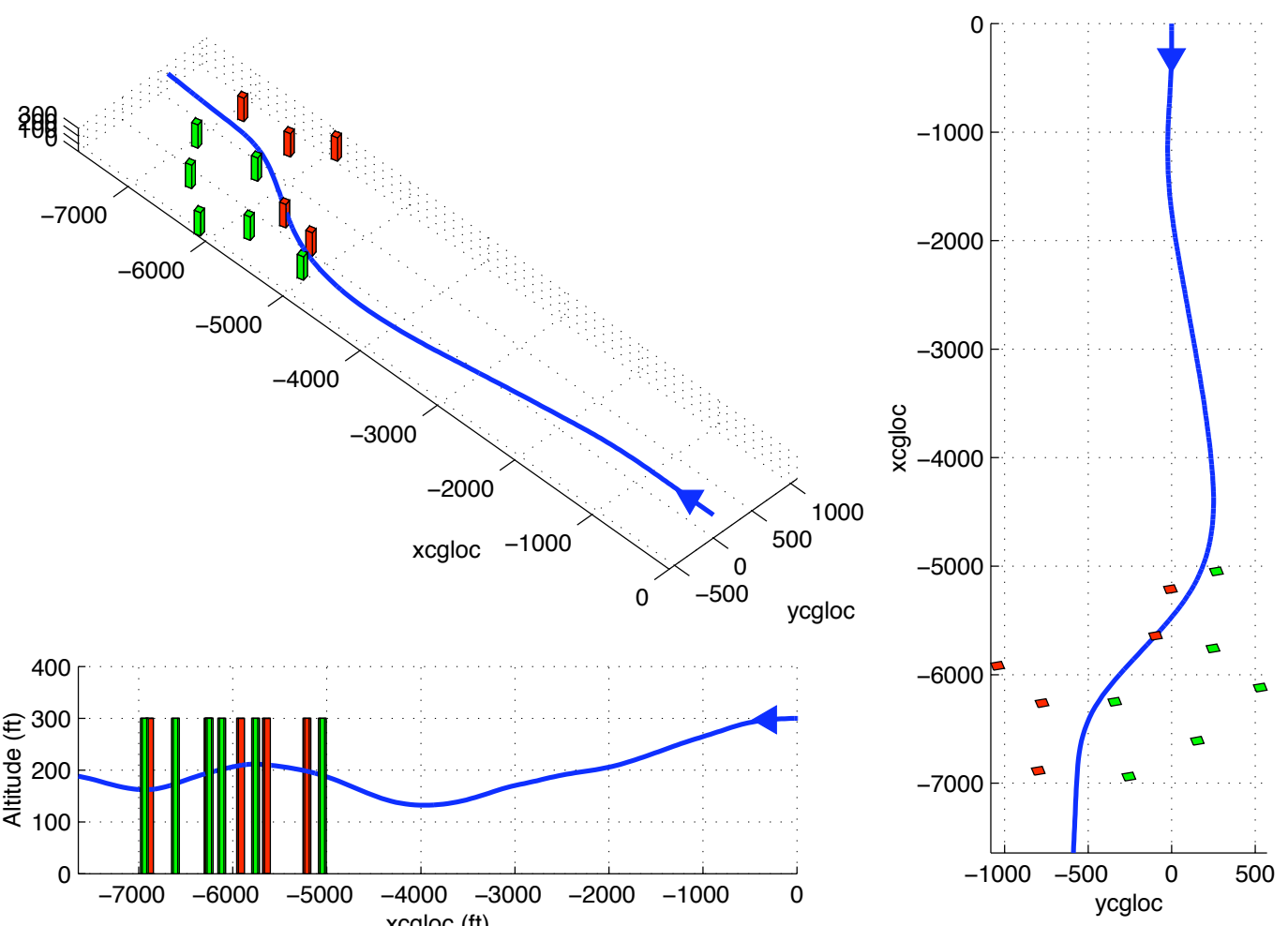

Figure 7.14: Trajectory and altitude during terrain following task with double horizontal tail failure.

Pilot stick inputs are shown in the first subplot of Figure 7.15. Large lateral stick and longitudinal stick deflections occur from the 12 second time to the completion of the maneuver. This portion of the data occurs as the pilot is flying between the buildings. The lateral stick deflection is similar in shape to a step doublet. As illustrated in the second subplot, the left and right horizontal tails remain at their zero deflection point throughout the duration of the task. Significant position saturation of the ailerons is evident in the second subplot. The large lateral stick inputs produced rolling moment commands that demanded the use of full differential aileron deflection. Due to the loss of the horizontal tails, little control power was available to allow the ailerons to restore towards their desired droop deflection of 20 degrees.

Canard and rudder deflections for the maneuver are shown in the third subplot of Figure 7.15. Large differential rudder and canard deflections occur while the pilot is maneuvering through the buildings. The symmetric differential rudder deflection appears to be attempting to help generate positive pitching moment. Thrust vectoring time histories are given in the fourth 
subplot. These deflections are in general symmetric.

Of interest is the flap deflection shown in the fifth and final subplot of Figure 7.15. As mentioned in section 6.3.3, the saturation of the flaps seen in this subplot is an anomaly that the author was not able to conclusively resolve. It is thought that the problem arose due to peculiarities in the attained control effectiveness data. In addition, as explained in section 6.3.2, it is believed that the restoring algorithm used to droop the ailerons and flaps, although mathematically sound, demanded far too much from the aircraft.

In the high pilot workload portion of the task, a significant differential flap deflection occurs. The low 18 degree/second rate limit on the left flap is evident in the time history of its deflection. Between the 12 and 15 second times, the left flap follows a saw tooth trajectory that is often associated with rate limiting. This limit on flap deflection rate was noticed by the pilot during the task. The roll rate of the aircraft appeared to lag behind the pilots command forcing the pilot to attempt to lead the aircraft during the maneuver. In attempting to lead the aircraft, the pilot points his aircraft's velocity vector at a location in front of the bogey. In most cases that the task was not successfully completed, the lack of success was attributed to the aircraft not having enough rolling moment generating capability to allow the pilot to bank the aircraft through the second portion of the s-turn trajectory. It should be noted that this observation occurred in both the failure and non-failure configurations.

Time histories of the aircraft's states during terrain following task are shown in Figure 7.16. The roll rate and bank angle are found in the first subplot. The bank angle plot indicates that the pilot placed the aircraft into a banked turn before entering the building area. This allowed him to lead the aircraft through the first turn. Bank angles of \pm 100 degrees occurred during the maneuver. Note that the maximum roll rate achieved was only about 150 degrees/second, only about $65 \%$ of the 230 degrees/second maximum roll rate capability for the aircraft in a flaps up configuration at $400 \mathrm{knots}$ and 10,000 ft. This maximum roll rate occurs at approximately the 13 second mark, the point at which the pilot has full lateral stick deflection.

Large oscillations in pitch rate are illustrated in the second subplot of Figure 7.16. These oscillations were result of the bank and pull technique utilized by the pilot. In addition, piloting technique was modified to prevent the onset of large angles-of-attack and the associated aircraft departure from controlled flight. The angle-of-attack remained relatively benign during the task. However, many of the non-successful flights of this task were ended 
due to large angles-of-attack and the previously mentioned departure.

Yaw rate varied between \pm 10 degrees/second during the maneuver while the sideslip angle was regulated between \pm 5 degrees. Lateral acceleration was close to zero throughout the maneuver. Peaks in normal acceleration of approximately 5 and $7 \mathrm{~g}$ 's occurred as the pilot performed two successive bank and pull turns. The $7 g$ turn is representative of subject pilot B's aggressive pilot technique that does not account for excessive accelerations and their effects on a pilot in an actual aircraft. Full afterburner was commanded as the pilot entered the buildings in order to maintain speed during the task. The plot of velocity with respect to the wind shown in Figure 7.16 shows that the pilot attempted to gain airspeed during the maneuver in order to increase his maneuvering potential and allow him to complete the task.

This section has shown that the terrain following task could be completed with a double horizontal tail failure. Although the data presented in this section indicated a collision with the second red building, runs were completed in this failed configuration that did not involve any collisions. Unlike the High Yo-Yo task, the failure was not transparent to the pilot. There was a significant increase in pilot workload during the task with the control failure and an associated decrease in success rate. Despite this, one must consider the demands that the pilot is placing on the aircraft in attempting to complete the mission. The task was found to be extremely difficult to complete even in the un-failed mode. In general it was the opinion of all pilots who flew the aircraft that the powered approach configuration was more difficult to fly than the flaps-up configuration. The aircraft is far more controllable at higher speeds and altitudes than it is with the flaps-down at slow speeds. However, this increased controllability at higher velocities is typical of tactical aircraft. The low dynamic pressure flight regime produces slower dynamic responses in the aircraft and as a consequence tasks such as the terrain following task investigated in this research become more difficult to complete.

The primary purpose for attempting the terrain following task in the failed configuration was to demonstrate that the aircraft is not only controllable with the failure, but also still perform the task. The fact that the task was completed, even if only $15 \%$ of the time, indicates that the F-15 ACTIVE with MRA and a double horizontal tail failure maintains satisfactory maneuvering capability in the failed configuration. Although data was not included in this work for powered approach to a field or carrier, the aircraft was able to repeatedly land successfully in a control failure mode. The ability to land in a control failure configuration 
has the potential to increase the survivability of damaged aircraft and also prevents the potentially dangerous need for a pilot to eject from his aircraft over enemy territory. 

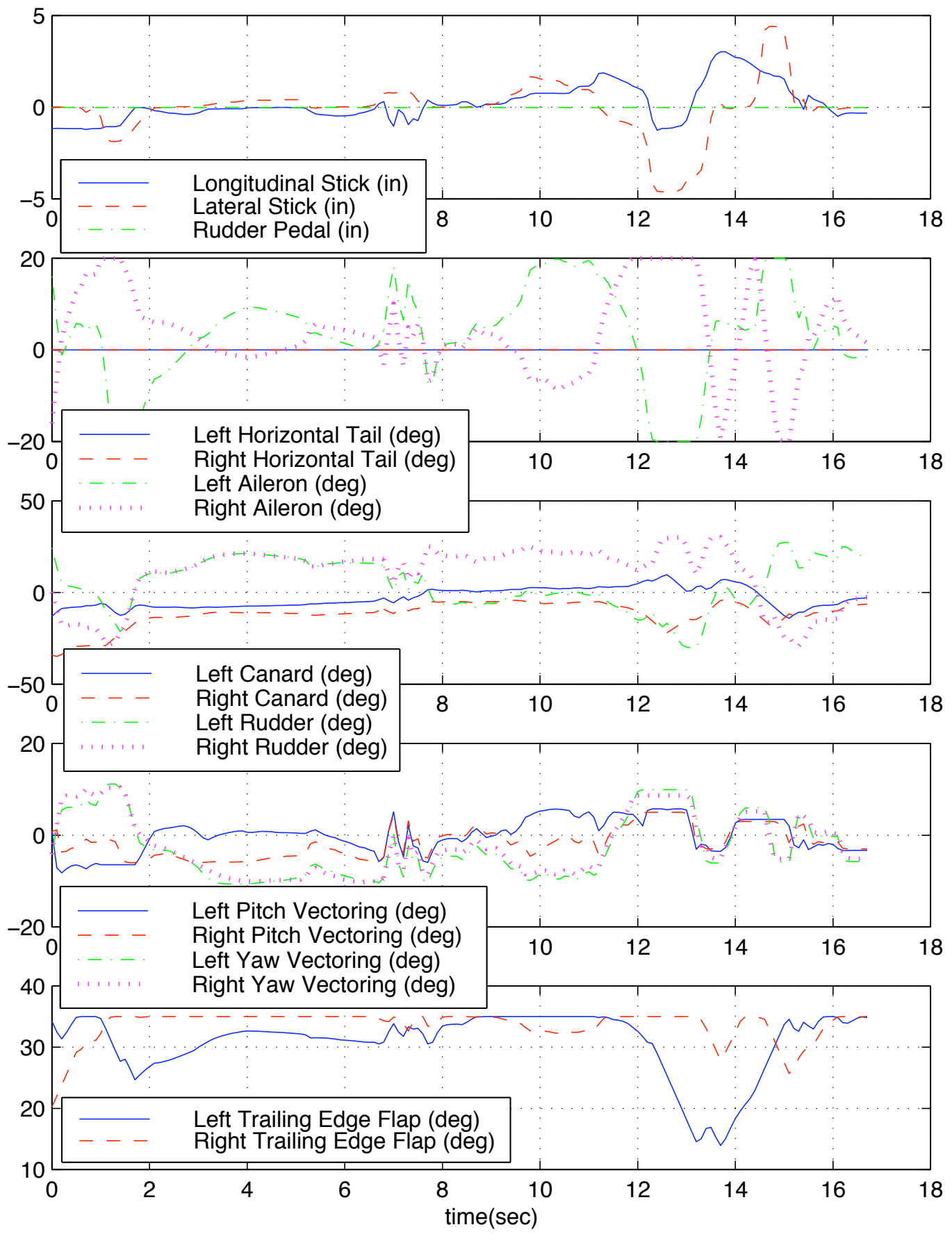

Figure 7.15: Control effector deflections during terrain following task with double horizontal tail failure. 

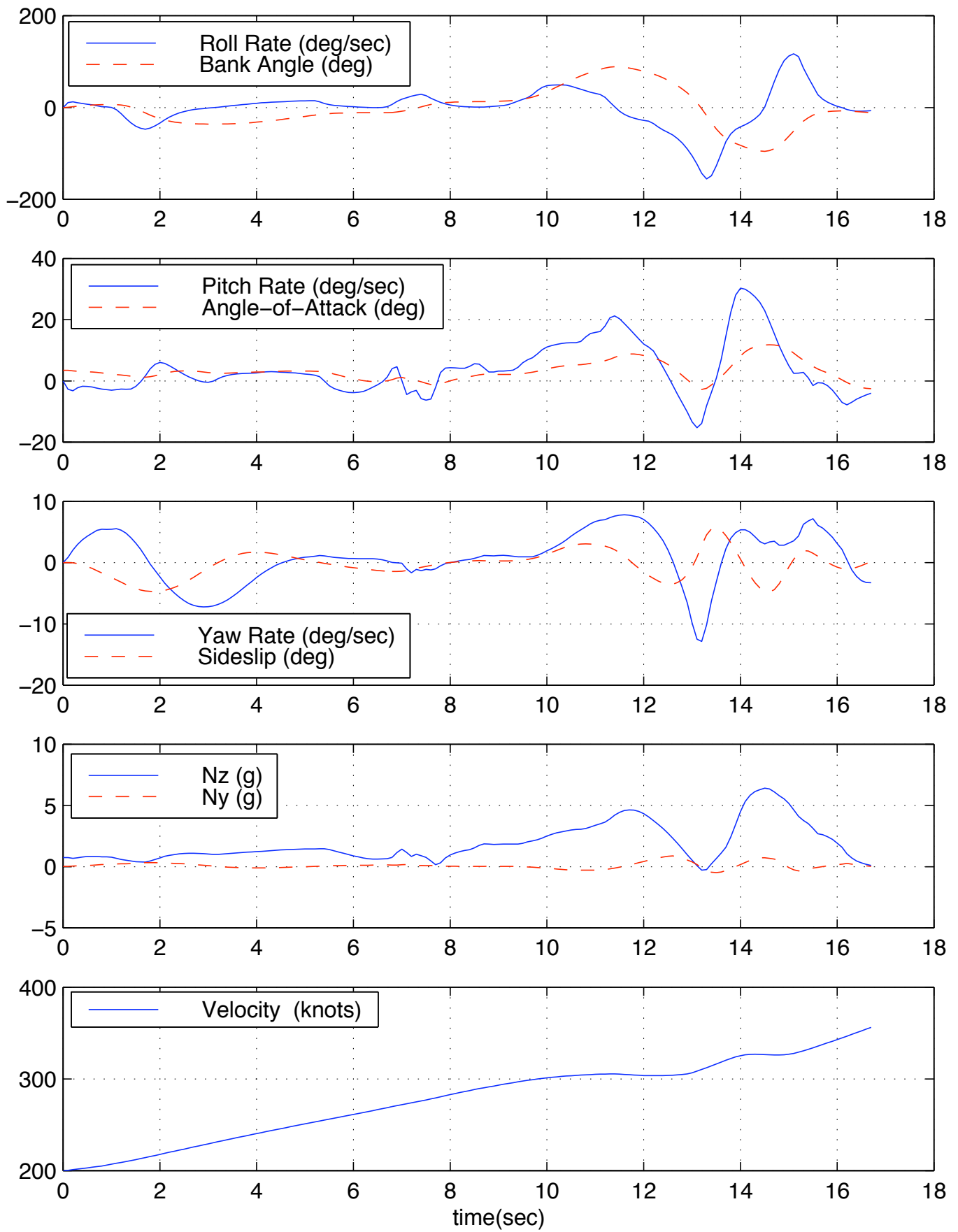

Figure 7.16: Aircraft states during terrain following task with double horizontal tail failure. 


\subsection{Viability of MRA for Control Reconfiguration}

Results presented in this chapter clearly indicate the capabilities of MRA to adapt to an identified control failure. In many cases, the control failure was transparent to the pilot. However, in several instances the control law's interpretation of the pilot's commands produced situations in which the output of the control law was a moment that fell outside the attainable moment subset. In these cases, the aircraft's actual response did not match the commanded moments. However, as previously noted, and reiterated for emphasis, no control allocation scheme can produce a combination of control effectors that achieves a moment that lies outside the AMS. With this statement in mind, one can go further to say that the control law should recognize the limitations of the AMS and not command moments that are unattainable.

One method of ensuring that the control law does not command moments outside the AMS is to utilize active stick logic that in real-time changes the position and rate limits on the control stick. By varying these parameters at every iteration, a one-to-one correspondence can be restored between available inceptor inputs and attainable effector position and rates. The changing of position and rate limits has the potential to eliminate the situation in which the pilot moves the stick past a point where the aircraft can not generate any further moment. This active stick logic has the possibility of offering the pilot feedback as to the performance capabilities of his aircraft, especially in the case of a control failure reconfiguration situation where the size of the resultant AMS has been reduced. Nonetheless, active stick logic is the subject of future research and is only mentioned here to present the notion of one way of informing the pilot of his loss of moment generating capability.

As an aside, the reader should note that no portion of MRA limits its use to tactical aircraft. The majority of the literature that investigates MRA implementation has focused on applications on fighter aircraft primarily due to research focus and funding issues. However, the utilization of MRA with its control failure reconfiguration capabilities on commercial aircraft or military transports is entirely feasible, given the proper control effectiveness data. In fact, MRA has the potential to save hundreds of lives if implemented on commercial aircraft. Consider USAir's flight 427 crash just outside Pittsburgh, PA on September 8, 1994. The aircraft involved in this crash was a Boeing 737-300. In a final report released in early 1999, the National Transportation Safety Board (NTSB) concluded that the primary cause of the crash was a rudder hard over. [24] A failure in the hydraulic system caused the rudder to 
become jammed at its blowndown limit. If a control failure identification and reconfiguration system had been installed on the aircraft 132 lives could have potentially been saved. If enough control power existed in the remaining control effectors, including ailerons, spoilers and flaps, these controls could have been used to counteract the tremendous yaw rate generated by the jammed rudder. Even if the aircraft performance was reduced after the failure, MRA would most likely have made the aircraft controllable and allowed the pilots to land safely.

The control failure reconfiguration capabilities of Moment Rate Allocation combined with a control failure identification algorithm can make commercial air travel safer in the future. As the number of new tactical aircraft projects dwindle, the utilization of MRA must expand its horizons by investigating new applications and markets and demonstrate its viability to make air travel around the world safer. It is the opinion of the author that it is the control reconfiguration capabilities of MRA that will ultimately earn its acceptance into the aircraft community as a viable method of control allocation. 


\section{Chapter 8}

\section{Performance Comparison}

\subsection{Euclidean Norm Comparison}

Comparing the Euclidean or 2-norm of control effector time histories for several allocation and restoring methods allows one to evaluate the allocators' ability to maintain control positions that are symmetric about the given zero deflection point. This data is useful because it presents results that analytically explain how far from the neutral position a given allocator keeps its controls during a maneuver. If the Euclidean norm of the control effectors is consistently high throughout a maneuver, it is indicative of controls being driven towards their stops. Controls at their stops in general will produce more drag on the aircraft as well as increase their potential for getting "wound-up". As mentioned in section 5.1, Leedy and Durham showed that control wind-up or Null Space Saturation did not have a crippling effect on MRA. However, it is not clear whether wind-up would produce performance degradation in the case of the F-15 ACTIVE's original control allocator.

The Euclidean norm of the controls is calculated using Matlab's "norm" command. The Euclidean norm, for a vector $\overrightarrow{\mathbf{x}}$, is given by equation 8.1. In words, the norm is the square root of the sum of the squares of each of the components. For this particular investigation, the components of the vector $\overrightarrow{\mathbf{x}}$ were the control effector positions for a given time step.

$$
\|\overrightarrow{\mathbf{x}}\|_{2}=\left[x_{1}^{2}+x_{2}^{2}+x_{3}^{2}+\cdots+x_{n}^{2}\right]^{1 / 2}
$$


Figure 8.1 shows time histories of the Euclidean norm of the control effector positions during a 4 inch lateral stick doublet at a flight condition of $400 \mathrm{knots}$ and 10,000 $\mathrm{ft}$ with 12 controls. The results for the original ACTIVE control allocator as well as MRA with several restoring methods are presented.
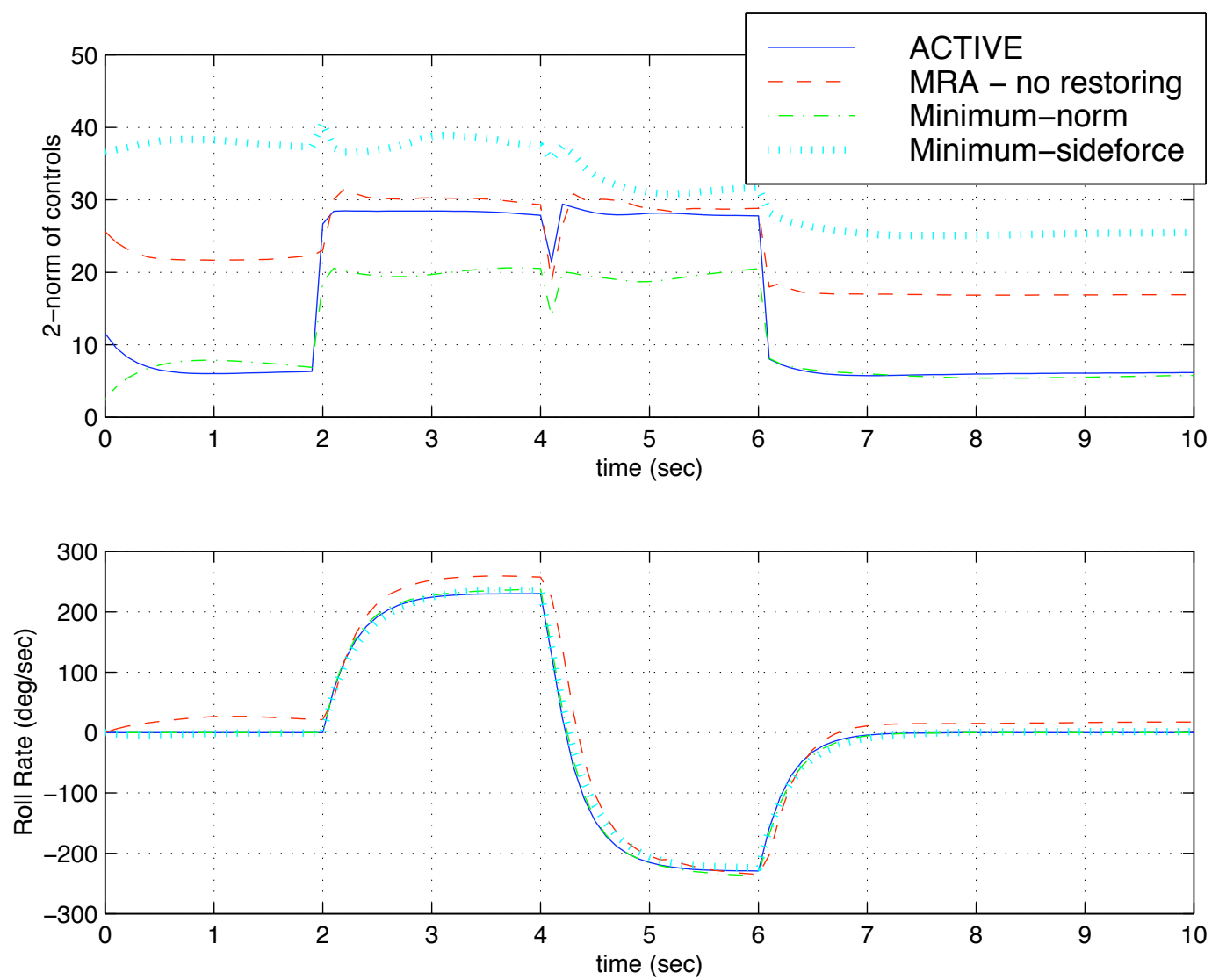

Figure 8.1: Euclidean norm of control effector positions as well as roll rate achieved during a 4 inch lateral stick doublet at 400 knots and 10,000 ft.

From Figure 8.1 it is clear that the minimum-norm restoring method, represented by the green dash-dot line, consistently produced the lowest value of the Euclidean norm throughout the maneuver. The ACTIVE mixer actually produces control effector configurations that are very similar to the minimum-norm restoring in terms of Euclidean norm. The ACTIVE's use of a symmetric and differential surface command in response to longitudinal and lateral stick inputs, respectively, creates a system that has a low Euclidean norm during periods of zero lateral stick input. However, during the lateral stick input, (ie. during the 2 to 6 second time period), the minimum-norm restoring outperforms the ACTIVE mixer with respect to 
minimizing the Euclidean norm. MRA without any restoring yields a Euclidean norm time history that is larger in magnitude than both the ACTIVE mixer and the minimum-norm restoring version of MRA. In addition, the minimum-sideforce restoring results are included in the figure to demonstrate that the minimization of sideforce does not necessarily produce control combinations with low Euclidean norms. In fact, the curve for minimum-sideforce restoring remains well above the other three curves throughout the entire maneuver. The second subplot of Figure 8.1 plots the time history of aircraft roll rate during the maneuver. This plot is included to remind the reader that the although the Euclidean norm of the control effectors varies between the different configurations investigated, the aircraft response is almost identical.

Evaluation of the Euclidean norm of control effector time histories for a real-time piloted task is performed to investigate the allocator's performance during a non-batch mode maneuver. Results from the High Yo-Yo of section 6.2 are used. Figure 8.2 shows a time history of the control effector norm for the solutions found by the ACTIVE mixer and MRA during the High Yo-Yo maneuver. The ACTIVE and MRA solutions were determined simultaneously during the simulation. For the results presented, the solution found by MRA was used to generate the aircraft response. It is clear from Figure 8.2 that MRA with minimum-norm restoring produced control combinations that yielded a lower Euclidean norm throughout the entire maneuver. Of interest in Figure 8.2 is the fact that the Euclidean norm curves for both allocators follow the same trends. The locations and magnitudes of peaks and valleys in the curves are very similar between the two allocators. Time histories of longitudinal and lateral stick inputs are included in the second subplot to demonstrate a correspondence between peaks in the Euclidean norm curve and lateral stick inputs. Larger lateral stick inputs demand larger differential control effector deflections and as a result produce increases in the Euclidean norm. The higher frequency lateral stick inputs produce perturbations from the lower frequency longitudinal stick "mean" value. 

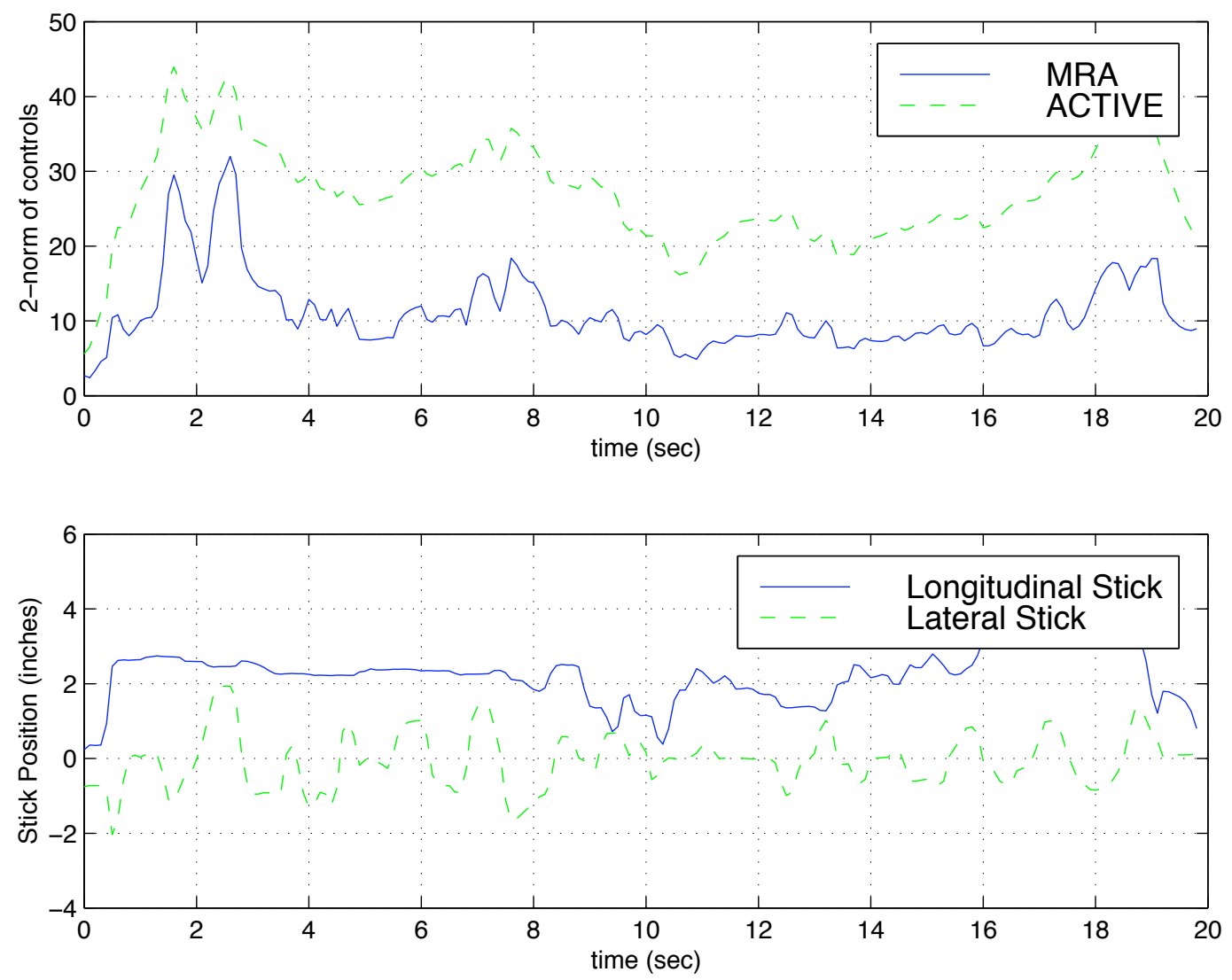

Figure 8.2: Euclidean norm of control effector positions for the ACTIVE mixer and MRA with minimum-norm restoring during the High Yo-Yo maneuver.

\subsection{Reduced Global Position Limits}

It has been stated that the Moment Rate Allocation method utilizing the Bisecting, EdgeSearching Algorithms yields the largest attainable moment subset of all control allocations schemes. However, to this point, no analytical data has been presented in this paper that demonstrates this point. It is the goal of this section to present data to the reader that clearly indicates an optimal performance of the MRA with BESA configuration. Due to its highly redundant suite of controls, very few maneuvers have been found that place the F-15 ACTIVE in a situation where its control effectors are position or rate saturated for extended periods of time. However, the reduction of the global control effector position limits by a factor of two produces a situation in which the control allocation schemes are regularly saturated. In fact, it will be shown that this reduction in control effector position limits renders the F-15 ACTIVE with its original mixer incapable of achieving the same level of 
aircraft performance as the same aircraft with MRA.

For simplicity, the rate limits on the control effectors were not reduced for this experiment. The primary justification for not reducing the rate limits is that the original F-15 ACTIVE control mixer did not account for rate limits and thus this reduction in rate limit would unfairly penalize MRA algorithms that do take rate saturation into account. One could argue that the original ACTIVE mixer was designed based on the availability of full control deflections, and that cutting these deflections in half is unfair. However, the purpose of this section is to demonstrate that MRA is flexible enough to utilize whatever control power is available, regardless of restrictions placed on control effector deflections. Table 8.1 shows the reduced control surface limits utilized in this experiment. Table 8.1 is identical to Table 2.1, but with control effector positions divided by a factor of two.

Table 8.1: Reduced control surface limits.

$\begin{array}{lcc}\text { Control Effector } & \text { Deflection (deg) } & \text { Rate Limit }(\mathrm{deg} / \mathrm{s}) \\ \text { DHT(L/R) } & -14.5 / 7.5 & \pm 46 \\ \text { DAIL(L/R) } & -10.0 / 10.0 & \pm 100 \\ \text { DCAN(L/R) } & -17.5 / 7.5 & \pm 23 \\ \text { DRUD(L/R) } & -15.0 / 15.0 & \pm 105 \\ \text { DTEF(L/R) } & 0.0 / 17.5 & \pm 18 \\ \text { DPV(L/R) } & -10.0 / 10.0 & \pm 80 \\ \text { DYV(L/R) } & -10.0 / 10.0 & \pm 80\end{array}$

The attainable moment subsets before and after the reduction in control position limits are given for a flight condition of $400 \mathrm{knots}$ and 10,000 ft in Figure 8.3. Note that these figures have the same shape as the attainable moment subsets discussed in chapters 4 and 7 .

The AMS shown in the lower portion of Figure 8.3 is identical to that above it, but the length of each edge of each facet has been reduced by a factor of two. In terms of reduction in moment generating capability, this change in control effector position limits has reduced the volume of the AMS to $3.3460 \mathrm{e}-3$ units $^{3}, 12.5 \%$ of its original volume. The corresponding changes in moment generating capability along each of the respective axes is listed in Table 8.2. 

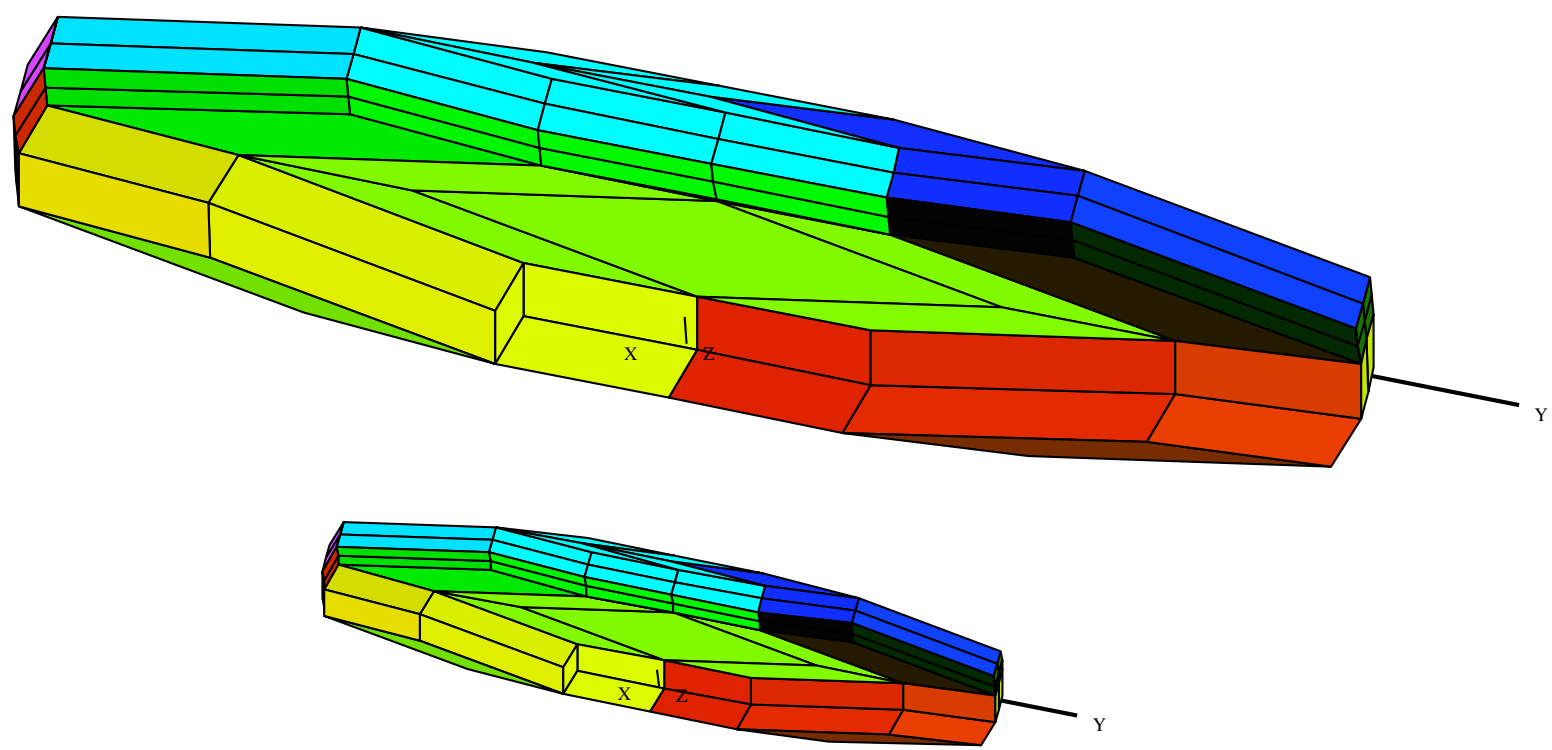

Figure 8.3: AMS before and after reduction in control effector position limits by a factor of two. F-15 ACTIVE at 400 knots and 10,000 ft.

$[\mathrm{htb}]$

Table 8.2: Minimum/maximum attainable moments for the F-15 ACTIVE with 12 controls in reduced control deflection configuration at $400 \mathrm{knots}$ and 10,000 ft.

\begin{tabular}{|l|c|c|c|c|}
\hline Moment & Min. Moment & \% Min. Moment* & Max. Moment & \% Min. Moment* \\
\hline \hline Roll & -0.03952 & 50.0 & 0.03951 & 50.0 \\
\hline Pitch & -0.25740 & 50.0 & 0.27188 & 50.0 \\
\hline Yaw & -0.05412 & 50.0 & 0.05413 & 50.0 \\
\hline
\end{tabular}

* Percentage of original moment without control failure.

The reduction in control effector position limits by a factor of two has produced a loss of $50.0 \%$ control moment generating capability along each of the three axes. the volume is $(0.5)^{3}$, or $12.5 \%$ of the original volume, as found above.

Now that the reduction in control capability has been pointed out, one can evaluate each of the control allocator's performances in this reduced configuration. The lateral stick doublet investigated previously will be evaluated because of its repeatability. 


\subsubsection{Lateral Stick Doublet: Reduced Controls Configuration}

The 4 inch lateral stick doublet previously discussed in section 7.3 is again evaluated. A graphical comparison of the roll rate generation capability of the original F-15 ACTIVE mixer and MRA control allocator is performed with the reduced controls configuration. Time histories of attained roll rate as well as control effector positions are plotted to show the effect of the reduction in control position travel on the allocation schemes. Figure 8.4 shows the roll rates attained by each of the allocators in response to 4, 3 and 2 inch lateral stick doublets. The non-reduced controls configuration is shown to indicate the performance of level of the aircraft in its original configuration. Minimum-norm restoring was utilized for the MRA case in this investigation.

The results depicted in Figure 8.4 clearly indicate that MRA outperforms the original F-15 ACTIVE mixer in the reduced control effector position limits configuration. In the case of the 4 inch doublet, although MRA does not achieve the same maximum roll rate as the original mixer in its non-reduced configuration, it is irrefutably superior to the original mixer's response in this case. The achieved roll rate is nearly double that which the original allocator achieved in this configuration. In fact, in the 3 inch lateral stick doublet, MRA with control reduction case was able to attain the same roll rate response as the original mixer without the reduction in control travel. The reduced control form of the original mixer fell close to 35 degrees/second shy of the attained maximum roll rate of 162 degrees/second for the 3 inch lateral stick doublet. All three allocators were able to achieve the 98 degrees/second commanded in the 2 inch doublet. This result is expected because with the F-15 ACTIVE's piecewise-linear stick gearing the 2 inch lateral stick doublet is within the capabilities of a $50 \%$ command in maximum roll rate.

Time histories of the control effector positions for the original F-15 ACTIVE mixer with MRA in response to a 3 inch lateral stick doublet are shown in figures 8.5 and 8.6. In Figure 8.5, the yellow hatching indicates periods of control effector position saturation for the original ACTIVE mixer. During the doublet, the ACTIVE's original mixer attempts to primarily use the horizontal tails and ailerons to generate the commanded roll rate. From the first subplot of Figure 8.5, it is clear that the ACTIVE mixer without the control reduction is commanding more horizontal tail deflection than is allowed in the reduced limits configuration. However, even though the control mixer knows about the reduced control effector limits, it does not change its allocation methodology and utilize other non-saturated 
control effectors to generate the commanded roll rate.

The original ACTIVE mixer's ailerons are also saturated during this maneuver as indicated in the second subplot of Figure 8.5. The ACTIVE mixer requires a couple more degrees of differential aileron deflection than is available in order to attain the commanded roll rate. However, the ACTIVE mixer fails to recognize the roll generating capabilities of the other available control surfaces, especially the canards. From the third subplot of Figure 8.5 one can clearly see that while MRA has taken advantage of the roll generation of a differential canard deflection, the ACTIVE mixer commands canard deflections that are in general symmetric. In fact, MRA has utilized a differential canard deflection that appears to generate a rolling moment in the opposite direction as that produced by the ailerons and horizontal tails. One could consider the ACTIVE mixer too structured and prioritized because it does not allow the flexibility to utilize all of its controls for moment generation about all axes. Since the canards are scheduled with angle-of-attack and used differentially primarily to regulate the angle-of-sideslip, their potential to generate the commanded roll rate that was not attained by the horizontal tails and ailerons is not recognized nor capitalized upon by the ACTIVE mixer.

Figure 8.6 shows time histories of the other six remaining controls. While none of the control effectors plotted in Figure 8.6 for the ACTIVE mixer with the reduced control limits become saturated during the maneuver, they are clearly not used to help generate the extra required roll rate. Very little yaw vectoring was used by the ACTIVE mixer while MRA took advantage of the yaw vectoring's capability to regulate the angle-of-sideslip.

The results of this lateral stick doublet are indicative of MRA's potential to maximize an aircraft's moment generating capability. In fact, this trial has shown that MRA experiences negligible degradation in aircraft performance even in the face of the reduction of control effector travel limits by a factor of two. On the contrary, the original F-15 ACTIVE mixer does not perform adequately under these conditions. The design of the F-15 ACTIVE's mixer does not optimize the potential of the control effectors to generate moments. MRA algorithms clearly outperform the original F-15 ACTIVE mixer in this lateral stick doublet investigation. 

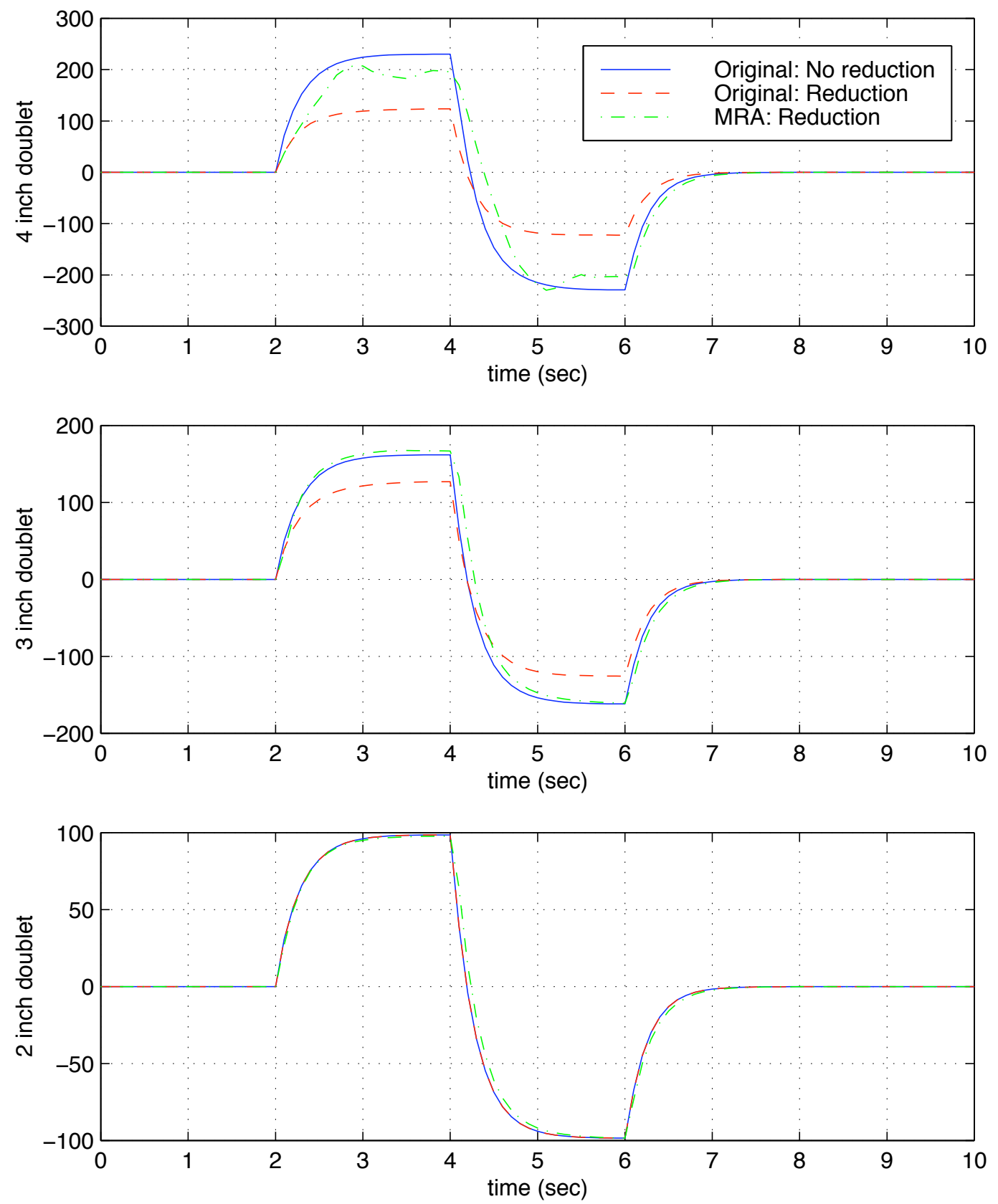

Figure 8.4: Roll rates achieved in reduced control effector position limits configuration for 4, 3 and 2 inch lateral stick doublets. Original mixer with and without control reduction as well as MRA mixer with reduced controls evaluated. 

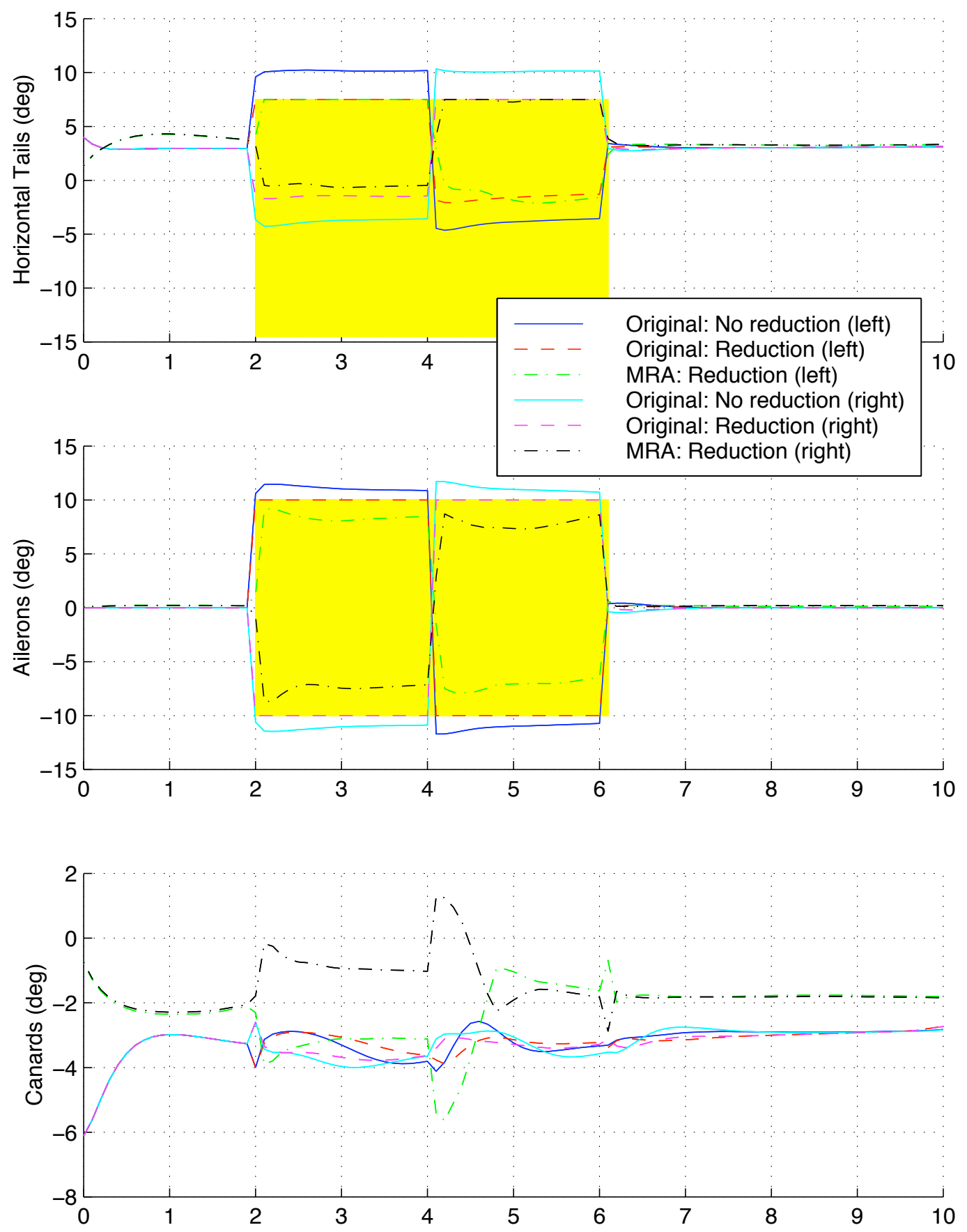

Figure 8.5: Horizontal tails, ailerons and canard responses to 3 inch lateral stick doublet with reduced control limits. Yellow hatching indicates the saturation of a control surface. 
Kevin R. Scalera
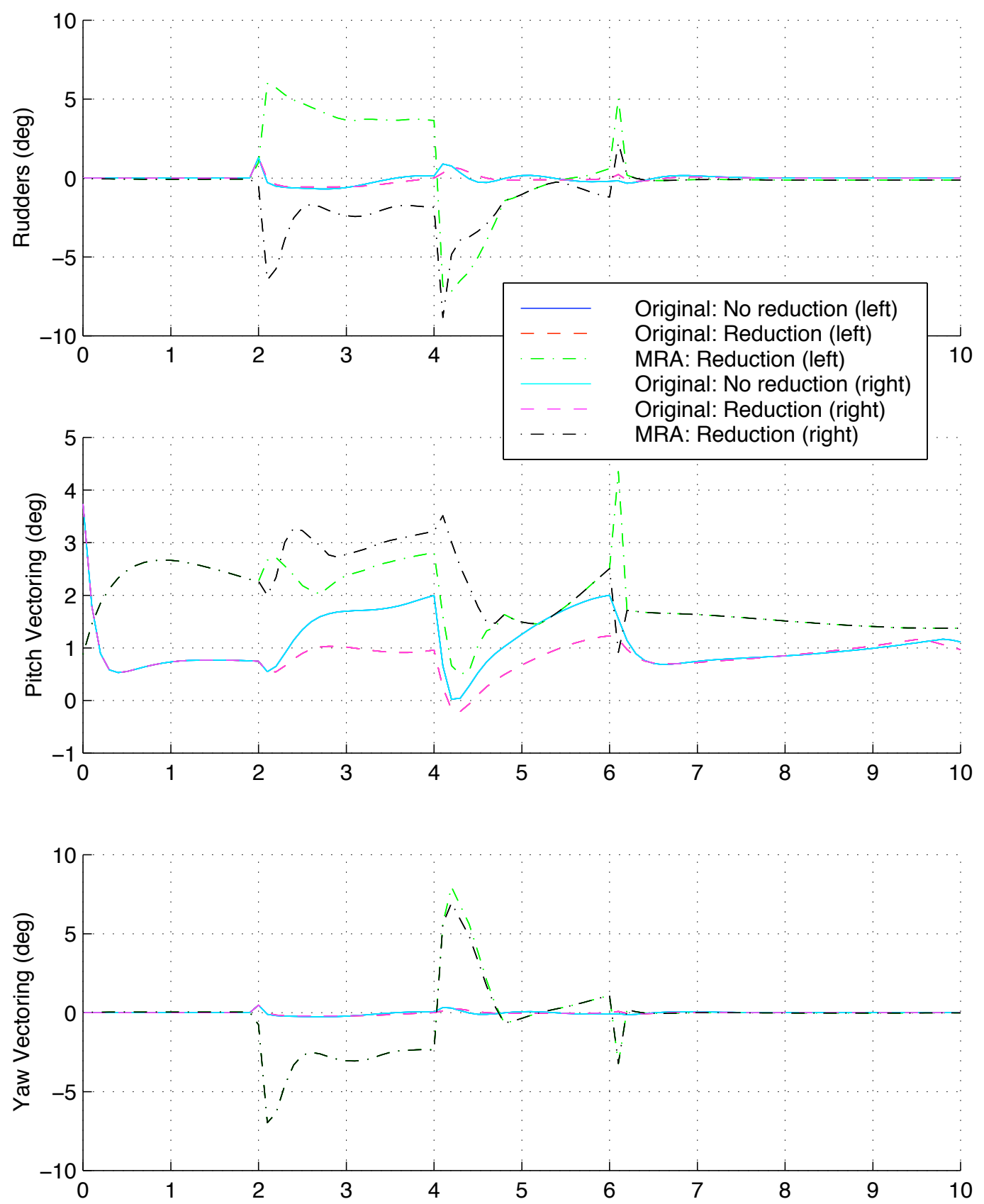

Figure 8.6: Rudder, pitch vectoring and yaw vectoring responses to 3 inch lateral stick doublet with reduced control limits. 


\section{Chapter 9}

\section{Summary and Conclusions}

A comparison of two control allocation methods was performed utilizing the F-15 ACTIVE research vehicle. Moment Rate Allocation utilizing the new Bisecting, Edge-Searching Algorithm (BESA) was implemented on the F-15 ACTIVE replacing the existing control allocator. The high-fidelity control law of the F-15 ACTIVE that accounted for aircraft handling qualities specifications was maintained. Real-time piloted simulations were completed to evaluate the performance of the allocators. Simulation results all indicated that the utilization of MRA with the BESA on a tactical aircraft with a highly redundant control suite is viable.

The framework of the new edge-searching algorithm was presented. A step-by-step algorithm was outlined for determining the control allocation solution. A graphical example was used to illustrate the procedure and offer assistance in the understanding of the algorithm. Although no code was presented that demonstrated the implementation of the new algorithm, the reader was presented with sufficient background to comprehend the concepts upon which the algorithm was built.

The timing concerns regarding MRA were alleviated with the implementation of the new edge-searching method. The number of floating point operations required for the new edgesearching algorithm was shown to increase in a linear manner. This linear increase in computational complexity as a function of number of control effectors is a significant computational savings in comparison to the quadratic relationship that existed utilizing the original bruteforce facet-searching algorithms. The edge-searching algorithm was designed with an upper limit on number of bisections per iteration. When this limit was attained and a solution had still not been found, an estimate of the solution was made. Although the number of required 
estimates increased with the number of control effectors, the percentage error associated with these estimations declined rapidly. The solutions for the edge-searching algorithm were thus deemed near optimal. This work therefore demonstrated the real-time implementation of a near optimal edge-searching algorithm and its potential real-time application to an actual tactical aircraft.

The idea of restoring methods was presented. Restoring methods tackle the goal of driving control effectors towards a desired configuration with the control power that remains after the primary objective is satisfied. Minimum-norm restoring, previously implemented with MRA in real-time simulations was once again investigated. In addition, minimum-sideforce restoring was presented. Minimum-sideforce restoring was shown to significantly reduce the total sideforce on the aircraft during maneuvers. This minimization of sideforce offers the advantage of eliminating the highly disconcerting and nauseating feeling that pilots undergo when the aircraft accelerates sideways.

In addition to the original method of restoring the controls utilizing a fixed step size scaling, the concept of variable step size restoring was introduced. It was shown that the utilization of a variable step size in the restoring offered a trade-off between restoring convergence time and reduction in control chatter. Although a technique was presented for variable step size minimum-sideforce restoring, an algorithm for variable step size minimum-norm restoring was not investigated. The determination of this variable minimum-norm step size algorithm as well as more sophisticated variable step size restoring algorithms that have foundations in optimization techniques remains the subject of future work. Variable step size restoring can be applied to any restoring method if a weighting for the determination of the step size can be calculated. A feasible application is that of minimum-drag variable step size restoring.

Representative maneuvers that offered realistic real-time evaluation of the allocator's performance and robustness were flown with a pilot-in-the-loop. The High Yo-Yo, a well known air combat maneuvering task, was investigated to evaluate the allocator's performance in an aggressive pilot tracking task. In addition, a terrain following task was flown in order to evaluate the allocator's performance in a low dynamic pressure flight condition. Desired and adequate performance levels were established for each of these tasks. Results from the High Yo-Yo indicated that the task could regularly be completed within the desired time and distance constraints. Although the terrain following task was found to be extremely difficult to complete, a success rate of approximately $25 \%$ was sufficient to produce the necessary data for analysis. 
Control failure reconfiguration in the event of an identified failure was investigated. Two feasible control failure combinations were evaluated. An aileron hard-over as well as the loss of both the left and right horizontal tails were implemented. The reduction in moment generating capability was presented for each of the failure cases. The failures were incorporated on the aircraft during the representative maneuvers. Evaluation of the High Yo-Yo with both the aileron hard-over and the double horizontal tail failure yielded results that indicated that the failures did not significantly degrade the aircraft's performance. The allocator was able to successfully utilize the remaining control effectors to maximize the moment generating capability of the aircraft in the control failure configuration. Results from the terrain following task were not as conclusive. The demands of the maneuvers made the completion of the task extremely difficult with the reduction in moment generating capability associated with the control failure. However, the completion of the task approximately $15 \%$ of the time with control failures indicated that a significant level of aircraft performance was maintained in the failed configuration. In addition, although data was not presented in this work, experimentation with powered approaches to an aircraft carrier, a similar flight condition to that of the terrain following task, revealed that the aircraft remained controllable enough to repeatedly perform successful landings with control failures.

A further investigation into each of the allocators capability to perform in a reduced control deflection configuration was performed. Control travels were reduced by a factor of two. A lateral stick doublet was simulated. The roll rate response of the aircraft with each of the control allocators was recorded. Results indicated that the F-15 ACTIVE's original control allocator lacked the sophistication to utilize the redundancy of the controls to overcome the reduction in their travel limits. The results from the MRA simulation demonstrated the capabilities of MRA to utilize the entire AMS to generate the desired moment. In fact, due to the high redundancy of the control effector suite, one can state that there was negligible degradation in aircraft performance with MRA even with the control effector travel limits reduced by a factor of two. This experiment illustrated a clear advantage of utilizing MRA with its guarantee to maximize the moment generating capabilities of the control effector suite.

The results from the control failure reconfiguration and the reduced control deflection configuration investigations indicate the potential of MRA to maintain satisfactory aircraft performance by reconfiguring the controls in the event of an identified failure. In fact, in the case of the High Yo-Yo, the pilot was able to complete the mission with little noticeable 
degradation in aircraft performance. It is valid to hypothesize that control reconfiguration is easy with the F-15 ACTIVE due to its suite of highly redundant control effectors. However, one must consider that the allocator that currently exists on the aircraft did not encompass the facilities to perform control reconfiguration. Furthermore, it can be surmised that the majority of existing aircraft control law - control allocator combinations do not incorporate control reconfiguration algorithms. It is this control reconfiguration capability, combined with the guarantee that the entire AMS is utilized in the control solution, that sets MRA apart from the rest of the currently existing control allocation methods. It is this combination that will gain MRA acceptance into the aircraft community as a robust and viable control allocation method for utilization on any aircraft with a redundant suite of control effectors.

The results presented in this paper have demonstrated the capabilities of utilizing MRA in real-time piloted simulations. The next logical step is implementation in an actual aircraft for flight test evaluation. However, before MRA can graduate to this next level, several questions must be addressed. The phenomenon referred to control law - control allocator interaction must be investigated. The original assumption that these two items were completely independent of one another is no longer valid. The necessity of transforming the F-15 ACTIVE's outputs to a different coordinate system, and the variation in aircraft response with different transformation matrices, clearly indicated that outputs of a control law could be tailored to fit the type of inputs that a control allocator desires. A better understanding of this interaction must be gained through thorough evaluation of combinations of control law structures and MRA. However, it should be noted that although an interaction did exist between the control law and the control allocator, this interaction did not cause a degradation in aircraft performance.

All control reconfiguration performed in this research assumed that the control failure was identified. Therefore, the development of the techniques and algorithms required to identify the failure will be the subject of future work. Nonetheless, it may not be necessary to reinvent the wheel in this case. Current research in this area has produced viable control failure identification algorithms. [21] Unfortunately, not all of these existing algorithms are presently suited for real-time implementation. [25] However, the combination of the current MRA reconfiguration algorithms and one of the more mature control failure identification algorithms promises the realization of a highly robust adaptive aircraft control system. 


\section{Bibliography}

[1] Durham, Wayne C. and Leedy, Jeffrey Q., "Real-Time Evaluation of Control Allocation with Rate Limiting" AIAA-98-4460,1998.

[2] Bolling, J. Implementation of Constrained Control Allocation Techniques Using an Aerodynamic Model of an F-15 Aircraft, Master's Thesis, Virginia Polytechnic Institute and State University, Blacksburg, VA, 1997.

[3] Durham, W. C., "Computationally Efficient Control Allocation" AIAA-99-4214,1999.

[4] Durham, W. C., "Attainable Moments for the Constrained Control Allocation Problem" Journal of Guidance, Control, and Dynamics, Volume 17, Number 6, 1994, pp. 13711373.

[5] Durham, W. and Bordignon, K. "Multiple Control Effector Rate Limiting" Journal of Guidance, Control, and Dynamics, Volume 19, Number 1, 1996, pp. 30-37.

[6] Smolka, Jim, et. al., "F-15 ACTIVE Flight Research Program" As published in the Society of Experimental Test Pilots $40^{\text {th }}$ Symposium Proceedings.

[7] Buckley, James E., "Versatile Flight Control System Functional Description ACTIVE Application" Unpublished 1995 by McDonnell Douglas Corporation.

[8] Chalk, C. R., Neal, T. P., Harris, T. M., Pritchard, F. E. and Woodcock, R. J., Background Information and User Guide [BIUG] for Mil-F-8785B (ASG), Military Specification - Flying Qualities of Piloted Airplanes, AFFDL-TR-69-72, 1969, pp. 13.

[9] Reiner, Jacob, Gary J. Balas and William L. Garrard, "Robust Dynamic Inversion for Control of Highly Maneuverable Aircraft" Journal of Guidance, Control, and Dynamics, Volume 18, Number 1, 1995, pp. 18-24. 
[10] Brinker, Joseph S., and Kevin A. Wise, "Stability and Flying Qualities Robustness of a Dynamic Inversion Aircraft Control Law" Journal of Guidance, Control, and Dynamics, Volume 19, Number 6, 1996, pp. 1270-1277.

[11] Durham, W., "AOE5214: Aircraft Dynamics and Control" Class notes from fall 1998, Aerospace and Ocean Engineering Department, Virginia Polytechnic Institute and State University, 1998.

[12] Nichols, James H., Magyar, Thomas J. and Schug, Eric C., "The Platform-Independent Aircraft Simulation Environment at Manned Flight Simulator" AIAA-98-4179,1998.

[13] Scalera, Kevin R., and Durham, W., "Modification of a Surplus Navy 2F122A A-6E OFT for Flight Dynamics Research and Instruction" AIAA-98-4180,1998.

[14] Grantham, William D. and Robert H. Williams, "Comparison of In-Flight and GroundBased Simulator Derived Flying Qualities and Pilot Performance for Approach and Landing Tasks" AIAA-87-2290,1998.

[15] Durham, W., "Constrained Control Allocation" Journal of Guidance, Control, and Dynamics, Volume 16, Number 4, 1993, pp. 717-725.

[16] Durham, W., "Constrained Control Allocation: Three Moment Problem" Journal of Guidance, Control, and Dynamics, Volume 17, Number 2, 1994, pp. 330-336.

[17] Durham, W., "Control Stick Logic in High Angle-of-Attack Maneuvering" Journal of Guidance, Control, and Dynamics, Volume 18, Number 5, 1995.

[18] Durham, W., Bolling, J., and Bordignon, K. "Minimum Drag Control Allocation" Journal of Guidance, Control, and Dynamics, Volume 20, Number 1, 1997, pp. 190-193.

[19] Bordignon, K. A., Constrained Control Allocation for Systems with Redundant Control Effectors, Ph.D. Dissertation, Virginia Polytechnic Institute and State University, Blacksburg, VA, 1996.

[20] Shaw, Robert L., Fighter Combat: Tactics and Maneuvering , Naval Institute Press, Annapolis, Maryland, 1985, pp. 71-73.

[21] Enns, Dale, Michael Elgersma and Petros Voulgaris, Parameter Identification for Systems with Redundant Actuators, Honeywell Technology Center, MS MN65-2810, January 1998, Draft. 
[22] Ziegler, G. M., Lectures on Polytopes, vol. 152, First (Revised) ed. Heidleberg: SpringerVerlag, New York Berlin, 1995, pp. 370.

[23] Hodgkinson, John, Aircraft Handling Qualities, American Institute of Aeronautics and Astronautics, Inc., Reston, Virginia, 1999, pp.4-19.

[24] National Transportaion Safety Board, "Public Meeting of March 23-24, 1999: Abstract of Final Report - USAir flight 427 Crash in Aliquippa, PA, September 8,1994" NTSB March 23-24, 1999

[25] Chandler, P. R., M. Pachter and M. Mears, "System Identification for Adaptive and Reconfigurable Control ", Journal of Guidance, Control, and Dynamics, Volume 18, Number 3, 1995, pp. 516-524.

[26] Duke, Lee, "NASA Facts: F-15 ACTIVE Advanced Control Technology for Integrated Vehicles", NASA Dryden Flight Research Center, http://www.dfrc.nasa.gov/ PAO/PAIS/HTML/FS-048-DFRC.html, FS-1998-060048-DFRC, June 1998.

[27] Stevens, Brian L. and Frank L. Lewis, Aircraft Control and Simulations, John Wiley \& Sons, Inc., New York, 1992, pp. 593-607. 


\section{Appendix A}

\section{F-15 ACTIVE Specifications}

This appendix contains dimensions and specifications for the F-15 ACTIVE as well as a summary of the ACTIVE project and some of its testbed experiments. These pages were taken from the NASA Dryden Flight Research Center's web page. [26]

$$
\text { http://www.dfrc.nasa.gov/PAO/PAIS/HTML/FS-048-DFRC.html. }
$$

All information is valid as of June 1998. For updated information, it is suggested that the reader personally visit the above listed web page. In addition, the reader is referred to the ACTIVE home page.

$$
\text { http://www.dfrc.nasa.gov/Projects/active/index.html }
$$

The material in this section is presented as fact and not interpreted or modified at all by the author. 


\section{NASAFacts \\ National Aeronautics and Space Administration \\ Dryden Flight Research Center \\ P.0. Box 273 \\ Edwards, California 92523 \\ AC 205 258-3449 \\ FAX 805 358-3556 \\ F-15 ACTIVE \\ Advanced Control Technology for Integrated Vehicles}

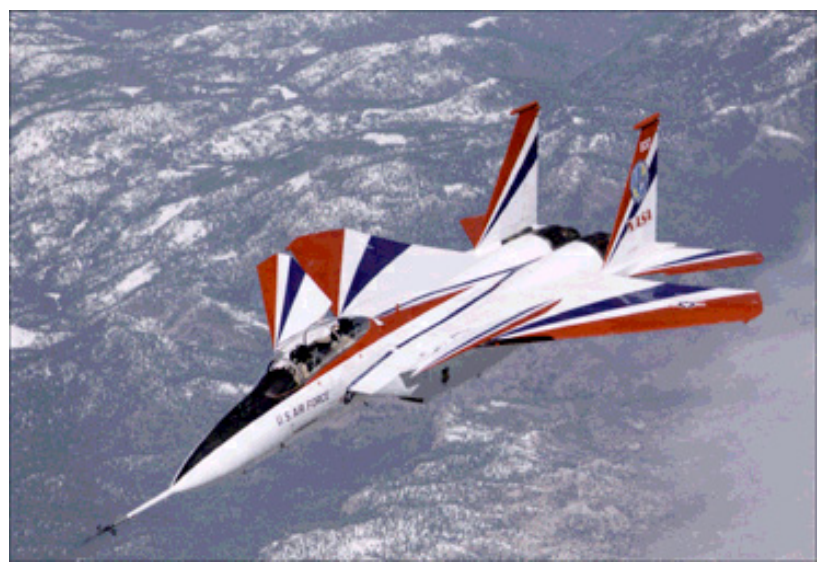

F-15B ACTIVE in flight, NASA photo EC96 4348513

\section{Project Summary}

The Advanced Control Technology for Integrated Vehiclesor "ACTIVE"program at NASA's Dryden Flight Research Center is a multi-year flight research effort to enhance the performance and maneuverability of future civil and military aircraft. For this program, advanced flight control systems and thrust vectoring of engine exhaust have been integrated into a highly-modified F-15 research aircraft. The program is a collaborative effort by NASA, the Air Force Research Laboratory, Pratt \& Whitney, and Boeing (formerly McDonnell Douglas) Phantom Works. The ACTIVE program supports the Revolutionary Technology Leaps pillar of NASA's Aeronautics and Space

Transportation Technology Enterprise by revolutionizing the way in which aircraft are designed and built by providing the design tools to increase design confidence and cut design time for next-generation aircraft in half.

\section{Aircraft Description \& Modifications}


The F-15 ACTIVE research aircraft, the first two-seat F-15 built by McDonnell Douglas, was used initially for developmental testing and evaluation. In the mid 1980's, the aircraft was extensively modified for the Air Force's Short Takeoff and Landing Maneuvering Technology Demonstrator (S/MTD) program. Those modifications included equipping the aircraft with a digital fly-by-wire control system, canards (modified F-18 horizontal stabilators) ahead of the wings and two-dimensional thrust-vectoring, thrust-reversing nozzles which could redirect engine exhaust either up or down, giving the aircraft greater pitch control and aerodynamic braking capability.

After being loaned to NASA for the ACTIVE program, the twin-engine F-15 was equipped with a powerful research computer, higher-thrust versions of the Pratt \& Whitney F-100 engine and newly developed axisymmetric thrust-vectoring engine exhaust nozzles that are capable of redirecting the engine exhaust in any direction, not just in the pitch (up and down) axis or direction.

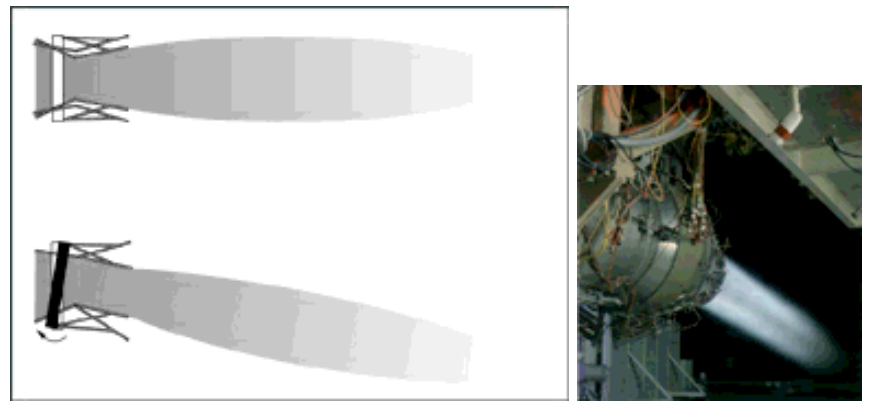

Thrust vectoring (left) Pratt \& Whitney's Yaw Balanced Beam Nozzle (right)

The new nozzles can deflector vectorengine thrust Up to $20^{\circ}$ off center line, giving the aircraft thrust control in pitch (up and down) and yaw (left and right), or any combination of the two axes. This deflected (vectored) thrust can be used to reduce drag and increase fuel economy or range as compared with conventional aerodynamic controls. The nozzles are a production design that could be incorporated into current or future aircraft.

In addition, an integrated system to control its aerodynamic control surfaces and its engines was installed in the ACTIVE F-15 along with cockpit controls and electronics from the F-15E.

\section{Project Status}

Several flight research milestones have been recorded in the ACTIVE program to date. The first supersonic yaw-vectoring flight was flown in early 1996, and pitch and yaw thrust vectoring at speeds up to Mach 2twice the speed of sound was evaluated during several flights late in the year. On subsequent flights, Dryden research pilots flew the F-15 ACTIVE at angles of attack up to $30^{\circ}$ while employing yaw vectoring."Angle of attack" describes the relationship between the aircraft's body and wings to its actual flight path. 


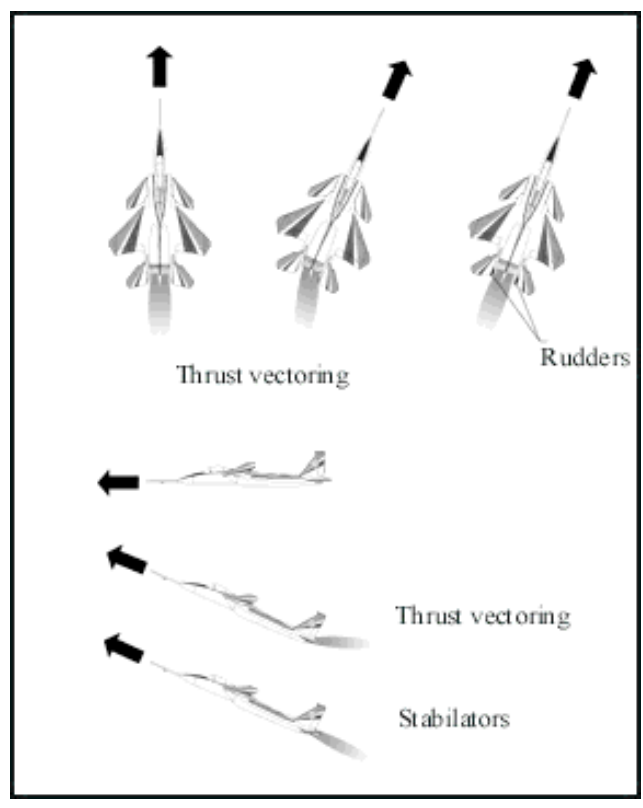

An adaptive performance software program was developed and successfully tested. The performance-optimization program installed in the aircraft's flight control computer automatically determines the optimal setting or trim for the thrust-vectoring nozzles and aerodynamic controls to minimize aircraft drag. On the last flight of 1996, the F-15 ACTIVE demonstrated the software's effectiveness by gaining a speed increase of Mach 0.1 with no increase in engine power while in level flight at 30,000 ft altitude and a speed of approximately Mach 1.3.

The F-15 ACTIVE has continued to expand the limits of its thrust-vectoring capabilities during 1997 and 1998, including an experiment which combined thrust vectoring with its regular aerodynamic controls to improve the performance of the F-15E tactical fighter on ground attack missions.

\section{Testbed Experiments}

The F-15 ACTIVE's unique propulsion control systems and flight test instrumentation have allowed it to be used as a testbed for several research experiments unrelated to the ACTIVE program. Each experiment contributed to goals which will benefit Global Civil Aviation, another one of the three pillars of NASA's Aeronautics and Space Transportation Technology Enterprise.

HIGH STABILITY ENGINE CONTROL (HISTEC) This experiment, developed and managed by NASA's Lewis Research Center, evaluated a computerized system that can sense and respond to high levels of engine inlet airflow turbulence to prevent sudden in-flight engine compressor stalls and potential engine failures. The system used a high-speed processor to process the airflow data coming from sensors on the left engine, and it in turn directed the aircraft's engine control computer to automatically command engine trim changes to accommodate for changing turbulence levels. The system can enhance engine stability when the inlet airflow is turbulent, and increase engine performance when the airflow is stable or smooth. Approximately one dozen flights were flown in the summer of 1997 to validate the HISTEC concept. The project contributed to the Affordable Air Travel goal of significantly reducing the cost of air travel, and the Safety goal to reduce the aircraft accident rate. 
HIGH-SPEED RESEARCH ACOUSTICS The unique ability of the thrust-vectoring nozzles to change the "area ratio"the difference in the geometric areabetween the nozzles' throat and exit led to the F-15 ACTIVE being used for research in the fall of 1997 on how to reduce perceived engine noise. Conducted on behalf of Langley Research Center's High-Speed Research program, this flight experiment focused on validating noise prediction data that could be applied to reducing noise generated during takeoffs and landings of the High Speed Civil Transport, the proposed second-generation American supersonic jetliner. By fully expanding the nozzles' exit area, noise generated by the hot jet exhaust entering the surrounding cooler air is reduced. The acoustics research involved flying the F-15 ACTIVE in precise patterns over an array of 30 microphones spread out over more than a mile along the northeast side of Rogers Dry Lake. The project contributed to the Environmental Compatibility goal of significantly reducing the perceived noise levels of future aircraft.

INTELLIGENT FLIGHT CONTROLThis experiment, planned for flight testing in late 1998-early 1999 , is intended to assist development of advanced "neural network" flight control computer technology that would allow aircraft control systems to adapt to unforeseen changes in aircraft operating conditions, such as sudden equipment failures or battle damage, by directing the aircraft's remaining functional control systems to compensate for the failure or damage. Successful development and validation of the Intelligent Flight Control concept will contribute to NASA's Safety goal by allowing safe return of aircraft that otherwise might be uncontrollable after sustaining damage or major system failures.

\section{Technology Commercialization}

The overall goal of the ACTIVE program is to help develop technology for the next generation of high-performance civil and military aircraft, as well as significantly cut the time spent in design by reducing complexity. Applying new integrated flight/propulsion control technology can lead to development of revolutionary new designs which will be lighter, less complex, less costly, and with greatly improved performance as conventional aerodynamic controls and their systems are reduced or eliminated.

\section{Aircraft Statistics}

The F-15 is a versatile aircraft, employed by the U.S. Air Force as its premier air-superiority fighter/interceptor aircraft as well as its long-range all-weather strike fighter. It is an ideal aircraft for the ACTIVE research role. 


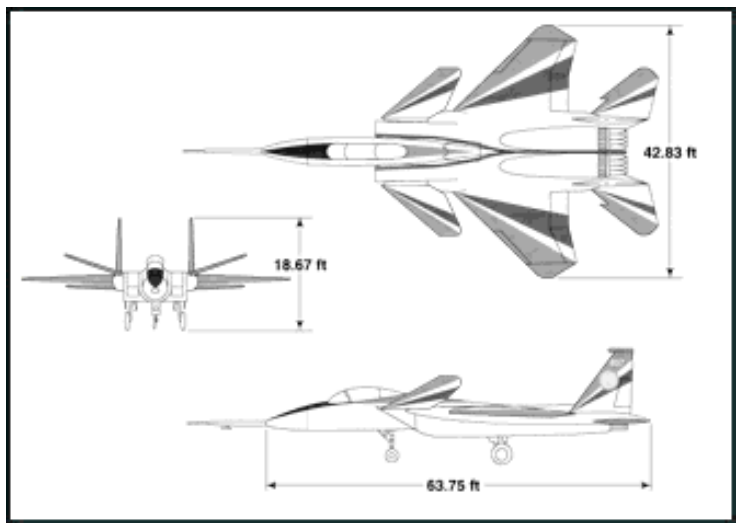

Three view of F-15B ACTIVE.

Designation: F-15B, originally TF-15A Manufacturer: McDonnell Douglas, 1972

Owner: United States Air Force USAF Registration: 71-290

NASA registration: (tail number) 837 NASA role: Integrated controls/propulsion research

Maximum altitude: 60,000 ft Max. speed: Mach 2.0

Engines: Two Pratt \& Whitney F100-PW-229 Max. thrust: 29,000 lb in full afterburner each

Weight: 47,000 lb takeoff; 35,000 lbs empty Fuel capacity: 11,520 lb (approx. 1,700 gal)

Wingspan: $42.8 \mathrm{ft}$ Length: $63.7 \mathrm{ft}$, excluding flight test nose boom

Horizontal tail span: $28.2 \mathrm{ft}$ Canard span: $25.6 \mathrm{ft}$

NASA Dryden Flight Research Center

Public Affairs Office

Edwards, CA 93523

(661)258-3449

FAX (661)258-3566

Document number: FS-1998-06-048-DFRC

Responsible NASA Official: Lee Duke

Web Page Curator: Linda Peters

Last modified: June 1998 


\section{Appendix B}

\section{Control Effectiveness Extraction Code}

\section{B.1 Subroutine Bgetter.c}

\section{B.1.1 Description}

Subroutine BGETTER produces an executable code that generates formatted table lookups for control effectiveness. The calculated partial derivatives are returned in the standard CASTLE FTP table look-up format. Breakpoints are given for angle-of-attack, Mach number, and altitude. Additionally, for the thrust-vectoring controls, breakpoints are added for the power lever angle (PLA). The ability to attain sideforce effectiveness is also built into the program. Two files are produced for each axis evaluated. One contains data for a flaps-up (up-and-away) configuration and the other contains data for a flaps-down (powered approach) configuration. The files for the pitching moment are named "dm.dat" and "dm pa.dat", respectively. All other file names follow accordingly.

For each data point calculated, a call is made to subroutine JACOB. Subroutine JACOB, described in section B.2, determines the partial derivative of the given moment with respect to the given control.(ie. the B matrix) Although JACOB has the capability to calculate the partial derivative of a vector of moments with respect to a vector of controls, only one partial derivative is calculated at each iteration in order to simplify the formating of the table look-up output files. 


\section{B.1.2 Code}

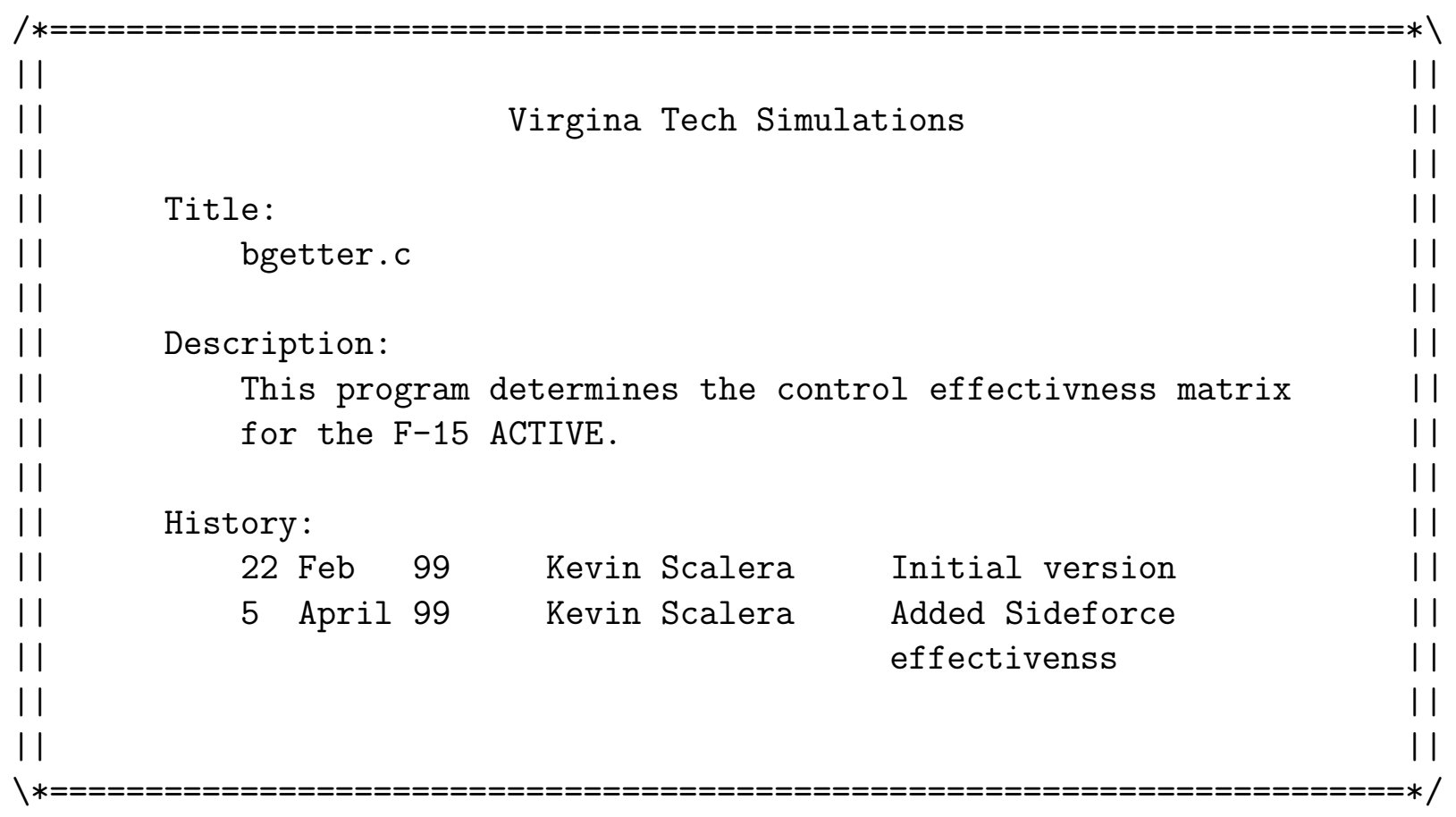

/* Standard include files */

\#include <stdio.h>

\#include <stdlib.h>

\#include <string.h>

\#define AIRFRAME_GENERATE

/* CASTLE include files */

\#include "airframe_exec.h"

\#include "xatmos.h"

\#include "xf15active.h"

\#include "shell1.h"

\#include "shell2.h"

/* Jacobian subroutine declaration */

extern int jacob_(long*,long*,float*,long*,long*);

void main( ) 


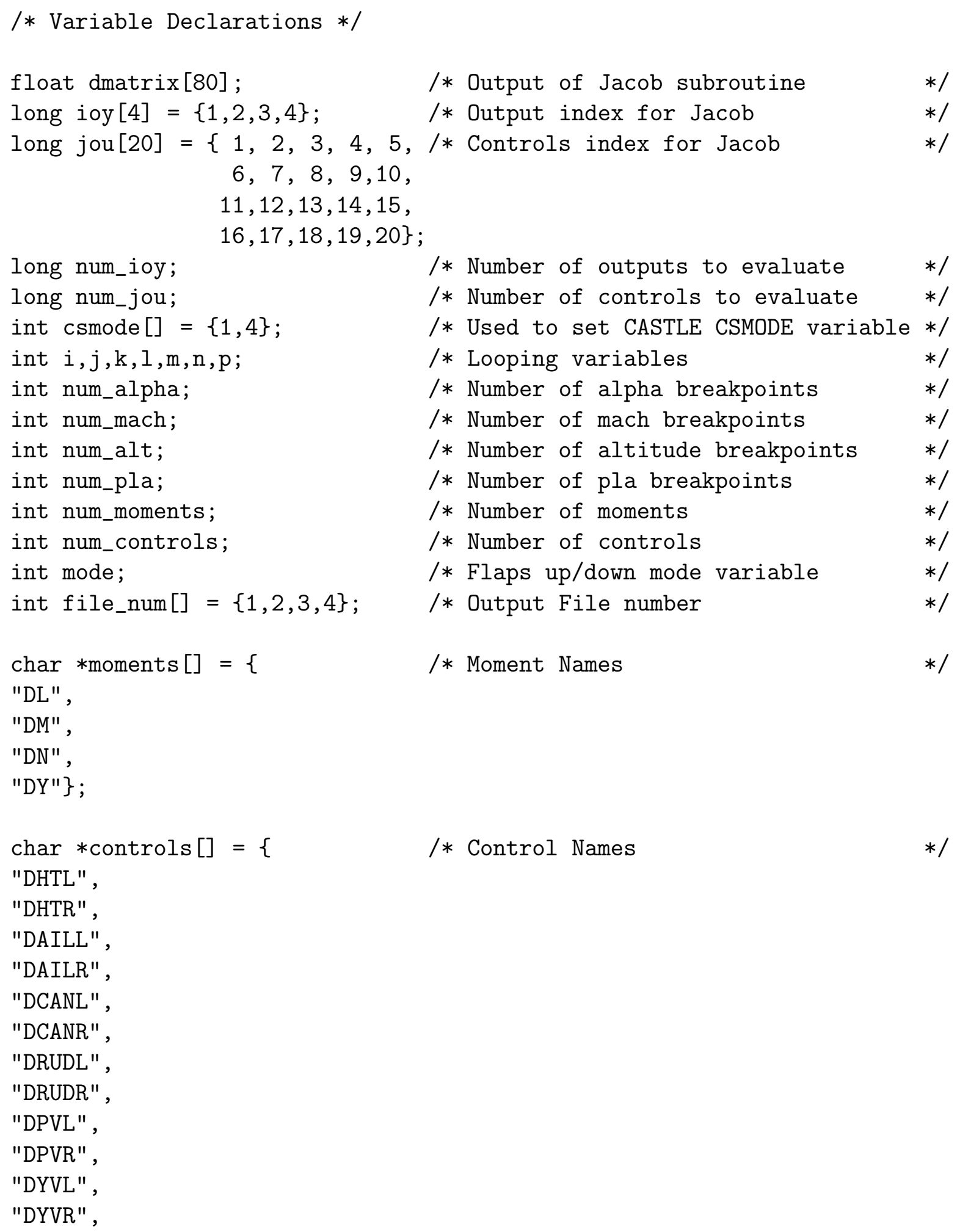




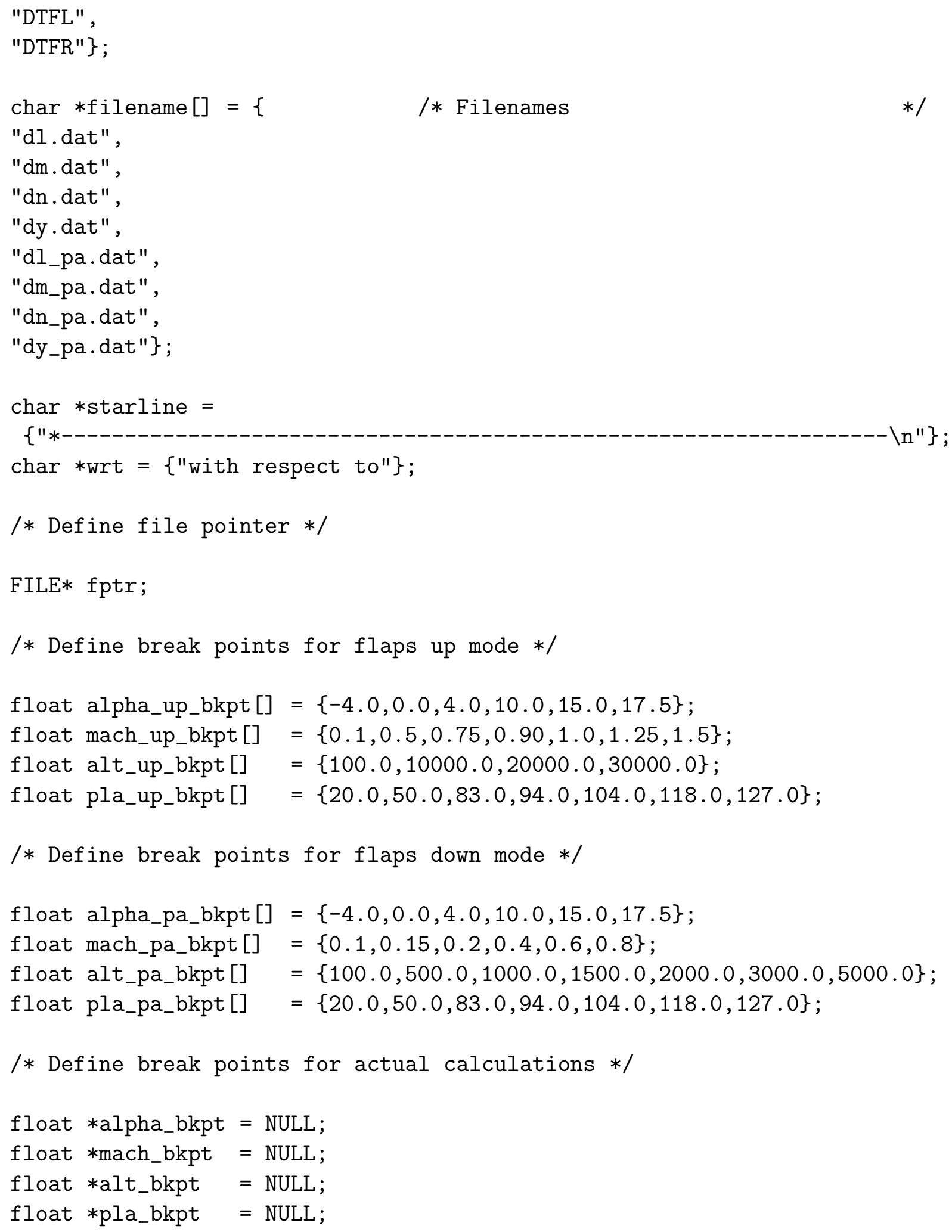




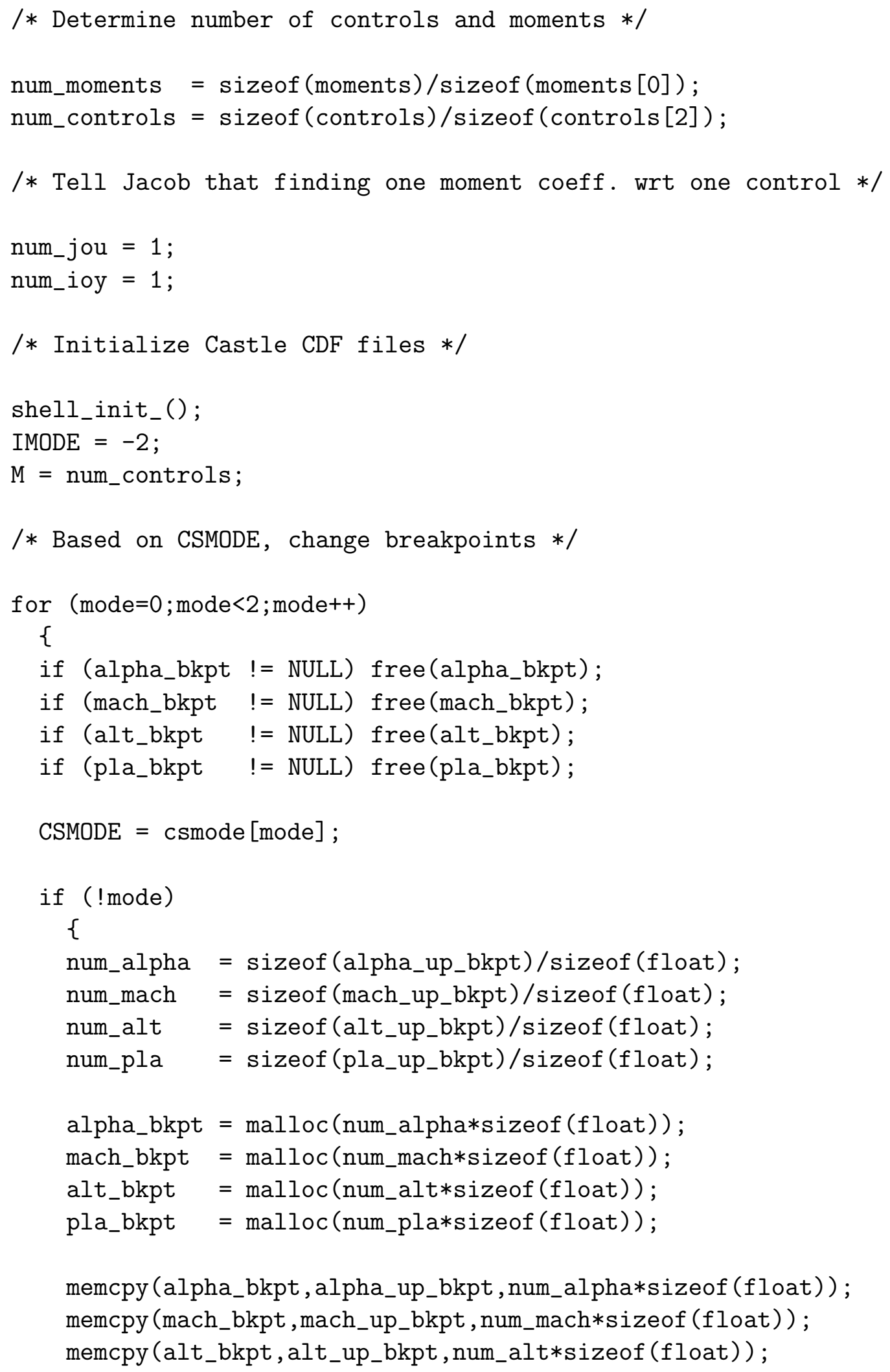




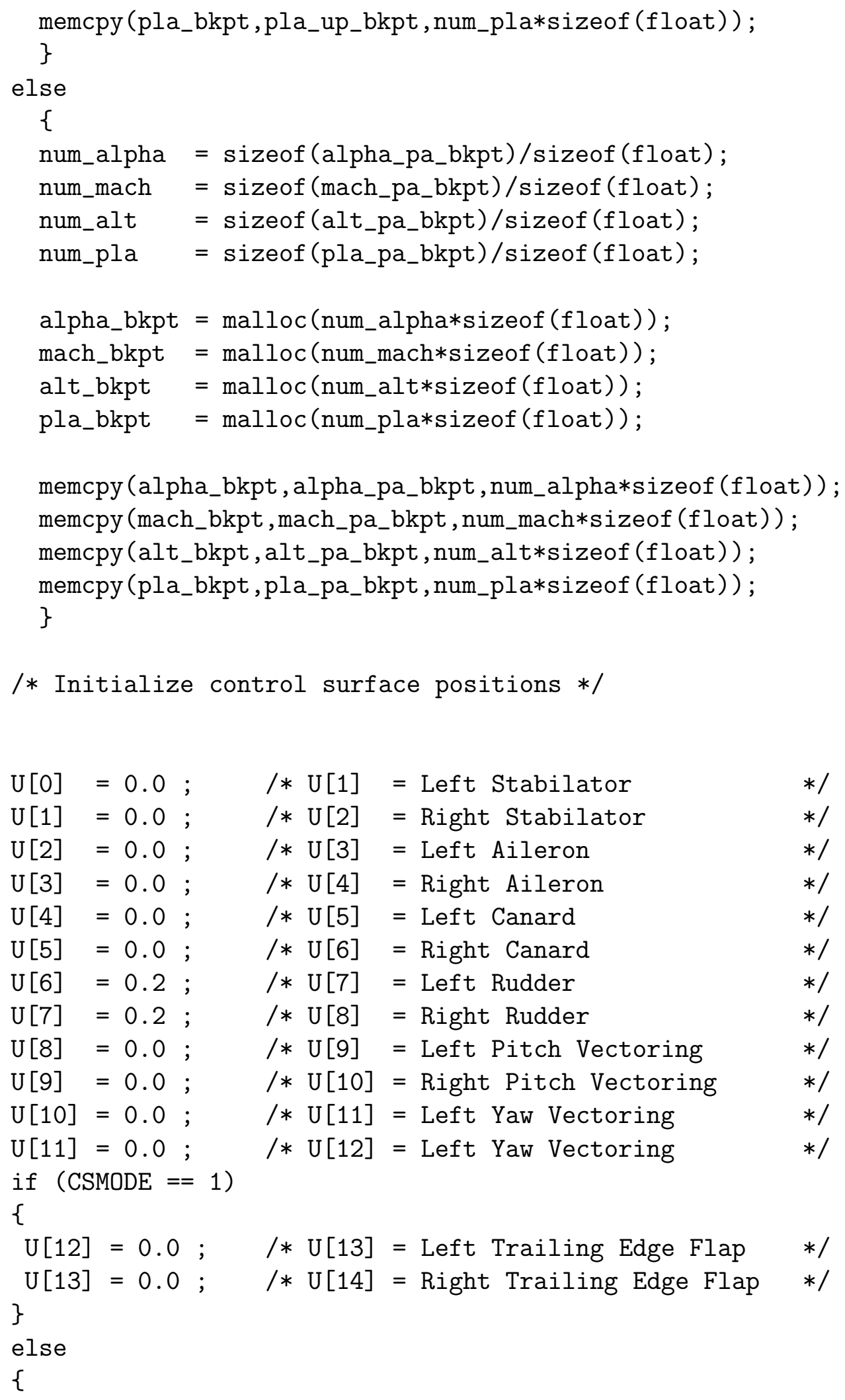




$\begin{array}{llll}\mathrm{U}[12]=20.0 ; & / * \mathrm{U}[13] & =\text { Left Trailing Edge Flap } & * / \\ \mathrm{U}[13]=20.0 ; & / * \mathrm{U}[14] & =\text { Right Trailing Edge Flap } & * / \\ \} & & & \\ & & & \\ \text { DHTCMD_L }=\mathrm{U}[0] & ; / * \mathrm{U}[1] & =\text { Left Stabilator } & * / \\ \text { DHTCMD_R }=\mathrm{U}[1] & ; / * \mathrm{U}[2] & =\text { Right Stabilator } & * / \\ \text { AILCMD_L }=\mathrm{U}[2] & ; / * \mathrm{U}[3] & =\text { Left Aileron } & * / \\ \text { AILCMD_R }=\mathrm{U}[3] & ; / * \mathrm{U}[4] & =\text { Right Aileron } & * / \\ \text { CANCMD_L }=\mathrm{U}[4] & ; / * \mathrm{U}[5] & =\text { Left Canard } & * / \\ \text { CANCMD_R }=\mathrm{U}[5] & ; / * \mathrm{U}[6] & =\text { Right Canard } & * / \\ \text { RUDCMD_L }=\mathrm{U}[6] & ; / * \mathrm{U}[7] & =\text { Left Rudder } & * / \\ \text { RUDCMD_R }=\mathrm{U}[7] & ; / * \mathrm{U}[8] & =\text { Right Rudder } & * / \\ \text { DPVCMD_L }=\mathrm{U}[8] & ; / * \mathrm{U}[9] & =\text { Left Pitch Vectoring } & * / \\ \text { DPVCMD_R }=\mathrm{U}[9] & ; / * \mathrm{U}[10] & =\text { Right Pitch Vectoring } & * / \\ \text { DYVCMD_L }=\mathrm{U}[10] & ; / * \mathrm{U}[11] & =\text { Left Yaw Vectoring } & * / \\ \text { DYVCMD_R }=\mathrm{U}[11] & ; / * \mathrm{U}[12] & =\text { Left Yaw Vectoring } & * / \\ \text { DTFCMD_L }=\mathrm{U}[12] & ; / * \mathrm{U}[13] & =\text { Left Trailing Edge Flap } & * / \\ \text { DTFCMD_R }=\mathrm{U}[13] & ; / * \mathrm{U}[14] & =\text { Right Trailing Edge Flap } & * /\end{array}$

/* Set initial throttle levels*/

PLAL $=100.0$

PLAR $=100.0 ;$

constants_();

/* Initialize the initial conditions of the aircraft */

$\begin{array}{lll}\text { VRW } & = & 600.0 ; \\ \text { STANDARD_DAY } & = & \text { TRUE; } \\ \text { ALT } & = & 10000.0 ; \\ \text { ALT_PRESSURE } & = & 10000.0 ; \\ \text { THET } & = & 0.0 ; \\ \text { PHI } & = & 0.0 ; \\ \text { PSI } & = & 0.0 ; \\ \text { ALFA } & = & 0.0 ; \\ \text { BETA } & = & 0.0 ; \\ \text { GAMV } & = & 0.0 ; \\ \text { GAMH } & = & 0.0 ; \\ \text { PBDEG } & = & 0.0 ; \\ \text { QBDEG } & = & 0.0 ; \\ \text { RBDEG } & = & 0.0 ;\end{array}$


Kevin R. Scalera

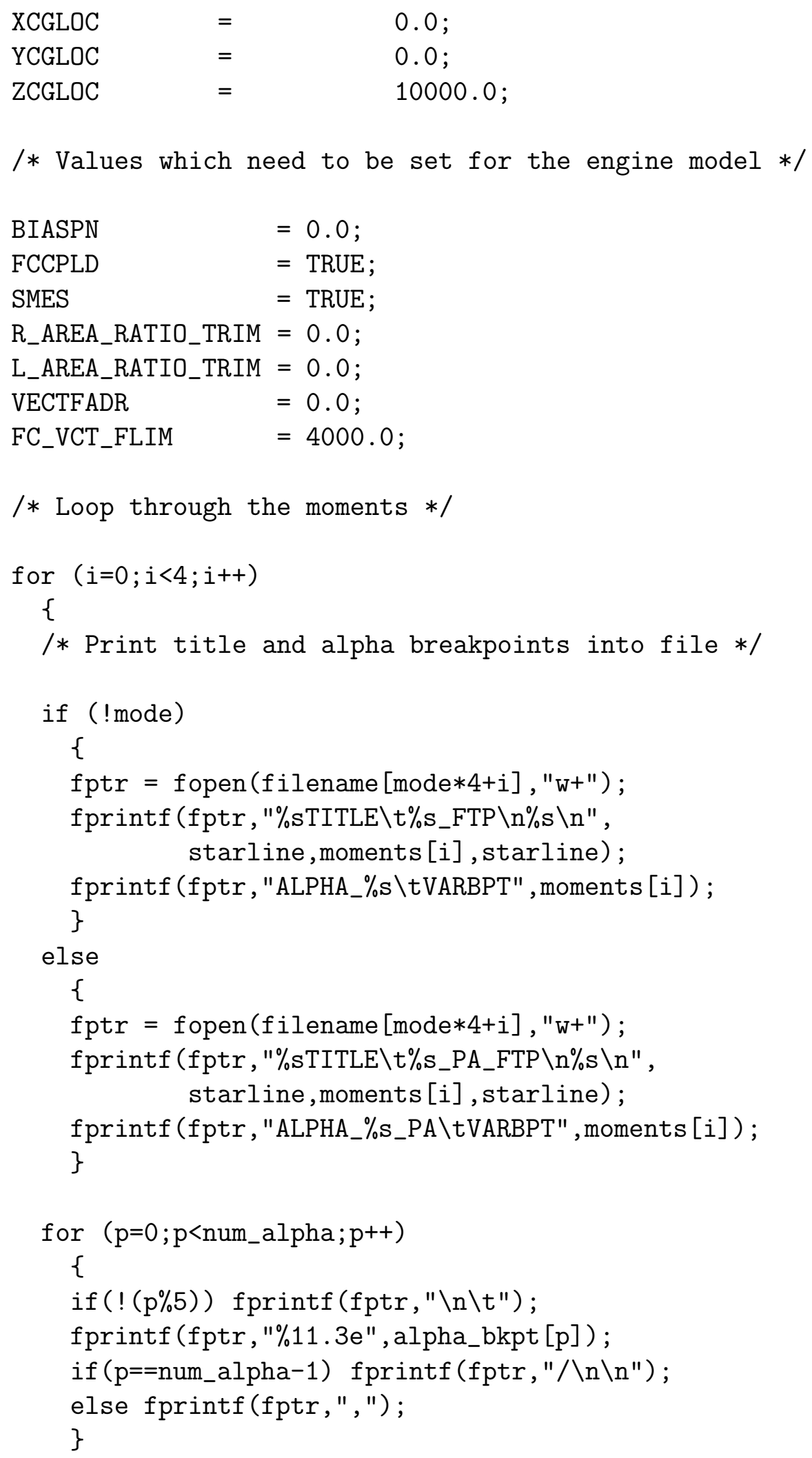




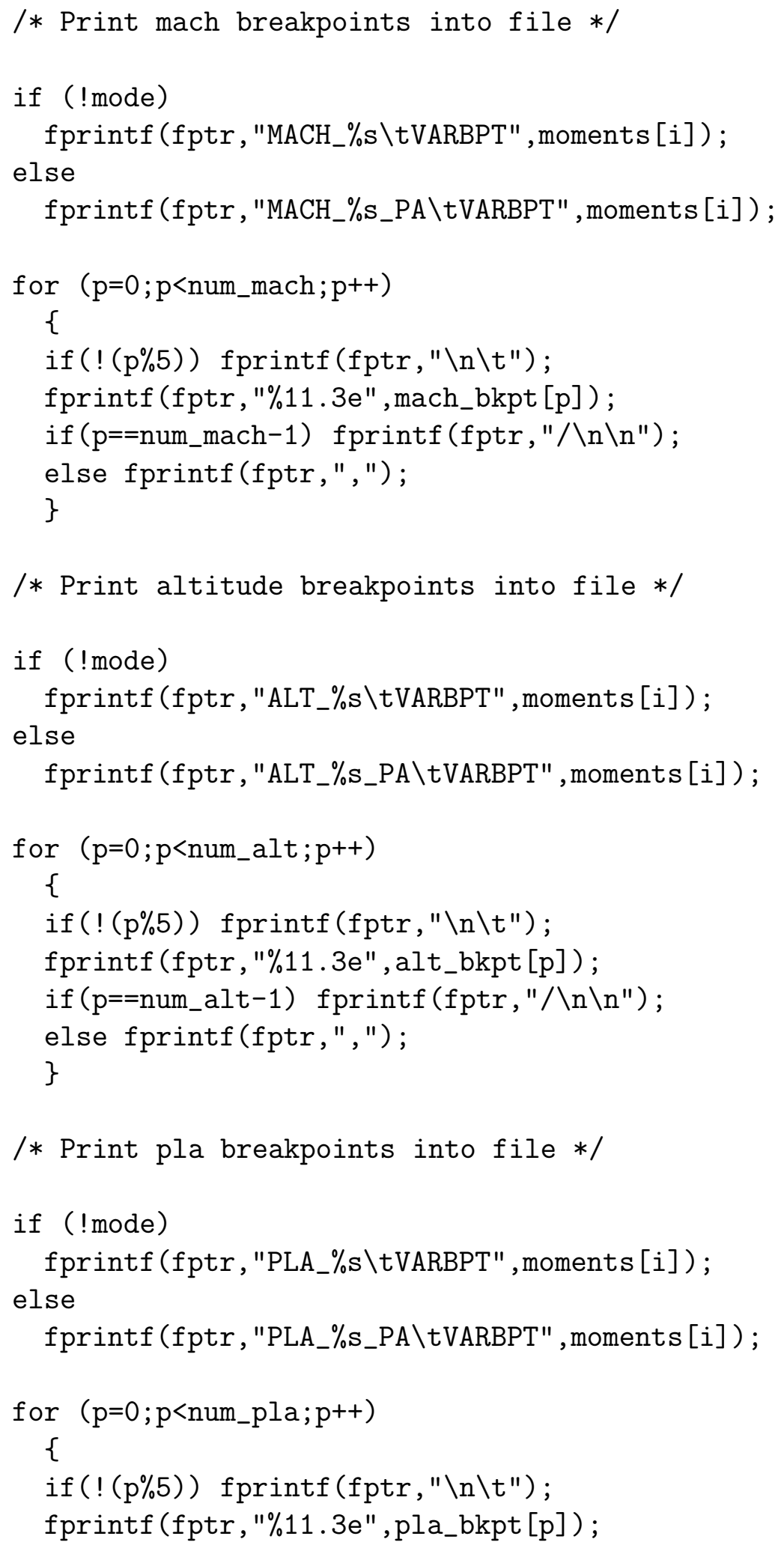




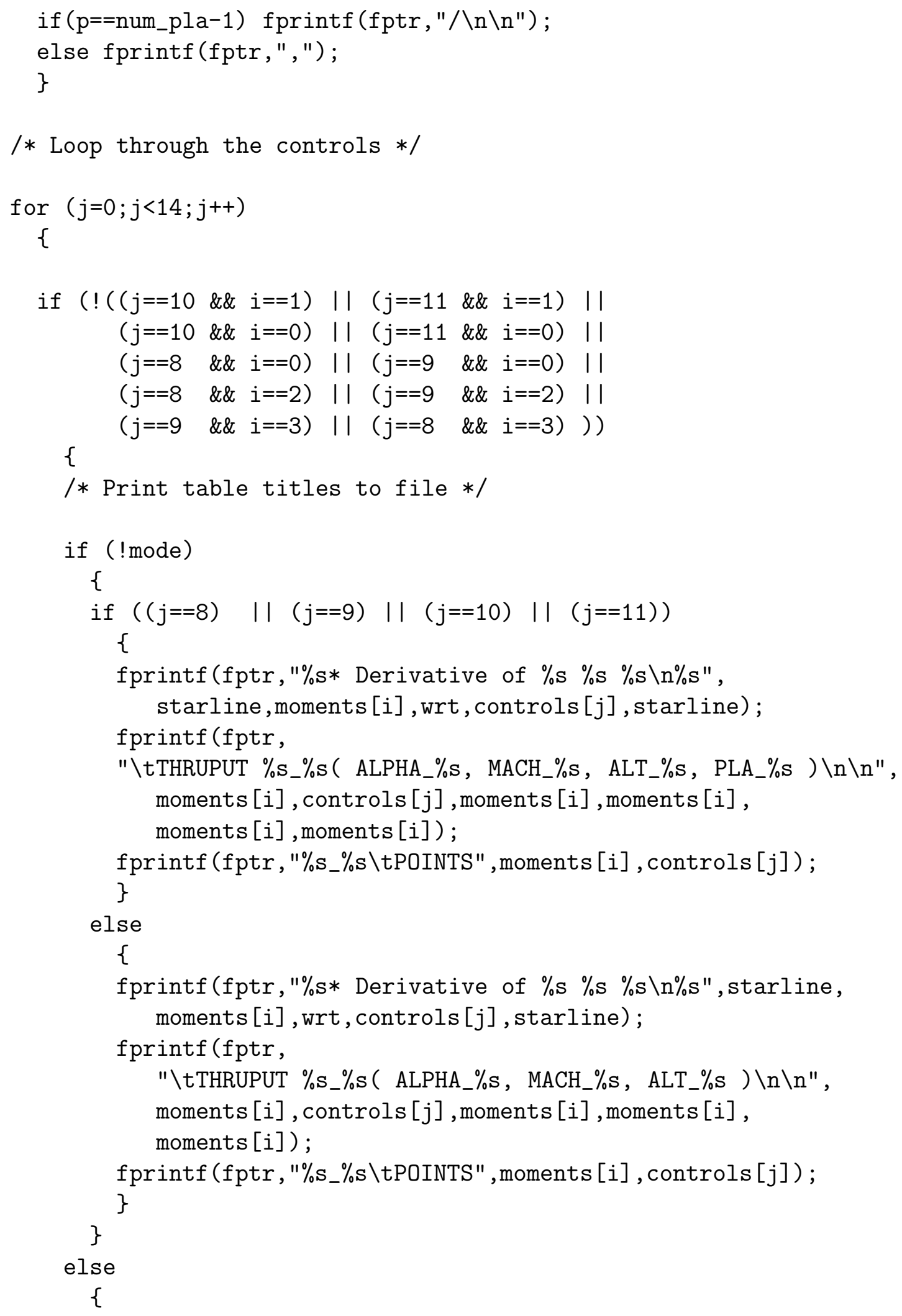




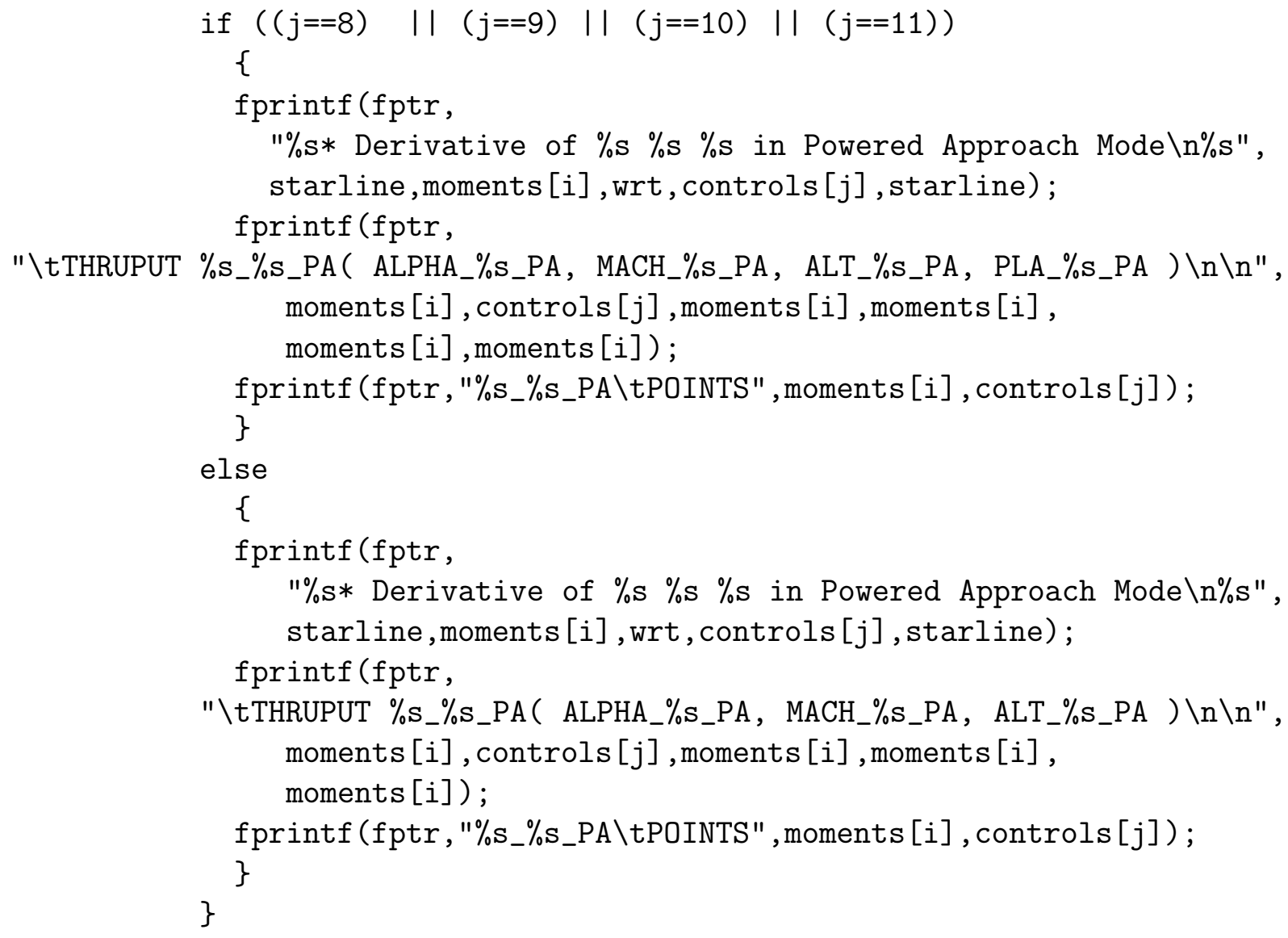




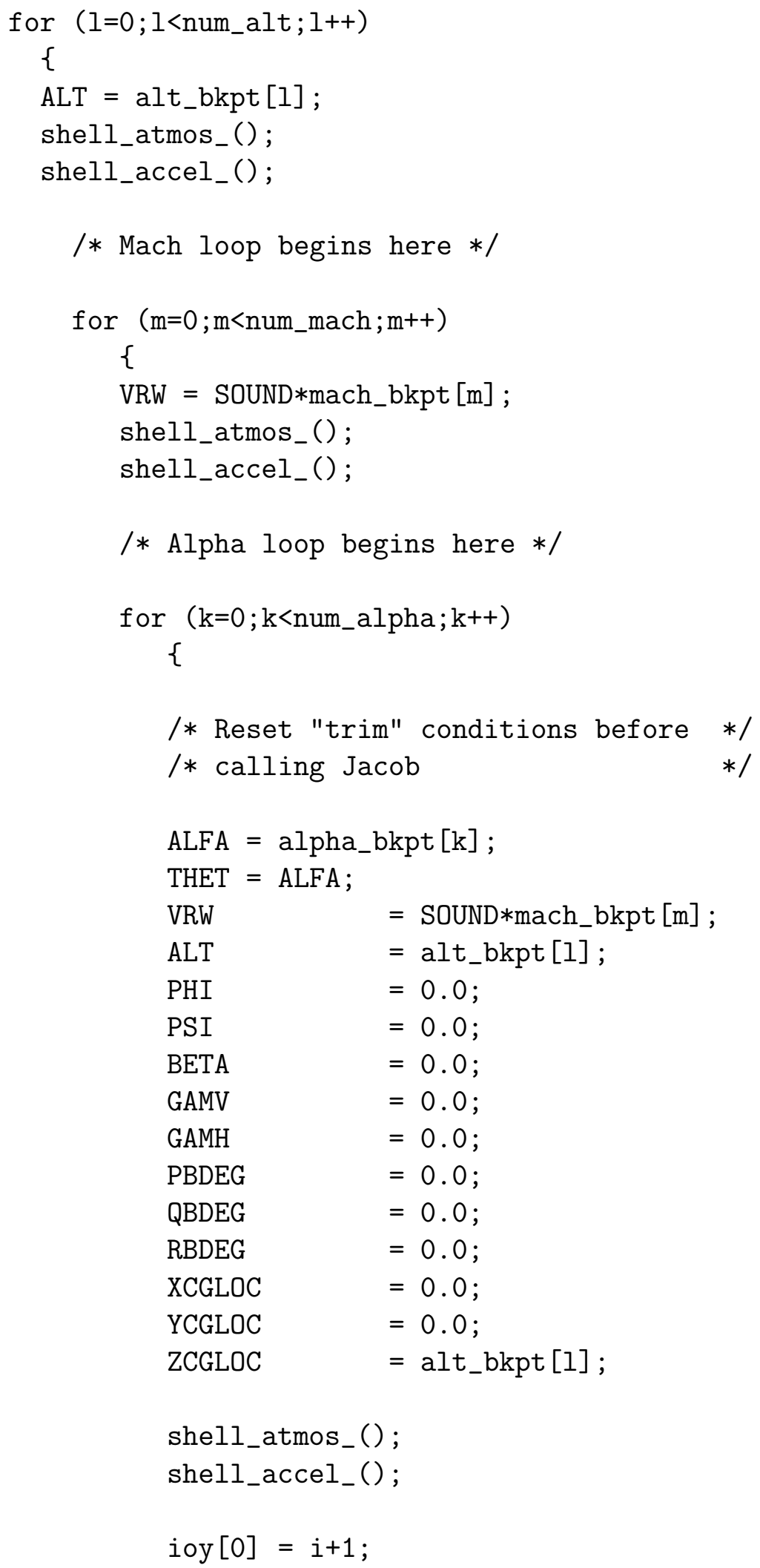




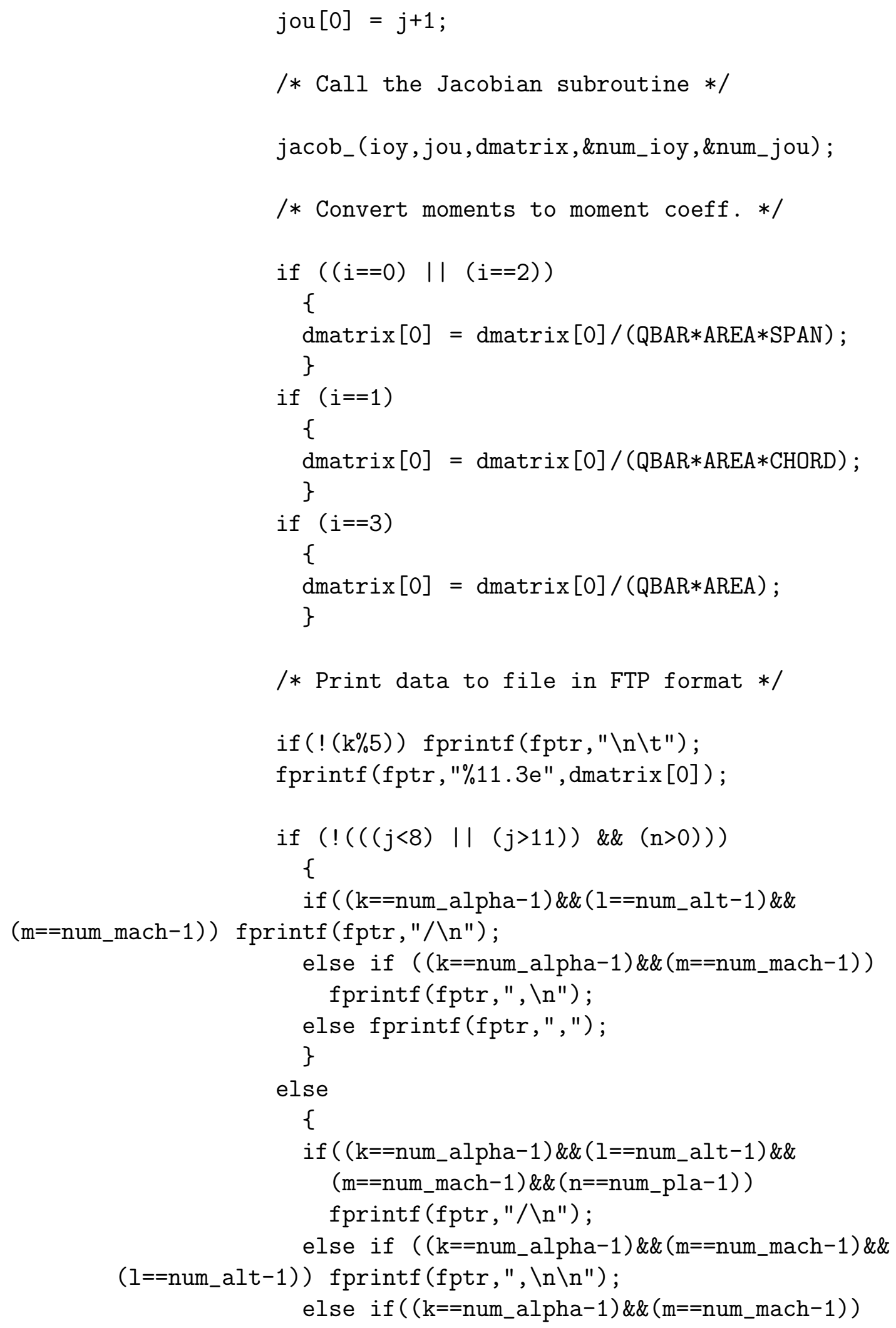




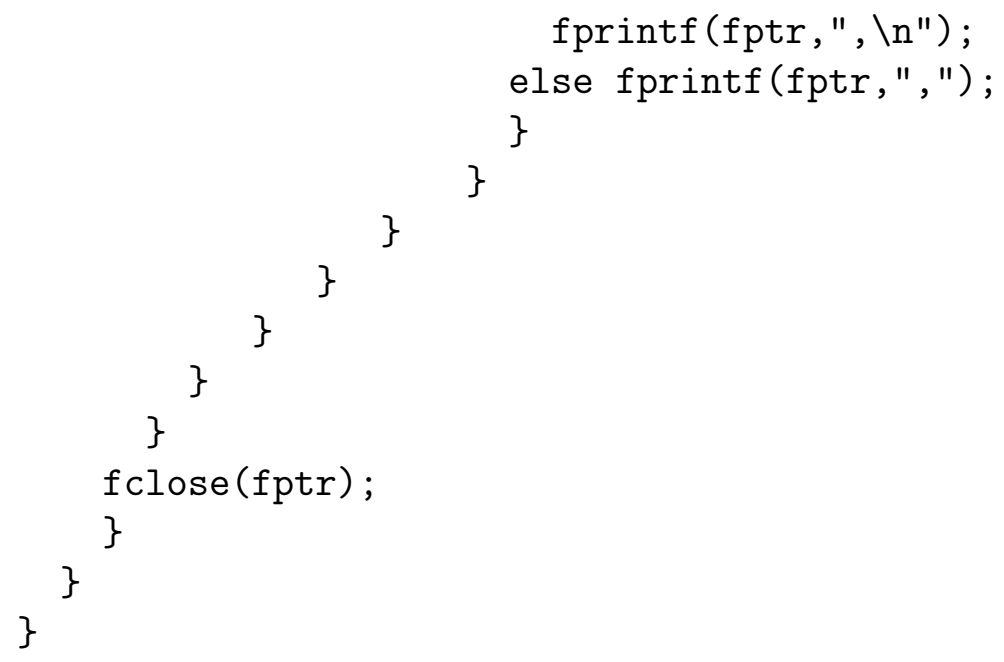




\section{B.2 Subroutine Jacob.f}

\section{B.2.1 Description}

Subroutine JACOB calculates the Jacobian matrices for the set of aircraft nonlinear state equations. This code is taken from primarily from reference 27 with small modifications. Although subroutine JACOB has the capability to calculate all four state matrices, A, B, $\mathbf{C}$ and $\mathbf{D}$, in this work, only the control effectiveness matrix, $\mathbf{B}$, is needed.

As stated in reference 27, "The linearization algorithm chooses smaller and smaller perturbations in the independent variable and compares three successive approximations to the particular partial derivative. If these approximations agree within a certain tolerance, then the size of the perturbation is reduced to determine if an even smaller tolerance can be satisfied." The perturbations are performed by the subroutine YDU, discussed in section B.3. If the algorithm does not attain the desired tolerance, the user is presented with a list of the successive approximations, from which he is asked to decide upon the partial derivative. This user input is subjective, but in general, one can perceive a trend in the successive approximations and confidently enter a solution that is "close" to the correct partial derivative.

Subroutine JACOB has been coded to be compatible with the standard CASTLE architecture. Common blocks and equivalence statements are automatically created using software associated with CASTLE. Variable declarations for inputs and outputs are listed in the "DECLARATION SECTION". Variables defined as "OTHER LOCALS" are considered to be temporary variables and do not have definitions associated with them. 


\section{B.2.2 Code}

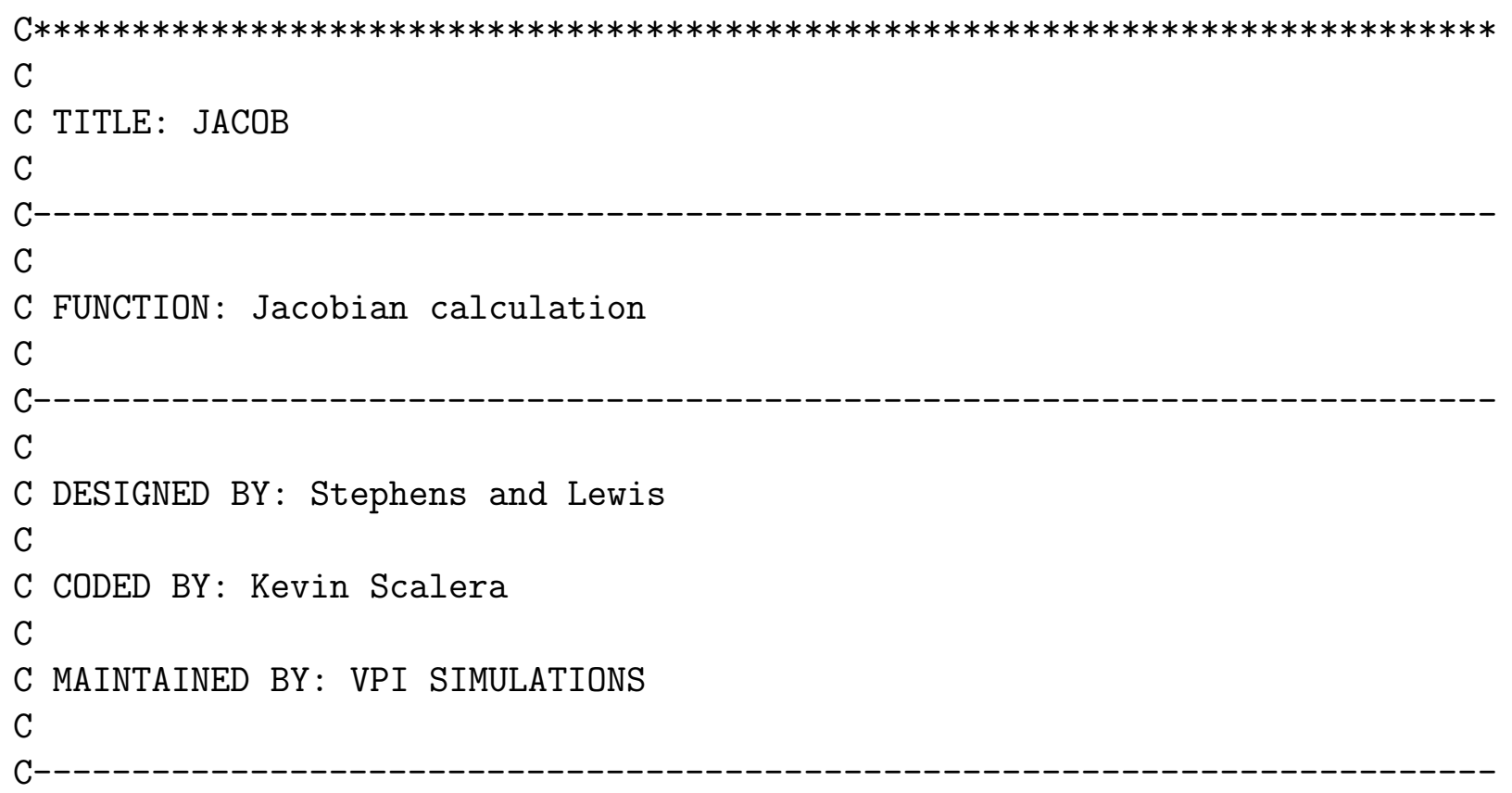

C

C MODIFICATION HISTORY:

$\mathrm{C}$

C DATE PURPOSE BY

$\mathrm{C}====$ ======= ==

C

C-

C

C GLOSSARY

$\mathrm{C}=======$

C

C ASSIGNMENTS:

C

C NONE

C

$\mathrm{C}$

C

C INPUTS:

C

C IMODE Sim. mode: -2=init, $-1=$ reset, $0=$ hold, 1=ru --------

C M Number of control effectors to be used --------

C U(1) Vector of allocated controls C 


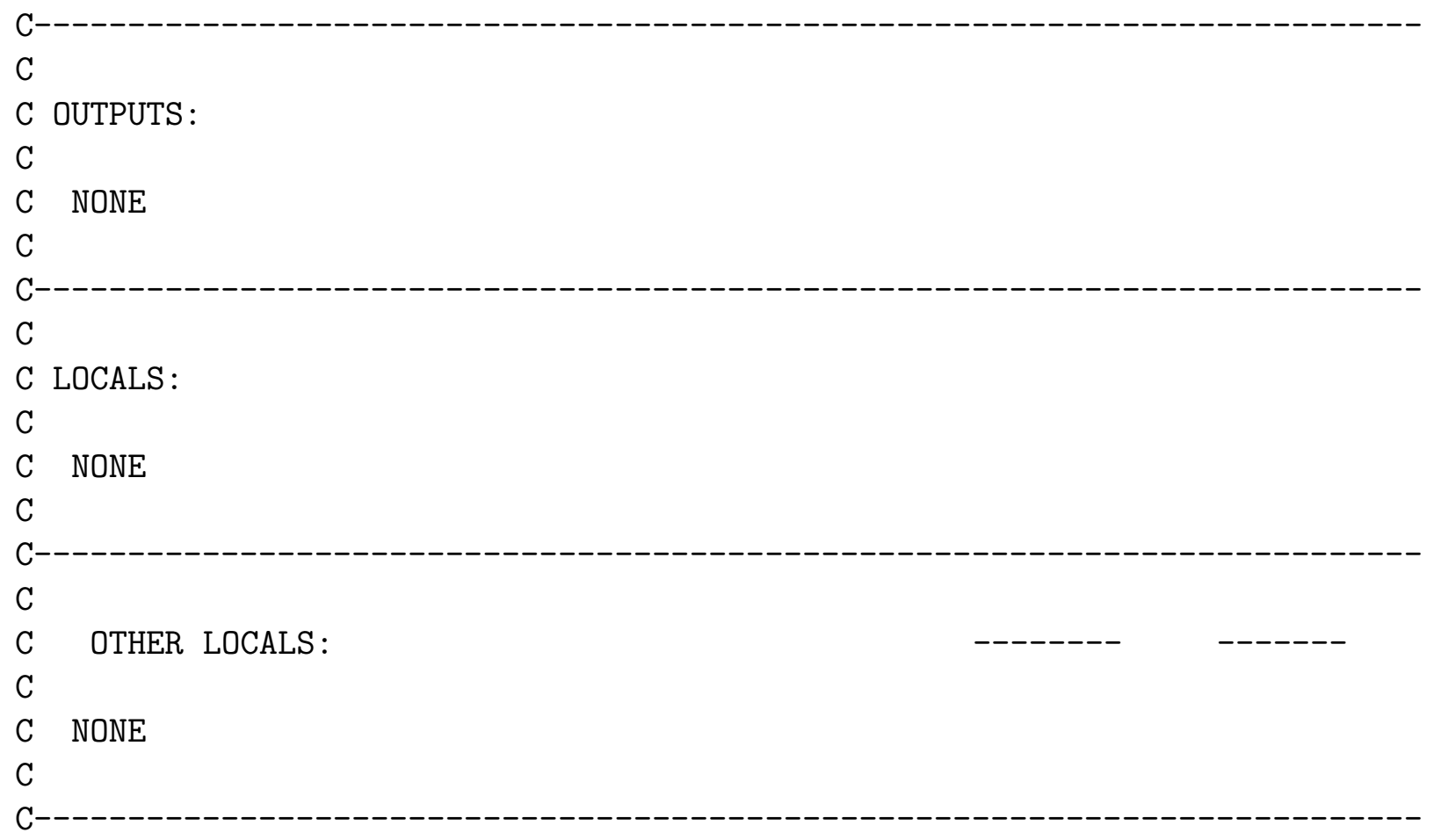

SUBROUTINE JACOB（IOY, JOU,DMATRIX, NUM_IOY,NUM_JOU）

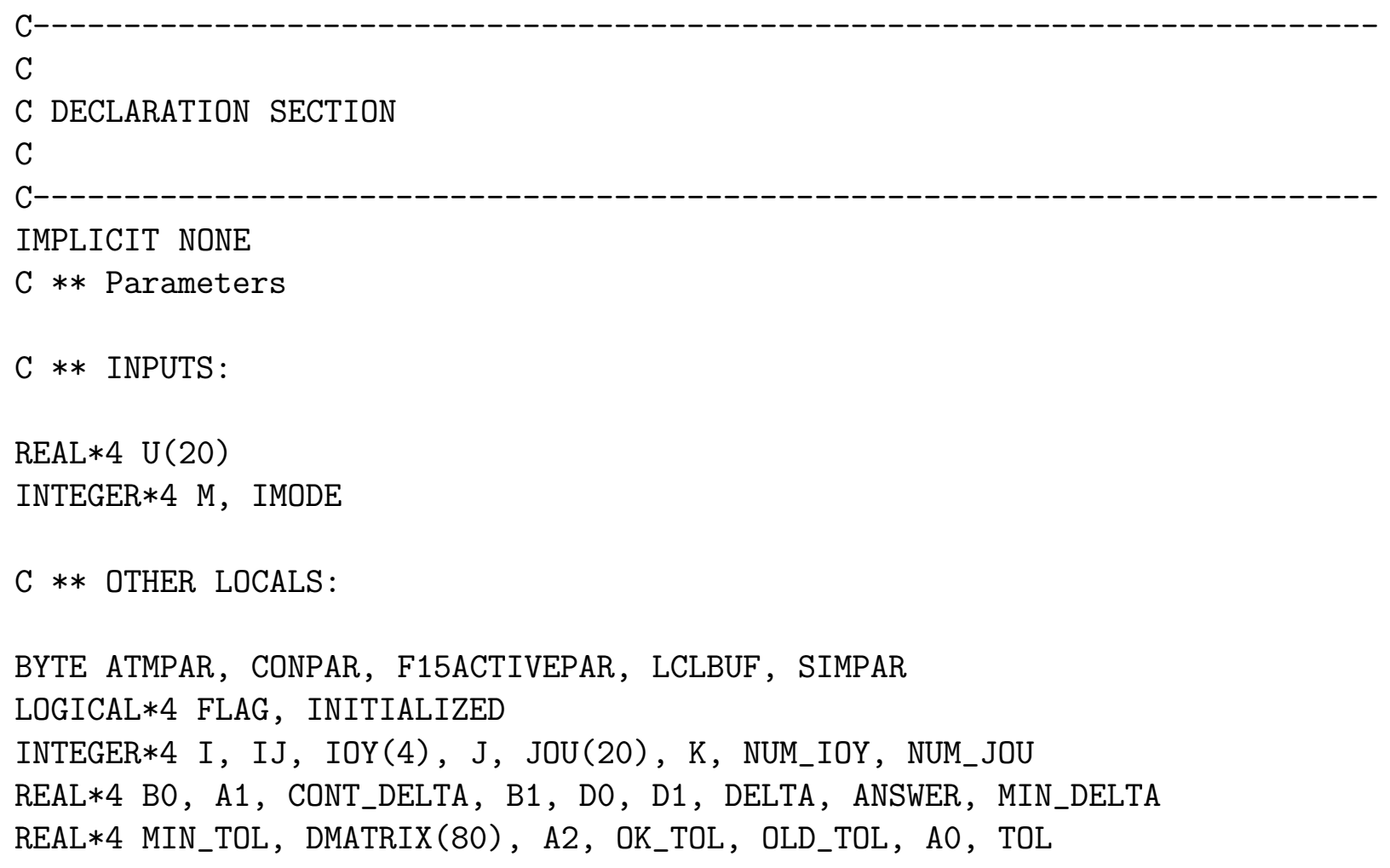


REAL*8 DDU, YDU

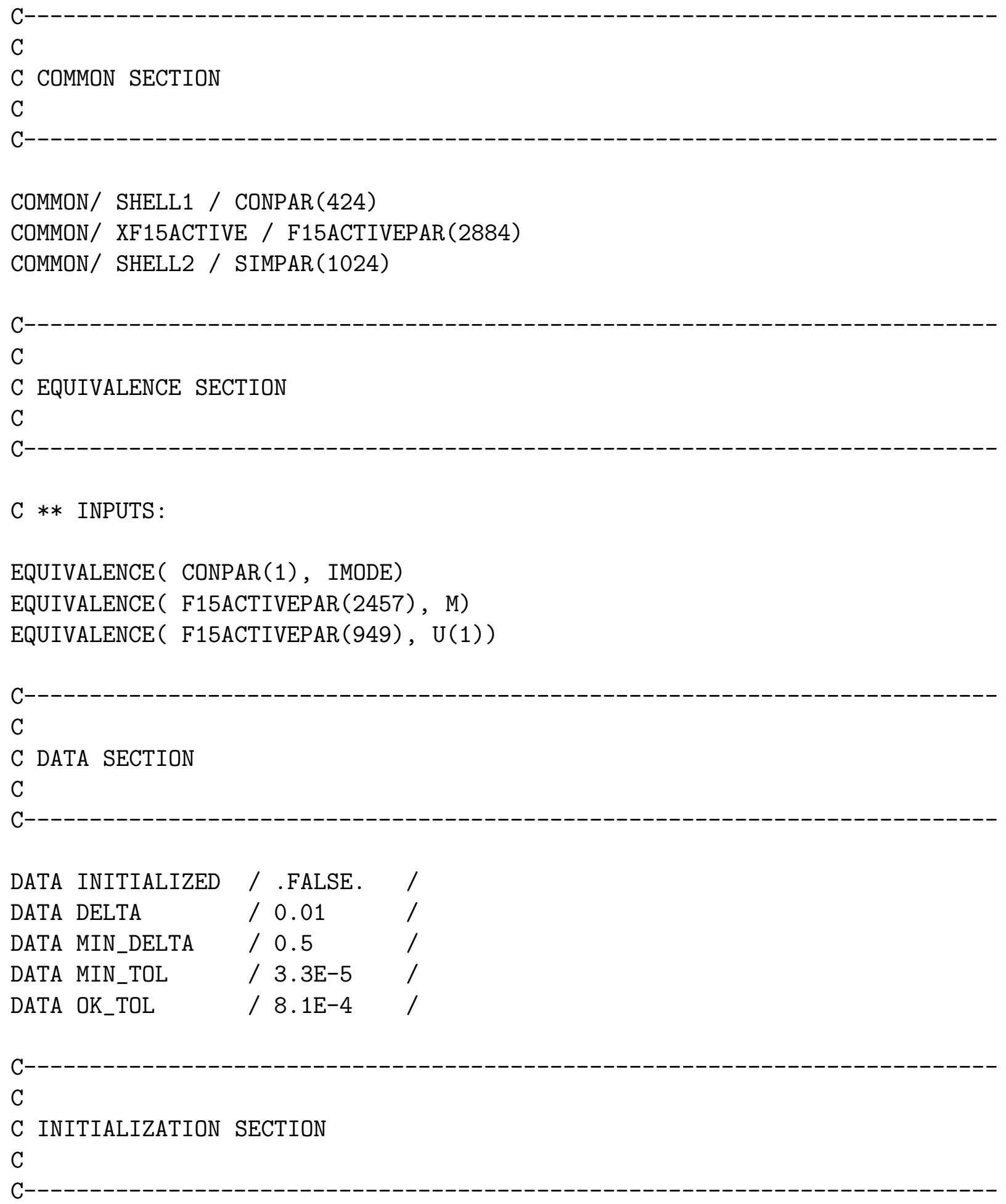


IF（（IMODE.LE.-2）.OR. .NOT.INITIALIZED ) THEN

\section{ENDIF}

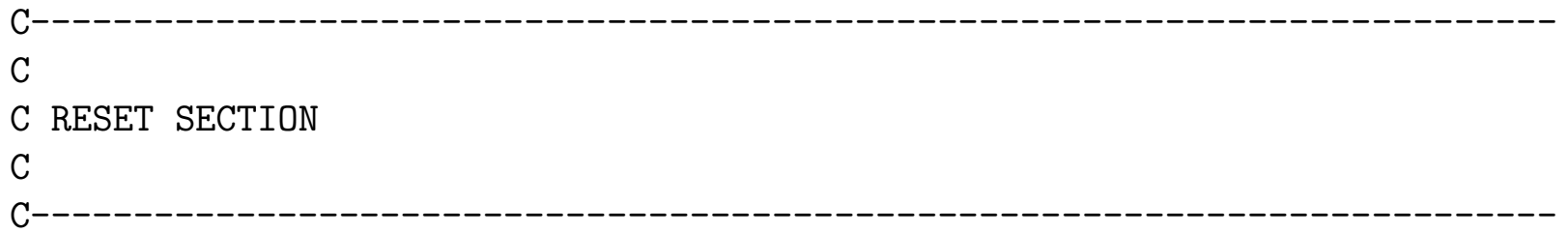

IF ((IMODE.LE.-1).OR. (.NOT.Initialized)) THEN Initialized $=$. TRUE.

END IF

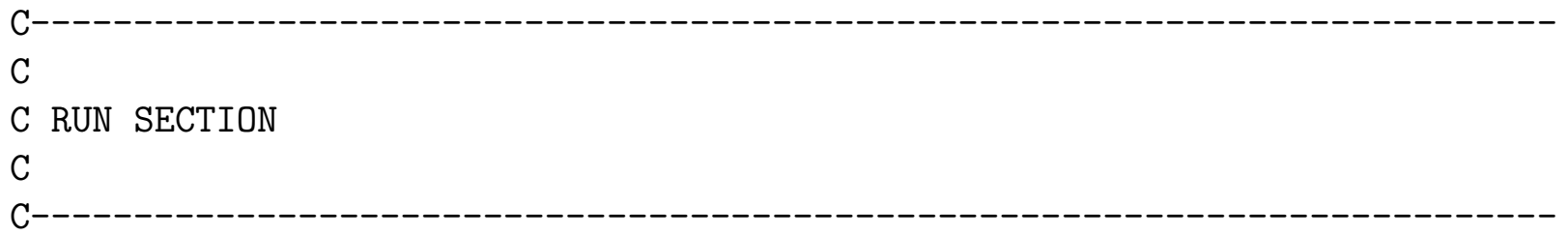

$\mathrm{IJ}=1$

DO $40 \mathrm{~J}=1$,NUM_JOU

CONT_DELTA $=\operatorname{AMAX1}(\operatorname{ABS}(\operatorname{DELTA} * \mathrm{U}(\mathrm{JOU}(\mathrm{J})))$, MIN_DELTA $)$

DO $40 \mathrm{I}=1$,NUM_IOY

FLAG $=$.FALSE .

1

TOL $=0.1$

OLD_TOL $=$ TOL

$\mathrm{DDU}=\mathrm{DBLE}($ CONT_DELTA $)$

$\mathrm{A} 2=0.0$

$\mathrm{A} 1=0.0$

$\mathrm{AO}=0.0$

$\mathrm{B} 1=0.0$

$\mathrm{BO}=0.0$

$\mathrm{D} 1=0.0$

DO $=0.0$

$\operatorname{WRITE}(*$, '(/6X , A12, 8X , A5) ') 'Perturbation' , 'Slope'

DO $20 \mathrm{~K}=1,18$

$\mathrm{A} 2=\mathrm{A} 1$

$\mathrm{A} 1=\mathrm{A} 0$

$\mathrm{B} 1=\mathrm{B} 0$ 


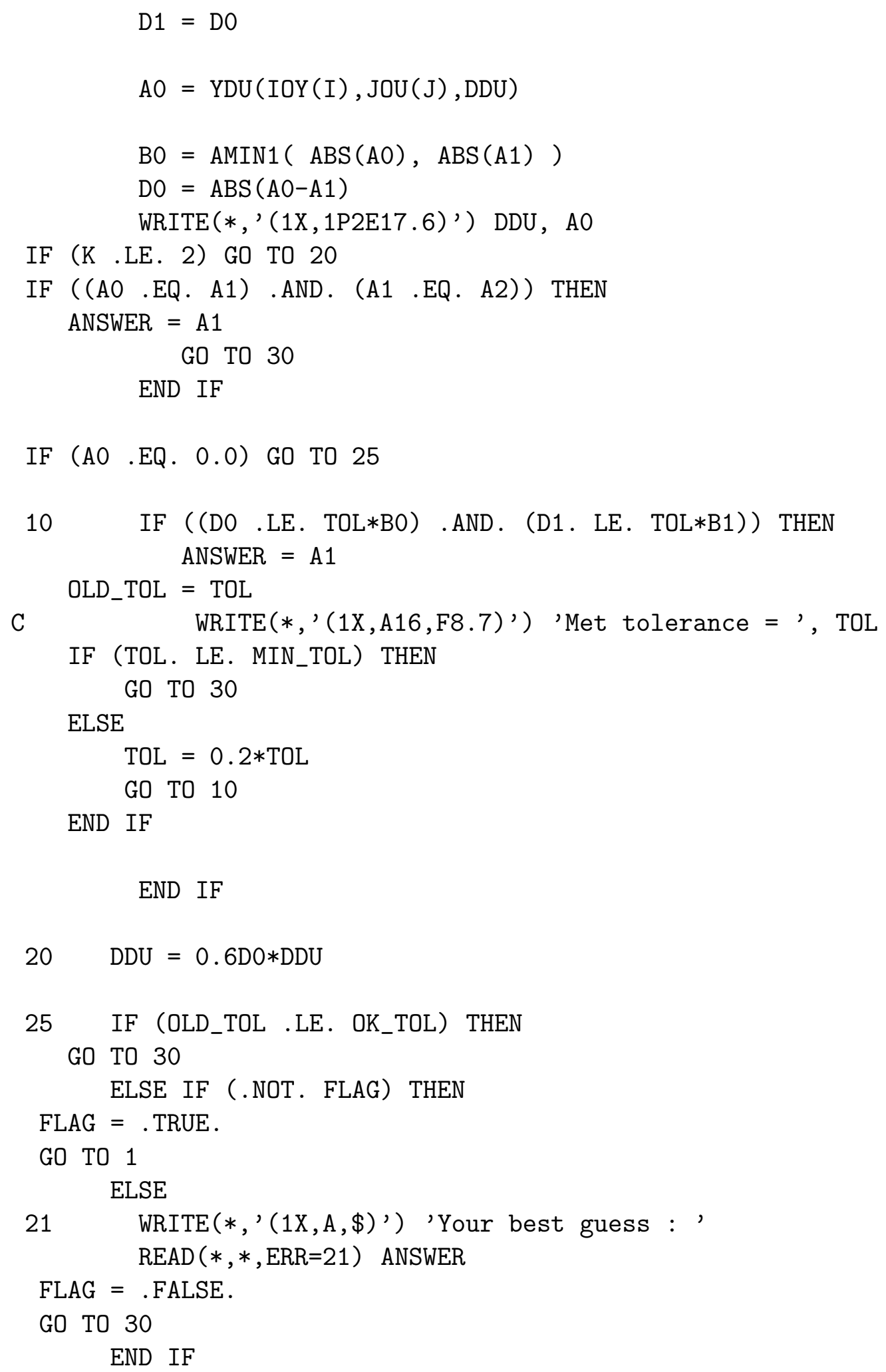


30 DMATRIX $(\mathrm{IJ})=$ ANSWER

$40 \quad \mathrm{IJ}=\mathrm{IJ}+1$

C

C END OF JACOB MODULE

C

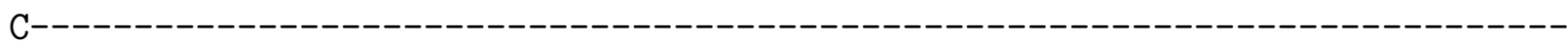

RETURN

END 


\section{B.3 Function Ydu.f}

\section{B.3.1 Description}

Function YDU is a double precision function called by subroutine JACOB. The purpose of YDU is to perturb each of the control surfaces and determine the resultant effect on each of the moments. YDU calls a series of CASTLE subroutines that ultimately integrate the set of aircraft nonlinear equations of motion for the given flight condition. The controls are first perturbed in the negative direction and then in the positive direction. The difference in the resulting moments can be used in conjunction with the perturbation size to find the desired approximate partial derivative. The partial derivative is taken about the zero deflection point for all controls in the flaps-up configuration. In the flaps-down configuration, since the flaps are drooped to 20 degrees, the partial derivative is taken about this deflection point.

\section{B.3.2 Code}

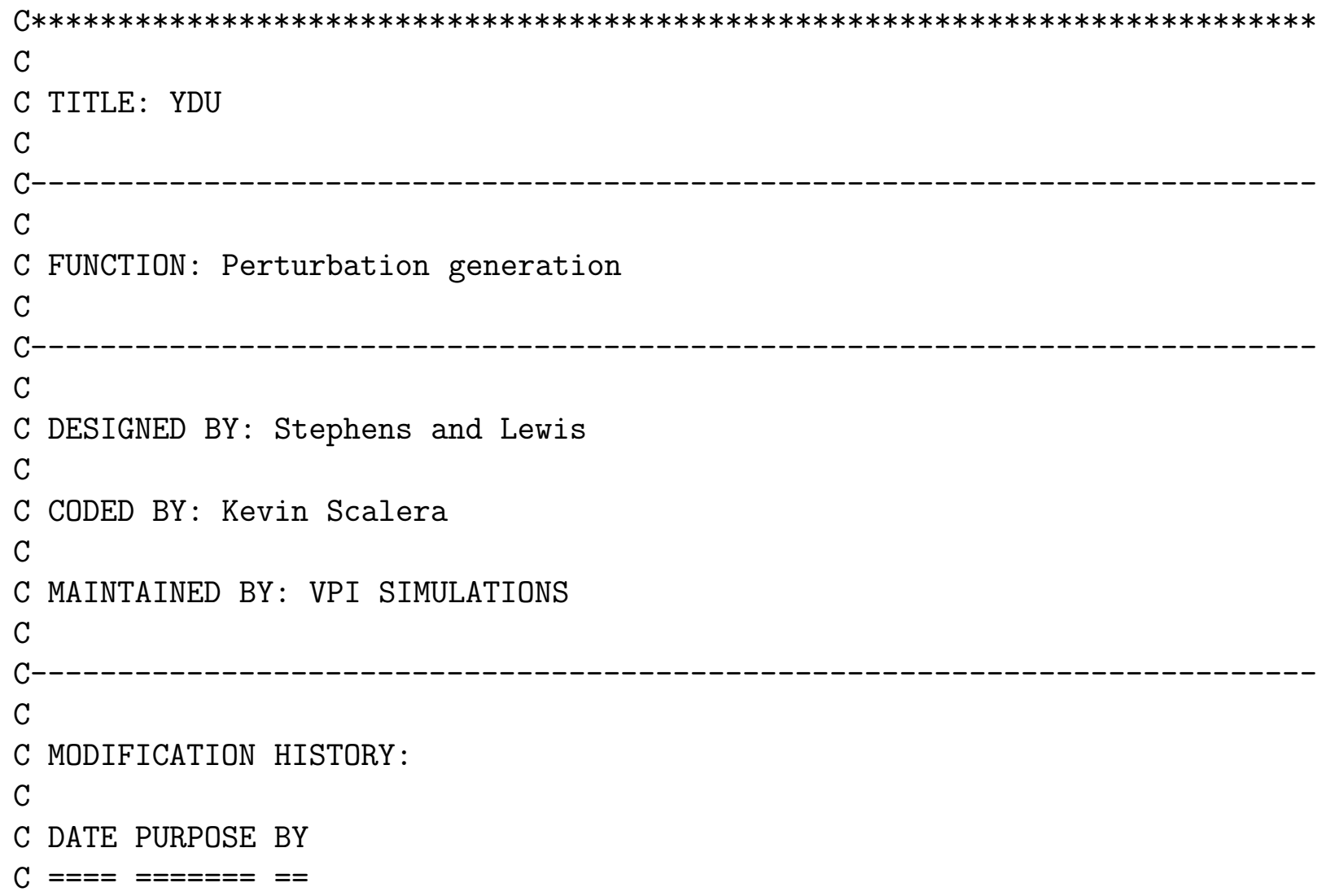




\begin{tabular}{|c|c|c|c|c|}
\hline \multicolumn{5}{|l|}{$\mathrm{C}$} \\
\hline \multicolumn{5}{|l|}{ C } \\
\hline \multicolumn{5}{|c|}{ C GLOSSARY } \\
\hline \multicolumn{5}{|c|}{$\mathrm{C}========$} \\
\hline \multicolumn{5}{|l|}{$\mathrm{C}$} \\
\hline \multirow{2}{*}{\multicolumn{5}{|c|}{ C ASSIGNMENTS: }} \\
\hline & & & & \\
\hline \multirow{2}{*}{\multicolumn{5}{|c|}{$\begin{array}{ll}\text { C NONE } \\
\text { C }\end{array}$}} \\
\hline & \multicolumn{4}{|c|}{$\mathrm{C}$} \\
\hline \multicolumn{5}{|c|}{ 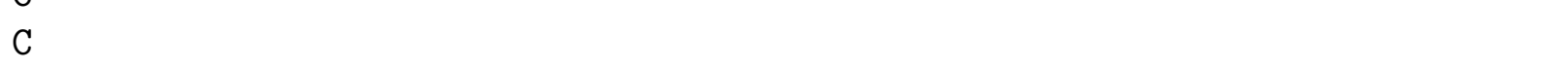 } \\
\hline \multicolumn{5}{|c|}{ C INPUTS: } \\
\hline $\mathrm{C}$ & & & & \\
\hline $\mathrm{C}$ & AILCMD_L & Left aileron command & Deg & --- \\
\hline $\mathrm{C}$ & AILCMD_R & Right aileron command & Deg & ------- \\
\hline $\mathrm{C}$ & AREA & Wing Area & FT2 & $\mathrm{N} / \mathrm{A}$ \\
\hline $\mathrm{C}$ & CANCMD_L & Left canard command & $\operatorname{deg}$ & ------- \\
\hline $\mathrm{C}$ & CANCMD_R & Right canard command & Deg & ------- \\
\hline $\mathrm{C}$ & CHORD & Mean Aerodynamic Chord & FT & $\mathrm{N} / \mathrm{A}$ \\
\hline $\mathrm{C}$ & DHTCMD_L & Left horizontal tail command & Deg & ------- \\
\hline $\mathrm{C}$ & DHTCMD_R & Right horizontal tail command & Deg & ------- \\
\hline $\begin{array}{l}\mathrm{C} \\
\mathrm{C} !\end{array}$ & DPVCMD_L & $\begin{array}{l}\text { Roll vectoring command left for long } \\
\text { control }\end{array}$ & -------- & --- \\
\hline $\begin{array}{l}\mathrm{C} \\
\mathrm{C} !\end{array}$ & DPVCMD_R & $\begin{array}{l}\text { Roll vectoring command right for long } \\
\text { control }\end{array}$ & -------- & -- \\
\hline $\mathrm{C}$ & DTFCMD_L & Left flap command & Deg & --- \\
\hline $\mathrm{C}$ & DTFCMD_R & Right flap command & Deg & --- \\
\hline $\mathrm{C}$ & DYVCMD_L & Yaw vectoring limit left for lat.-dir. & $\operatorname{deg}$ & ------- \\
\hline $\mathrm{C}$ & DYVCMD_R & Yaw vectoring limit right for lat.-dir. & $\operatorname{deg}$ & ------- \\
\hline $\mathrm{C}$ & FTY & Total Forces On A/c, Y-body & LB & RT \\
\hline $\mathrm{C}$ & IMODE & Sim. mode: $-2=$ init, $-1=$ reset, $0=$ hold, $1=r u$ & -------- & --- \\
\hline $\mathrm{C}$ & QBAR & Dynamic Pressure & LB/FT2 & $\mathrm{N} / \mathrm{A}$ \\
\hline $\mathrm{C}$ & RUDCMD_L & Left rudder command & Deg & ------- \\
\hline $\mathrm{C}$ & RUDCMD_R & Right rudder command & Deg & ------- \\
\hline $\mathrm{C}$ & SPAN & Wing Span & FT & $\mathrm{N} / \mathrm{A}$ \\
\hline $\mathrm{C}$ & TTL & Total Rolling Moment, X-body & FT-LB & RWD \\
\hline $\mathrm{C}$ & TTM & Total Pitching Moment, Y-body & FT-LB & ANU \\
\hline $\mathrm{C}$ & TTN & Total Yawing Moment, Z-body & FT-LB & ANR \\
\hline $\mathrm{C}$ & $\mathrm{U}(1)$ & Vector of allocated controls & -------- & ------- \\
\hline
\end{tabular}




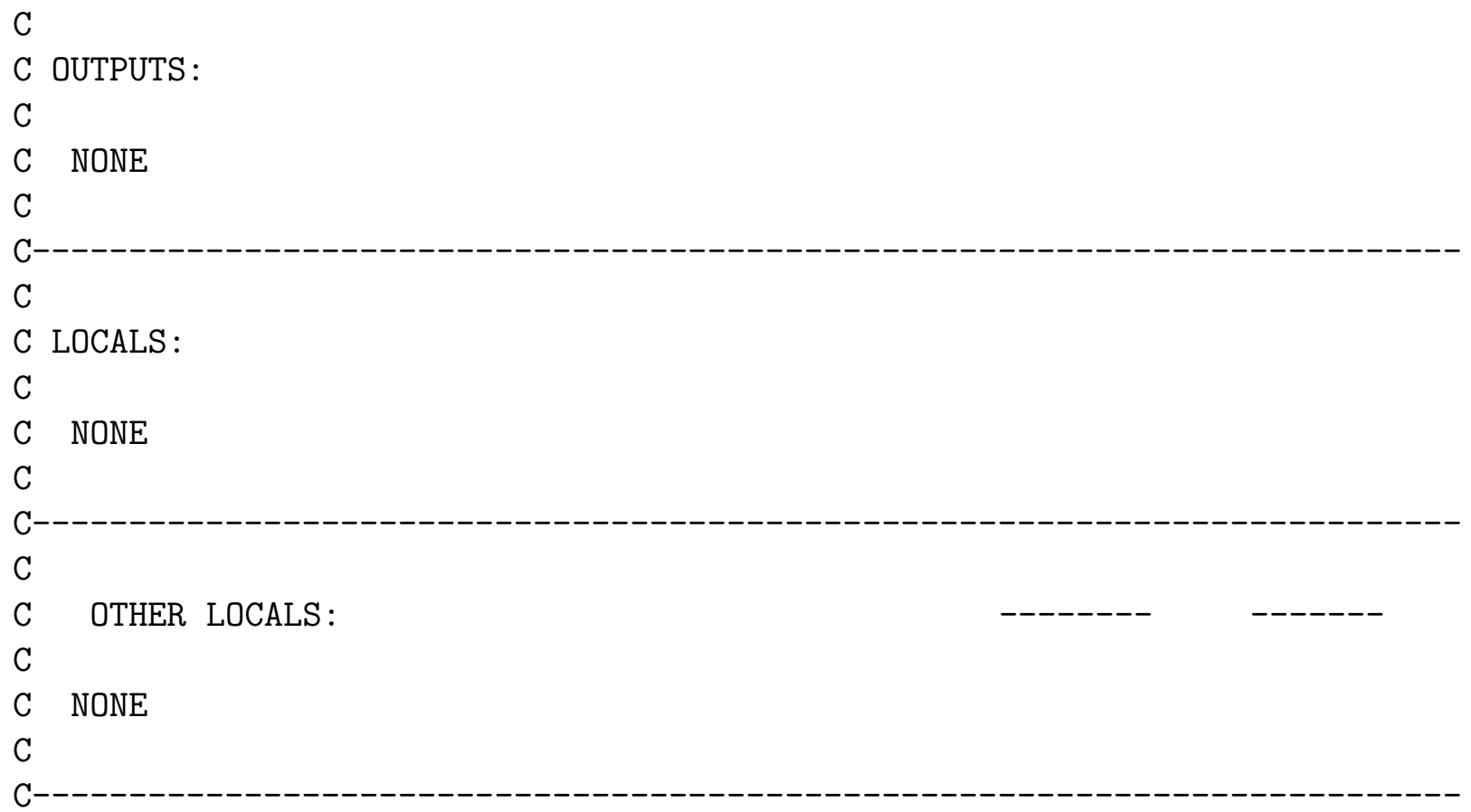

FUNCTION YDU(OUT_NUM, CONT_NUM, DDU)

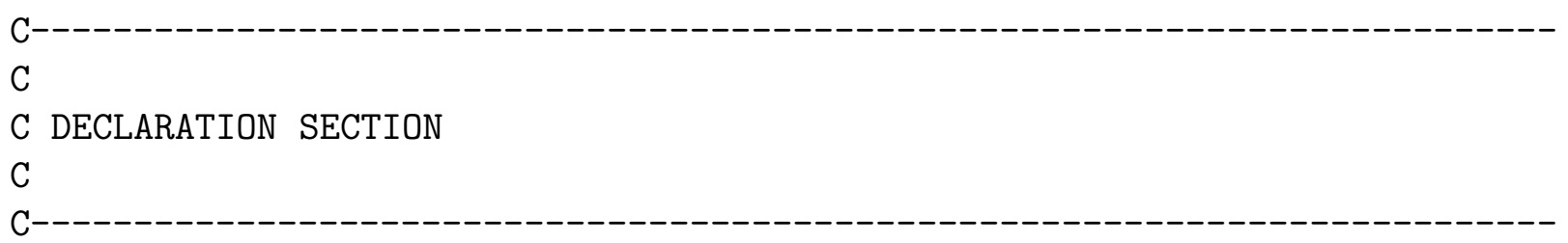

IMPLICIT NONE

C ** Parameters

$\mathrm{C} * *$ INPUTS:

INTEGER $* 4$ IMODE

REAL*4 AILCMD_R, AREA, CANCMD_L, CANCMD_R, CHORD, DHTCMD_L

REAL*4 DHTCMD_R, DPVCMD_L, DPVCMD_R, DTFCMD_L, DTFCMD_R

REAL*4 DYVCMD_L, DYVCMD_R, FTY, AILCMD_L, QBAR, RUDCMD_L

REAL*4 RUDCMD_R, SPAN, TTL, TTM, TTN, U(20)

C ** OTHER LOCALS :

BYTE CONPAR, F15ACTIVEPAR, LCLBUF, SIMPAR

INTEGER $* 4$ OUT_NUM, CONT_NUM

REAL*4 MOMENTS (4) 
REAL*8 DDU, TEMP_CONT, YD1, YD2, YDU

LOGICAL $* 4$ INITIALIZED

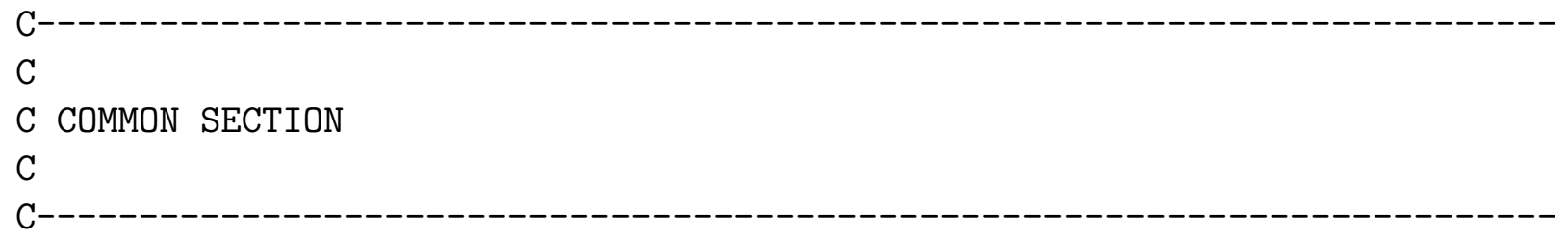

COMMON/ XF15ACTIVE / F15ACTIVEPAR(3452)

COMMON/ SHELL2 / $\operatorname{SIMPAR}(1024)$

COMMON / SHELL1 / CONPAR (424)

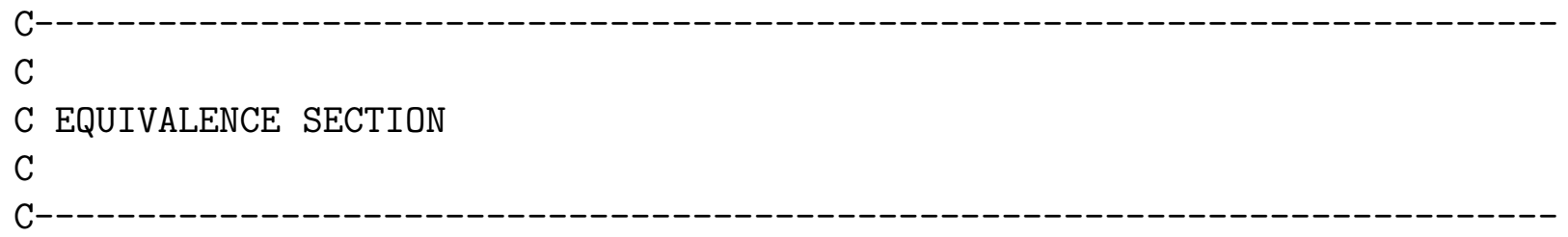

C ** INPUTS:

EQUIVALENCE( F15ACTIVEPAR (245), AILCMD_L) EQUIVALENCE( F15ACTIVEPAR (241), AILCMD_R) EQUIVALENCE( SIMPAR (557), AREA) EQUIVALENCE( F15ACTIVEPAR(277), CANCMD_L) EQUIVALENCE( F15ACTIVEPAR (273), CANCMD_R) EQUIVALENCE( SIMPAR (565), CHORD)

EQUIVALENCE ( F15ACTIVEPAR (253), DHTCMD_L) EQUIVALENCE ( F15ACTIVEPAR (249), DHTCMD_R) EQUIVALENCE ( F15ACTIVEPAR (233), DPVCMD_L) EQUIVALENCE ( F15ACTIVEPAR (237), DPVCMD_R) EQUIVALENCE( F15ACTIVEPAR (261), DTFCMD_L) EQUIVALENCE ( F15ACTIVEPAR (257), DTFCMD_R) EQUIVALENCE ( F15ACTIVEPAR (213), DYVCMD_L) EQUIVALENCE( F15ACTIVEPAR (217), DYVCMD_R) EQUIVALENCE ( SIMPAR (433), FTY) EQUIVALENCE ( CONPAR(1), IMODE) EQUIVALENCE( SIMPAR (549), QBAR) EQUIVALENCE( F15ACTIVEPAR(269), RUDCMD_L) EQUIVALENCE( F15ACTIVEPAR (265), RUDCMD_R) EQUIVALENCE ( SIMPAR (561), SPAN) EQUIVALENCE( SIMPAR(505), TTL) 


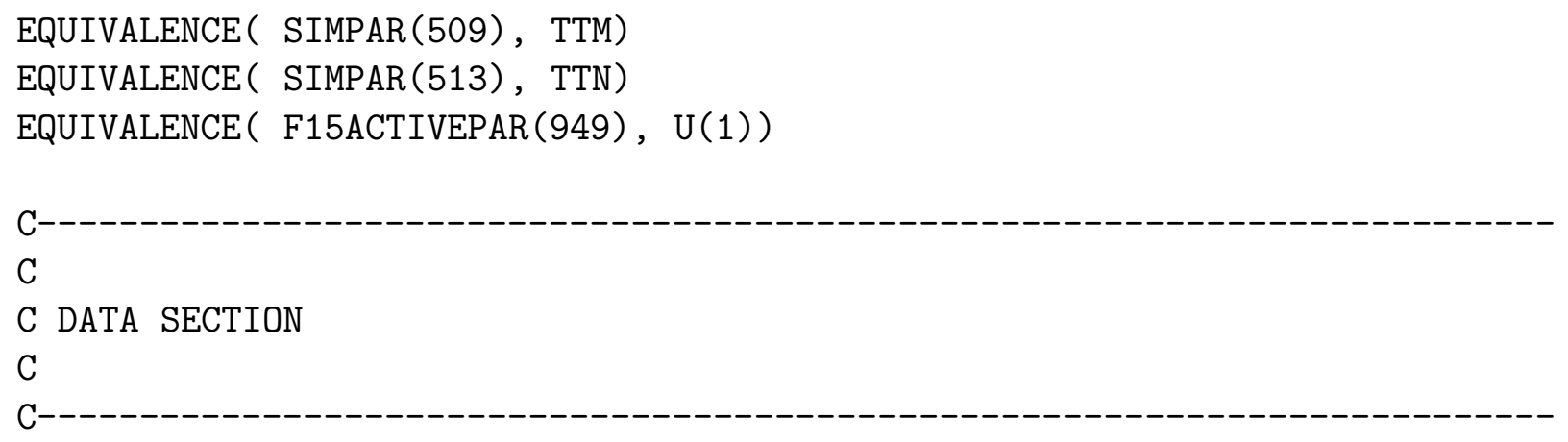

\section{DATA INITIALIZED / .FALSE. /}

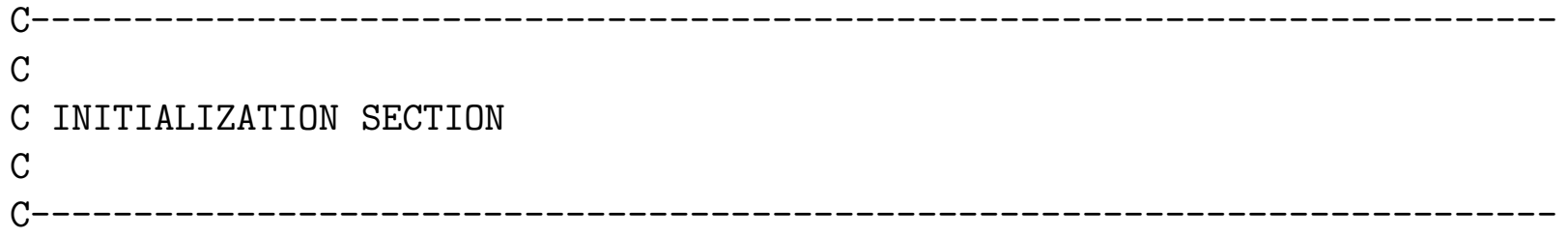

IF ( (IMODE.LE. -2) .OR. .NOT. INITIALIZED ) THEN

ENDIF

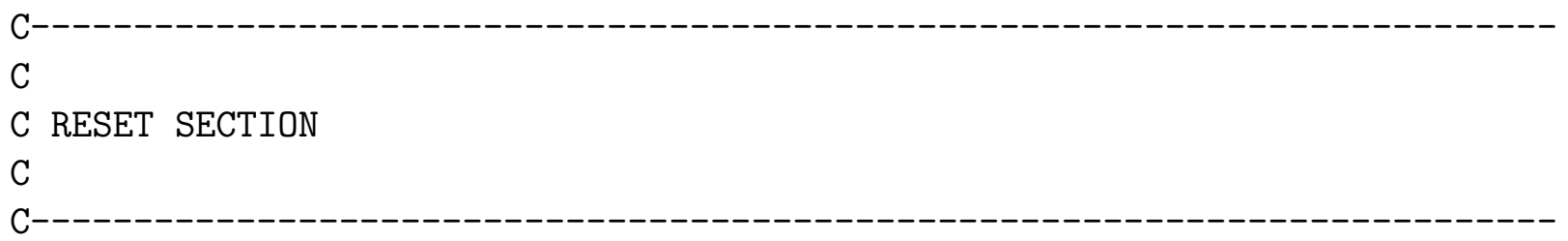

IF ((IMODE.LE.-1).OR. (.NOT.Initialized)) THEN

Initialized $=$. TRUE.

END IF

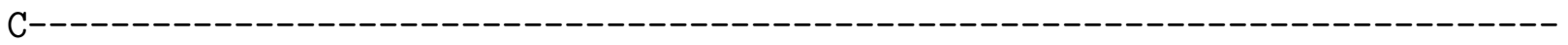

C

C RUN SECTION

$\mathrm{C}$

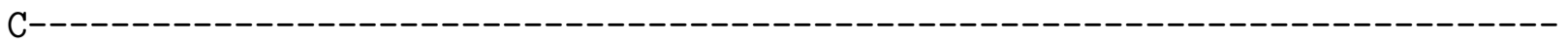

C

C Calculate control surface positions

C

TEMP_CONT $=\operatorname{DBLE}(\mathrm{U}($ CONT_NUM $))$

$\mathrm{U}($ CONT_NUM $)=\operatorname{SNGL}($ TEMP_CONT - DDU $)$

DHTCMD_L $=U(1)$ 


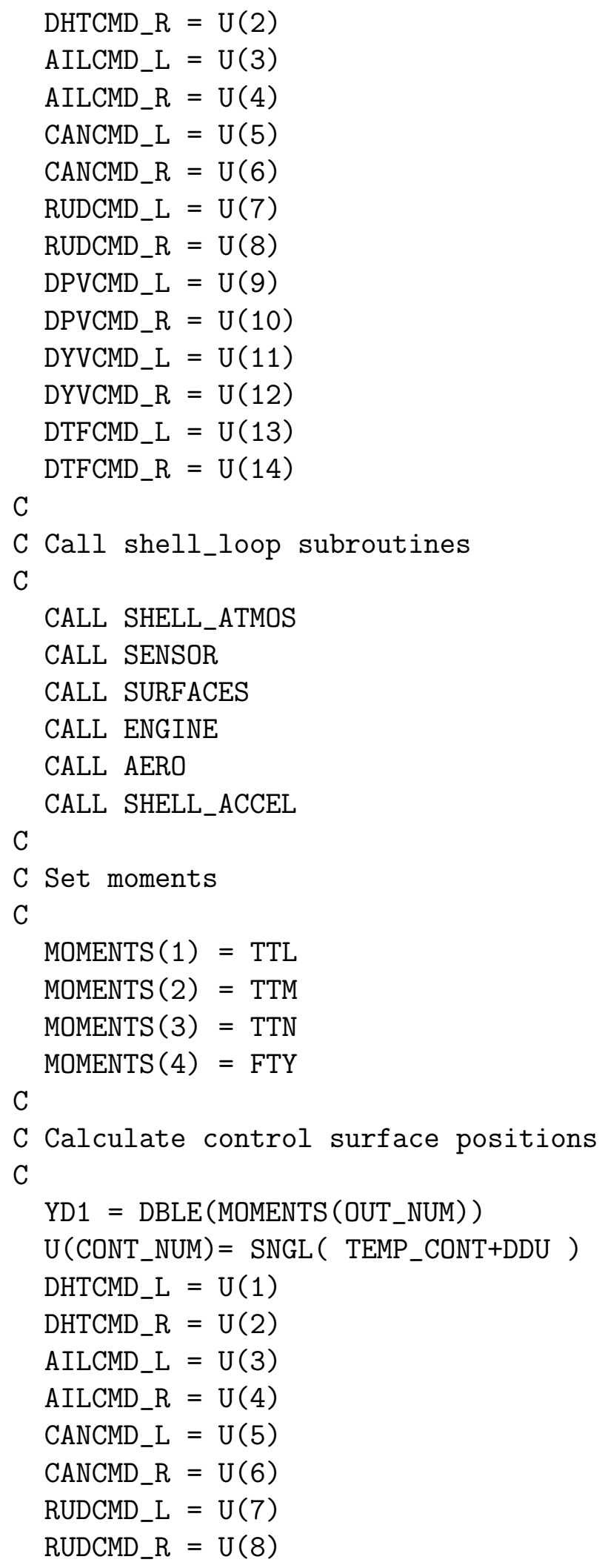




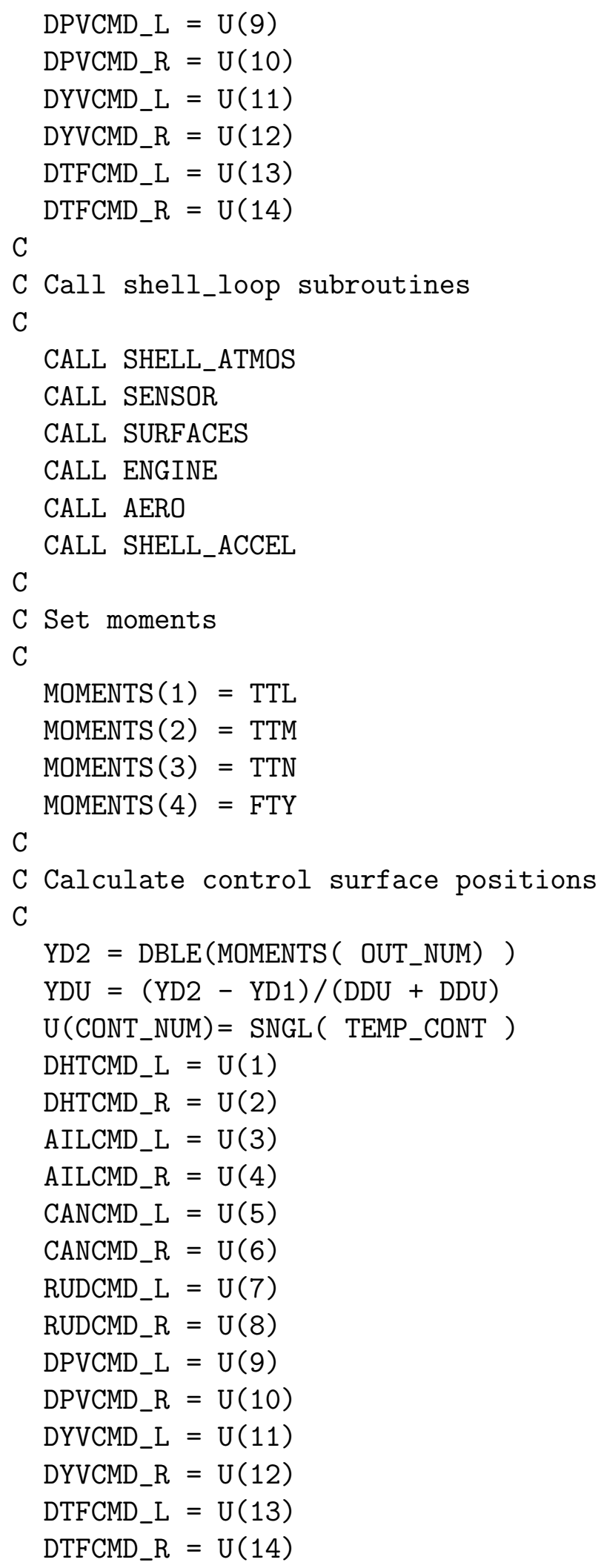




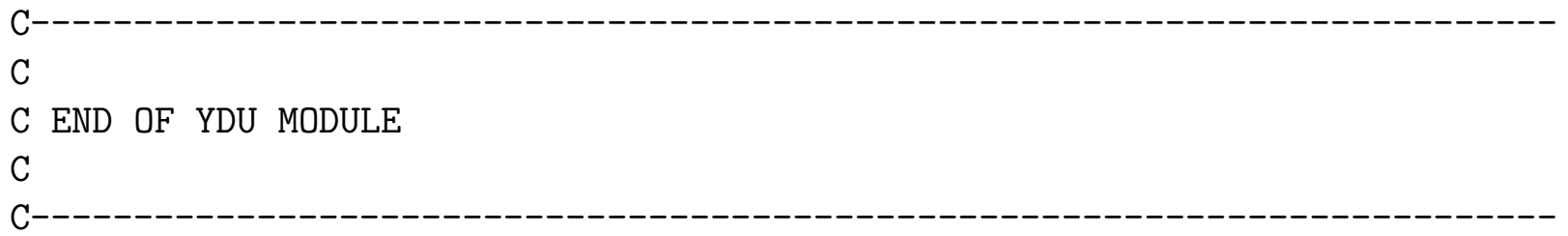

RETURN

END 


\section{Vita}

Kevin Richard Scalera was born on December $7^{\text {th }} 1974$ to Stephen and Patricia Scalera in Providence, Rhode Island. At the age of four he and his family set off for the seacoast town of Hampton, New Hampshire. Kevin quickly acclimated to his new environment and was soon participating in the towns youth sports and recreation programs. However, it was not in athletics that Kevin was meant to leave his mark.

At an early age he established himself as a hardworking and successful student. A reputation for academic excellence, first established by his elder brother, was rapidly growing and developing. His academic career continued to flourish through his high school days where he graduated at the top of his class. From high school Kevin was off to gather further educational enrichment from the Department of Mechanical Engineering at the University of New Hampshire. Kevin's four year stay at UNH demonstrated his honed skills in time management as he orchestrated a masterful balance between academics and social life. He received his Bachelor of Science degree in Mechanical Engineering in the spring of 1997, graduating at the top of his Mechanical Engineering class.

With degree in hand, the next stop along Kevin's great adventure was Virginia Polytechnic Institute and State University. He enrolled in the Masters of Science program in the Department of Aerospace Engineering at Virginia Tech. His first days on campus led him to the Flight Simulation Laboratory. He struggled day by day to convert his Mechanical Engineering mind to that of an Aerospace Engineer. Towards the end of his stay at Virginia Tech, he became the proud uncle and godfather of the beautiful Madison Paige Scalera. But this is a digression. After two years of hard work and determination, intermixed with periods of relaxation and social activity, Kevin completed his research and thesis, albeit extremely verbose. For these accomplishments, during the summer of 1999 he was rewarded with a Masters of Science degree in Aerospace Engineering with a concentration in dynamics and control. It is at this point that the account of Kevin's history ends. The next chapter of the story is about to begin as Kevin prepares to venture out into the real world and once again make his mark. 